Supporting Information

\title{
p-tert-Butyl Groups Improve the Utility of Aromatic Protecting Groups in Carbohydrate Synthesis
}

Sachi Asano ${ }^{b, c}$, Hide-Nori Tanaka ${ }^{a, b}$, Akihiro Imamura ${ }^{b, c}$, Hideharu Ishida ${ }^{a, b, c}$, Hiromune Ando $^{a, b}$

a Center for Highly Advanced Integration of Nano and Life Sciences (G-CHAIN), Gifu University, 1-1 Yanagido, Gifu 501-1193, Japan

${ }^{b}$ The United Graduate School of Agricultural Science, Gifu University, 1-1 Yanagido, Gifu 501-1193, Japan

' Department of Applied Bioorganic Chemistry, Gifu University, 1-1 Yanagido, Gifu 5011193, Japan

Table of contents

1. General information

2. Measurement data for GIcNAc derivatives

3. Synthetic procedures

4. ${ }^{1} \mathrm{H}$ and ${ }^{13} \mathrm{C}$ NMR spectral data for new compounds 


\section{General information}

All reactions were performed under argon atmosphere. All chemicals were purchased from commercial suppliers and used without further purification, if not stated otherwise. Molecular sieves were purchased from Wako Pure Chemical Industries, Ltd. (Osaka, Japan) and pre-dried at $300^{\circ} \mathrm{C}$ for $2 \mathrm{~h}$ in a muffle furnace, and dried in a flask at $300{ }^{\circ} \mathrm{C}$ for $2 \mathrm{~h}$ in vacuo prior to use. Dry solvents for reaction media $\left(\mathrm{CH}_{2} \mathrm{Cl}_{2}\right.$, toluene, $\mathrm{THF}, \mathrm{MeCN}, \mathrm{DMF}$ and pyridine) were purchased from Kanto Chemical Co. Inc. (Tokyo, Japan) and used without purification. Other solvents for reaction media were dried over molecular sieves and used without purification. TLC analysis was performed on Merck TLC plates (silica gel $60 \mathrm{~F}_{254}$ on glass plate), and visualized either by exposure to UV light (253.6 $\mathrm{nm}$ ) or by soak in a solution of $10 \% \mathrm{H}_{2} \mathrm{SO}_{4}$ in ethanol followed by heating. Purification by flash column chromatography was performed on silica gel (80 mesh and 300 mesh; Fuji Silysia Chemical, Ltd. (Aichi, Japan)). The quantity of silica gel was usually estimated as 50 to 100 -fold weight of crude sample to be charged. Solvent systems for chromatography were specified as $v / v$ ratios. Evaporation, concentration and drying-up were carried out in vacuo. ${ }^{1} \mathrm{H}$ NMR and ${ }^{13} \mathrm{C}$ NMR spectra were recorded on $500 \mathrm{MHz}$ spectrometers (Biospin AVANCE III, Bruker, Billerica, MA, USA). Chemical shifts ( $\delta$ ) were measured in ppm relative to TMS $\delta=0.00 \mathrm{ppm}$ as internal standard. Data are presented as follows: chemical shift, multiplicity $(s=$ singlet,$d=$ doublet,$t=$ triplet, $q=$ quartet, quin = quintet, sext = sextet, $\mathrm{dd}=$ double doublet, $\mathrm{dt}=$ double triplet, $\mathrm{m}=$ multiplet and/or multiple resonances), integration, coupling constant in hertz $(\mathrm{Hz})$, and position of the corresponding proton. COSY methods were used to confirm the NMR signal assignments. High-resolution mass (ESI-TOF MS) spectra were taken by Bruker micrOTOF. Optical rotations were measured with a high-sensitivity polarimeter (SEPA-300, Horiba (Kyoto, Japan)). All melting points were measured by melting point apparatus (AS ONE Co. (Osaka, Japan)) and obtained for all crystalline compounds. 


\section{Measurement data for GlcNAc derivatives}

\begin{tabular}{|c|c|c|c|c|}
\hline compd & $\mathrm{mp}\left({ }^{\circ} \mathrm{C}\right)$ & solubility ${ }^{a}(\mathrm{mg} / \mathrm{mL})$ & particle size ${ }^{b}(\mathrm{~nm})$ & label $^{d}$ \\
\hline $10 a$ & $300<$ & 1.0 & $-c$ & $\bullet$ \\
\hline $10 \mathrm{~b}$ & $201-205$ & 22.9 & 0.9 & $\bullet$ \\
\hline $10 c$ & $242-248$ & 6.5 & 357.5 & ○ \\
\hline $10 d$ & $236-240$ & 93.3 & 1.1 & $\bullet$ \\
\hline $11 a$ & $300<$ & 0.5 & $-c$ & $\bullet$ \\
\hline $11 b$ & $243-246$ & 10.6 & 242.0 & - \\
\hline $11 \mathrm{c}$ & $282-285$ & 5.0 & $-c$ & $\bullet$ \\
\hline 11d & $223-226$ & 51.6 & 0.8 & $\bullet$ \\
\hline $12 a$ & $300<$ & 0.9 & $-c$ & - \\
\hline $12 b$ & $232-236$ & 16.5 & 1.4 & $\bullet$ \\
\hline $12 \mathrm{c}$ & $243-247$ & 3.7 & $-c$ & $\bullet$ \\
\hline $12 d$ & $187-193$ & 24.8 & 1.1 & $\bullet$ \\
\hline $13 a$ & $300<$ & 0.6 & $-c$ & $\bullet$ \\
\hline $13 b$ & $240-245$ & 10.9 & 966.9 & $\bullet$ \\
\hline $13 c$ & $290-293$ & 0.9 & $-c$ & - \\
\hline 13d & $231-235$ & 9.5 & 1126.0 & ○ \\
\hline
\end{tabular}

${ }^{a}$ Solubility in EtOH.

${ }^{b} 5 \mathrm{mg} / \mathrm{mL}$ in EtOH. Measured by Zetasizer Nano ZS (Malvern Instruments, Ltd., UK).

${ }^{c}$ Not determined due to low solubility in $\mathrm{EtOH}$.

${ }^{d}$ Each label color corresponds to the plots in Figure 2. 


\section{Synthetic procedures}

\subsection{GalN derivatives}<smiles>COC(OC)c1ccc(C(C)(C)C)cc1</smiles>

\section{1-n-Butyl-4-(dimethoxymethyl)benzene (2b)}

To a solution of compound $\mathbf{1 b}(500 \mathrm{mg}, 3.08 \mathrm{mmol})$ in $\mathrm{MeOH}(6.20 \mathrm{~mL})$ were added trimethyl orthoformate $(1.01 \mathrm{~mL})$ and $\mathrm{HCl}(37 \%, 1.9 \mu \mathrm{L}, 22.1 \mu \mathrm{mol})$. After stirring for $21 \mathrm{~h}$ under reflux as the progress of the reaction was monitored by TLC ( $n$-hexane/EtOAC $=12 / 1$, developed twice), the reaction mixture was cooled to ambient temperature and extracted with EtOAc. The organic layer was washed with $2 \mathrm{M} \mathrm{NaOH}$, dried over $\mathrm{Na}_{2} \mathrm{SO}_{4}$, and concentrated. The residue was purified by silica gel column chromatography $\left(\mathrm{CHCl}_{3}\right)$ to give compound $\mathbf{2 b}$ ( $\left.559 \mathrm{mg}, 87 \%\right)$ as a colorless syrup; ${ }^{1} \mathrm{H}$ NMR $\left(500 \mathrm{MHz}, \mathrm{CDCl}_{3}\right) \delta$ 7.35-7.17 (m, $\left.4 \mathrm{H}, \mathrm{Ar}\right), 5.36(\mathrm{~s}, 1 \mathrm{H}, \mathrm{ArCH}<), 3.33(\mathrm{~s}, 6 \mathrm{H}, 2 \mathrm{OMe}), 2.61(\mathrm{t}, 2 \mathrm{H}$, $\operatorname{ArCH}_{2} \mathrm{CH}_{2} \mathrm{CH}_{2}$ ), 1.62-1.56 (m, $2 \mathrm{H}, \mathrm{ArCH}_{2} \mathrm{CH}_{2} \mathrm{CH}_{2}$ ), 1.35 (sext, $2 \mathrm{H}, \mathrm{ArCH}_{2} \mathrm{CH}_{2} \mathrm{CH}_{2}$ ), $0.92\left(\mathrm{t}, 3 \mathrm{H}, \mathrm{Me}\right.$ ); ${ }^{13} \mathrm{C}$ NMR $\left(125 \mathrm{MHz}, \mathrm{CDCl}_{3}\right) \delta 143.2,135.4,128.3,126.6,103.4,52.8,35.4,33.6,22.3,13.9 ;$ HRMS (ESI) $\mathrm{m} / \mathrm{z}$ : found $[\mathrm{M}+\mathrm{Na}]^{+} 231.1360, \mathrm{C}_{13} \mathrm{H}_{20} \mathrm{O}_{2}$ calcd for $[\mathrm{M}+\mathrm{Na}]^{+} 231.1356$.

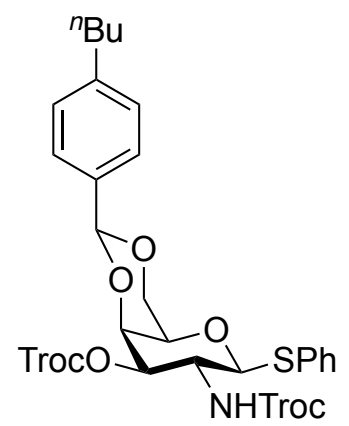

Phenyl 4,6-O-p-n-butylbenzylidene-2-deoxy-1-thio-2-(2,2,2-trichloroethoxycarbam oyl)-3-O-(2,2,2-trichloroethoxycarbonyl)- $\beta$-D-galactopyranoside (4b)

To a solution of compound $3^{501}(295 \mathrm{mg}, 0.660 \mathrm{mmol})$ in $\mathrm{MeCN} / \mathrm{THF}=2 / 1(6.60 \mathrm{~mL})$ were added compound $\mathbf{2 b}(275 \mathrm{mg}, 1.32 \mathrm{mmol})$ and $( \pm)$-10-camphorsulfonic acid $(61.3 \mathrm{mg}, 0.264 \mathrm{mmol})$ at $0{ }^{\circ} \mathrm{C}$. After stirring for $1 \mathrm{~h}$ at ambient temperature as the progress of the reaction was monitored by TLC $\left(\mathrm{CHCl}_{3} / \mathrm{MeOH}=10 / 1\right)$, the reaction mixture was quenched with $\mathrm{Et}_{3} \mathrm{~N}$ and concentrated. The residue was dried for $2 \mathrm{~h}$. Then, the residue was dissolved in pyridine $(6.60 \mathrm{~mL})$, and TrocCl $(566 \mu \mathrm{L}, 4.22$ 
$\mathrm{mmol}$ ) was added at $0{ }^{\circ} \mathrm{C}$. After stirring for $30 \mathrm{~min}$ at ambient temperature as the progress of the reaction was monitored by TLC ( $n$-hexane/EtOAC $=1 / 1)$, the reaction mixture was co-evaporated with toluene. The residue was extracted with $\mathrm{CHCl}_{3}$ and washed with $2 \mathrm{M} \mathrm{HCl}, \mathrm{H}_{2} \mathrm{O}$, satd. aq. $\mathrm{NaHCO}_{3}$ and brine. The organic layer was dried over $\mathrm{Na}_{2} \mathrm{SO}_{4}$ and concentrated. The residue was purified by silica gel column chromatography $\left(\mathrm{CHCl}_{3} / \mathrm{EtOAC}=70 / 1 \rightarrow 5 / 1\right)$ and crystallized ( $n$-hexane/EtOAc) to give compound $4 \mathrm{~b}$ (1.68 g, 82\%) as white crystals; mp $183-188^{\circ} \mathrm{C} ;[\alpha]_{\mathrm{D}}+19.2^{\circ}$ (c $1.0, \mathrm{CHCl}_{3}$ ); ${ }^{1} \mathrm{H}$ NMR (500 $\mathrm{MHz}$, Acetone- $\left.d_{6}\right) \delta 7.65-7.20(\mathrm{~m}, 9 \mathrm{H}, 2 \mathrm{Ar}), 7.17\left(\mathrm{~d}, 1 \mathrm{H}, J_{2, \mathrm{NH}}=9.0 \mathrm{~Hz}, \mathrm{NH}-2\right), 5.64(\mathrm{~s}, 1 \mathrm{H}, \mathrm{ArCH}<)$, 5.24-5.21 (m, $2 \mathrm{H}, \mathrm{H}-1, \mathrm{H}-3), 4.89\left(\mathrm{~s}, 2 \mathrm{H}, 2 \mathrm{C}(\mathrm{O}) \mathrm{OCH}_{2} \mathrm{CCl}_{3}\right), 4.82\left(\mathrm{~d}, 1 \mathrm{H}, \mathrm{J}_{\mathrm{gem}}=12.3 \mathrm{~Hz}, \mathrm{C}(\mathrm{O}) \mathrm{OCH}_{2} \mathrm{CCl}_{3}\right)$, $4.77\left(\mathrm{~d}, 1 \mathrm{H}, \mathrm{C}(\mathrm{O}) \mathrm{OCH}_{2} \mathrm{CCl}_{3}\right), 4.65\left(\mathrm{~d}, 1 \mathrm{H}, J_{3,4}=3.5 \mathrm{~Hz}, \mathrm{H}-4\right), 4.26\left(\mathrm{dd}, 1 \mathrm{H}, J_{5,6 \mathrm{a}}=1.5 \mathrm{~Hz}, J_{\text {gem }}=12.5 \mathrm{~Hz}\right.$, $\mathrm{H}-6 \mathrm{a}), 4.20-4.14(\mathrm{~m}, 2 \mathrm{H}, \mathrm{H}-2, \mathrm{H}-6 \mathrm{~b}), 3.89$ (d, $1 \mathrm{H}, \mathrm{H}-5), 2.63$ (t, $\left.2 \mathrm{H}, \mathrm{ArCH}_{2} \mathrm{CH}_{2} \mathrm{CH}_{2}\right), 1.64-1.58(\mathrm{~m}, 2$ $\mathrm{H}, \mathrm{ArCH}_{2} \mathrm{CH}_{2} \mathrm{CH}_{2}$ ), 1.36 (sext, $2 \mathrm{H}, \mathrm{ArCH}_{2} \mathrm{CH}_{2} \mathrm{CH}_{2}$ ), 0.93 (t, $3 \mathrm{H}, \mathrm{Me}$ ); ${ }^{13} \mathrm{C}$ NMR (125 MHz, Acetone- $d_{6}$ ) $\delta$ 155.0, 154.3, 144.4, 136.8, 133.9, 133.1, 129.7, 128.8, 128.3, 127.3, 101.5, 96.9, 95.6, 86.5, 77.8, 77.4, 74.8, 73.6, 70.4, 69.7, 51.4, 36.0, 34.5, 22.9, 14.2; HRMS (ESI) $\mathrm{m} / \mathrm{z}$ : found $[\mathrm{M}+\mathrm{Na}]^{+}$785.9798, $\mathrm{C}_{29} \mathrm{H}_{31} \mathrm{Cl}_{6} \mathrm{NO}_{8} \mathrm{~S}$ calcd for $[\mathrm{M}+\mathrm{Na}]^{+} 785.9794$.

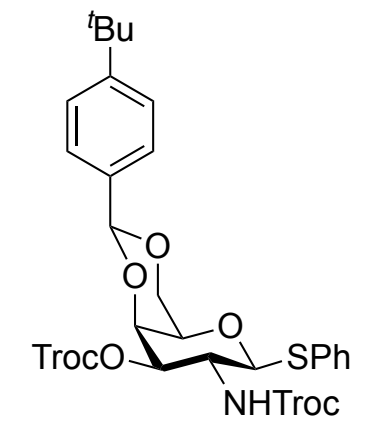

Phenyl 4,6-O-p-tert-butylbenzylidene-2-deoxy-1-thio-2-(2,2,2-trichloroethoxycarbam oyl)-3-O-(2,2,2-trichloroethoxycarbonyl)- $\beta$-D-galactopyranoside (4c)

To a solution of compound $\mathbf{3}^{\mathrm{s} 01}(201 \mathrm{mg}, 0.450 \mathrm{mmol})$ in $\mathrm{MeCN} / \mathrm{THF}=2 / 1(4.50 \mathrm{~mL})$ were added compound $2 \mathrm{c}$ (187 mg, $0.900 \mathrm{mmol}$ ) and ( \pm --10-camphorsulfonic acid $\left(41.8 \mathrm{mg}, 0.180 \mathrm{mmol}\right.$ ) at $0{ }^{\circ} \mathrm{C}$. After stirring for $2.5 \mathrm{~h}$ at ambient temperature as the progress of the reaction was monitored by TLC $\left(\mathrm{CHCl}_{3} / \mathrm{MeOH}=10 / 1\right)$, the reaction mixture was quenched with $\mathrm{Et}_{3} \mathrm{~N}$ and concentrated. The residue was dried for $2 \mathrm{~h}$. Then, the residue was dissolved in pyridine $(4.50 \mathrm{~mL})$, and TrocCl (181 $\mu \mathrm{L}, 1.35$ $\mathrm{mmol}$ ) was added at $0{ }^{\circ} \mathrm{C}$. After stirring for $1 \mathrm{~h}$ at ambient temperature as the progress of the reaction was monitored by TLC ( $n$-hexane/EtOAC $=1 / 1)$, the reaction mixture was co-evaporated with toluene. The residue was extracted with $\mathrm{CHCl}_{3}$ and washed with $2 \mathrm{M} \mathrm{HCl}, \mathrm{H}_{2} \mathrm{O}$, satd. aq. $\mathrm{NaHCO}_{3}$ and brine. The organic layer was dried over $\mathrm{Na}_{2} \mathrm{SO}_{4}$ and concentrated. The residue was purified by silica gel 
column chromatography ( $n$-hexane $/ \mathrm{CHCl}_{3} / \mathrm{EtOAc}=5 / 1 / 1$ ) and crystallized ( $n$-hexane/EtOAc) to give compound 4c (310 mg, 90\%) as white crystals; mp 141-147 ${ }^{\circ} \mathrm{C}$; $[\alpha]_{\mathrm{D}}+17.5^{\circ}$ (c 1.0, $\mathrm{CHCl}_{3}$ ); ${ }^{1} \mathrm{H} \mathrm{NMR}$ (500 MHz, Acetone- $\left.d_{6}\right) \delta$ 7.64-7.29 (m, $9 \mathrm{H}, 2 \mathrm{Ar}$ ), $7.17\left(\mathrm{~d}, 1 \mathrm{H}, J_{2, \mathrm{NH}}=9.0 \mathrm{~Hz}, \mathrm{NH}-2\right), 5.65(\mathrm{~s}, 1 \mathrm{H}$, $\operatorname{ArCH}<), 5.24-5.21(\mathrm{~m}, 2 \mathrm{H}, \mathrm{H}-1, \mathrm{H}-3), 4.89\left(\mathrm{~s}, 2 \mathrm{H}, 2 \mathrm{C}(\mathrm{O}) \mathrm{OCH}_{2} \mathrm{CCl}_{3}\right), 4.82\left(\mathrm{~d}, 1 \mathrm{H}, J_{\mathrm{gem}}=12.5 \mathrm{~Hz}\right.$, $\left.\mathrm{C}(\mathrm{O}) \mathrm{OCH}_{2} \mathrm{CCl}_{3}\right), 4.77\left(\mathrm{~d}, 1 \mathrm{H}, \mathrm{C}(\mathrm{O}) \mathrm{OCH}_{2} \mathrm{CCl}_{3}\right), 4.65\left(\mathrm{~d}, 1 \mathrm{H}, \mathrm{J}_{3,4}=3.5 \mathrm{~Hz}, \mathrm{H}-4\right), 4.27\left(\mathrm{dd}, 1 \mathrm{H}, \mathrm{J}_{5,6 \mathrm{a}}=1.5\right.$ $\left.\mathrm{Hz}, J_{\text {gem }}=12.5 \mathrm{~Hz}, \mathrm{H}-6 \mathrm{a}\right), 4.21-4.15(\mathrm{~m}, 2 \mathrm{H}, \mathrm{H}-2, \mathrm{H}-6 \mathrm{~b}), 3.89(\mathrm{~d}, 1 \mathrm{H}, \mathrm{H}-5), 1.33\left(\mathrm{~s}, 9 \mathrm{H},{ }^{\mathrm{t}} \mathrm{Bu}\right) ;{ }^{13} \mathrm{C} \mathrm{NMR}$ (125 MHz, Acetone- $\left.d_{6}\right) \delta$ 155.1, 154.3, 152.5, 136.6, 134.0, 133.0, 129.7, 128.3, 127.1, 125.6, 101.4, 96.9, 95.6, 86.6, 79.2, 77.8, 77.4, 74.8, 73.6, 70.4, 69.7, 51.4, 35.2, 31.6; HRMS (ESI) $m / z$ : found $[\mathrm{M}+\mathrm{Na}]^{+} 785.9796, \mathrm{C}_{29} \mathrm{H}_{31} \mathrm{Cl}_{6} \mathrm{NO}_{8} \mathrm{~S}$ calcd for $[\mathrm{M}+\mathrm{Na}]^{+} 785.9794$.

\subsection{GaIN + Trisaccharide}

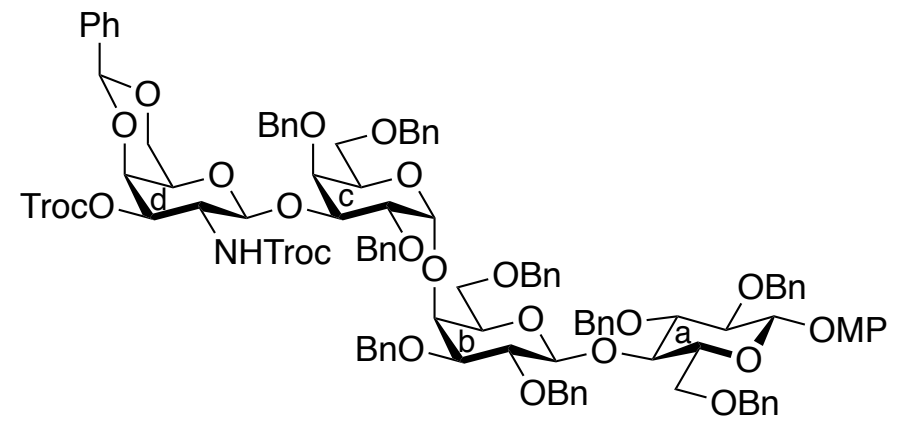

p-Methoxyphenyl

[4,6-O-benzylidene-2-deoxy-1-thio-2-(2,2,2-

trichloroethoxycarbamoyl)-3-O-(2,2,2-trichloroethoxycarbonyl)- $\beta$-D-

galactopyranosyl]-(1 $\rightarrow 3)-(2,4,6$-tri-O-benzyl- $\alpha$-D-galactopyranosyl)-(1 $\rightarrow$ 4)-(2,3,6-tri$O$-benzyl- $\beta$-D-galactopyranosyl)-(1 $\rightarrow 4)$-2,3,6-tri-O-benzyl- $\beta$-D-glucopyranoside (6a)

To a solution of donor $4 \mathrm{a}(93.8 \mathrm{mg}, 0.132 \mathrm{mmol})$ and acceptor 5 (188 $\mathrm{mg}, 0.132 \mathrm{mmol})$ in $\mathrm{CH}_{2} \mathrm{Cl}_{2}(1.30$ $\mathrm{mL}$ ) were added NIS (59.2 mg, $0.264 \mathrm{mmol}$ ) and 4 Å molecular sieves (300 mg). After stirring for $1 \mathrm{~h}$ at ambient temperature, $\mathrm{TfOH}(2.3 \mu \mathrm{L}, 26.4 \mu \mathrm{mol})$ was added at $-80^{\circ} \mathrm{C}$. After stirring for $1 \mathrm{~h}$ at $-80^{\circ} \mathrm{C}$ as the progress of the reaction was monitored by TLC ( $n$-hexane $/ \mathrm{CHCl}_{3} /$ acetone $\left.=3 / 1 / 1\right)$, the reaction mixture was neutralized with satd. aq. $\mathrm{NaHCO}_{3}$ and filtered through a pad of Celite. The pad was washed with $\mathrm{CHCl}_{3}$. The combined filtrate and washings were diluted with $\mathrm{CHCl}_{3}$, and washed with satd. aq. $\mathrm{Na}_{2} \mathrm{~S}_{2} \mathrm{O}_{3}$ and brine. The organic layer was dried over $\mathrm{Na}_{2} \mathrm{SO}_{4}$ and concentrated. The residue was purified by silica gel column chromatography ( $n$-hexane/ $\mathrm{CHCl}_{3} /$ acetone $=6 / 1 / 1$ ) to give compound 6a (228 mg, 85\%) as a white foam; $[\alpha]_{D}+12.8^{\circ}$ (c 1.0, $\left.\mathrm{CHCl}_{3}\right) ;{ }^{1} \mathrm{H} \mathrm{NMR} \mathrm{(500} \mathrm{MHz,} \mathrm{Acetone-}$ $\left.d_{6}\right) \delta 7.58-6.80(\mathrm{~m}, 54 \mathrm{H}, 11 \mathrm{Ar}), 6.53\left(\mathrm{~d}, 1 \mathrm{H}, \mathrm{J}_{2, \mathrm{NH}}=9.5 \mathrm{~Hz}, \mathrm{NH}-2^{d}\right), 5.60(\mathrm{~s}, 1 \mathrm{H}, \mathrm{PhCH}<), 5.31(\mathrm{~d}, 1 \mathrm{H}$, 
$\left.J_{\text {gem }}=11.0 \mathrm{~Hz}, \mathrm{PhCH}_{2}\right), 5.19\left(\mathrm{~d}, 1 \mathrm{H}, J_{\text {gem }}=11.5 \mathrm{~Hz}, \mathrm{PhCH} 2\right), 5.01\left(\mathrm{~d}, 1 \mathrm{H}, J_{1,2}=3.3 \mathrm{~Hz}, \mathrm{H}-1^{c}\right), 4.97(\mathrm{~d}, 1$ $\left.\mathrm{H}, J_{1,2}=7.5 \mathrm{~Hz}, \mathrm{H}-1^{d}\right), 4.97-4.79\left(\mathrm{~m}, 11 \mathrm{H}, \mathrm{H}-1^{d}, \mathrm{H}-3^{d}, 7 \mathrm{PhCH}_{2}, 2 \mathrm{C}(\mathrm{O}) \mathrm{OCH}_{2} \mathrm{CCl}_{3}\right), 4.75\left(\mathrm{~d}, 1 \mathrm{H}, J_{\text {gem }}=\right.$ $\left.12.5 \mathrm{~Hz}, \mathrm{PhCH}_{2}\right), 4.66\left(\mathrm{~d}, 1 \mathrm{H}, \mathrm{J}_{1,2}=7.8 \mathrm{~Hz}, \mathrm{H}-1^{b}\right), 4.64-4.57\left(\mathrm{~m}, 3 \mathrm{H}, 2 \mathrm{PhCH}_{2}, \mathrm{C}(\mathrm{O}) \mathrm{OCH}_{2} \mathrm{CCl}_{3}\right), 4.51-$ $4.49\left(\mathrm{~m}, 2 \mathrm{H}, \mathrm{H}-5^{c}, \mathrm{PhCH}_{2}\right), 4.44\left(\mathrm{~d}, 1 \mathrm{H}, \mathrm{J}_{3,4}=3.5 \mathrm{~Hz}, \mathrm{H}-4^{d}\right), 4.42-4.31\left(\mathrm{~m}, 6 \mathrm{H}, \mathrm{H}-3^{c}, \mathrm{H}-4^{c}, 3 \mathrm{PhCH}_{2}\right.$, $\left.\mathrm{C}(0) \mathrm{OCH}_{2} \mathrm{CCl}_{3}\right), 4.29-4.17\left(\mathrm{~m}, 5 \mathrm{H}, \mathrm{H}-4^{b}, \mathrm{H}-2^{d}, \mathrm{H}-6^{d}, 2 \mathrm{PhCH}_{2}\right), 4.08-4.04\left(\mathrm{~m}, 3 \mathrm{H}, \mathrm{H}-4^{a}, \mathrm{H}-6^{b}, \mathrm{H}-6^{\prime} d\right)$, $3.99\left(\mathrm{dd}, 1 \mathrm{H}, J_{2,3}=10.0 \mathrm{~Hz}, \mathrm{H}-2^{c}\right.$ ), $3.88\left(\mathrm{dd}, 1 \mathrm{H}, J_{5,6}=4.3 \mathrm{~Hz}, J_{\text {gem }}=10.8 \mathrm{~Hz}, \mathrm{H}-6^{a}\right), 3.84\left(\mathrm{dd}, 1 \mathrm{H}, J_{5,6^{\prime}}\right.$ $\left.=1.8 \mathrm{~Hz}, \mathrm{H}-6^{\prime a}\right), 3.79\left(\mathrm{t}, 1 \mathrm{H}, \mathrm{J}_{2,3}=7.5 \mathrm{~Hz}, \mathrm{H}-2^{b}\right), 3.77-3.67\left(\mathrm{~m}, 6 \mathrm{H}, \mathrm{H}-3^{a}, \mathrm{H}-5^{a}, \mathrm{H}-6^{\prime b}, \mathrm{OMe}\right), 3.60-3.51$ $\left(\mathrm{m}, 4 \mathrm{H}, \mathrm{H}-2^{a}, \mathrm{H}-3^{b}, \mathrm{H}-5^{b}, \mathrm{H}-6^{c}\right), 3.28\left(\mathrm{dd}, 1 \mathrm{H}, J_{5,6^{\prime}}=5.0 \mathrm{~Hz}, J_{\mathrm{gem}}=8.5 \mathrm{~Hz}, \mathrm{H}-6^{\prime c}\right), 3.02\left(\mathrm{~s}, 1 \mathrm{H}, \mathrm{H}-5^{d}\right) ;{ }^{13} \mathrm{C}$ NMR (125 MHz, Acetone- $\left.d_{6}\right) \delta$ 156.1, 155.1, 154.2, 152.6, 140.9, 140.6, 140.1, 140.0, 140.0, 140.0, 139.7, 139.6, 139.3, 130.3, 129.6, 129.2, 129.1, 129.0, 129.0, 129.0, 128.8, 128.7, 128.7, 128.7, 128.4, $128.4,128.3,128.3,128.2,128.2,128.1,128.0,127.8,127.4,118.8,115.3,103.5,103.1,102.9,101.6$, 101.4, 96.7, 95.6, 82.5, 82.1, 81.1, 80.4, 79.0, 78.7, 77.4, 77.3, 76.6, 76.5, 76.0, 75.9, 75.7, 75.7, 75.4, 75.0, 74.8, 74.7, 74.4, 73.6, 73.4, 73.1, 72.6, 70.0, 69.7, 69.5, 69.3, 68.9, 66.3, 55.8, 53.4; HRMS (ESI) $\mathrm{m} / \mathrm{z}$ : found $[\mathrm{M}+\mathrm{Na}]^{+} 2040.5310, \mathrm{C}_{107} \mathrm{H}_{109} \mathrm{Cl}_{6} \mathrm{NO}_{25}$ calcd for $[\mathrm{M}+\mathrm{Na}]^{+} 2040.5312$.

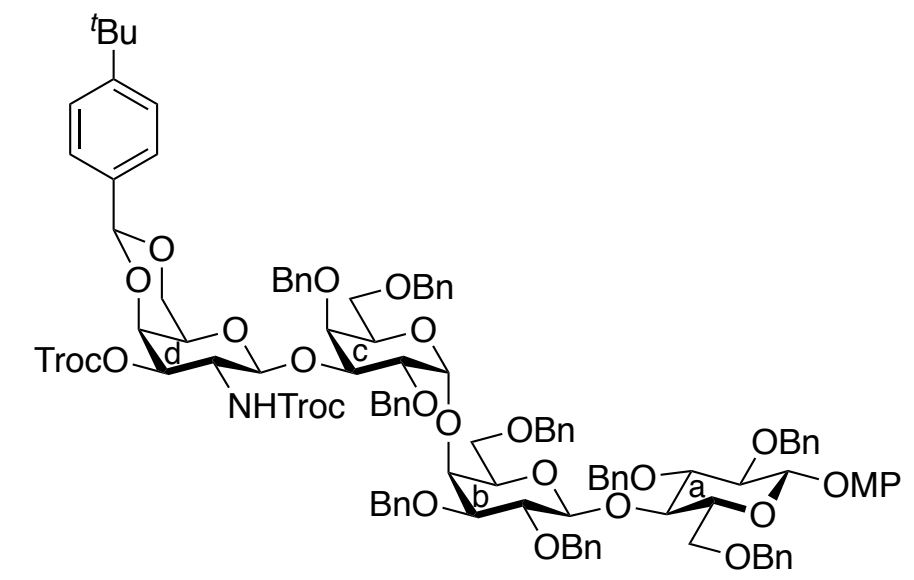

p-Methoxyphenyl

[4,6-O-p-tert-butylbenzylidene-2-deoxy-1-thio-2-(2,2,2trichloroethoxycarbamoyl)-3-O-(2,2,2-trichloroethoxycarbonyl)- $\beta$-Dgalactopyranosyl]-(1 $\rightarrow 3)-(2,4,6$-tri-O-benzyl- $\alpha$-D-galactopyranosyl)-(1 $\rightarrow 4)-(2,3,6$-tri-Obenzyl- $\beta$-D-galactopyranosyl)-(1 $\rightarrow 4)-2,3,6$-tri-O-benzyl- $\beta$-D-glucopyranoside (6c)

To a solution of donor $4 \mathrm{c}(177 \mathrm{mg}, 0.124 \mathrm{mmol})$ and acceptor $5(95.0 \mathrm{mg}, 0.124 \mathrm{mmol})$ in $\mathrm{CH}_{2} \mathrm{Cl}_{2}(1.20$ $\mathrm{mL}$ ) were added NIS ( $55.6 \mathrm{mg}, 0.248 \mathrm{mmol}$ ) and 4 Å molecular sieves ( $300 \mathrm{mg}$ ). After stirring for $1 \mathrm{~h}$ at ambient temperature, $\mathrm{TfOH}(2.2 \mu \mathrm{L}, 24.8 \mu \mathrm{mol})$ was added at $-80^{\circ} \mathrm{C}$. After stirring for $10 \mathrm{~min}$ at $-80^{\circ} \mathrm{C}$ as the progress of the reaction was monitored by TLC ( $n$-hexane $/ \mathrm{CHCl}_{3} /$ acetone $\left.=3 / 1 / 1\right)$, the reaction mixture was neutralized with satd. aq. $\mathrm{NaHCO}_{3}$ and filtered through a pad of Celite. The pad 
was washed with $\mathrm{CHCl}_{3}$. The combined filtrate and washings were diluted with $\mathrm{CHCl}_{3}$, and washed with satd. aq. $\mathrm{Na}_{2} \mathrm{~S}_{2} \mathrm{O}_{3}$ and brine. The organic layer was dried over $\mathrm{Na}_{2} \mathrm{SO}_{4}$ and concentrated. The residue was purified by silica gel column chromatography $\left(1^{\text {st: }} n\right.$-hexane $/ \mathrm{CHCl}_{3} /$ acetone $=6 / 1 / 1,2^{\text {ndd }}$ : $n$-hexane $/ \mathrm{CHCl}_{3} /$ acetone $\left.=7 / 1 / 1\right)$ to give compound $6 \mathrm{c}(227 \mathrm{mg}, 88 \%)$ as a white foam; $[\alpha]_{\mathrm{D}}+13.7^{\circ}$ (c 1.0, $\left.\mathrm{CHCl}_{3}\right) ;{ }^{1} \mathrm{H}$ NMR (500 MHz, Acetone- $\left.d_{6}\right) \delta 7.58-6.80(\mathrm{~m}, 53 \mathrm{H}, 11 \mathrm{Ar}), 6.52\left(\mathrm{~d}, 1 \mathrm{H}, J_{2, \mathrm{NH}}=9.5\right.$ $\left.\mathrm{Hz}, \mathrm{NH}-2^{d}\right), 5.58(\mathrm{~s}, 1 \mathrm{H}, \mathrm{ArCH}<), 5.35\left(\mathrm{~d}, 1 \mathrm{H}, \mathrm{J}_{\mathrm{gem}}=11.0 \mathrm{~Hz}, \mathrm{PhCH} 2\right), 5.19\left(\mathrm{~d}, 1 \mathrm{H}, J_{\text {gem }}=11.0 \mathrm{~Hz}, \mathrm{PhCH}_{2}\right)$, $5.00\left(\mathrm{~d}, 1 \mathrm{H}, \mathrm{J}_{1,2}=3.5 \mathrm{~Hz}, \mathrm{H}-1^{c}\right), 4.97-4.79\left(\mathrm{~m}, 12 \mathrm{H}, \mathrm{H}-1^{a}, \mathrm{H}-1^{d}, \mathrm{H}-3^{d}, 7 \mathrm{PhCH}_{2}, 2 \mathrm{C}(\mathrm{O}) \mathrm{OCH}_{2} \mathrm{CCl}_{3}\right), 4.75$ $\left(\mathrm{d}, 1 \mathrm{H}, J_{\text {gem }}=12.5 \mathrm{~Hz}, \mathrm{PhCH}_{2}\right), 4.66\left(\mathrm{~d}, 1 \mathrm{H}, J_{1,2}=7.5 \mathrm{~Hz}, \mathrm{H}-1^{b}\right), 4.64\left(\mathrm{~d}, 1 \mathrm{H}, J_{\text {gem }}=12.0 \mathrm{~Hz}\right.$, $\left.\mathrm{C}(\mathrm{O}) \mathrm{OCH}_{2} \mathrm{CCl}_{3}\right), 4.61\left(\mathrm{~d}, 1 \mathrm{H}, J_{\text {gem }}=12.5 \mathrm{~Hz}, \mathrm{PhCH}_{2}\right), 4.59\left(\mathrm{~d}, 1 \mathrm{H}, \mathrm{PhCH}_{2}\right), 4.52-4.48\left(\mathrm{~m}, 2 \mathrm{H}, \mathrm{H}-5^{c}\right.$, $\left.\mathrm{PhCH}_{2}\right), 4.42-4.31\left(\mathrm{~m}, 12 \mathrm{H}, \mathrm{H}-4^{b}, \mathrm{H}-3^{c}, \mathrm{H}-4^{c}, \mathrm{H}-2^{d}, \mathrm{H}-4^{d}, \mathrm{H}-6^{d}, 5 \mathrm{PhCH}_{2}, \mathrm{C}(\mathrm{O}) \mathrm{OCH}_{2} \mathrm{CCl}_{3}\right), 4.08-4.02(\mathrm{~m}$, $\left.3 \mathrm{H}, \mathrm{H}-4^{a}, \mathrm{H}-6^{b}, \mathrm{H}-6^{\prime d}\right), 3.99\left(\mathrm{dd}, 1 \mathrm{H}, J_{2,3}=10.0 \mathrm{~Hz}, \mathrm{H}-2^{c}\right.$ ), $3.88\left(\mathrm{dd}, 1 \mathrm{H}, J_{5,6}=4.5 \mathrm{~Hz}, J_{\mathrm{gem}}=11.0 \mathrm{~Hz}, \mathrm{H}-\right.$ $\left.6^{a}\right), 3.84\left(\mathrm{dd}, 1 \mathrm{H}, \mathrm{J}_{5,6^{\prime}}=1.5 \mathrm{~Hz}, \mathrm{H}-6^{\prime a}\right), 3.81-3.67\left(\mathrm{~m}, 7 \mathrm{H}, \mathrm{H}-3^{a}, \mathrm{H}-5^{a}, \mathrm{H}-2^{b}, \mathrm{H}-6^{\prime b}, \mathrm{OMe}\right), 3.60-3.51(\mathrm{~m}$, $\left.4 \mathrm{H}, \mathrm{H}-2^{a}, \mathrm{H}-3^{b}, \mathrm{H}-5^{b}, \mathrm{H}-6^{c}\right), 3.28\left(\mathrm{dd}, 1 \mathrm{H}, J_{5,6^{\prime}}=4.8 \mathrm{~Hz}, \mathrm{~J}_{\mathrm{gem}}=8.3 \mathrm{~Hz}, \mathrm{H}-6^{\prime c}\right), 3.04\left(\mathrm{~s}, 1 \mathrm{H}, \mathrm{H}-5^{d}\right), 1.22(\mathrm{~s}$, $\left.9 \mathrm{H},{ }^{\mathrm{t}} \mathrm{Bu}\right) ;{ }^{13} \mathrm{C}$ NMR (125 MHz, Acetone- $\left.d_{6}\right) \delta 156.1,155.1,154.3,152.6,152.4,141.0,140.6,140.2$, $140.0,140.0,140.0,139.7,139.6,139.3,136.5,130.3,129.8,129.4,129.2,129.0,129.0,129.0,129.0$, 129.0, 128.9, 128.8, 128.7, 128.7, 128.5, 128.4, 128.3, 128.3, 128.3, 128.2, 128.1, 128.0, 127.8, 127.1, $126.1,125.5,118.8,115.3,103.5,103.2,102.9,101.5,96.7,95.6,82.5,82.1,81.3,80.4,78.8,77.4$, $77.3,76.6,76.5,76.1,76.0,75.7,75.7,75.4,75.0,74.8,74.6,74.3,73.6,73.5,73.4,73.0,72.6,70.0$, 69.7, 69.5, 69.3, 68.9, 66.3, 55.8, 53.4, 35.0, 31.5; HRMS (ESI) $\mathrm{m} / \mathrm{z}$ : found $[\mathrm{M}+\mathrm{Na}]^{+}$2096.5935, $\mathrm{C}_{111} \mathrm{H}_{117} \mathrm{Cl}_{6} \mathrm{NO}_{25}$ calcd for $[\mathrm{M}+\mathrm{Na}]^{+} 2096.5938$.

\subsection{Manipulation of TBBzld group}

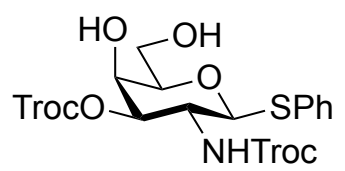

Phenyl 2-deoxy-1-thio-2-(2,2,2-trichloroethoxycarbamoyl)-3-0-(2,2,2-trichloroethoxy carbonyl)- $\beta$-D-galactopyranoside (7)

Compound 4c (20.0 mg, $26.1 \mu \mathrm{mol})$ was dissolved in $\mathrm{AcOH} / \mathrm{H}_{2} \mathrm{O}=4 / 1(0.60 \mathrm{~mL})$. After stirring for $1 \mathrm{~h}$ at $60^{\circ} \mathrm{C}$ as the progress of the reaction was monitored by $\operatorname{TLC}(n$-hexane/EtOAC $=2 / 1)$, the reaction mixture was extracted with $\mathrm{CHCl}_{3}$ and washed with satd. aq. $\mathrm{NaHCO}_{3}$ and brine. The organic layer was dried over $\mathrm{Na}_{2} \mathrm{SO}_{4}$ and concentrated. The residue was purified by silica gel column chromatography ( $n$-hexane/acetone $=4 / 1 \rightarrow 1 / 1)$ to give compound $7(15.9 \mathrm{mg}, 98 \%$ ) as a white 
amorphous solid; $[\alpha]_{D}+3.3^{\circ}$ (c 1.6, $\left.\mathrm{CHCl}_{3}\right) ;{ }^{1} \mathrm{H}$ NMR $\left(500 \mathrm{MHz}\right.$, Acetone- $\left.d_{6}\right) \delta 7.56-7.25(\mathrm{~m}, 5 \mathrm{H}, \mathrm{Ar})$, $7.14\left(\mathrm{~d}, 1 \mathrm{H}, J_{2, \mathrm{NH}}=9.5 \mathrm{~Hz}, \mathrm{NH}-2\right), 5.11\left(\mathrm{~d}, 1 \mathrm{H}, J_{1,2}=10.5 \mathrm{~Hz}, \mathrm{H}-1\right), 5.03\left(\mathrm{dd}, 1 \mathrm{H}, J_{3,4}=2.9 \mathrm{~Hz}, J_{2,3}=\right.$ $10.5 \mathrm{~Hz}, \mathrm{H}-3), 4.92\left(\mathrm{~d}, 1 \mathrm{H}, J_{\mathrm{gem}}=12.0 \mathrm{~Hz}, \mathrm{C}(\mathrm{O}) \mathrm{OCH}_{2} \mathrm{CCl}_{3}\right), 4.87\left(\mathrm{~d}, 1 \mathrm{H}, \mathrm{C}(\mathrm{O}) \mathrm{OCH}_{2} \mathrm{CCl}_{3}\right), 4.83(\mathrm{~d}, 1 \mathrm{H}$, $\left.J_{\text {gem }}=12.0 \mathrm{~Hz}, \mathrm{C}(\mathrm{O}) \mathrm{OCH}_{2} \mathrm{CCl}_{3}\right), 4.79\left(\mathrm{~d}, 1 \mathrm{H}, \mathrm{C}(\mathrm{O}) \mathrm{OCH}_{2} \mathrm{CCl}_{3}\right), 4.65\left(\mathrm{~d}, 1 \mathrm{H}, J_{4, \mathrm{OH}}=5.2 \mathrm{~Hz}, \mathrm{OH}-4\right), 4.35$ (dd, $1 \mathrm{H}, \mathrm{H}-4), 4.23$ (q, $1 \mathrm{H}, \mathrm{H}-2$ ), $4.00\left(\mathrm{t}, 1 \mathrm{H}, \mathrm{J}_{6, \mathrm{OH}}=6.0 \mathrm{~Hz}, \mathrm{OH}-6\right), 3.83-3.80(\mathrm{~m}, 2 \mathrm{H}, \mathrm{H}-6 \mathrm{a}, \mathrm{H}-6 \mathrm{~b}), 3.74$ (t, $\left.1 \mathrm{H}, J_{5,6 \mathrm{a}}=J_{5,6 \mathrm{~b}}=6.0 \mathrm{~Hz}, \mathrm{H}-5\right) ;{ }^{13} \mathrm{C}$ NMR $\left(125 \mathrm{MHz}\right.$, Acetone- $\left.d_{6}\right) \delta$ 155.2, 154.2, 135.6, 131.7, 129.7, $127.9,97.0,95.6,87.9,80.4,79.7,77.5,74.8,66.7,62.0,51.8$; HRMS (ESI) $\mathrm{m} / \mathrm{z}$ : found $[\mathrm{M}+\mathrm{Na}]^{+}$ $641.8855, \mathrm{C}_{18} \mathrm{H}_{19} \mathrm{Cl}_{6} \mathrm{NO}_{8} \mathrm{~S}$ calcd for $[\mathrm{M}+\mathrm{Na}]^{+} 641.8855$.

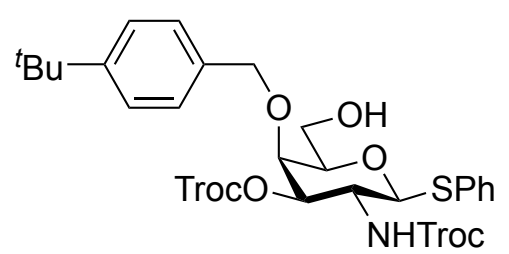

Phenyl 4-O-p-tert-butylbenzyl-2-deoxy-1-thio-2-(2,2,2-trichloroethoxycarbamoyl)-30 -(2,2,2-trichloroethoxycarbonyl)- $\beta$-D-galactopyranoside (8)

To a solution of compound $4 \mathrm{c}(46.1 \mathrm{mg}, 60.2 \mu \mathrm{mol})$ in $\mathrm{CH}_{2} \mathrm{Cl}_{2}(0.60 \mathrm{~mL})$ was added $4 \AA$ molecular sieves $(46.1 \mathrm{mg})$. After stirring for $30 \mathrm{~min}$ at ambient temperature, the reaction mixture was cooled to $-78{ }^{\circ} \mathrm{C}$ and added $\mathrm{Et}_{3} \mathrm{SiH}(21.0 \mu \mathrm{L}, 132 \mu \mathrm{mol})$ and $\mathrm{PhBCl}_{2}(17.5 \mu \mathrm{L}, 132 \mu \mathrm{mol})$. After stirring for 15 $\min$ as the progress of the reaction was monitored by TLC $(n$-hexane/EtOAC $=1 / 1)$, the reaction was quenched with $\mathrm{Et}_{3} \mathrm{~N}$ and $\mathrm{MeOH}$. The reaction mixture was extracted with $\mathrm{CHCl}_{3}$ and washed with satd. aq. $\mathrm{NaHCO}_{3}$ and brine. The organic layer was dried over $\mathrm{Na}_{2} \mathrm{SO}_{4}$ and concentrated. The residue was purified by silica gel column chromatography $(n$-hexane/EtOAC $=1 / 1)$ to give compound $8(45.9 \mathrm{mg}$, $99 \%)$ as a white amorphous solid; $[\alpha]_{\mathrm{D}}+2.6^{\circ}\left(\mathrm{c} 1.1, \mathrm{CHCl}_{3}\right) ;{ }^{1} \mathrm{H}$ NMR $\left(500 \mathrm{MHz}\right.$, Acetone- $\left.d_{6}\right) \delta 7.53-$ $7.23\left(\mathrm{~m}, 9 \mathrm{H}, 2 \mathrm{Ar}\right.$ ), 7.17 (d, $\left.1 \mathrm{H}, J_{2, \mathrm{NH}}=9.5 \mathrm{~Hz}, \mathrm{NH}-2\right), 5.18$ (dd, $1 \mathrm{H}, J_{3,4}=3.0 \mathrm{~Hz}, J_{2,3}=10.5 \mathrm{~Hz}, \mathrm{H}-3$ ), $5.11\left(\mathrm{~d}, 1 \mathrm{H}, \mathrm{J}_{1,2}=10.0 \mathrm{~Hz}, \mathrm{H}-1\right), 5.02\left(\mathrm{~d}, 1 \mathrm{H}, \mathrm{J}_{\mathrm{gem}}=12.0 \mathrm{~Hz}, \mathrm{C}(\mathrm{O}) \mathrm{OCH}_{2} \mathrm{CCl}_{3}\right), 4.89$ (d, $1 \mathrm{H}, \mathrm{C}(\mathrm{O}) \mathrm{OCH}_{2} \mathrm{CCl}_{3}$ ), $4.84\left(\mathrm{~d}, 1 \mathrm{H}, \mathrm{C}(\mathrm{O}) \mathrm{OCH}_{2} \mathrm{CCl}_{3}\right), 4.79-4.77\left(\mathrm{~m}, 2 \mathrm{H}, \mathrm{C}(\mathrm{O}) \mathrm{OCH}_{2} \mathrm{CCl}_{3}, \mathrm{ArCH}_{2}\right), 4.66\left(\mathrm{~d}, 1 \mathrm{H}, J_{\text {gem }}=10.5 \mathrm{~Hz}\right.$, $\operatorname{ArCH}_{2}$ ), 4.28 (d, $\left.1 \mathrm{H}, \mathrm{H}-4\right), 4.21$ (q, $\left.1 \mathrm{H}, \mathrm{H}-2\right), 4.00$ (dd, $\left.1 \mathrm{H}, \mathrm{OH}-6\right), 3.83-3.75$ (m, $3 \mathrm{H}, \mathrm{H}-5, \mathrm{H}-6 \mathrm{a}, \mathrm{H}-6 \mathrm{~b}$ ), $1.33\left(\mathrm{~s}, 9 \mathrm{H},{ }^{\mathrm{t}} \mathrm{Bu}\right) ;{ }^{13} \mathrm{C}$ NMR $\left(125 \mathrm{MHz}\right.$, Acetone- $\left.d_{6}\right) \delta$ 155.2, 154.3, 151.2, 136.4, 135.1, 131.9, 129.7, 128.6, 127.9, 126.0, 97.0, 95.7, 87.5, 80.4, 79.6, 77.4, 75.7, 74.8, 74.2, 61.2, 61.1, 52.2, 35.1, 31.7; HRMS (ESI) $m / z$ : found $[\mathrm{M}+\mathrm{Na}]^{+} 787.9951, \mathrm{C}_{29} \mathrm{H}_{33} \mathrm{Cl}_{6} \mathrm{NO}_{8} \mathrm{~S}$ calcd for $[\mathrm{M}+\mathrm{Na}]^{+} 787.9950$. 


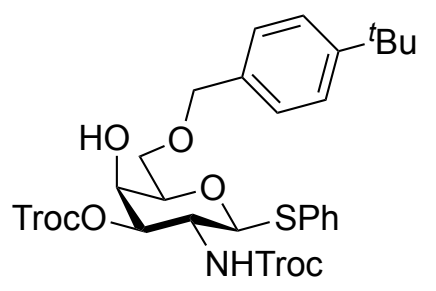

Phenyl 6-O-p-tert-butylbenzyl-2-deoxy-1-thio-2-(2,2,2-trichloroethoxycarbamoyl)-30 -(2,2,2-trichloroethoxycarbonyl)- $\beta$-D-galactopyranoside (9)

To a solution of compound $4 \mathrm{c}(47.8 \mathrm{mg}, 62.4 \mu \mathrm{mol})$ in $\mathrm{CH}_{2} \mathrm{Cl}_{2}(0.60 \mathrm{~mL})$ was added $4 \AA$ molecular sieves $(53.6 \mathrm{mg})$. After stirring for $30 \mathrm{~min}$ at ambient temperature, the reaction mixture was cooled to $-78{ }^{\circ} \mathrm{C}$ and added $\mathrm{Et}_{3} \mathrm{SiH}(29.8 \mu \mathrm{L}, 187 \mu \mathrm{mol})$ and $\mathrm{TfOH}(16.5 \mu \mathrm{L}, 187 \mu \mathrm{mol})$. After stirring for 20 $\min$ as the progress of the reaction was monitored by TLC $(n$-hexane/EtOAC $=2 / 1)$, the reaction was quenched with $\mathrm{Et}_{3} \mathrm{~N}$ and $\mathrm{MeOH}$. The reaction mixture was extracted with $\mathrm{CHCl}_{3}$ and washed with satd. aq. $\mathrm{NaHCO}_{3}$ and brine. The organic layer was dried over $\mathrm{Na}_{2} \mathrm{SO}_{4}$ and concentrated. The residue was purified by silica gel column chromatography ( $n$-hexane $\left./ \mathrm{CHCl}_{3} / \mathrm{EtOAc}=5 / 1 / 1\right)$ to give compound 9 (39.1 mg, 82\%) as a white amorphous solid; $[\alpha]_{\mathrm{D}}+6.2^{\circ}$ ( $\left.\mathrm{c} 0.7, \mathrm{CHCl}_{3}\right) ;{ }^{1} \mathrm{H} \mathrm{NMR}\left(500 \mathrm{MHz}\right.$, Acetone- $\left.d_{6}\right)$ ठ 7.55-7.24 (m, $9 \mathrm{H}, 2 \mathrm{Ar}$ ), 7.16 (d, $1 \mathrm{H}, J_{2, \mathrm{NH}}=9.5 \mathrm{~Hz}, \mathrm{NH}-2$ ), 5.13 (d, $1 \mathrm{H}, J_{1,2}=10.5 \mathrm{~Hz}, \mathrm{H}-1$ ), 5.05 (dd, $\left.1 \mathrm{H}, J_{3,4}=3.0 \mathrm{~Hz}, J_{2,3}=10.5 \mathrm{~Hz}, \mathrm{H}-3\right), 4.92\left(\mathrm{~d}, 1 \mathrm{H}, J_{\mathrm{gem}}=12.5 \mathrm{~Hz}, \mathrm{C}(\mathrm{O}) \mathrm{OCH}_{2} \mathrm{CCl}_{3}\right), 4.86(\mathrm{~d}, 1 \mathrm{H}$, $\left.\mathrm{C}(\mathrm{O}) \mathrm{OCH}_{2} \mathrm{CCl}_{3}\right), 4.83\left(\mathrm{~d}, 1 \mathrm{H}, \mathrm{J}_{\mathrm{gem}}=12.3 \mathrm{~Hz}, \mathrm{C}(\mathrm{O}) \mathrm{OCH}_{2} \mathrm{CCl}_{3}\right), 4.79\left(\mathrm{~d}, 1 \mathrm{H}, \mathrm{C}(\mathrm{O}) \mathrm{OCH}_{2} \mathrm{CCl}_{3}\right), 4.69(\mathrm{~d}, 1 \mathrm{H}$, $\left.J_{4, \mathrm{OH}}=5.5 \mathrm{~Hz}, \mathrm{OH}-4\right), 4.56-4.51\left(\mathrm{~m}, 1 \mathrm{H}, 2 \mathrm{ArCH}_{2}\right), 4.31$ (dd, $\left.1 \mathrm{H}, \mathrm{H}-4\right), 4.24$ (q, $1 \mathrm{H}, \mathrm{H}-2$ ), 3.94 (near t, $1 \mathrm{H}, \mathrm{H}-5$ ), 3.79 (dd, $1 \mathrm{H}, J_{5,6 a}=5.0 \mathrm{~Hz}, J_{\text {gem }}=10.0 \mathrm{~Hz}, \mathrm{H}-6 \mathrm{a}$ ), 3.75 (dd, $\left.1 \mathrm{H}, J_{5,6 b}=6.5 \mathrm{~Hz}, \mathrm{H}-6 \mathrm{~b}\right), 1.32$ (s, $\left.9 \mathrm{H},{ }^{\mathrm{t}} \mathrm{Bu}\right) ;{ }^{13} \mathrm{C}$ NMR $\left(125 \mathrm{MHz}\right.$, Acetone- $\left.d_{6}\right) \delta 155.3,154.2,151.0 .136 .6,135.6,131.7,129.8,128.3$, $127.9,125.9,97.0,95.6,87.9,80.2,78.2,77.5,74.8,73.6,70.2,66.9,51.8,35.0,31.7$; HRMS (ESI) $\mathrm{m} / \mathrm{z}$ : found $[\mathrm{M}+\mathrm{Na}]^{+} 787.9948, \mathrm{C}_{29} \mathrm{H}_{33} \mathrm{Cl}_{6} \mathrm{NO}_{8} \mathrm{~S}$ calcd for $[\mathrm{M}+\mathrm{Na}]^{+} 787.9950$.

\subsection{GIcNAc derivatives}

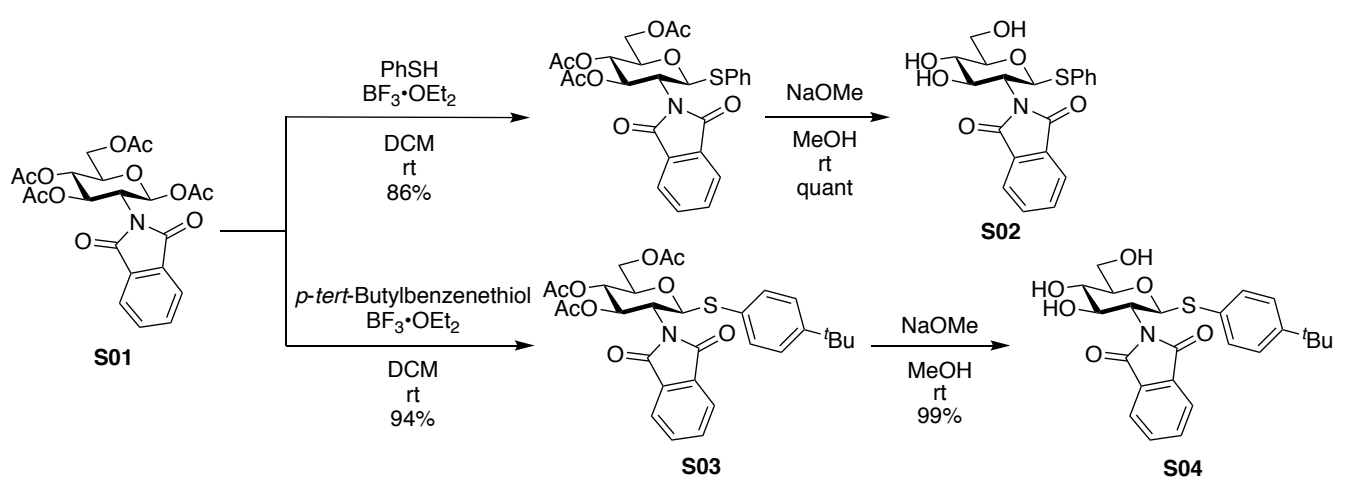

Supporting Scheme 1. Synthesis of GlcNPhth derivatives S02 and S04 


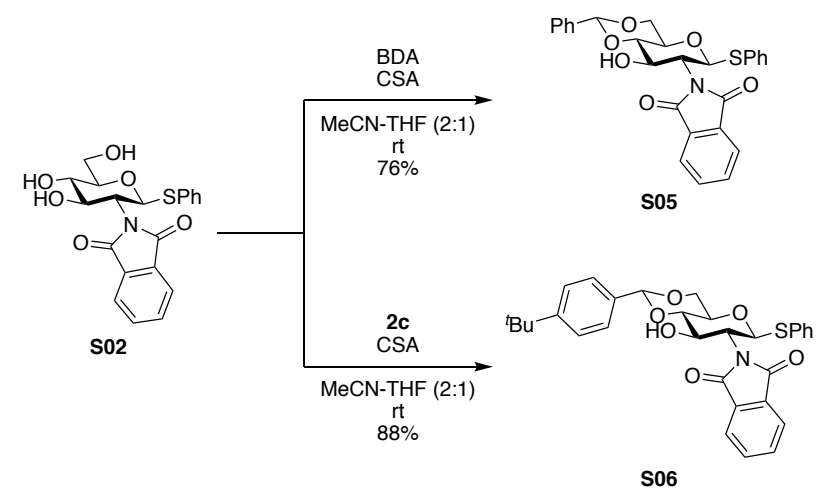

Supporting Scheme 2. Synthesis of GlcNPhth derivatives S05 $5^{\mathrm{S02}}$ and S06

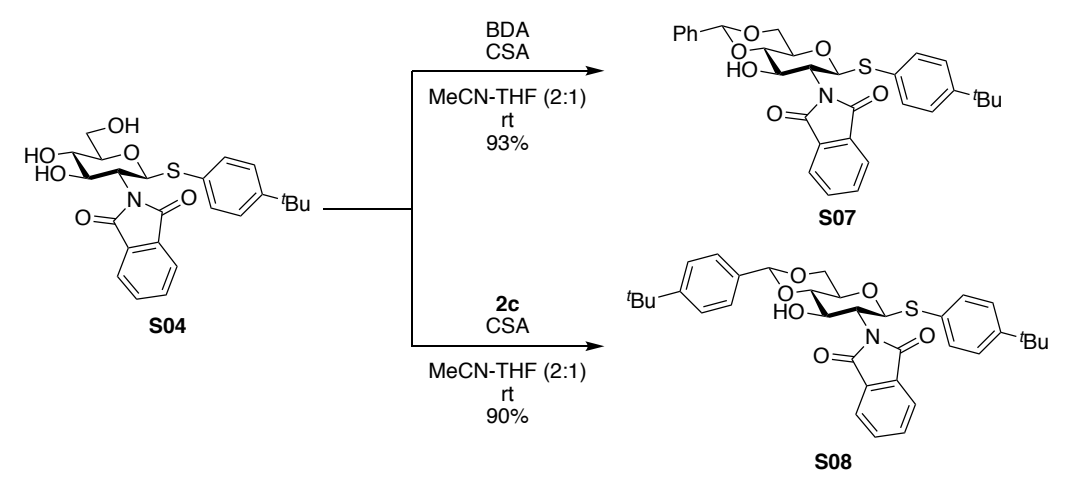

Supporting Scheme 3. Synthesis of GlcNPhth derivatives S07 and S08

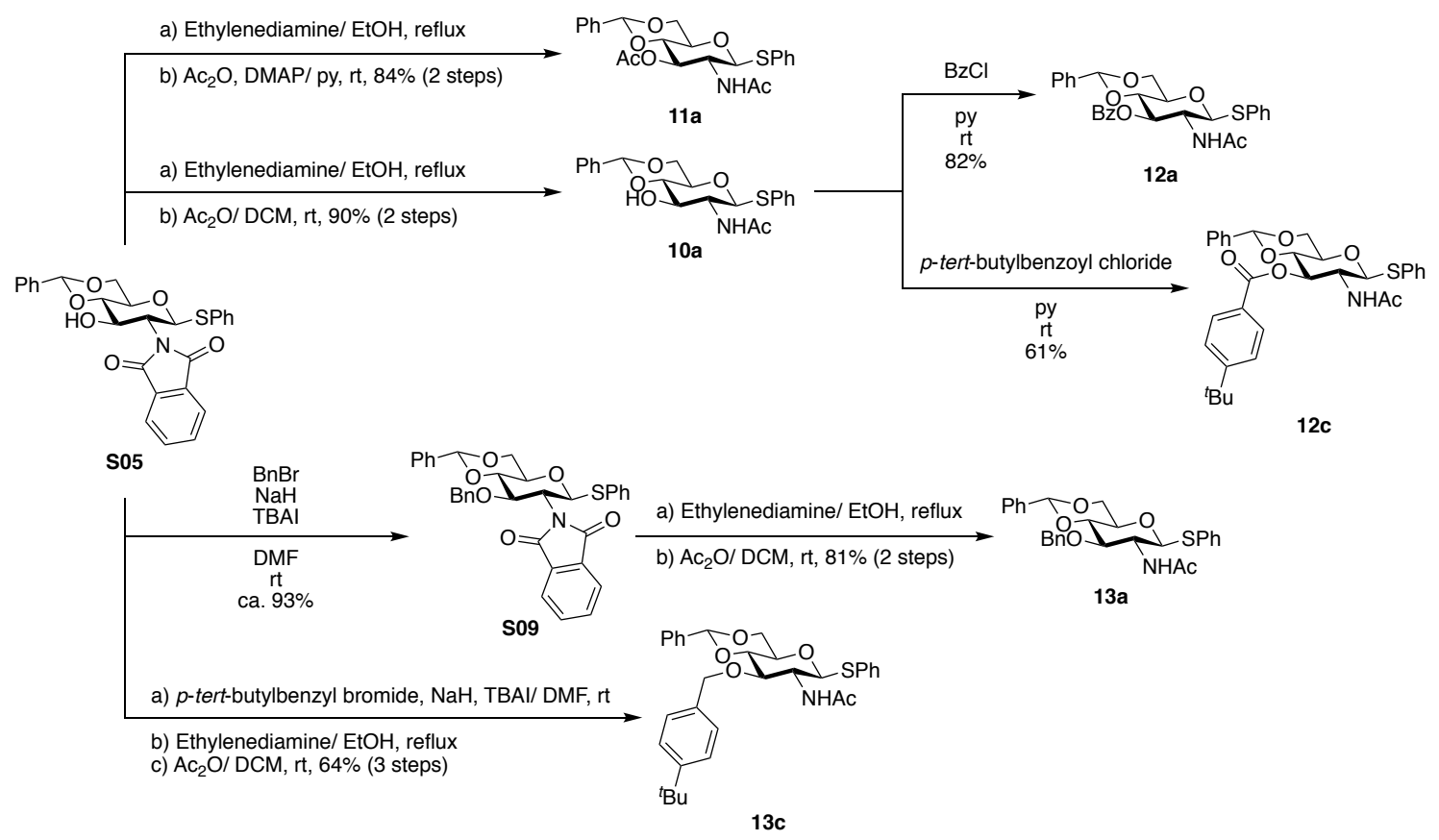

Supporting Scheme 4. Synthesis of GIcNAc derivatives $10 a^{503}, 11 a, 12 a, 12 c, 13 a$ and 


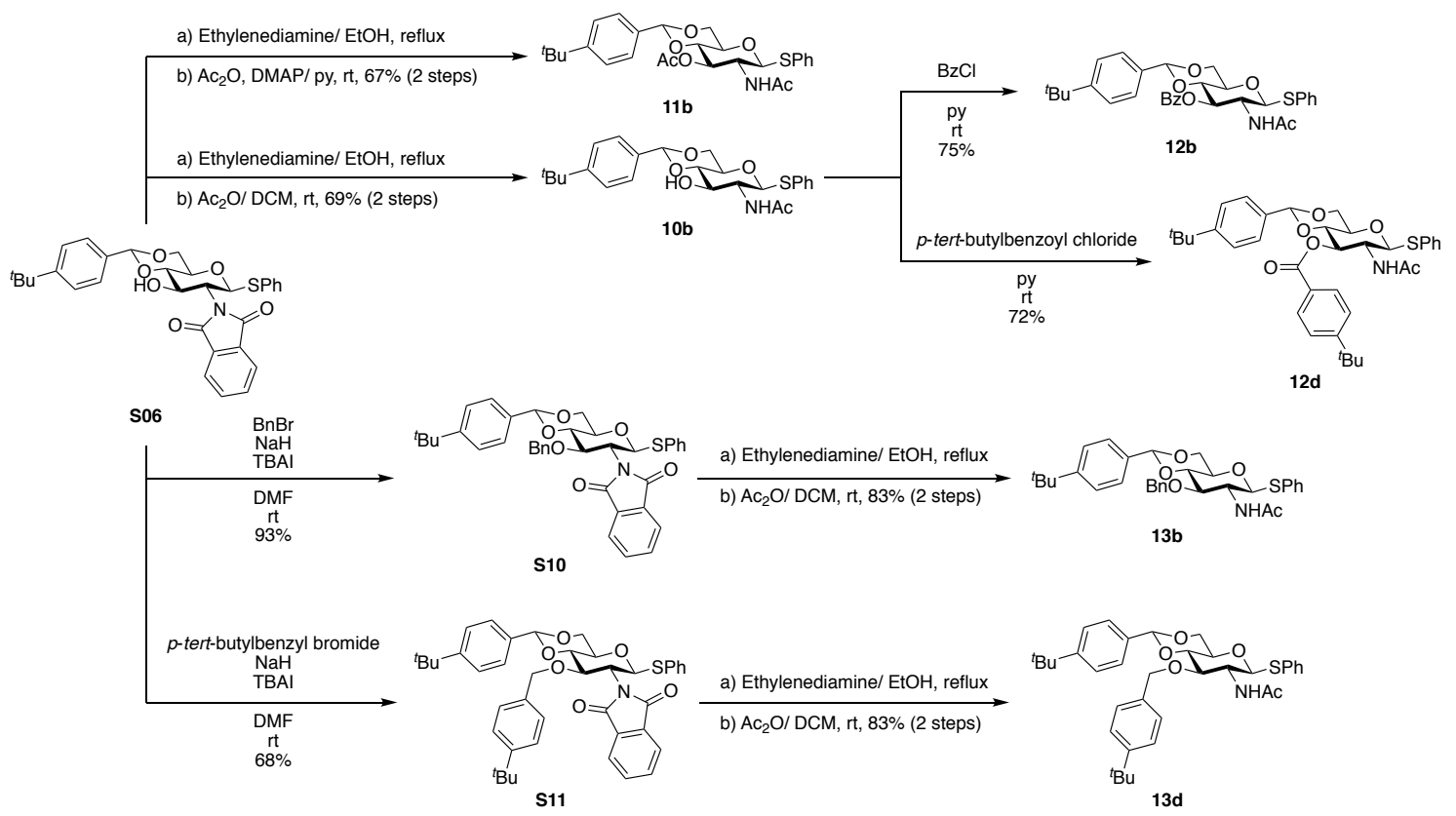

Supporting Scheme 5. Synthesis of GICNAc derivatives 10b, 11b, 12b, 12d, 13b and 13d

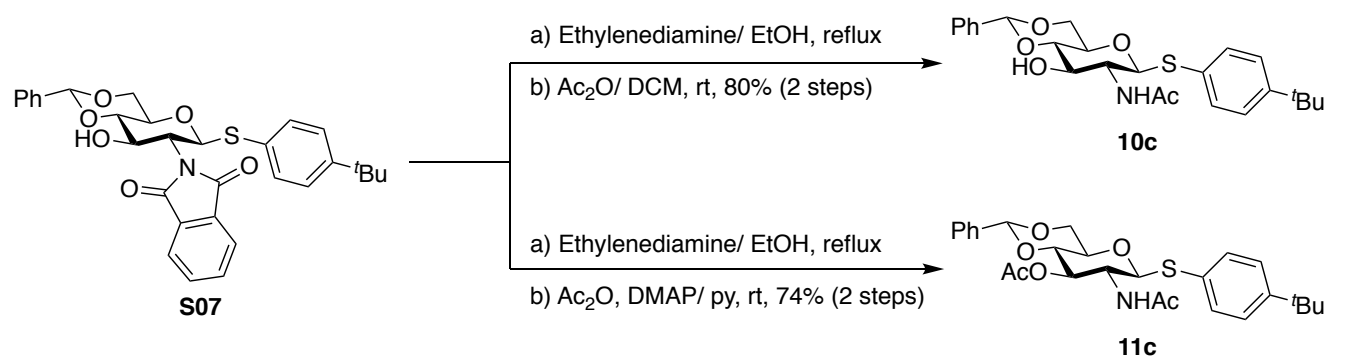

Supporting Scheme 6. Synthesis of GIcNAc derivatives 10c and 11c

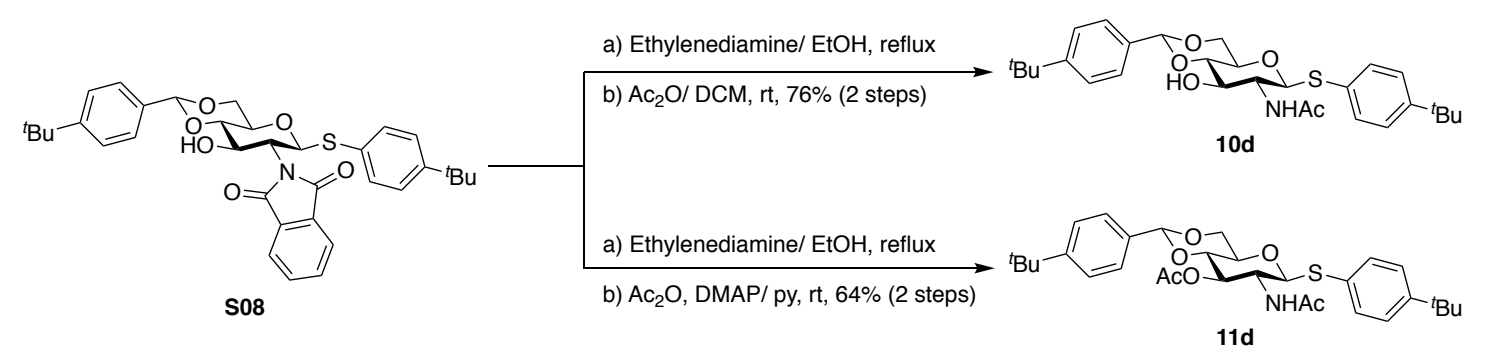

Supporting Scheme 7. Synthesis of GlcNAc derivatives 10d and 11d 


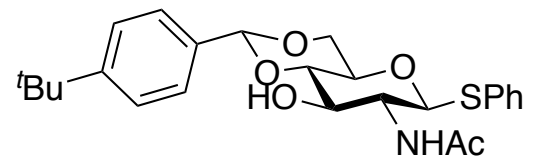

Phenyl 2-acetamido-4,6-O-p-tert-butylbenzylidene-2-deoxy-1-thio- $\beta$-Dglucopyranoside (10b)

To a solution of compound $\mathbf{S O 6}(600 \mathrm{mg}, 1.10 \mathrm{mmol})$ in EtOH $(11.0 \mathrm{~mL})$ was added ethylenediamine $(1.24 \mathrm{~mL}, 22.0 \mathrm{mmol})$. After stirring for $15 \mathrm{~h}$ as the progress of the reaction was monitored by TLC (toluene/EtOAc $=3 / 2$ ), the reaction mixture was neutralized with $2 \mathrm{M} \mathrm{HCl}(\mathrm{pH}<8)$ and extracted with $\mathrm{CHCl}_{3}$. The organic layer was washed with $\mathrm{H}_{2} \mathrm{O}$ and brine, dried over $\mathrm{Na}_{2} \mathrm{SO}_{4}$, concentrated, and dried for $2 \mathrm{~h}$. The residue was dissolved in $\mathrm{CH}_{2} \mathrm{Cl}_{2}\left(11.0 \mathrm{~mL}\right.$ ), followed by the addition of $\mathrm{Ac}_{2} \mathrm{O}$ (156 $\mu \mathrm{L}, 1.65$ $\mathrm{mmol}$ ). After stirring for $2 \mathrm{~h}$ at ambient temperature as the progress of the reaction was monitored by $\mathrm{TLC}\left(\mathrm{CHCl}_{3} / \mathrm{MeOH}=20 / 1\right)$, the reaction mixture was concentrated. The residue was crystallized ( $n$ -

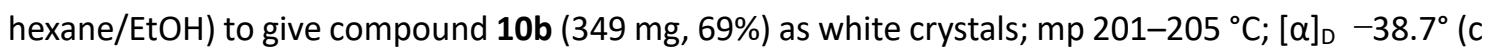
1.0, $\left.\mathrm{CHCl}_{3}\right) ;{ }^{1} \mathrm{H}$ NMR $\left(500 \mathrm{MHz}, \mathrm{CDCl}_{3}\right) \delta 7.52-7.32(\mathrm{~m}, 9 \mathrm{H}, 2 \mathrm{Ar}), 5.70\left(\mathrm{~d}, \mathrm{~J}_{2, \mathrm{NH}}=6.5 \mathrm{~Hz}, \mathrm{NH}-2\right), 5.53$ (s, $1 \mathrm{H}, \operatorname{ArCH<}$ ), 5.03 (d, J, J $=10.5 \mathrm{~Hz}, \mathrm{H}-1$ ), 4.36 (dd, $\left.1 \mathrm{H}, J_{5,6 \mathrm{a}}=4.8 \mathrm{~Hz}, J_{\text {gem }}=10.8 \mathrm{~Hz}, \mathrm{H}-6 \mathrm{a}\right), 4.11$ (td, $\left.1 \mathrm{H}, J_{3, \mathrm{OH}}=2.3 \mathrm{~Hz}, J_{2,3}=J_{3,4}=9.3 \mathrm{~Hz}, \mathrm{H}-3\right), 3.78\left(\mathrm{t}, 1 \mathrm{H}, J_{5,6 b}=10.0 \mathrm{~Hz}, \mathrm{H}-6 \mathrm{~b}\right), 3.61-3.50(\mathrm{~m}, 4 \mathrm{H}, \mathrm{H}-2$, $\mathrm{H}-4, \mathrm{H}-5, \mathrm{OH}-3), 2.09$ (s, $3 \mathrm{H}, \mathrm{Ac}), 1.29$ (s, $\left.9 \mathrm{H},{ }^{\mathrm{t}} \mathrm{Bu}\right) ;{ }^{13} \mathrm{C} \mathrm{NMR}\left(125 \mathrm{MHz}, \mathrm{CDCl}_{3}\right) \delta$ 171.5, 152.4, 134.1, $132.7,131.8,129.1,128.3,126.0,125.3,101.9,86.0,81.4,72.8,70.4,68.5,56.6,34.6,31.3$, 23.6; HRMS (ESI) $\mathrm{m} / \mathrm{z}$ : found [M+Na] ${ }^{+} 480.1815, \mathrm{C}_{25} \mathrm{H}_{31} \mathrm{NO}_{5} \mathrm{~S}$ calcd for $[\mathrm{M}+\mathrm{Na}]^{+} 480.1815$.

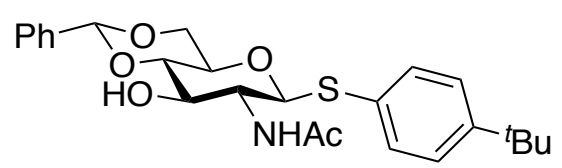

p-tert-Butylphenyl

2-acetamido-4,6-O-benzylidene-2-deoxy-1-thio- $\beta$-Dglucopyranoside (10c)

To a solution of compound $\mathbf{S 0 7}(200 \mathrm{mg}, 0.367 \mathrm{mmol})$ in EtOH $(3.70 \mathrm{~mL})$ was added ethylenediamine $(500 \mu \mathrm{L}, 7.33 \mathrm{mmol})$. After stirring for $24 \mathrm{~h}$ as the progress of the reaction was monitored by TLC (toluene/EtOAC $=1 / 1)$, the reaction mixture was neutralized with $2 \mathrm{M} \mathrm{HCl}(\mathrm{pH}<8)$ and extracted with $\mathrm{CHCl}_{3}$. The organic layer was washed with $\mathrm{H}_{2} \mathrm{O}$ and brine, dried over $\mathrm{Na}_{2} \mathrm{SO}_{4}$, concentrated, and dried for $2 \mathrm{~h}$. The residue was dissolved in $\mathrm{CH}_{2} \mathrm{Cl}_{2}(3.70 \mathrm{~mL})$, followed by the addition of $\mathrm{Ac}_{2} \mathrm{O}(52.1 \mu \mathrm{L}$, $0.550 \mathrm{mmol}$ ). After stirring for $2.5 \mathrm{~h}$ at ambient temperature as the progress of the reaction was monitored by $\mathrm{TLC}\left(\mathrm{CHCl}_{3} / \mathrm{MeOH}=10 / 1\right)$, the reaction mixture was concentrated. The residue was crystallized ( $n$-hexane/EtOAc) to give compound 10c (134 mg, 80\%) as white crystals; mp $242-248^{\circ} \mathrm{C}$; 
$[\alpha]_{D}-47.0^{\circ}$ (c 1.0, $\left.\mathrm{CHCl}_{3}\right) ;{ }^{1} \mathrm{H} \mathrm{NMR}\left(500 \mathrm{MHz}, \mathrm{CDCl}_{3}\right) \delta 7.48-7.33(\mathrm{~m}, 9 \mathrm{H}, 2 \mathrm{Ar}), 5.84\left(\mathrm{~d}, 1 \mathrm{H}, \mathrm{J}_{2, \mathrm{NH}}=\right.$ $7.0 \mathrm{~Hz}, \mathrm{NH}-2), 5.52\left(\mathrm{~s}, 1 \mathrm{H}, \mathrm{PhCH}\right.$ ) , $4.91\left(\mathrm{~d}, 1 \mathrm{H}, J_{1,2}=10.5 \mathrm{~Hz}, \mathrm{H}-1\right), 4.36\left(\mathrm{dd}, 1 \mathrm{H}, J_{5,6 \mathrm{a}}=4.3 \mathrm{~Hz}, J_{\text {gem }}=\right.$ $10.5 \mathrm{~Hz}, \mathrm{H}-6 \mathrm{a}), 4.07-4.04$ ( $\mathrm{m}, 2 \mathrm{H}, \mathrm{H}-3, \mathrm{OH}-3$ ), 3.77 (m, $1 \mathrm{H}, \mathrm{H}-6 \mathrm{~b}), 3.60-3.50$ (m, $3 \mathrm{H}, \mathrm{H}-2, \mathrm{H}-4, \mathrm{H}-5)$, $2.06(\mathrm{~s}, 3 \mathrm{H}, \mathrm{Ac}), 1.32\left(\mathrm{~s}, 9 \mathrm{H},{ }^{t} \mathrm{Bu}\right) ;{ }^{13} \mathrm{C}$ NMR $\left(125 \mathrm{MHz}, \mathrm{CDCl}_{3}\right) \delta 171.7,151.8,137.0,132.8,129.3$, 128.3, 127.9, 126.3, 126.2, 101.9, 86.1, 81.4, 72.8, 70.4, 68.5, 56.7, 34.7, 31.2, 23.6; HRMS (ESI) $m / z$ : found $[\mathrm{M}+\mathrm{Na}]^{+} 480.1815, \mathrm{C}_{25} \mathrm{H}_{31} \mathrm{NO}_{5} \mathrm{~S}$ calcd for $[\mathrm{M}+\mathrm{Na}]^{+} 480.1815$.

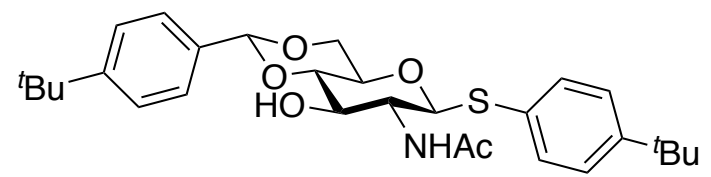

p-tert-Butylphenyl 2-acetamido-4,6-O-p-tert-butylbenzylidene-2-deoxy-1-thio- $\beta$-Dglucopyranoside (10d)

To a solution of compound $\mathbf{S 0 8}(200 \mathrm{mg}, 0.332 \mathrm{mmol})$ in EtOH $(3.30 \mathrm{~mL})$ was added ethylenediamine (450 $\mu \mathrm{L}, 6.65 \mathrm{mmol}$ ). After stirring for $24 \mathrm{~h}$ as the progress of the reaction was monitored by TLC (toluene/EtOAC $=1 / 1)$, the reaction mixture was neutralized with $2 \mathrm{M} \mathrm{HCl}(\mathrm{pH}<8)$ and extracted with $\mathrm{CHCl}_{3}$. The organic layer was washed with $\mathrm{H}_{2} \mathrm{O}$ and brine, dried over $\mathrm{Na}_{2} \mathrm{SO}_{4}$, concentrated, and dried for $2 \mathrm{~h}$. The residue was dissolved in $\mathrm{CH}_{2} \mathrm{Cl}_{2}(3.70 \mathrm{~mL})$, followed by the addition of $\mathrm{Ac}_{2} \mathrm{O}(47.3 \mu \mathrm{L}$, $0.499 \mathrm{mmol})$. After stirring for $2.5 \mathrm{~h}$ at ambient temperature as the progress of the reaction was monitored by $\mathrm{TLC}\left(\mathrm{CHCl}_{3} / \mathrm{MeOH}=10 / 1\right)$, the reaction mixture was concentrated. The residue was crystallized ( $n$-hexane/EtOH) to give compound $10 \mathrm{~d}\left(130 \mathrm{mg}, 76 \%\right.$ ) as white crystals; mp $236-240{ }^{\circ} \mathrm{C}$; $[\alpha]_{\mathrm{D}}-40.8^{\circ}\left(\mathrm{c} 1.0, \mathrm{CHCl}_{3}\right) ;{ }^{1} \mathrm{H}$ NMR $\left(500 \mathrm{MHz}, \mathrm{CDCl}_{3}\right) \delta 7.42-7.34(\mathrm{~m}, 8 \mathrm{H}, 2 \mathrm{Ar}), 5.75\left(\mathrm{~d}, 1 \mathrm{H}, \mathrm{J}_{2, \mathrm{NH}}=\right.$ $7.0 \mathrm{~Hz}, \mathrm{NH}-2$ ), $5.52\left(\mathrm{~s}, 1 \mathrm{H}, \mathrm{ArCH}<\right.$ ), $4.97\left(\mathrm{~d}, 1 \mathrm{H}, \mathrm{J}_{1,2}=10.5 \mathrm{~Hz}, \mathrm{H}-1\right), 4.37$ (dd, $1 \mathrm{H}, J_{5,6 a}=4.3 \mathrm{~Hz}, J_{\mathrm{gem}}=$ $10.3 \mathrm{~Hz}, \mathrm{H}-6 \mathrm{a}), 4.11\left(\mathrm{dt}, 1 \mathrm{H}, J_{3, \mathrm{OH}}=2.8 \mathrm{~Hz}, J_{2,3}=J_{3,4}=9.0 \mathrm{~Hz}, \mathrm{H}-3\right), 3.78\left(\mathrm{t}, 1 \mathrm{H}, J_{5,6 \mathrm{~b}}=10.0 \mathrm{~Hz}, \mathrm{H}-6 \mathrm{~b}\right)$, $3.71(\mathrm{~d}, 1 \mathrm{H}, \mathrm{OH}-3), 3.58-3.50$ (m, $3 \mathrm{H}, \mathrm{H}-2, \mathrm{H}-4, \mathrm{H}-5), 2.09$ (s, $3 \mathrm{H}, \mathrm{Ac}), 1.32\left(\mathrm{~s}, 9 \mathrm{H},{ }^{t} \mathrm{Bu}\right), 1.29$ (s, $9 \mathrm{H}$, $\left.{ }^{t} \mathrm{Bu}\right) ;{ }^{13} \mathrm{CNMR}\left(125 \mathrm{MHz}, \mathrm{CDCl}_{3}\right)$ ס 171.5, 152.3, 151.8, 134.1, 132.8, 127.9, 126.2, 126.0, 125.3, 102.0, 86.1, 81.4, 72.9, 70.4, 68.5, 56.8, 34.7, 31.3, 31.2, 23.6; HRMS (ESI) $m / z$ : found $[\mathrm{M}+\mathrm{Na}]^{+} 536.2442$, $\mathrm{C}_{29} \mathrm{H}_{39} \mathrm{NO}_{5} \mathrm{~S}$ calcd for $[\mathrm{M}+\mathrm{Na}]^{+} 536.2441$.

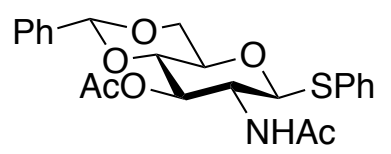

Phenyl

2-acetamido-3-O-acetyl-4,6-O-benzylidene-2-deoxy-1-thio- $\beta$-Dglucopyranoside (11a)

To a solution of compound $\mathbf{S 0 5}(298 \mathrm{mg}, 0.609 \mathrm{mmol})$ in EtOH $(12.2 \mathrm{~mL})$ was added ethylenediamine 
(296 $\mu \mathrm{L}, 6.09 \mathrm{mmol})$. After stirring for $7 \mathrm{~h}$ as the progress of the reaction was monitored by TLC (toluene/EtOAc $=1 / 1)$, the reaction mixture was neutralized with $2 \mathrm{M} \mathrm{HCl}(\mathrm{pH}<8)$ and extracted with $\mathrm{CHCl}_{3}$. The organic layer was washed with $\mathrm{H}_{2} \mathrm{O}$ and brine, dried over $\mathrm{Na}_{2} \mathrm{SO}_{4}$, concentrated, and dried for $2 \mathrm{~h}$. The residue was dissolved in pyridine $(5.50 \mathrm{~mL})$, followed by the addition of $\mathrm{Ac}_{2} \mathrm{O}(173 \mu \mathrm{L}$, $1.83 \mathrm{mmol}$ ) and DMAP $(7.4 \mathrm{mg}, 60.9 \mu \mathrm{mol})$. After stirring for $10 \mathrm{~h}$ at ambient temperature as the progress of the reaction was monitored by $\mathrm{TLC}\left(\mathrm{CHCl}_{3} / \mathrm{MeOH}=30 / 1\right)$, the reaction mixture was concentrated. The residue was crystallized (EtOH) to give compound $11 \mathrm{a}(228 \mathrm{mg}, 84 \%)$ as white crystals; mp >300 ${ }^{\circ} \mathrm{C} ;{ }^{1} \mathrm{H}$ NMR $\left(500 \mathrm{MHz}, \mathrm{CDCl}_{3}\right) \delta 7.48-7.30(\mathrm{~m}, 10 \mathrm{H}, 2 \mathrm{Ph}), 5.54\left(\mathrm{~d}, 1 \mathrm{H}, \mathrm{J}_{2, \mathrm{NH}}=9.5\right.$ $\mathrm{Hz}, \mathrm{NH}-2$ ), $5.52(\mathrm{~s}, 1 \mathrm{H}, \mathrm{PhCH}<), 5.22\left(\mathrm{t}, 1 \mathrm{H}, J_{2,3}=J_{3,4}=9.8 \mathrm{~Hz}, \mathrm{H}-3\right.$ ), $4.80\left(\mathrm{~d}, 1 \mathrm{H}, J_{1,2}=10.5 \mathrm{~Hz}, \mathrm{H}-1\right)$, $4.38\left(\mathrm{dd}, 1 \mathrm{H}, J_{5,6 a}=5.0 \mathrm{~Hz}, J_{\mathrm{gem}}=10.4 \mathrm{~Hz}, \mathrm{H}-6 \mathrm{a}\right.$ ), 4.19 (near q, $1 \mathrm{H}, \mathrm{H}-2$ ), $3.82\left(\mathrm{t}, 1 \mathrm{H}, J_{5,6 b}=10.3 \mathrm{~Hz}\right.$, H-6b), $3.72\left(t, 1 \mathrm{H}, J_{4,5}=9.5 \mathrm{~Hz}, \mathrm{H}-4\right), 3.56(\mathrm{~m}, 1 \mathrm{H}, \mathrm{H}-5), 2.07$ (s, $\left.3 \mathrm{H}, \mathrm{Ac}\right), 2.00(\mathrm{~s}, 3 \mathrm{H}, \mathrm{Ac})$.

* Compound is too insoluble to record specific rotation, ${ }^{13} \mathrm{C}$ NMR spectrum and HRMS.

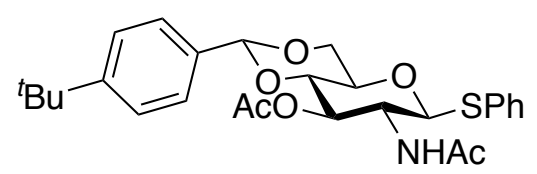

Phenyl 2-acetamido-3-O-acetyl-4,6-O-p-tert-butylbenzylidene-2-deoxy-1-thio- $\beta$-Dglucopyranoside (11b)

To a solution of compound $\mathbf{S 0 6}(300 \mathrm{mg}, 0.550 \mathrm{mmol})$ in EtOH $(5.50 \mathrm{~mL})$ was added ethylenediamine (743 $\mu \mathrm{L}, 11.0 \mathrm{mmol})$. After stirring for $13 \mathrm{~h}$ as the progress of the reaction was monitored by TLC (toluene/EtOAC $=3 / 2)$, the reaction mixture was neutralized with $2 \mathrm{M} \mathrm{HCl}(\mathrm{pH}<8)$ and extracted with $\mathrm{CHCl}_{3}$. The organic layer was washed with $\mathrm{H}_{2} \mathrm{O}$ and brine, dried over $\mathrm{Na}_{2} \mathrm{SO}_{4}$, concentrated, and dried for $2 \mathrm{~h}$. The residue was dissolved in pyridine $(5.50 \mathrm{~mL})$, followed by the addition of $\mathrm{Ac}_{2} \mathrm{O}(156 \mu \mathrm{L}$, $1.65 \mathrm{mmol}$ ) and DMAP $(6.7 \mathrm{mg}, 55.0 \mu \mathrm{mol})$. After stirring for $2 \mathrm{~h}$ at ambient temperature as the progress of the reaction was monitored by TLC (toluene/EtOAC $=1 / 1$ ), the reaction mixture was concentrated. The residue was crystallized (EtOH) to give compound $\mathbf{1 1 b}(184 \mathrm{mg}, 67 \%$ ) as white crystals; mp $243-246^{\circ} \mathrm{C} ;[\alpha]_{\mathrm{D}}-59.5^{\circ}$ (c 1.0, $\left.\mathrm{CHCl}_{3}\right) ;{ }^{1} \mathrm{H}$ NMR $\left(500 \mathrm{MHz}, \mathrm{CDCl}_{3}\right) \delta 7.48-7.22(\mathrm{~m}, 9 \mathrm{H}, 2$ $\operatorname{Ar}), 5.54\left(\mathrm{~d}, 1 \mathrm{H}, J_{2, \mathrm{NH}}=9.5 \mathrm{~Hz}, \mathrm{NH}-2\right), 5.50(\mathrm{~s}, 1 \mathrm{H}, \mathrm{ArCH}<), 5.21\left(\mathrm{t}, 1 \mathrm{H}, J_{2,3}=J_{3,4}=9.8 \mathrm{~Hz}, \mathrm{H}-3\right), 4.80$ (d, $1 \mathrm{H}, J_{1,2}=10.5 \mathrm{~Hz}, \mathrm{H}-1$ ), 4.35 (dd, $1 \mathrm{H}, J_{5,6 \mathrm{a}}=4.8 \mathrm{~Hz}, J_{\text {gem }}=10.5 \mathrm{~Hz}, \mathrm{H}-6 \mathrm{a}$ ), 4.18 (near q, $1 \mathrm{H}, \mathrm{H}-2$ ), $3.80\left(\mathrm{t}, 1 \mathrm{H}, J_{5,6 b}=10.8 \mathrm{~Hz}, \mathrm{H}-6 \mathrm{~b}\right), 3.71\left(\mathrm{t}, 1 \mathrm{H}, J_{4,5}=9.5 \mathrm{~Hz}, \mathrm{H}-4\right), 3.55(\mathrm{~m}, 1 \mathrm{H}, \mathrm{H}-5), 2.06(\mathrm{~s}, 3 \mathrm{H}, \mathrm{Ac})$, $2.00(\mathrm{~s}, 3 \mathrm{H}, \mathrm{Ac}), 1.30$ (s, $\left.9 \mathrm{H},{ }^{\mathrm{t} B u}\right) ;{ }^{13} \mathrm{C} \mathrm{NMR}\left(125 \mathrm{MHz}, \mathrm{CDCl}_{3}\right) \delta 171.3,170.0,152.2,134.0,132.7$, 132.2, 129.0, 128.0, 125.8, 125.2, 101.5, 88.0, 78.2, 73.1, 70.8, 68.5, 53.5, 34.6, 31.3, 23.3, 20.9; HRMS (ESI) $\mathrm{m} / z$ : found $[\mathrm{M}+\mathrm{Na}]^{+} 522.1921, \mathrm{C}_{27} \mathrm{H}_{33} \mathrm{NO}_{6} \mathrm{~S}$ calcd for $[\mathrm{M}+\mathrm{Na}]^{+} 522.1921$. 


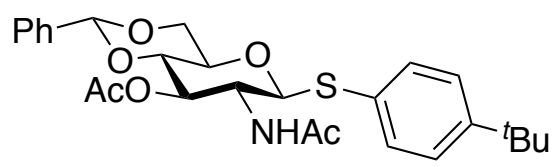

p-tert-Butylphenyl 2-acetamido-3-O-acetyl-4,6-O-benzylidene-2-deoxy-1-thio- $\beta$-Dglucopyranoside (11c)

To a solution of compound $\mathbf{S 0 7}(298 \mathrm{mg}, 0.546 \mathrm{mmol})$ in EtOH $(5.50 \mathrm{~mL})$ was added ethylenediamine (736 $\mu \mathrm{L}, 10.9 \mathrm{mmol})$. After stirring for $17 \mathrm{~h}$ as the progress of the reaction was monitored by TLC (toluene/EtOAC $=3 / 2)$, the reaction mixture was neutralized with $2 \mathrm{M} \mathrm{HCl}(\mathrm{pH}<8)$ and extracted with $\mathrm{CHCl}_{3}$. The organic layer was washed with $\mathrm{H}_{2} \mathrm{O}$ and brine, dried over $\mathrm{Na}_{2} \mathrm{SO}_{4}$, concentrated, and dried for $2 \mathrm{~h}$. The residue was dissolved in pyridine $(5.50 \mathrm{~mL})$, followed by the addition of $\mathrm{Ac}_{2} \mathrm{O}(155 \mu \mathrm{L}$, $1.64 \mathrm{mmol}$ ) and DMAP (6.7 mg, $54.6 \mu \mathrm{mol})$. After stirring for $2 \mathrm{~h}$ at ambient temperature as the progress of the reaction was monitored by TLC (toluene/EtOAC $=1 / 1$ ), the reaction mixture was concentrated. The residue was crystallized ( $n$-hexane/ $\mathrm{CHCl}_{3}$ ) to give compound $11 \mathrm{c}$ ( $201 \mathrm{mg}, 74 \%$ ) as white crystals; mp $282-285^{\circ} \mathrm{C}$; $[\alpha]_{\mathrm{D}}-35.3^{\circ}$ (c 1.0, $\left.\mathrm{CHCl}_{3}\right) ;{ }^{1} \mathrm{H} \mathrm{NMR}\left(500 \mathrm{MHz}, \mathrm{CDCl}_{3}\right) \delta 7.43-7.32(\mathrm{~m}$, $9 \mathrm{H}, 2 \mathrm{Ar}$ ), $5.75\left(\mathrm{~d}, 1 \mathrm{H}, J_{2, \mathrm{NH}}=10.0 \mathrm{~Hz}, \mathrm{NH}-2\right), 5.50(\mathrm{~s}, 1 \mathrm{H}, \mathrm{PhCH}<), 5.22\left(\mathrm{t}, 1 \mathrm{H}, J_{2,3}=J_{3,4}=9.8 \mathrm{~Hz}, \mathrm{H}-\right.$ 3), $4.76\left(d, 1 \mathrm{H}, J_{1,2}=10.5 \mathrm{~Hz}, \mathrm{H}-1\right.$ ), 4.34 (dd, $1 \mathrm{H}, J_{5,6 \mathrm{a}}=5.0 \mathrm{~Hz}, J_{\mathrm{gem}}=10.5 \mathrm{~Hz}, \mathrm{H}-6 \mathrm{a}$ ), 4.20 (near q, 1 $\mathrm{H}, \mathrm{H}-2$ ), 3.80 (t, $\left.1 \mathrm{H}, J_{5,6 b}=10.3 \mathrm{~Hz}, \mathrm{H}-6 \mathrm{~b}\right), 3.72\left(\mathrm{t}, 1 \mathrm{H}, \mathrm{J}_{4,5}=9.5 \mathrm{~Hz}, \mathrm{H}-4\right), 3.54(\mathrm{~m}, 1 \mathrm{H}, \mathrm{H}-5), 2.07$ (s, 3 $\mathrm{H}, \mathrm{Ac}), 2.00(\mathrm{~s}, 3 \mathrm{H}, \mathrm{Ac}), 1.31\left(\mathrm{~s}, 9 \mathrm{H},{ }^{\mathrm{t} B u}\right) ;{ }^{13} \mathrm{C}$ NMR (125 MHz, $\left.\mathrm{CDCl}_{3}\right) \delta$ 171.4, 170.1, 151.3, 136.9, 132.2, 129.2, 128.2, 126.2, 126.1, 101.4, 88.3, 78.3, 73.1, 70.8, 68.5, 53.6, 34.6, 31.2, 23.4, 20.9; HRMS (ESI) $m / z$ : found $[\mathrm{M}+\mathrm{Na}]^{+} 522.1921, \mathrm{C}_{27} \mathrm{H}_{33} \mathrm{NO}_{6} \mathrm{~S}$ calcd for $[\mathrm{M}+\mathrm{Na}]^{+} 522.1921$.

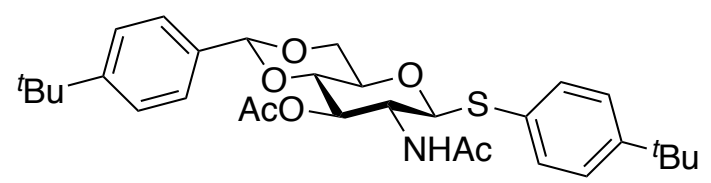

p-tert-Butylphenyl 2-acetamido-3-O-acetyl-4,6-O-p-tert-butylbenzylidene-2-deoxy-1thio- $\beta$-D-glucopyranoside (11d)

To a solution of compound $\mathbf{S 0 8}(323 \mathrm{mg}, 0.537 \mathrm{mmol})$ in EtOH $(5.40 \mathrm{~mL})$ was added ethylenediamine (723 $\mu \mathrm{L}, 10.7 \mathrm{mmol})$. After stirring for $17 \mathrm{~h}$ as the progress of the reaction was monitored by TLC (toluene/EtOAc $=3 / 2$ ), the reaction mixture was neutralized with $2 \mathrm{M} \mathrm{HCl}(\mathrm{pH}<8)$ and extracted with $\mathrm{CHCl}_{3}$. The organic layer was washed with $\mathrm{H}_{2} \mathrm{O}$ and brine, dried over $\mathrm{Na}_{2} \mathrm{SO}_{4}$, concentrated, and dried for $2 \mathrm{~h}$. The residue was dissolved in pyridine $(5.40 \mathrm{~mL})$, followed by the addition of $\mathrm{Ac}_{2} \mathrm{O}(152 \mu \mathrm{L}$, $1.61 \mathrm{mmol}$ ) and DMAP $(6.6 \mathrm{mg}, 53.7 \mu \mathrm{mol})$. After stirring for $2 \mathrm{~h}$ at ambient temperature as the 
progress of the reaction was monitored by TLC (toluene/EtOAC $=1 / 1$ ), the reaction mixture was concentrated. The residue was crystallized ( $n$-hexane/EtOAc) to give compound 11d (192 mg, 64\%) as white crystals; $\mathrm{mp} 223-226^{\circ} \mathrm{C} ;[\alpha]_{\mathrm{D}}-33.7^{\circ}$ (c $1.0, \mathrm{CHCl}_{3}$ ); ${ }^{1} \mathrm{H} \mathrm{NMR}\left(500 \mathrm{MHz}, \mathrm{CDCl}_{3}\right) \delta 7.41-7.32$ $(\mathrm{m}, 8 \mathrm{H}, 2 \mathrm{Ar}), 5.70\left(\mathrm{~d}, 1 \mathrm{H}, J_{\mathrm{NH}, 2}=10.0 \mathrm{~Hz}, \mathrm{NH}-2\right), 5.49(\mathrm{~s}, 1 \mathrm{H}, \operatorname{ArCH}<), 5.21\left(\mathrm{t}, 1 \mathrm{H}, J_{2,3}=J_{3,4}=9.8 \mathrm{~Hz}\right.$, $\mathrm{H}-3$ ), $4.75\left(\mathrm{~d}, 1 \mathrm{H}, J_{1,2}=10.5 \mathrm{~Hz}, \mathrm{H}-1\right), 4.33\left(\mathrm{dd}, 1 \mathrm{H}, J_{5,6 \mathrm{a}}=5.0 \mathrm{~Hz}, J_{\mathrm{gem}}=10.4 \mathrm{~Hz}, \mathrm{H}-6 \mathrm{a}\right), 4.19$ (q, $1 \mathrm{H}$, $\mathrm{H}-2), 3.80$ (t, $\left.1 \mathrm{H}, \mathrm{J}_{5,6 \mathrm{~b}}=10.3 \mathrm{~Hz}, \mathrm{H}-6 \mathrm{~b}\right), 3.71\left(\mathrm{t}, 1 \mathrm{H}, \mathrm{J}_{4,5}=9.5 \mathrm{~Hz}, \mathrm{H}-4\right), 3.54(\mathrm{~m}, 1 \mathrm{H}, \mathrm{H}-5), 2.06(\mathrm{~s}, 3 \mathrm{H}$, Ac), $2.00(\mathrm{~s}, 3 \mathrm{H}, \mathrm{Ac}), 1.31\left(\mathrm{~s}, 9 \mathrm{H},{ }^{t} \mathrm{Bu}\right), 1.29\left(\mathrm{~s}, 9 \mathrm{H},{ }^{\mathrm{t}} \mathrm{Bu}\right) ;{ }^{13} \mathrm{C} \mathrm{NMR}\left(125 \mathrm{MHz}, \mathrm{CDCl}_{3}\right) \delta$ 171.3, 170.0, $152.2,151.3,134.0,132.2,129.2,126.0,125.9,125.2,101.5,88.3,78.2,73.1,70.8,68.5,53.6,34.6$, 34.6, 31.3, 31.2, 23.4, 20.9; HRMS (ESI) $m / z$ : found $[\mathrm{M}+\mathrm{Na}]^{+} 578.2547, \mathrm{C}_{31} \mathrm{H}_{41} \mathrm{NO}_{6} \mathrm{~S}$ calcd for $[\mathrm{M}+\mathrm{Na}]^{+}$ 578.2547.

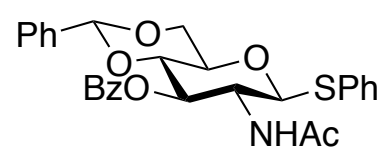

Phenyl 2-acetamido-3-O-benzoyl-4,6-O-benzylidene-2-deoxy-1-thio- $\beta$-Dglucopyranoside (12a)

To a solution of compound 10a (206 mg, $0.513 \mathrm{mmol})$ in pyridine $(5.10 \mathrm{~mL})$ was added $\mathrm{BzCl}(89.5 \mu \mathrm{L}$, $0.770 \mathrm{mmol}$ ) at $0{ }^{\circ} \mathrm{C}$. After stirring for $7 \mathrm{~h}$ at ambient temperature as the progress of the reaction was monitored by $\mathrm{TLC}\left(\mathrm{CHCl}_{3} / \mathrm{MeOH}=20 / 1\right)$, the reaction mixture was co-evaporated with toluene. The residue was crystallized (EtOH) to give compound 12a ( $212 \mathrm{mg}, 82 \%$ ) as white crystals; $\mathrm{mp}>300^{\circ} \mathrm{C}$; ${ }^{1} \mathrm{H}$ NMR $\left(500 \mathrm{MHz}, \mathrm{CDCl}_{3}\right) \delta 8.02-7.29(\mathrm{~m}, 15 \mathrm{H}, 3 \mathrm{Ph}), 5.66\left(\mathrm{~d}, 1 \mathrm{H}, \mathrm{J}_{2, \mathrm{NH}}=9.5 \mathrm{~Hz}, \mathrm{NH}-2\right), 5.55(\mathrm{~s}, 1 \mathrm{H}$, $\mathrm{PhCH}<$ ), $5.51\left(\mathrm{t}, 1 \mathrm{H}, J_{2,3}=J_{3,4}=9.8 \mathrm{~Hz}, \mathrm{H}-3\right), 4.91\left(\mathrm{~d}, 1 \mathrm{H}, J_{1,2}=10.5 \mathrm{~Hz}, \mathrm{H}-1\right), 4.41\left(\mathrm{dd}, 1 \mathrm{H}, J_{5.6 a}=5.2\right.$ $\left.\mathrm{Hz}, J_{\text {gem }}=10.5 \mathrm{~Hz}, \mathrm{H}-6 \mathrm{a}\right), 4.36$ (near q, $\left.1 \mathrm{H}, \mathrm{H}-2\right), 3.90\left(\mathrm{t}, 1 \mathrm{H}, J_{4,5}=9.5 \mathrm{~Hz}, \mathrm{H}-4\right), 3.87\left(\mathrm{t}, 1 \mathrm{H}, J_{5,6 b}=10.0\right.$ $\mathrm{Hz}, \mathrm{H}-6 \mathrm{~b}), 3.66$ (m, $1 \mathrm{H}, \mathrm{H}-5), 1.90$ (s, $3 \mathrm{H}, \mathrm{Ac}) ;{ }^{13} \mathrm{C} \mathrm{NMR}\left(125 \mathrm{MHz}, \mathrm{CDCl}_{3}\right)$ \& 170.0, 167.0, 136.8, 133.5, 133.0, 132.1, 129.9, 129.2, 129.0, 128.5, 128.2, 127.9, 126.1, 101.4, 88.2, 78.6, 73.5, 70.9, 68.5, 53.7, 23.3.

* Compound is too insoluble to record specific rotation and HRMS.

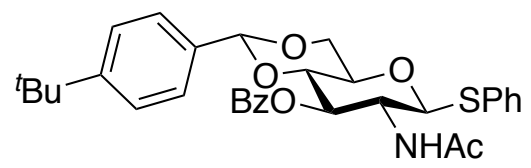

Phenyl 2-acetamido-3-O-benzoyl-4,6-O-p-tert-butylbenzylidene-2-deoxy-1-thio- $\beta$-Dglucopyranoside (12b)

To a solution of compound $10 \mathrm{~b}$ (167 mg, $0.365 \mathrm{mmol}$ ) in pyridine $(3.70 \mathrm{~mL}$ ) was added $\mathrm{BzCl}(63.5 \mu \mathrm{L}$, 
$0.547 \mathrm{mmol}$ ) at $0{ }^{\circ} \mathrm{C}$. After stirring for $4 \mathrm{~h}$ at ambient temperature as the progress of the reaction was monitored by $\mathrm{TLC}\left(\mathrm{CHCl}_{3} / \mathrm{MeOH}=20 / 1\right)$, the reaction mixture was co-evaporated with toluene. The residue was crystallized ( $n$-hexane/EtOH) to give compound $\mathbf{1 2 b}$ (153 mg, 75\%) as white crystals; mp $232-236^{\circ} \mathrm{C} ;[\alpha]_{\mathrm{D}}-53.8^{\circ}$ (c 1.0, $\left.\mathrm{CHCl}_{3}\right) ;{ }^{1} \mathrm{H}$ NMR $\left(500 \mathrm{MHz}, \mathrm{CDCl}_{3}\right)$ 8 8.01-7.32 (m, $14 \mathrm{H}, 3 \mathrm{Ar}$ ), 5.68 (d, $\left.1 \mathrm{H}, J_{2, \mathrm{NH}}=9.5 \mathrm{~Hz}, \mathrm{NH}-2\right), 5.53(\mathrm{~s}, 1 \mathrm{H}, \mathrm{ArCH}<), 5.51\left(\mathrm{t}, 1 \mathrm{H}, J_{2,3}=J_{3,4}=9.8 \mathrm{~Hz}, \mathrm{H}-3\right), 4.91\left(\mathrm{~d}, 1 \mathrm{H}, J_{1,2}=\right.$ $10.5 \mathrm{~Hz}, \mathrm{H}-1$ ), 4.40-4.33 (m, $2 \mathrm{H}, \mathrm{H}-2, \mathrm{H}-6 \mathrm{a}), 3.91-3.83$ (m, $2 \mathrm{H}, \mathrm{H}-4, \mathrm{H}-6 \mathrm{~b}), 3.64$ (m, $1 \mathrm{H}, \mathrm{H}-5), 1.89$ (s, $3 \mathrm{H}, \mathrm{Ac}), 1.26$ (s, $\left.9 \mathrm{H},{ }^{\mathrm{t}} \mathrm{Bu}\right) ;{ }^{13} \mathrm{C}$ NMR $\left(125 \mathrm{MHz}, \mathrm{CDCl}_{3}\right) \delta$ 170.0, 167.0, 152.1, 134.0, 133.5, 133.0, 132.1, $129.9,129.2$, 129.0, 128.5, 127.9, 125.8, 125.2, 101.5, 88.2, 78.6, 73.6, 71.0, 68.5, 53.7, 34.6, 31.2, 23.3; HRMS (ESI) $m / z$ : found [M+Na] $]^{+} 584.2078, \mathrm{C}_{32} \mathrm{H}_{35} \mathrm{NO}_{6} \mathrm{~S}$ calcd for $[\mathrm{M}+\mathrm{Na}]^{+} 584.2077$.

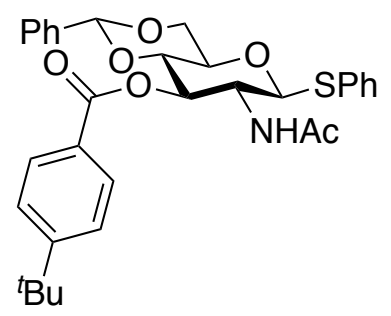

Phenyl 2-acetamido-4,6-O-benzylidene-3-O-p-tert-butylbenzoyl-2-deoxy-1-thio- $\beta$-Dglucopyranoside (12c)

To a solution of compound 10a $(210 \mathrm{mg}, 0.523 \mathrm{mmol})$ in pyridine $(5.20 \mathrm{~mL})$ was added p-tertbutylbenzoyl chloride (142 $\mu \mathrm{L}, 0.785 \mathrm{mmol})$. After stirring for $2.5 \mathrm{~h}$ at ambient temperature as the progress of the reaction was monitored by $\mathrm{TLC}\left(\mathrm{CHCl}_{3} / \mathrm{MeOH}=20 / 1\right)$, the reaction mixture was coevaporated with toluene. The residue was crystallized ( $n$-hexane/ $\mathrm{CHCl}_{3}$ ) to give compound $12 \mathrm{c}$ (179 $\mathrm{mg}, 61 \%$ ) as white crystals; mp $243-247^{\circ} \mathrm{C} ;[\alpha]_{\mathrm{D}}-32.2^{\circ}$ (c $\left.0.6, \mathrm{CHCl}_{3}\right) ;{ }^{1} \mathrm{H} \mathrm{NMR}\left(500 \mathrm{MHz}, \mathrm{CDCl}_{3}\right) \delta$ 7.94-7.24 (m, $14 \mathrm{H}, 3 \mathrm{Ar}), 5.91\left(\mathrm{~d}, 1 \mathrm{H}, J_{2, \mathrm{NH}}=9.5 \mathrm{~Hz}, \mathrm{NH}-2\right), 5.56-5.53(\mathrm{~m}, 2 \mathrm{H}, \mathrm{H}-3, \mathrm{PhCH}<), 4.91$ (d, $1 \mathrm{H}, J_{1,2}=10.5 \mathrm{~Hz}, \mathrm{H}-1$ ), 4.39 (near q, $1 \mathrm{H}, \mathrm{H}-2$ ), 4.32 (dd, $1 \mathrm{H}, J_{5,6 \mathrm{a}}=5.0 \mathrm{~Hz}, J_{\text {gem }}=10.4 \mathrm{~Hz}, \mathrm{H}-6 \mathrm{a}$ ), 3.88 $\left(\mathrm{t}, 1 \mathrm{H}, J_{3,4}=J_{4,5}=9.5 \mathrm{~Hz}, \mathrm{H}-4\right), 3.83\left(\mathrm{t}, 1 \mathrm{H}, J_{5,6 b}=10.3 \mathrm{~Hz}, \mathrm{H}-6 \mathrm{~b}\right), 3.64(\mathrm{~m}, 1 \mathrm{H}, \mathrm{H}-5), 1.89(\mathrm{~s}, 3 \mathrm{H}, \mathrm{Ac})$, 1.32 (s, $\left.9 \mathrm{H},{ }^{t} \mathrm{Bu}\right) ;{ }^{13} \mathrm{C}$ NMR $\left(125 \mathrm{MHz}, \mathrm{CDCl}_{3}\right) \delta 170.1,167.0,157.3,136.8,133.1,132.0,129.8,129.0$, $128.2,127.9,126.4,126.1,125.5,101.4,88.3,78.6,73.3,70.9,68.5,53.7,35.1,31.1,23.3$; HRMS (ESI) $\mathrm{m} / \mathrm{z}$ : found $[\mathrm{M}+\mathrm{Na}]^{+} 584.2079, \mathrm{C}_{32} \mathrm{H}_{35} \mathrm{NO}_{6} \mathrm{~S}$ calcd for $[\mathrm{M}+\mathrm{Na}]^{+} 584.2077$. 


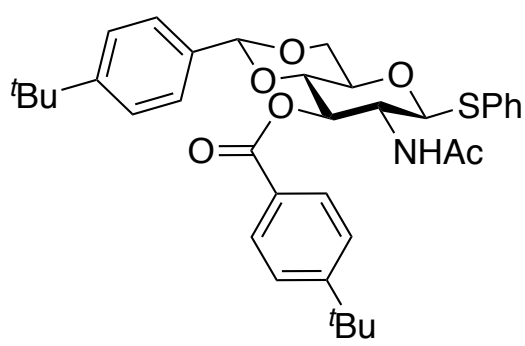

Phenyl 2-acetamido-3-O-p-tert-butylbenzoyl-4,6-0-p-tert-butylbenzylidene-2-deoxy1-thio- $\beta$-D-glucopyranoside (12d)

To a solution of compound $10 \mathrm{~b}(186 \mathrm{mg}, 0.406 \mathrm{mmol})$ in pyridine $(4.10 \mathrm{~mL})$ was added $p$-tertbutylbenzoyl chloride $(110 \mu \mathrm{L}, 0.610 \mathrm{mmol})$ at $0{ }^{\circ} \mathrm{C}$. After stirring for $4 \mathrm{~h}$ at ambient temperature as the progress of the reaction was monitored by $\mathrm{TLC}\left(\mathrm{CHCl}_{3} / \mathrm{MeOH}=20 / 1\right)$, the reaction mixture was co-evaporated with toluene. The residue was purified by silica gel column chromatography $\left(\mathrm{CHCl}_{3} /\right.$ EtOAc $\left.=30 / 1\right)$ and crystallized ( $n$-hexane/EtOAc) to give compound $\mathbf{1 2 d}(181 \mathrm{mg}, 72 \%)$ as white crystals; mp 187-193 ${ }^{\circ} \mathrm{C}$; $[\alpha]_{\mathrm{D}}-62.7^{\circ}$ (c 1.0, $\left.\mathrm{CHCl}_{3}\right) ;{ }^{1} \mathrm{H}$ NMR $\left(500 \mathrm{MHz}, \mathrm{CDCl}_{3}\right) \delta$ 7.94-7.28 (m, $13 \mathrm{H}, 3 \mathrm{Ar}$ ), $5.69\left(\mathrm{~d}, 1 \mathrm{H}, J_{2, \mathrm{NH}}=9.5 \mathrm{~Hz}, \mathrm{NH}-2\right), 5.53(\mathrm{~s}, 1 \mathrm{H}, \mathrm{ArCH}<), 5.49\left(\mathrm{t}, 1 \mathrm{H}, J_{2,3}=J_{3,4}=9.8 \mathrm{~Hz}, \mathrm{H}-\right.$ 3), 4.90 (d, $1 \mathrm{H}, J_{1,2}=10.5 \mathrm{~Hz}, \mathrm{H}-1$ ), 4.39-4.32 (m, $\left.2 \mathrm{H}, \mathrm{H}-2, \mathrm{H}-6 \mathrm{a}\right), 3.88\left(\mathrm{t}, 1 \mathrm{H}, J_{4,5}=9.5 \mathrm{~Hz}, \mathrm{H}-4\right), 3.85$ $\left(\mathrm{t}, 1 \mathrm{H}, J_{5,6 b}=J_{\text {gem }}=10.3 \mathrm{~Hz}, \mathrm{H}-6 \mathrm{~b}\right), 3.64(\mathrm{~m}, 1 \mathrm{H}, \mathrm{H}-5), 1.89(\mathrm{~s}, 3 \mathrm{H}, \mathrm{Ac}), 1.32\left(\mathrm{~s}, 9 \mathrm{H},{ }^{t} \mathrm{Bu}\right), 1.26(\mathrm{~s}, 9 \mathrm{H}$, $\left.{ }^{t} \mathrm{Bu}\right) ;{ }^{13} \mathrm{CNMR}\left(125 \mathrm{MHz}, \mathrm{CDCl}_{3}\right) \delta$ 170.0, 167.0, 157.2, 152.0, 134.0, 133.2, 132.0, 129.8, 129.0, 127.8, $126.4,125.8,125.5,125.1,101.5,88.3,78.6,73.4,71.0,68.5,53.7,35.1,34.6,31.2,31.1,23.3$; HRMS (ESI) $\mathrm{m} / \mathrm{z}$ : found [M+Na] $]^{+} 640.2703, \mathrm{C}_{36} \mathrm{H}_{43} \mathrm{NO}_{6} \mathrm{~S}$ calcd for $[\mathrm{M}+\mathrm{Na}]^{+} 640.2703$.

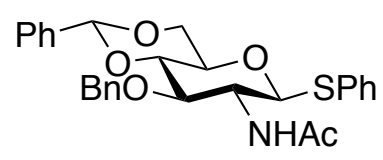

Phenyl

2-acetamido-3-O-benzyl-4,6-O-benzylidene-2-deoxy-1-thio- $\beta$-Dglucopyranoside (13a)

To a solution of compound $\mathbf{S O 9}^{\mathrm{SO2}}(321 \mathrm{mg}, 0.554 \mathrm{mmol})$ in EtOH $(5.50 \mathrm{~mL})$ was added ethylenediamine $(750 \mu \mathrm{L}, 11.1 \mathrm{mmol})$. After stirring for $17 \mathrm{~h}$ as the progress of the reaction was monitored by TLC (toluene/EtOAC $=3 / 1$ ), the reaction mixture was neutralized with $2 \mathrm{M} \mathrm{HCl}(\mathrm{pH}<8)$ and extracted with $\mathrm{CHCl}_{3}$. The organic layer was washed with $\mathrm{H}_{2} \mathrm{O}$ and brine, dried over $\mathrm{Na}_{2} \mathrm{SO}_{4}$, concentrated, and dried for $2 \mathrm{~h}$. The residue was dissolved in $\mathrm{CH}_{2} \mathrm{Cl}_{2}(5.50 \mathrm{~mL})$, followed by the addition of added $\mathrm{Ac}_{2} \mathrm{O}(78.7 \mu \mathrm{L}, 0.831 \mathrm{mmol})$. After stirring for $5.5 \mathrm{~h}$ at ambient temperature as the progress of the reaction was monitored by TLC (toluene/EtOAC $=3 / 1$ ), the reaction mixture was concentrated. The residue was crystallized (EtOH) to give compound 13a (221 mg, 81\%) as white 
crystals; mp >300 ${ }^{\circ} \mathrm{C} ;{ }^{1} \mathrm{H} \mathrm{NMR}\left(500 \mathrm{MHz}, \mathrm{CDCl}_{3}\right) \delta 7.50-7.28(\mathrm{~m}, 15 \mathrm{H}, 3 \mathrm{Ph}), 5.59(\mathrm{~s}, 1 \mathrm{H}, \mathrm{PhCH}<), 5.42$ $\left(\mathrm{d}, 1 \mathrm{H}, J_{2, \mathrm{NH}}=8.0 \mathrm{~Hz}, \mathrm{NH}-2\right), 5.28\left(\mathrm{~d}, 1 \mathrm{H}, J_{1,2}=10.0 \mathrm{~Hz}, \mathrm{H}-1\right), 4.88\left(\mathrm{~d}, 1 \mathrm{H}, J_{\text {gem }}=11.8 \mathrm{~Hz}, \mathrm{PhCH}\right.$ ), 4.63 (d, $1 \mathrm{H}, \mathrm{PhCH}$ ) $, 4.38\left(\mathrm{dd}, 1 \mathrm{H}, J_{5,6 a}=5.0 \mathrm{~Hz}, J_{\text {gem }}=10.4 \mathrm{~Hz}, \mathrm{H}-6 \mathrm{a}\right), 4.19\left(\mathrm{t}, 1 \mathrm{H}, J_{2,3}=J_{3,4}=9.3 \mathrm{~Hz}, \mathrm{H}-3\right.$ ), $3.80\left(\mathrm{t}, 1 \mathrm{H}, J_{5,6 b}=10.3 \mathrm{~Hz}, \mathrm{H}-6 \mathrm{~b}\right), 3.67\left(\mathrm{t}, 1 \mathrm{H}, J_{4,5}=9.3 \mathrm{~Hz}, \mathrm{H}-4\right), 3.58(\mathrm{~m}, 1 \mathrm{H}, \mathrm{H}-5$ ), 3.43 (near q, $1 \mathrm{H}$, $\mathrm{H}-2), 1.91(\mathrm{~s}, 3 \mathrm{H}, \mathrm{Ac})$.

* Compound is too insoluble to record specific rotation, ${ }^{13} \mathrm{C}$ NMR spectrum and HRMS.

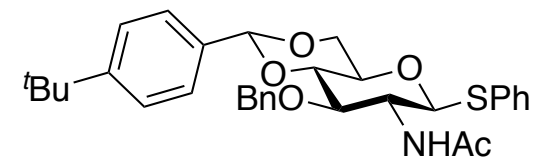

\section{Phenyl 2-acetamido-3-O-benzyl-4,6-O-p-tert-butylbenzylidene-2-deoxy-1-thio- $\beta$-D- glucopyranoside (13b)}

To a solution of compound $\mathbf{S 1 0}(310 \mathrm{mg}, 0.488 \mathrm{mmol})$ in $\mathrm{EtOH}(4.90 \mathrm{~mL})$ was added ethylenediamine (658 $\mu \mathrm{L}, 9.75 \mathrm{mmol}$ ). After stirring for $16 \mathrm{~h}$ as the progress of the reaction was monitored by TLC (toluene/EtOAC $=4 / 1)$, the reaction mixture was neutralized with $2 \mathrm{M} \mathrm{HCl}(\mathrm{pH}<8)$ and extracted with $\mathrm{CHCl}_{3}$. The organic layer was washed with $\mathrm{H}_{2} \mathrm{O}$ and brine, dried over $\mathrm{Na}_{2} \mathrm{SO}_{4}$, concentrated, and dried for $2 \mathrm{~h}$. The residue was dissolved in $\mathrm{CH}_{2} \mathrm{Cl}_{2}(4.90 \mathrm{~mL})$, followed by the addition of added $\mathrm{Ac}_{2} \mathrm{O}(69.2$ $\mu \mathrm{L}, 0.731 \mathrm{mmol}$ ). After stirring for $3 \mathrm{~h}$ at ambient temperature as the progress of the reaction was monitored by TLC (toluene/EtOAC $=4 / 1$ ), the reaction mixture was concentrated. The residue was crystallized ( $n$-hexane/EtOAc) to give compound $13 \mathrm{~b}$ ( $222 \mathrm{mg}, 83 \%$ ) as white crystals; mp $240-245^{\circ} \mathrm{C}$; $[\alpha]_{D}+11.8^{\circ}$ (c 1.0, $\left.\mathrm{CHCl}_{3}\right) ;{ }^{1} \mathrm{H}$ NMR $\left(500 \mathrm{MHz}, \mathrm{CDCl}_{3}\right)$ 8 7.47-7.27 (m, $\left.14 \mathrm{H}, 3 \mathrm{Ar}\right), 5.57(\mathrm{~s}, 1 \mathrm{H}, \mathrm{ArCH}<)$, $5.42\left(\mathrm{~d}, 1 \mathrm{H}, J_{2, \mathrm{NH}}=8.0 \mathrm{~Hz}, \mathrm{NH}-2\right), 5.26\left(\mathrm{~d}, 1 \mathrm{H}, J_{1,2}=10.5 \mathrm{~Hz}, \mathrm{H}-1\right), 4.90\left(\mathrm{~d}, 1 \mathrm{H}, J_{\mathrm{gem}}=12.0 \mathrm{~Hz}, \mathrm{PhCH}_{2}\right)$, $4.64\left(\mathrm{~d}, 1 \mathrm{H}, \mathrm{PhCH}\right.$ ) , 4.36 (dd, $\left.1 \mathrm{H}, J_{5,6 a}=5.0 \mathrm{~Hz}, J_{\mathrm{gem}}=10.4 \mathrm{~Hz}, \mathrm{H}-6 \mathrm{a}\right), 4.18\left(\mathrm{t}, 1 \mathrm{H}, J_{2,3}=J_{3,4}=9.3 \mathrm{~Hz}\right.$, $\mathrm{H}-3$ ), 3.79 (t, $\left.1 \mathrm{H}, J_{5,6 b}=10.3 \mathrm{~Hz}, \mathrm{H}-6 \mathrm{~b}\right), 3.67$ (t, $1 \mathrm{H}, \mathrm{J}_{4,5}=9.0 \mathrm{~Hz}, \mathrm{H}-4$ ), 3.57 (m, $\left.1 \mathrm{H}, \mathrm{H}-5\right), 3.43$ (near q, $1 \mathrm{H}, \mathrm{H}-2), 1.91(\mathrm{~s}, 3 \mathrm{H}, \mathrm{Ac}), 1.32\left(\mathrm{~s}, 9 \mathrm{H},{ }^{t} \mathrm{Bu}\right) ;{ }^{13} \mathrm{C} \mathrm{NMR}\left(125 \mathrm{MHz}, \mathrm{CDCl}_{3}\right) \delta 170.2,152.0,138.2,134.4$, $132.5,132.5,129.0,128.5,128.4,127.9,127.9,125.7,125.2,101.2$, 86.0, 82.5, 77.7, 74.6, 70.4, 68.7, 56.0, 34.7, 31.3, 23.6; HRMS (ESI) $\mathrm{m} / \mathrm{z}$ : found $[\mathrm{M}+\mathrm{Na}]^{+} 570.2285, \mathrm{C}_{32} \mathrm{H}_{37} \mathrm{NO}_{5} \mathrm{~S}$ calcd for $[\mathrm{M}+\mathrm{Na}]^{+}$ 570.2285 . 


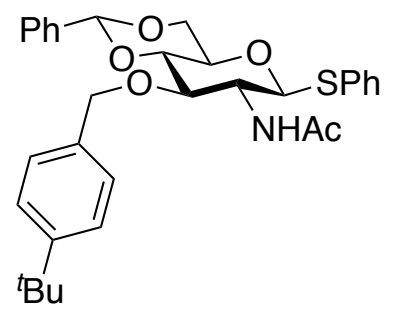

Phenyl 2-acetamido-4,6-O-benzylidene-3-O-p-tert-butylbenzyl-2-deoxy-1-thio- $\beta$-Dglucopyranoside (13c)

To a solution of compound $\mathbf{S 0 5}(300 \mathrm{mg}, 0.613 \mathrm{mmol})$ in DMF $(6.10 \mathrm{~mL})$ were added $p$-tertbutylbenzyl bromide ( $338 \mathrm{~mL}, 1.84 \mathrm{mmol}$ ), $\mathrm{NaH}$ ( $44.2 \mathrm{mg}, 1.84 \mathrm{mmol}$ ), and TBAI (22.6 mg, $61.3 \mu \mathrm{mol}$ ) at $0{ }^{\circ} \mathrm{C}$. After stirring for $30 \mathrm{~min}$ at $0{ }^{\circ} \mathrm{C}$ and for $8.5 \mathrm{~h}$ at ambient temperature as the progress of the reaction was monitored by TLC ( $n$-hexane/EtOAC $=2 / 1$ ), the reaction mixture was extracted with $\mathrm{CHCl}_{3}$ and washed with $\mathrm{H}_{2} \mathrm{O}$ and brine. The organic layer was dried over $\mathrm{Na}_{2} \mathrm{SO}_{4}$ and concentrated. The residue was purified by column chromatography ( $n$-hexane/EtOAc $=6 / 1$ ) and the resulting mixture was dried for $2 \mathrm{~h}$. The residue was dissolved in $\mathrm{EtOH}(6.10 \mathrm{~mL})$ and added ethylenediamine ( $831 \mu \mathrm{L}, 12.3 \mathrm{mmol}$ ). After stirring for $18 \mathrm{~h}$ under reflux as the progress of the reaction was monitored by TLC (toluene/EtOAC $=4 / 1)$, the reaction mixture was neutralized with $2 \mathrm{M} \mathrm{HCl}(\mathrm{pH}<8)$ and extracted with $\mathrm{CHCl}_{3}$. The organic layer was washed with $\mathrm{H}_{2} \mathrm{O}$ and brine, dried over $\mathrm{Na}_{2} \mathrm{SO}_{4}$, concentrated, and dried for $2 \mathrm{~h}$. The resulting residue was dissolved in $\mathrm{CH}_{2} \mathrm{Cl}_{2}(6.10 \mathrm{~mL})$ and added $\mathrm{Ac}_{2} \mathrm{O}(87.1 \mu \mathrm{L}, 0.920 \mathrm{mmol})$. After stirring for $2 \mathrm{~h}$ at ambient temperature as the progress of the reaction was monitored by TLC (toluene/EtOAC $=4 / 1$ ), the reaction mixture was co-evaporated with toluene. The residue was crystallized (EtOH) to give compound $13 \mathrm{c}(194 \mathrm{mg}, 64 \%)$ as white crystals; mp 290-293 ${ }^{\circ} \mathrm{C} ;[\alpha]_{\mathrm{D}}+139.0^{\circ}\left(\mathrm{c} 0.3, \mathrm{CHCl}_{3}\right) ;{ }^{1} \mathrm{H} \mathrm{NMR}\left(500 \mathrm{MHz}, \mathrm{CDCl}_{3}\right)$ 8 7.51-7.22(m, $\left.14 \mathrm{H}, 3 \mathrm{Ar}\right), 5.59$ (s, $1 \mathrm{H}, \mathrm{PhCH}<$ ), $5.40\left(\mathrm{~d}, 1 \mathrm{H}, J_{2, \mathrm{NH}}=8.0 \mathrm{~Hz}, \mathrm{NH}-2\right), 5.29\left(\mathrm{~d}, 1 \mathrm{H}, J_{1,2}=10.0 \mathrm{~Hz}, \mathrm{H}-1\right), 4.86$ and $4.59(2 \mathrm{~d}$, $\left.2 \mathrm{H}, J_{\text {gem }}=11.5 \mathrm{~Hz}, 2 \mathrm{ArCH}_{2}\right), 4.38\left(\mathrm{dd}, 1 \mathrm{H}, J_{5,6 \mathrm{a}}=5.0 \mathrm{~Hz}, J_{\text {gem }}=10.3 \mathrm{~Hz}, \mathrm{H}-6 \mathrm{a}\right), 4.20\left(\mathrm{t}, 1 \mathrm{H}, J_{2,3}=J_{3,4}=\right.$ $9.5 \mathrm{~Hz}, \mathrm{H}-3), 3.80\left(\mathrm{t}, 1 \mathrm{H}, J_{5,6 b}=10.0 \mathrm{~Hz}, \mathrm{H}-6 \mathrm{~b}\right), 3.66\left(\mathrm{t}, 1 \mathrm{H}, \mathrm{J}_{4,5}=9.0 \mathrm{~Hz}, \mathrm{H}-4\right), 3.59(\mathrm{~m}, 1 \mathrm{H}, \mathrm{H}-5), 3.40$ (near q, $1 \mathrm{H}, \mathrm{H}-2), 1.89(\mathrm{~s}, 3 \mathrm{H}, \mathrm{Ac}), 1.30\left(\mathrm{~s}, 9 \mathrm{H},{ }^{t} \mathrm{Bu}\right) ;{ }^{13} \mathrm{C} \mathrm{NMR}\left(125 \mathrm{MHz}, \mathrm{CDCl}_{3}\right) \delta 170.2,151.0,137.3$, $135.2,132.5,132.4,129.0,128.3,128.3,127.9,126.0,125.4,101.2,86.0,82.6,77.5,74.5,70.3,68.7$, $56.1,34.6,31.3,30.3,23.6$; HRMS (ESI) $\mathrm{m} / \mathrm{z}$ : found $[\mathrm{M}+\mathrm{Na}]^{+} 570.2285, \mathrm{C}_{32} \mathrm{H}_{37} \mathrm{NO}_{5} \mathrm{~S}$ calcd for $[\mathrm{M}+\mathrm{Na}]^{+}$ 570.2284 . 
<smiles>CC(C)(N)C1OCCC(c2ccc(Br)cc2)OC1COCc1ccc(Br)cc1</smiles>

Phenyl 2-acetamido-3-0-p-tert-butylbenzyl-4,6-0-p-tert-butylbenzylidene-2-deoxy-1thio- $\beta$-D-glucopyranoside (13d)

To a solution of compound $\mathbf{S 1 1}(244 \mathrm{mg}, 0.353 \mathrm{mmol})$ in EtOH $(3.50 \mathrm{~mL})$ was added ethylenediamine (476 $\mu \mathrm{L}, 7.05 \mathrm{mmol})$. After stirring for $24 \mathrm{~h}$ as the progress of the reaction was monitored by TLC (toluene/EtOAc $=4 / 1)$, the reaction mixture was neutralized with $2 \mathrm{M} \mathrm{HCl}(\mathrm{pH}<8)$ and extracted with $\mathrm{CHCl}_{3}$. The organic layer was washed with $\mathrm{H}_{2} \mathrm{O}$ and brine, dried over $\mathrm{Na}_{2} \mathrm{SO}_{4}$, concentrated, and dried for $2 \mathrm{~h}$. The residue was dissolved in $\mathrm{CH}_{2} \mathrm{Cl}_{2}(3.50 \mathrm{~mL})$, followed by the addition of $\mathrm{Ac}_{2} \mathrm{O}(50.1 \mu \mathrm{L}$, $0.529 \mathrm{mmol}$ ). After stirring for $2 \mathrm{~h}$ at ambient temperature as the progress of the reaction was monitored by TLC (toluene/EtOAC $=4 / 1$ ), the reaction mixture was concentrated. The residue was crystallized ( $n$-hexane/EtOAc) to give compound $13 \mathrm{~d}$ (176 mg, 83\%) as white crystals; mp 231-235 ${ }^{\circ} \mathrm{C}$; $[\alpha]_{D}+10.3^{\circ}\left(\mathrm{c} 1.0, \mathrm{CHCl}_{3}\right) ;{ }^{1} \mathrm{H}$ NMR $\left(500 \mathrm{MHz}, \mathrm{CDCl}_{3}\right)$ 8 7.47-7.22 (m, $\left.13 \mathrm{H}, 3 \mathrm{Ar}\right), 5.57(\mathrm{~s}, 1 \mathrm{H}, \mathrm{ArCH}<)$, $5.40\left(\mathrm{~d}, 1 \mathrm{H}, J_{2, N H}=8.0 \mathrm{~Hz}, \mathrm{NH}-2\right), 5.28\left(\mathrm{~d}, 1 \mathrm{H}, J_{1,2}=10.5 \mathrm{~Hz}, \mathrm{H}-1\right), 4.87\left(\mathrm{~d}, 1 \mathrm{H}, J_{\text {gem }}=11.5 \mathrm{~Hz}, \mathrm{ArCH}_{2}\right)$, $4.59\left(\mathrm{~d}, 1 \mathrm{H}, \mathrm{ArCH}_{2}\right), 4.36\left(\mathrm{dd}, 1 \mathrm{H}, J_{5,6 \mathrm{a}}=5.0 \mathrm{~Hz}, J_{\mathrm{gem}}=10.4 \mathrm{~Hz}, \mathrm{H}-6 \mathrm{a}\right), 4.19\left(\mathrm{t}, 1 \mathrm{H}, J_{2,3}=J_{3,4}=9.3 \mathrm{~Hz}\right.$, $\mathrm{H}-3$ ), 3.79 (t, $1 \mathrm{H}, J_{5,6 b}=10.3 \mathrm{~Hz}, \mathrm{H}-6 \mathrm{~b}$ ), 3.65 (t, $\left.1 \mathrm{H}, J_{4,5}=9.3 \mathrm{~Hz}, \mathrm{H}-4\right), 3.57(\mathrm{~m}, 1 \mathrm{H}, \mathrm{H}-5$ ), 3.40 (near $\mathrm{q}, 1 \mathrm{H}, \mathrm{H}-2), 1.89(\mathrm{~s}, 3 \mathrm{H}, \mathrm{Ac}), 1.32\left(\mathrm{~s}, 9 \mathrm{H},{ }^{\mathrm{t}} \mathrm{Bu}\right), 1.30\left(\mathrm{~s}, 9 \mathrm{H},{ }^{\mathrm{t}} \mathrm{Bu}\right) ;{ }^{13} \mathrm{C} \mathrm{NMR}\left(125 \mathrm{MHz}, \mathrm{CDCl}_{3}\right) \delta 170.2$, 152.0, 150.9, 135.2, 134.5, 132.5, 132.4, 129.0, 128.3, 127.9, 125.7, 125.4, 125.2, 101.2, 86.0, 82.6, 77.5, 74.5, 70.4, 68.7, 56.0, 34.7, 34.6, 31.3, 31.3, 30.3, 23.6; HRMS (ESI) $\mathrm{m} / \mathrm{z}$ : found $[\mathrm{M}+\mathrm{Na}]^{+}$ $626.2911, \mathrm{C}_{36} \mathrm{H}_{45} \mathrm{NO}_{5} \mathrm{~S}$ calcd for $[\mathrm{M}+\mathrm{Na}]^{+} 626.2911$.

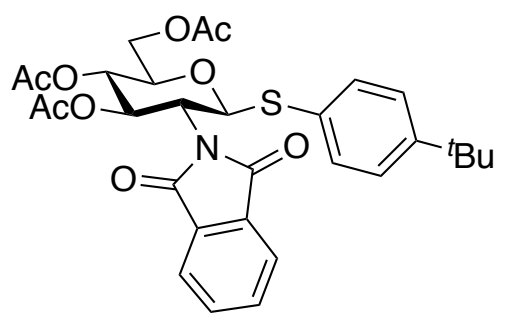

p-tert-Butylphenyl

3,4,6-tri-O-acetyl-2-deoxy-2-phthalimido-1-thio- $\beta$-Dglucopyranoside ( $\mathrm{SO3}$ )

To a solution of compound $\mathrm{SO1}^{\mathrm{S04}}(10.0 \mathrm{~g}, 20.9 \mathrm{mmol})$ in $\mathrm{CH}_{2} \mathrm{Cl}_{2}(209 \mathrm{~mL})$ were added p-tertbutylbenzenethiol $(4.22 \mathrm{~mL}, 25.1 \mathrm{mmol})$ and $\mathrm{BF}_{3} \cdot \mathrm{OEt}_{2}(3.94 \mathrm{~mL}, 31.4 \mathrm{mmol})$. After stirring for $40 \mathrm{~h}$ at 
ambient temperature as the progress of the reaction was monitored by TLC ( $n$-hexane/EtOAc $=3 / 2)$, the reaction was quenched by adding crashed ice. The reaction mixture was extracted with $\mathrm{CHCl}_{3}$ and washed with $\mathrm{H}_{2} \mathrm{O}$, satd. aq. $\mathrm{NaHCO}_{3}$ and brine. The organic layer was dried over $\mathrm{Na}_{2} \mathrm{SO}_{4}$ and concentrated. The residue was purified by silica gel column chromatography ( $n$-hexane/EtOAc $=2 / 1$ ) to give compound $\mathbf{S O 3}\left(11.4 \mathrm{~g}, 94 \%\right.$ ) as a white foam; $[\alpha]_{\mathrm{D}}+44.7^{\circ}$ (c 1.0, $\mathrm{CHCl}_{3}$ ); ${ }^{1} \mathrm{H} \mathrm{NMR}(500 \mathrm{MHz}$, $\left.\mathrm{CDCl}_{3}\right) \delta 7.88-7.27\left(\mathrm{~m}, 8 \mathrm{H}, 2 \mathrm{Ar}\right.$ ), $5.80\left(\mathrm{dd}, 1 \mathrm{H}, J_{3,4}=9.5 \mathrm{~Hz}, J_{2,3}=10.3 \mathrm{~Hz}, \mathrm{H}-3\right), 5.67\left(\mathrm{~d}, 1 \mathrm{H}, J_{1,2}=\right.$ $10.5 \mathrm{~Hz}, \mathrm{H}-1), 5.15$ (dd, $\left.1 \mathrm{H}, J_{4,5}=10.0 \mathrm{~Hz}, \mathrm{H}-4\right), 4.36(\mathrm{t}, 1 \mathrm{H}, \mathrm{H}-2), 4.29\left(\mathrm{dd}, 1 \mathrm{H}, J_{5,6 a}=5.3 \mathrm{~Hz}, J_{\text {gem }}=\right.$ 12.3 Hz, H-6a), 4.22 (dd, 1 H, J5,6b = 2.3 Hz, H-6b), 3.90 (m, $1 \mathrm{H}, \mathrm{H}-5), 2.10$ (s, $3 \mathrm{H}, \mathrm{Ac}), 2.02(\mathrm{~s}, 3 \mathrm{H}, \mathrm{Ac}$ ), 1.85 (s, $3 \mathrm{H}, \mathrm{Ac}), 1.30$ (s, $\left.9 \mathrm{H},{ }^{t} \mathrm{Bu}\right) ;{ }^{13} \mathrm{C}$ NMR (125 MHz, CDCl $) \delta 170.7,170.1,169.5,167.8,167.0$, $151.8,134.5,134.3,133.5,131.6,131.2,127.2$, 126.0, 123.7, 83.2, 75.8, 71.7, 68.7, 62.2, 53.7, 34.6, 31.2, 20.8, 20.6, 20.4; HRMS (ESI) $\mathrm{m} / \mathrm{z}$ : found $[\mathrm{M}+\mathrm{Na}]^{+} 606.1768, \mathrm{C}_{30} \mathrm{H}_{33} \mathrm{NO}_{9} \mathrm{~S}$ calcd for $[\mathrm{M}+\mathrm{Na}]^{+}$ 606.1768 .

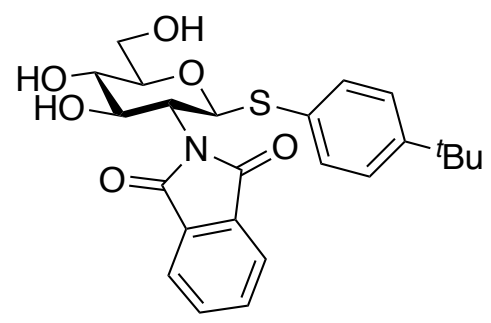

\section{p-tert-Butylphenyl 2-deoxy-2-phthalimido-1-thio- $\beta$-D-glucopyranoside (S04)}

To a solution of compound $\mathbf{S 0 3}$ (11.4 g, $19.5 \mathrm{mmol})$ in $\mathrm{MeOH}(195 \mathrm{~mL}$ ) was added $28 \mathrm{wt} \% \mathrm{NaOMe}$ (376 $\mathrm{mg}, 1.95 \mathrm{mmol}$ ). After stirring for $2 \mathrm{~h}$ at ambient temperature as the progress of the reaction was monitored by $\operatorname{TLC}\left(\mathrm{CHCl}_{3} / \mathrm{MeOH}=15 / 1\right)$, the reaction mixture was neutralized with Muromac $\left(\mathrm{H}^{+}\right)$and filtered through cotton, followed by washing with $\mathrm{MeOH}$. The combined filtrate and washings were concentrated. The residue was purified by silica gel column chromatography $\left(\mathrm{CHCl}_{3} / \mathrm{MeOH}=40 / 1\right)$ to give compound S04 $(8.83 \mathrm{~g}, 99 \%)$ as a white foam; $[\alpha]_{D}+77.8^{\circ}(\mathrm{c} 1.0, \mathrm{MeOH})$; ${ }^{1} \mathrm{H}$ NMR $\left(500 \mathrm{MHz}, \mathrm{CD}_{3} \mathrm{OD}\right) \delta$ 7.91-7.26 (m, $\left.8 \mathrm{H}, 2 \mathrm{Ar}\right), 5.53\left(\mathrm{~d}, 1 \mathrm{H}, \mathrm{J}_{1,2}=10.4 \mathrm{~Hz}, \mathrm{H}-1\right), 4.23$ (dd, $1 \mathrm{H}$, $\left.J_{3,4}=8.0 \mathrm{~Hz}, J_{2,3}=10.2 \mathrm{~Hz}, \mathrm{H}-3\right), 4.08(\mathrm{t}, 1 \mathrm{H}, \mathrm{H}-2), 3.93\left(\mathrm{dd}, 1 \mathrm{H}, J_{5,6 \mathrm{a}}=2.0 \mathrm{~Hz}, J_{\text {gem }}=12.0 \mathrm{~Hz}, \mathrm{H}-6 \mathrm{a}\right)$, $3.75\left(\mathrm{dd}, 1 \mathrm{H}, \mathrm{J}_{5,6 \mathrm{~b}}=5.5 \mathrm{~Hz}, \mathrm{H}-6 \mathrm{~b}\right), 3.48-3.40(\mathrm{~m}, 2 \mathrm{H}, \mathrm{H}-4, \mathrm{H}-5), 1.27\left(\mathrm{~s}, 9 \mathrm{H},{ }^{\mathrm{t}} \mathrm{Bu}\right) ;{ }^{13} \mathrm{C}$ NMR $(125 \mathrm{MHz}$, $\left.\mathrm{CD}_{3} \mathrm{OD}\right) \delta 169.8,169.3,152.2,135.7,135.6,133.2,133.1,133.0,130.8,127.0,124.5,124.2,85.7,82.7$, 73.9, 72.3, 62.9, 58.0, 35.4, 31.7; HRMS (ESI) $\mathrm{m} / \mathrm{z}$ : found $[\mathrm{M}+\mathrm{Na}]^{+} 480.1451, \mathrm{C}_{24} \mathrm{H}_{27} \mathrm{NO}_{6} \mathrm{~S}$ calcd for $[\mathrm{M}+\mathrm{Na}]^{+} 480.1451$. 


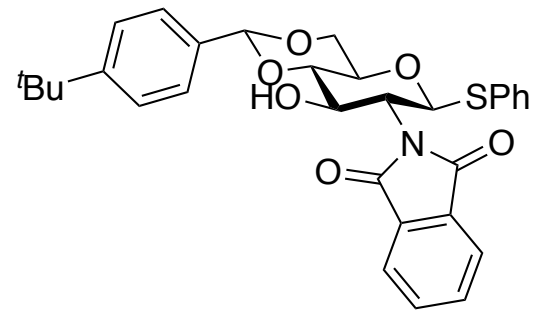

Phenyl

4,6-O-p-tert-butylbenzylidene-2-deoxy-2-phthalimido-1-thio- $\beta$-Dglucopyranoside (S06)

To a solution of compound S02 $(4.34 \mathrm{~g}, 10.8 \mathrm{mmol})$ in MeCN/THF = 2/1 (108 mL) were added compound 2c (4.50 g, $21.6 \mathrm{mmol})$ and ( \pm )-10-camphorsulfonic acid (251 mg, $1.08 \mathrm{mmol})$. After stirring for $26.5 \mathrm{~h}$ at ambient temperature as the progress of the reaction was monitored by TLC ( $n$ hexane/EtOAc $=7 / 3$ ), the reaction was quenched with $\mathrm{Et}_{3} \mathrm{~N}$ and concentrated. The residue was purified by silica gel column chromatography ( $n$-hexane/EtOAc $=3 / 1)$ to give compound S06 $(5.19 \mathrm{~g}$, $88 \%$ ) as a white foam; $[\alpha]_{\mathrm{D}}+31.2^{\circ}$ (c 1.0, $\left.\mathrm{CHCl}_{3}\right) ;{ }^{1} \mathrm{H}$ NMR (500 MHz, $\left.\mathrm{CDCl}_{3}\right) \delta 7.91-7.26(\mathrm{~m}, 13 \mathrm{H}, 3$ Ar), $5.70\left(\mathrm{~d}, 1 \mathrm{H}, J_{1,2}=10.5 \mathrm{~Hz}, \mathrm{H}-1\right), 5.55\left(\mathrm{~s}, 1 \mathrm{H}, \mathrm{ArCH}<\right.$ ), $4.64\left(\mathrm{dt}, 1 \mathrm{H}, J_{3, \mathrm{OH}}=3.3 \mathrm{~Hz}, J_{2,3}=J_{3,4}=9.7\right.$ $\mathrm{Hz}, \mathrm{H}-3$ ), 4.39 (dd, $\left.1 \mathrm{H}, \mathrm{J}_{5,6 \mathrm{a}}=5.0 \mathrm{~Hz}, \mathrm{~J}_{\mathrm{gem}}=10.0 \mathrm{~Hz}, \mathrm{H}-6 \mathrm{a}\right), 4.34(\mathrm{t}, 1 \mathrm{H}, \mathrm{H}-2), 3.82\left(\mathrm{t}, 1 \mathrm{H}, \mathrm{J}_{5,6 \mathrm{~b}}=10.0\right.$ $\mathrm{Hz}, \mathrm{H}-6 b), 3.71(\mathrm{~m}, 1 \mathrm{H}, \mathrm{H}-5), 3.60\left(\mathrm{t}, 1 \mathrm{H}, \mathrm{J}_{4,5}=9.3 \mathrm{~Hz}, \mathrm{H}-4\right), 2.49(\mathrm{~d}, 1 \mathrm{H}, \mathrm{OH}-3), 1.30\left(\mathrm{~s}, 9 \mathrm{H},{ }^{t} \mathrm{Bu}\right) ;{ }^{13} \mathrm{C}$ NMR $\left(125 \mathrm{MHz}, \mathrm{CDCl}_{3}\right) \delta 168.2,167.5,152.5,134.2,134.0,132.7,131.7,131.6,129.0,128.1,126.0$, $125.4,123.9,123.4,102.1,84.3,81.9,70.3,69.8,68.6,55.4,34.7,31.3$; HRMS (ESI) $m / z$ : found $[\mathrm{M}+\mathrm{Na}]^{+} 568.1764, \mathrm{C}_{31} \mathrm{H}_{31} \mathrm{NO}_{6} \mathrm{~S}$ calcd for $[\mathrm{M}+\mathrm{Na}]^{+} 568.1764$.

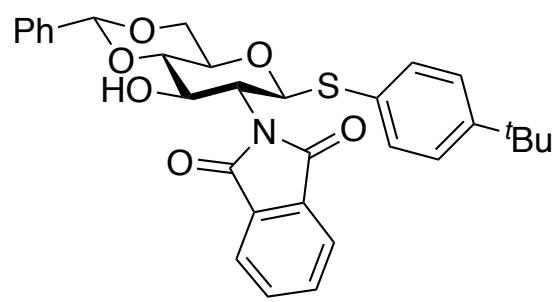

p-tert-Butylphenyl

4,6-O-benzylidene-2-deoxy-2-phthalimido-1-thio- $\beta$-Dglucopyranoside (S07)

To a solution of compound S04 $(4.38 \mathrm{~g}, 9.57 \mathrm{mmol})$ in MeCN/THF $=2 / 1(96.0 \mathrm{~mL})$ were added benzaldehyde dimethyl acetal $(2.88 \mathrm{~mL}, 19.1 \mathrm{mmol})$ and ( \pm )-10-camphorsulfonic acid ( $222 \mathrm{mg}, 0.957$ $\mathrm{mmol})$. After stirring for $19 \mathrm{~h}$ at ambient temperature as the progress of the reaction was monitored by TLC $(n$-hexane/EtOAc $=7 / 3)$, the reaction was quenched with $\mathrm{Et}_{3} \mathrm{~N}$ and concentrated. The residue was purified by silica gel column chromatography ( $n$-hexane/EtOAc $=3 / 1$ ) to give compound $\mathbf{S 0 7}$ (4.87 g, 93\%) as a white foam; $[\alpha]_{D}+38.2^{\circ}$ (c 1.0, $\left.\mathrm{CHCl}_{3}\right) ;{ }^{1} \mathrm{H} \mathrm{NMR}\left(500 \mathrm{MHz}, \mathrm{CDCl}_{3}\right) \delta 7.92-7.26(\mathrm{~m}$, $13 \mathrm{H}, 3 \mathrm{Ar}), 5.65\left(\mathrm{~d}, 1 \mathrm{H}, J_{1,2}=10.5 \mathrm{~Hz}, \mathrm{H}-1\right), 5.57(\mathrm{~s}, 1 \mathrm{H}, \mathrm{PhCH}<), 4.64(\mathrm{~m}, 1 \mathrm{H}, \mathrm{H}-3), 4.41$ (dd, $1 \mathrm{H}, J_{5,6 a}$ 
$\left.=4.8 \mathrm{~Hz}, J_{\text {gem }}=10.3 \mathrm{~Hz}, \mathrm{H}-6 \mathrm{a}\right), 4.34\left(\mathrm{t}, 1 \mathrm{H}, J_{2,3}=10.3 \mathrm{~Hz}, \mathrm{H}-2\right), 3.83\left(\mathrm{t}, 1 \mathrm{H}, J_{5,6 \mathrm{~b}}=10.3 \mathrm{~Hz}, \mathrm{H}-6 \mathrm{~b}\right), 3.71$ $(\mathrm{m}, 1 \mathrm{H}, \mathrm{H}-5), 3.61\left(\mathrm{t}, 1 \mathrm{H}, J_{3,4}=J_{4,5}=9.3 \mathrm{~Hz}, \mathrm{H}-4\right), 2.51\left(\mathrm{~d}, 1 \mathrm{H}, J_{3, \mathrm{OH}}=3.5 \mathrm{~Hz}, \mathrm{OH}-3\right), 1.29\left(\mathrm{~s}, 9 \mathrm{H},{ }^{t} \mathrm{Bu}\right)$; ${ }^{13} \mathrm{C}$ NMR $\left(125 \mathrm{MHz}, \mathrm{CDCl}_{3}\right) \delta 168.2,167.6,151.5,136.9,132.8,131.7,129.4,128.4,128.1,126.3$, 126.0, 123.9, 123.4, 102.0, 84.5, 81.9, 70.2, 69.8, 69.7, 68.6, 55.6, 55.6, 34.6, 31.2; HRMS (ESI) m/z: found $[\mathrm{M}+\mathrm{Na}]^{+} 568.1764, \mathrm{C}_{31} \mathrm{H}_{31} \mathrm{NO}_{6} \mathrm{~S}$ calcd for $[\mathrm{M}+\mathrm{Na}]^{+} 568.1764$.

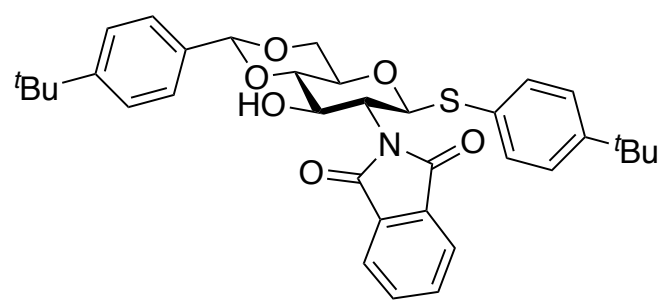

p-tert-Butylphenyl 2-acetamido-3-O-acetyl-4,6-O-p-tert-butylbenzylidene-2-deoxy-1thio- $\beta$-D-glucopyranoside (S08)

To a solution of compound S04 (4.45 g, $9.73 \mathrm{mmol}$ ) in MeCN/THF = 2/1 (97.0 mL) were added compound 2c (4.06 g, $19.5 \mathrm{mmol})$ and ( \pm )-10-camphorsulfonic acid (226 mg, $0.973 \mathrm{mmol})$. After stirring for $19 \mathrm{~h}$ at ambient temperature as the progress of the reaction was monitored by TLC ( $n$ hexane/EtOAc $=7 / 3$ ), the reaction was quenched with $\mathrm{Et}_{3} \mathrm{~N}$ and concentrated. The residue was purified by silica gel column chromatography ( $n$-hexane/EtOAc $=3 / 1)$ to give compound S08 $(5.28 \mathrm{~g}$, $90 \%$ ) as a white foam; $[\alpha]_{D}+38.5^{\circ}$ (c 1.0, $\left.\mathrm{CHCl}_{3}\right) ;{ }^{1} \mathrm{H} \mathrm{NMR}\left(500 \mathrm{MHz}, \mathrm{CDCl}_{3}\right) \delta 7.92-7.26(\mathrm{~m}, 12 \mathrm{H}, 3$ $\operatorname{Ar}), 5.66\left(\mathrm{~d}, 1 \mathrm{H}, J_{1,2}=10.5 \mathrm{~Hz}, \mathrm{H}-1\right), 5.55(\mathrm{~s}, 1 \mathrm{H}, \operatorname{ArCH}<), 4.64\left(\mathrm{dt}, 1 \mathrm{H}, J_{3, \mathrm{OH}}=2.2 \mathrm{~Hz}, J_{2,3}=J_{3,4}=9.5\right.$ $\mathrm{Hz}, \mathrm{H}-3), 4.40\left(\mathrm{dd}, 1 \mathrm{H}, J_{5,6 \mathrm{a}}=5.0 \mathrm{~Hz}, J_{\text {gem }}=10.5 \mathrm{~Hz}, \mathrm{H}-6 \mathrm{a}\right), 4.34(\mathrm{t}, 1 \mathrm{H}, \mathrm{H}-2), 3.82\left(\mathrm{t}, 1 \mathrm{H}, J_{5,6 b}=10.3\right.$ $\mathrm{Hz}, \mathrm{H}-6 b), 3.71(\mathrm{~m}, 1 \mathrm{H}, \mathrm{H}-5), 3.61$ (t, $1 \mathrm{H}, \mathrm{H}-4), 2.47$ (d, $1 \mathrm{H}, \mathrm{OH}-3), 1.30\left(\mathrm{~s}, 9 \mathrm{H},{ }^{t} \mathrm{Bu}\right), 1.29\left(\mathrm{~s}, 9 \mathrm{H},{ }^{t} \mathrm{Bu}\right)$; ${ }^{13} \mathrm{C}$ NMR $\left(125 \mathrm{MHz} \mathrm{CDCl}_{3}\right) \delta 168.2,167.6,152.5,151.5,134.2,134.0,132.8,131.6,128.1,126.0$, 126.0, 125.4, 123.9, 123.4, 102.0, 84.5, 81.9, 70.3, 69.8, 68.6, 55.6, 34.7, 34.6, 31.3, 31.2; HRMS (ESI) $\mathrm{m} / \mathrm{z}$ : found $[\mathrm{M}+\mathrm{Na}]^{+} 624.2390, \mathrm{C}_{35} \mathrm{H}_{39} \mathrm{NO}_{6} \mathrm{~S}$ calcd for $[\mathrm{M}+\mathrm{Na}]^{+} 624.2390$.

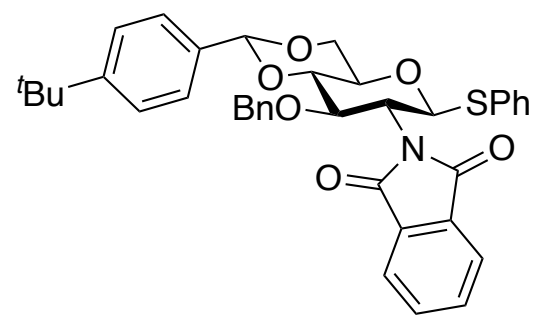

Phenyl 3-O-benzyl-4,6-O-p-tert-butylbenzylidene-2-deoxy-2-phthalimido-1-thio- $\beta$-Dglucopyranoside (S10) 
To a solution of compound S06 (301 mg, $0.552 \mathrm{mmol})$ in DMF $(5.50 \mathrm{~mL})$ were added $\mathrm{BnBr}(191 \mu \mathrm{L}$, $1.65 \mathrm{mmol}), \mathrm{NaH}(39.6 \mathrm{mg}, 1.65 \mathrm{mmol})$ and TBAl $(20.4 \mathrm{mg}, 55.2 \mu \mathrm{mol})$ at $0{ }^{\circ} \mathrm{C}$. After stirring for 30 min at $0{ }^{\circ} \mathrm{C}$ and for $23 \mathrm{~h}$ at ambient temperature as the progress of the reaction was monitored by $\operatorname{TLC}$ ( $n$-hexane/EtOAC $=2 / 1$ ), the reaction mixture was extracted with $\mathrm{CHCl}_{3}$. The organic layer was washed with $\mathrm{H}_{2} \mathrm{O}$ and brine, dried over $\mathrm{Na}_{2} \mathrm{SO}_{4}$, and concentrated. The residue was purified by silica gel column chromatography ( $n$-hexane/EtOAc $=5 / 1$ ) to give compound $\mathbf{S 1 0}(326 \mathrm{mg}, 93 \%$ ) as a white amorphous solid; $[\alpha]_{\mathrm{D}}+95.2^{\circ}$ (c 1.0, $\left.\mathrm{CHCl}_{3}\right) ;{ }^{1} \mathrm{H} \mathrm{NMR}\left(500 \mathrm{MHz}, \mathrm{CDCl}_{3}\right) \delta$ 7.87-6.85 (m, $18 \mathrm{H}, 4 \mathrm{Ar}$ ), $5.61\left(\mathrm{~d}, 1 \mathrm{H}, \mathrm{J}_{1,2}=10.4 \mathrm{~Hz}, \mathrm{H}-1\right), 5.61(\mathrm{~s}, 1 \mathrm{H}, \mathrm{ArCH}<), 4.78\left(\mathrm{~d}, 1 \mathrm{H}, \mathrm{J}_{\text {gem }}=12.5 \mathrm{~Hz}, \mathrm{PhCH}_{2}\right), 4.50(\mathrm{~d}, 1 \mathrm{H}$, $\mathrm{PhCH}_{2}$ ), 4.44-4.39 (m, $\left.2 \mathrm{H}, \mathrm{H}-3, \mathrm{H}-6 \mathrm{a}\right), 4.28$ (t, $1 \mathrm{H}, J_{2,3}=10.3 \mathrm{~Hz}, \mathrm{H}-2$ ), 3.84 (t, $1 \mathrm{H}, J_{3,4}=J_{4,5}=10.3 \mathrm{~Hz}$, $\mathrm{H}-4), 3.79\left(\mathrm{t}, 1 \mathrm{H}, J_{5,6 b}=J_{\text {gem }}=9.3 \mathrm{~Hz}, \mathrm{H}-6 \mathrm{~b}\right), 3.70(\mathrm{~m}, 1 \mathrm{H}, \mathrm{H}-5), 1.32\left(\mathrm{~s}, 9 \mathrm{H},{ }^{\mathrm{t}} \mathrm{Bu}\right) ;{ }^{13} \mathrm{C} \mathrm{NMR}(125 \mathrm{MHz}$, $\left.\mathrm{CDCl}_{3}\right) \delta 167.8,167.2,152.1,137.8,134.4,134.0,133.8,132.8,131.7,131.6,128.9,128.2,128.1$, 128.0, 127.4, 125.7, 125.3, 123.5, 123.4, 101.3, 84.1, 82.8, 75.5, 74.2, 70.4, 68.7, 54.7, 34.7, 31.3; HRMS (ESI) $m / z$ : found $[\mathrm{M}+\mathrm{Na}]^{+} 658.2230, \mathrm{C}_{38} \mathrm{H}_{37} \mathrm{NO}_{6} \mathrm{~S}$ calcd for $[\mathrm{M}+\mathrm{Na}]^{+} 658.2234$.

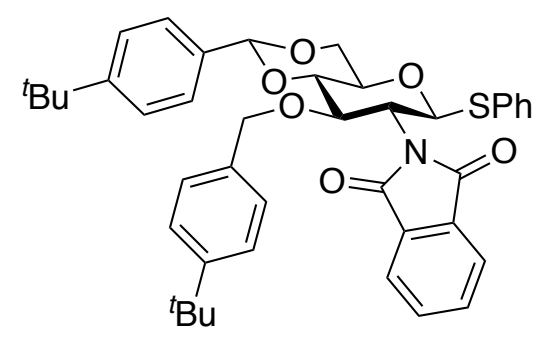

Phenyl 3-O-p-tert-butylbenzyl-4,6-O-p-tert-butylbenzylidene-2-deoxy-2-phthalimido1-thio- $\beta$-D-glucopyranoside (S11)

To a solution of compound S06 (301 mg, $0.552 \mathrm{mmol}$ ) in DMF $(5.50 \mathrm{~mL})$ were added p-tertbutylbenzyl bromide (303 $\mu \mathrm{L}, 1.65 \mathrm{mmol}$ ), $\mathrm{NaH}(39.6 \mathrm{mg}, 1.65 \mathrm{mmol}$ ), and TBAI (20.4 mg, $55.2 \mu \mathrm{mol}$ ) at $0{ }^{\circ} \mathrm{C}$. After stirring for $30 \mathrm{~min}$ at $0{ }^{\circ} \mathrm{C}$ and for $24 \mathrm{~h}$ at ambient temperature as the progress of the reaction was monitored by TLC ( $n$-hexane/EtOAC $=2 / 1)$, the reaction mixture was extracted with $\mathrm{CHCl}_{3}$. The organic layer was washed with $\mathrm{H}_{2} \mathrm{O}$ and brine, dried over $\mathrm{Na}_{2} \mathrm{SO}_{4}$, and concentrated. The residue was purified by silica gel column chromatography ( $n$-hexane/EtOAc $=6 / 1)$ and crystallized $(n$ hexane/EtOAc) to give compound S11 (261 mg, 68\%) as white crystals; mp 190-194 ${ }^{\circ} \mathrm{C} ;[\alpha]_{\mathrm{D}}+76.5^{\circ}$ (c 1.0, $\mathrm{CHCl}_{3}$ ); ${ }^{1} \mathrm{H}$ NMR (500 MHz, $\mathrm{CDCl}_{3}$ ) $\delta 7.92-6.91$ (m, $17 \mathrm{H}, 4 \mathrm{Ar}$ ), $5.63\left(\mathrm{~d}, 1 \mathrm{H}, \mathrm{J}_{1,2}=10.5 \mathrm{~Hz}, \mathrm{H}-1\right.$ ), $5.60(\mathrm{~s}, 1 \mathrm{H}, \mathrm{ArCH}<), 4.76\left(\mathrm{~d}, 1 \mathrm{H}, J_{\mathrm{gem}}=12.0 \mathrm{~Hz}, \mathrm{ArCH}_{2}\right), 4.52\left(\mathrm{~d}, 1 \mathrm{H}, \mathrm{ArCH}_{2}\right), 4.44\left(\mathrm{t}, 1 \mathrm{H}, J_{2,3}=J_{3,4}=\right.$ $9.7 \mathrm{~Hz}, \mathrm{H}-3), 4.40\left(\mathrm{dd}, 1 \mathrm{H}, J_{5,6 a}=5.3 \mathrm{~Hz}, J_{\mathrm{gem}}=10.6 \mathrm{~Hz}, \mathrm{H}-6 \mathrm{a}\right), 4.32(\mathrm{t}, 1 \mathrm{H}, \mathrm{H}-2), 3.84\left(\mathrm{t}, 1 \mathrm{H}, J_{5,6 b}=\right.$ $10.3 \mathrm{~Hz}, \mathrm{H}-6 \mathrm{~b}), 3.80\left(\mathrm{t}, 1 \mathrm{H}, \mathrm{J}_{4,5}=9.3 \mathrm{~Hz}, \mathrm{H}-4\right), 3.70(\mathrm{~m}, 1 \mathrm{H}, \mathrm{H}-5), 1.32\left(\mathrm{~s}, 9 \mathrm{H},{ }^{t} \mathrm{Bu}\right), 1.19\left(\mathrm{~s}, 9 \mathrm{H},{ }^{t} \mathrm{Bu}\right)$; ${ }^{13} \mathrm{C}$ NMR $\left(125 \mathrm{MHz}, \mathrm{CDCl}_{3}\right) \delta 167.8,167.3,152.0,150.4,134.8,134.4,134.1,134.0,132.7,131.7$, 
128.9, 128.1, 127.8, 125.7, 125.3, 125.0, 123.7, 123.3, 101.3, 84.1, 82.9, 75.2, 73.8, 70.4, 68.6, 54.8, 34.7, 34.3, 31.3, 31.3; HRMS (ESI) $\mathrm{m} / \mathrm{z}$ : found $[\mathrm{M}+\mathrm{Na}]^{+} 714.2860, \mathrm{C}_{42} \mathrm{H}_{45} \mathrm{NO}_{6} \mathrm{~S}$ calcd for $[\mathrm{M}+\mathrm{Na}]^{+}$ 714.2860.

\subsection{TBBz-protected GlcCer}
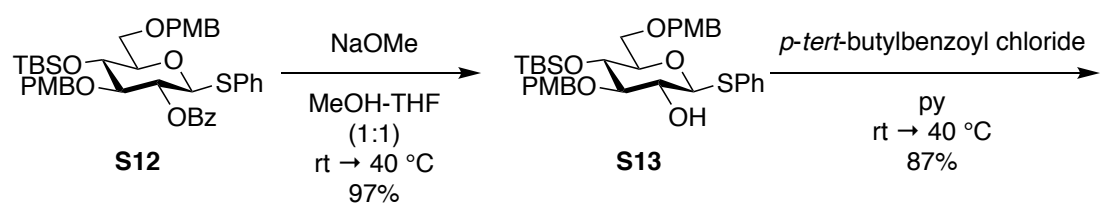

Supporting Scheme 8. Synthesis of Glc donor $\mathbf{1 4 b}$

Supporting Scheme 8. Synthesis of Glc donor $14 \mathrm{~b}$

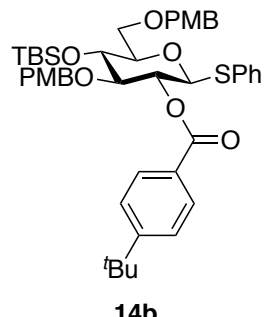

$14 b$

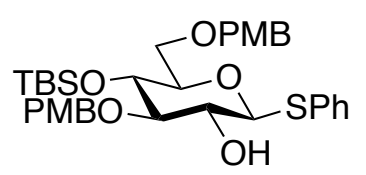

Phenyl 4-O-tert-butyldimethylsilyl-3,6-di-O-p-methoxybenzyl-1-thio- $\beta$-Dglucopyranoside (S13)

To a solution of compound $\mathbf{S 1 2} \mathbf{2}^{\mathrm{S} 05}$ (406 mg, $0.555 \mathrm{mmol}$ ) in MeOH/THF = 1/1 (5.60 mL) was added 28 $\mathrm{wt} \% \mathrm{NaOMe}(53.5 \mathrm{mg}, 0.278 \mathrm{mmol})$ in three portions. After stirring for $7 \mathrm{~h}$ at ambient temperature and for $42.5 \mathrm{~h}$ at $40{ }^{\circ} \mathrm{C}$ as the progress of the reaction was monitored by TLC ( $n$ hexane/toluene/EtOAc $=5 / 3 / 1)$, the reaction mixture was neutralized with Muromac $\left(\mathrm{H}^{+}\right)$resin and filtered through cotton. The resin was washed with $\mathrm{MeOH}$. The filtrate was concentrated. The residue was purified by silica gel column chromatography ( $n$-hexane/toluene/EtOAc $=7 / 2 / 1 \rightarrow 5 / 3 / 1$ ) to give compound S13 (336 mg, 97\%) as a colorless syrup; $[\alpha]_{D}-12.5^{\circ}$ (c 1.0, $\mathrm{CHCl}_{3}$ ); ${ }^{1} \mathrm{H} \mathrm{NMR}(500 \mathrm{MHz}$, $\left.\mathrm{CDCl}_{3}\right) \delta 7.56-6.84(\mathrm{~m}, 13 \mathrm{H}, 3 \mathrm{Ar}), 4.82\left(\mathrm{~d}, 1 \mathrm{H}, \mathrm{J}_{\mathrm{gem}}=11.0 \mathrm{~Hz}, \mathrm{ArCH}_{2}\right), 4.69\left(\mathrm{~d}, 1 \mathrm{H}, \mathrm{ArCH}_{2}\right), 4.56$ (d, 1 $\left.\mathrm{H}, J_{\text {gem }}=11.5 \mathrm{~Hz}, \mathrm{ArCH}_{2}\right), 4.53\left(\mathrm{~d}, 1 \mathrm{H}, J_{1,2}=9.5 \mathrm{~Hz}, \mathrm{H}-1\right), 4.45\left(\mathrm{~d}, 1 \mathrm{H}, \mathrm{ArCH}_{2}\right), 3.81(\mathrm{~s}, 3 \mathrm{H}, \mathrm{OMe}), 3.79$ (s, $3 \mathrm{H}, \mathrm{OMe}$ ), 3.77 (dd, $\left.1 \mathrm{H}, J_{5,6 \mathrm{a}}=2.0 \mathrm{~Hz}, J_{\text {gem }}=10.5 \mathrm{~Hz}, \mathrm{H}-6 \mathrm{a}\right), 3.59-3.54(\mathrm{~m}, 2 \mathrm{H}, \mathrm{H}-4, \mathrm{H}-6 \mathrm{~b}), 3.50-$ $3.43(\mathrm{~m}, 2 \mathrm{H}, \mathrm{H}-2, \mathrm{H}-5), 3.36$ (t, $1 \mathrm{H}, J_{2,3}=J_{3,4}=8.3 \mathrm{~Hz}, \mathrm{H}-3$ ), 2.26 (d, $1 \mathrm{H}, J_{2, \mathrm{OH}}=2.0 \mathrm{~Hz}, \mathrm{OH}-2$ ). 0.86 (s, $\left.9 \mathrm{H}, \mathrm{OSi}\left({ }^{t} \mathrm{Bu}\right) \mathrm{Me}_{2}\right), 0.04\left(\mathrm{~s}, 3 \mathrm{H}, \mathrm{OSi}\left({ }^{\mathrm{t} B u}\right) \mathrm{Me}_{2}\right), 0.00\left(\mathrm{~s}, 3 \mathrm{H}, \mathrm{OSi}\left({ }^{t} \mathrm{Bu}\right) \mathrm{Me}_{2}\right) ;{ }^{13} \mathrm{C} \mathrm{NMR}\left(125 \mathrm{MHz}, \mathrm{CDCl}_{3}\right) \delta$ $159.1,159.1,132.4,132.3,130.8,130.6,129.4,129.1,128.9,127.8,113.8,113.7,88.1,86.0,80.8$, 74.6, 73.0, 72.9, 70.6, 69.2, 55.3, 55.2, 25.9, 18.0, -3.7, -4.8; HRMS (ESI) $\mathrm{m} / \mathrm{z}$ : found $[\mathrm{M}+\mathrm{Na}]^{+}$ $649.2624, \mathrm{C}_{34} \mathrm{H}_{46} \mathrm{O}_{7} \mathrm{SSi}$ calcd for $[\mathrm{M}+\mathrm{Na}]^{+} 649.2626$. 


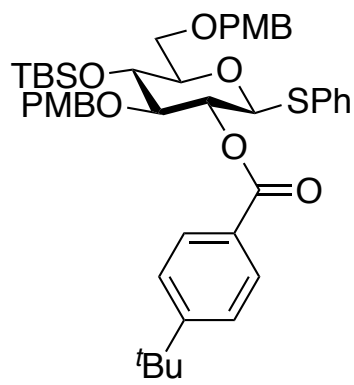

Phenyl

2-O-p-tert-butylbenzoyl-4-O-tert-butyldimethylsilyl-3,6-di-O-pmethoxybenzyl-1-thio- $\beta$-D-glucopyranoside (14b)

To a solution of compound $\mathbf{S 1 3}(322 \mathrm{mg}, 0.514 \mathrm{mmol})$ in pyridine $(5.10 \mathrm{~mL})$ was added p-tertbutylbenzoyl chloride (187 $\mu \mathrm{L}, 1.03 \mathrm{mmol})$ at $0{ }^{\circ} \mathrm{C}$. After stirring for $19 \mathrm{~h}$ at ambient temperature and for $25 \mathrm{~h}$ at $40{ }^{\circ} \mathrm{C}$ as the progress of the reaction was monitored by TLC ( $n$-hexane/EtOAC $=3 / 1$ ), the reaction was quenched with $\mathrm{MeOH}$. The reaction mixture was co-evaporated with toluene and extracted with $\mathrm{CHCl}_{3}$. The organic layer was washed with $2 \mathrm{M} \mathrm{HCl}, \mathrm{H}_{2} \mathrm{O}$, satd. aq. $\mathrm{NaHCO}_{3}$ and brine, dried over $\mathrm{Na}_{2} \mathrm{SO}_{4}$, and concentrated. The residue was purified by silica gel column chromatography $(n$-hexane/EtOAC $=10 / 1 \rightarrow 8 / 1)$ to give compound $14 \mathrm{~b}(352 \mathrm{mg}, 87 \%)$ as a colorless syrup; $[\alpha]_{D}+58.3^{\circ}$ (c 1.0, $\left.\mathrm{CHCl}_{3}\right) ;{ }^{1} \mathrm{H}$ NMR $\left(500 \mathrm{MHz}, \mathrm{CDCl}_{3}\right) \delta 7.93-6.64(\mathrm{~m}, 17 \mathrm{H}, 4 \mathrm{Ar}), 5.28\left(\mathrm{t}, 1 \mathrm{H}, \mathrm{J}_{1,2}=J_{2,3}=9.3 \mathrm{~Hz}, \mathrm{H}-\right.$ 2), $4.83(\mathrm{~d}, 1 \mathrm{H}, \mathrm{H}-1), 4.58-4.54\left(\mathrm{~m}, 3 \mathrm{H}, 3 \mathrm{ArCH}_{2}\right), 4.46\left(\mathrm{~d}, 1 \mathrm{H}, J_{\mathrm{gem}}=11.5 \mathrm{~Hz}, \operatorname{ArCH}_{2}\right), 3.81-3.79(\mathrm{~m}$, $4 \mathrm{H}, \mathrm{H}-6 \mathrm{a}, \mathrm{OMe}), 3.70-3.64$ (m, $5 \mathrm{H}, \mathrm{H}-3, \mathrm{H}-4, \mathrm{OMe}), 3.61-3.57$ (m, $2 \mathrm{H}, \mathrm{H}-5, \mathrm{H}-6 \mathrm{~b}), 1.33$ (s, $\left.9 \mathrm{H},{ }^{t} \mathrm{Bu}\right)$, $0.86\left(\mathrm{~s}, 9 \mathrm{H}, \mathrm{OSi}\left({ }^{\mathrm{t} B u}\right) \mathrm{Me}_{2}\right), 0.00\left(\mathrm{~s}, 3 \mathrm{H}, \mathrm{OSi}\left({ }^{\mathrm{t}} \mathrm{Bu}\right) \mathrm{Me}_{2}\right),-0.02\left(\mathrm{~s}, 3 \mathrm{H}, \mathrm{OSi}\left({ }^{t} \mathrm{Bu}\right) \mathrm{Me}_{2}\right) ;{ }^{13} \mathrm{C} \mathrm{NMR}(125 \mathrm{MHz}$, $\left.\mathrm{CDCl}_{3}\right) \delta 165.2,159.1,158.8,156.8,133.6,131.9,130.5,130.1,129.7,129.3,129.2,128.7,127.4$, 127.1, 125.3, 113.7, 113.4, 86.2, 84.5, 80.8, 74.8, 73.1, 72.7, 71.1, 69.3, 55.3, 55.1, 35.1, 31.1, 25.9, 18.0, -3.7, -4.7; HRMS (ESI) $\mathrm{m} / \mathrm{z}$ : found [M+Na] $]^{+} 809.3511, \mathrm{C}_{45} \mathrm{H}_{58} \mathrm{O}_{8} \mathrm{SSi}$ calcd for $[\mathrm{M}+\mathrm{Na}]^{+} 809.3514$.

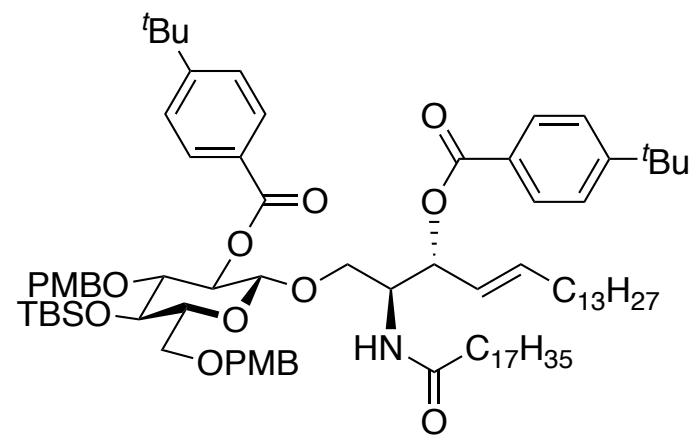

(2-O-p-tert-Butylbenzoyl-4-O-tert-butyldimethylsilyl-3,6-di-O-p-methoxybenzyl- $\beta$-Dglucopyranosyl)-(1 $\rightarrow 1)-(2 S, 3 R, 4 E)-3-O-p$-tert-butylbenzoyl-2-octadecanamido-4octadecene-1,3-diol (16b) 
To a solution of glycosyl donor $14 \mathbf{b}(860 \mathrm{mg}, 1.09 \mathrm{mmol})$ and acceptor $15 \mathrm{~b}(792 \mathrm{mg}, 1.09 \mathrm{mmol})$ in $\mathrm{CH}_{2} \mathrm{Cl}_{2}(15.0 \mathrm{~mL})$ were added $4 \AA$ molecular sieves $(3.27 \mathrm{~g})$ and 2,4,6-tri-tert-butylpyrimidine (1.08 g, $4.36 \mathrm{mmol}$ ). After stirring for $1 \mathrm{~h}$ at ambient temperature, the mixture was cooled to $0{ }^{\circ} \mathrm{C}$. To the mixture was added dropwise dimethyl(methylthio)sulfonium trifluoromethanesulfonate (DMTST), which was pre-prepared in situ by reacting MeSSMe $(581 \mu \mathrm{L}, 6.54 \mathrm{mmol}$ ) and MeOTf (358 $\mu \mathrm{L}, 3.27$ mmol) for $5 \mathrm{~min}$ in $\mathrm{CH}_{2} \mathrm{Cl}_{2}\left(5.00 \mathrm{~mL}\right.$ ), followed by washing the flask containing of DMTST with $\mathrm{CH}_{2} \mathrm{Cl}_{2}$ $(1.80 \mathrm{~mL})$ and transfer to the reaction mixture. After stirring for $2 \mathrm{~h}$ at ambient temperature as the progress of the reaction was monitored by TLC ( $n$-hexane/toluene/EtOAC $=4 / 2 / 1$ ), the reaction mixture was neutralized with satd. aq. $\mathrm{NaHCO}_{3}$ and filtered through a pad of Celite. The pad was washed with $\mathrm{CHCl}_{3}$. The combined filtrate and washings were diluted with $\mathrm{CHCl}_{3}$ and washed with satd. aq. $\mathrm{NaHCO}_{3}, \mathrm{H}_{2} \mathrm{O}$ and brine. The organic layer was dried over $\mathrm{Na}_{2} \mathrm{SO}_{4}$ and concentrated. The residue was purified by silica gel column chromatography $\left(1^{\text {st: }} n\right.$-hexane/toluene/EtOAc $=5 / 3 / 1,2^{\text {nd }}$ : toluene/EtOAC $=30 / 1 \rightarrow 20 / 1)$ to give compound $16 \mathrm{~b}(1.15 \mathrm{~g}, 75 \%)$ as a colorless syrup; $[\alpha]_{D}+26.3^{\circ}$ (c 1.0, $\left.\mathrm{CHCl}_{3}\right) ;{ }^{1} \mathrm{H}$ NMR (500 MHz, $\left.\mathrm{CDCl}_{3}\right)$ 8 7.96-6.65 (m, $16 \mathrm{H}, 4 \mathrm{Ar}$ ), 5.84-5.79 (m, $2 \mathrm{H}, \mathrm{H}-5^{\mathrm{Cer}}$, NH$2^{\text {Cer }), ~} 5.52\left(\mathrm{t}, 1 \mathrm{H}, J_{2,3}=J_{3,4}=7.3 \mathrm{~Hz}, \mathrm{H}-3^{\mathrm{Cer}}\right.$ ), $5.44\left(\mathrm{dd}, 1 \mathrm{H}, J_{4,5}=15.0 \mathrm{~Hz}, \mathrm{H}-4^{\mathrm{Cer}}\right), 5.22\left(\mathrm{t}, 1 \mathrm{H}, J_{1,2}=J_{2,3}\right.$ $\left.=8.3 \mathrm{~Hz}, \mathrm{H}-2^{G / c}\right), 4.59\left(\mathrm{~d}, 1 \mathrm{H}, J_{\text {gem }}=11.0 \mathrm{~Hz}, \operatorname{ArCH}_{2}\right), 4.56\left(\mathrm{~d}, 1 \mathrm{H}, \mathrm{ArCH}_{2}\right), 4.50\left(\mathrm{~d}, 1 \mathrm{H}, \mathrm{H}-1^{G / c}\right), 4.40-$ $4.36\left(\mathrm{~m}, 2 \mathrm{H}, \mathrm{H}-2^{\mathrm{Cer}}, \mathrm{ArCH}_{2}\right), 4.24\left(\mathrm{~d}, 1 \mathrm{H}, \mathrm{J}_{\mathrm{gem}}=12.0 \mathrm{~Hz}, \mathrm{ArCH}_{2}\right), 4.12\left(\mathrm{dd}, 1 \mathrm{H}, \mathrm{J}_{1 \mathrm{a}, 2}=3.3 \mathrm{~Hz}, J_{\text {gem }}=9.8\right.$ $\mathrm{Hz}, \mathrm{H}-1 \mathrm{a}^{\mathrm{Cer}}$ ), 3.78 (s, $\left.3 \mathrm{H}, \mathrm{OMe}\right), 3.70(\mathrm{~s}, 3 \mathrm{H}, \mathrm{OMe}), 3.69-3.62\left(\mathrm{~m}, 3 \mathrm{H}, \mathrm{H}-3^{G / c}, \mathrm{H}-4^{G / c}, \mathrm{H}-6 a^{G / c}\right), 3.58$ (dd, $\left.1 \mathrm{H}, J_{1 b, 2}=3.8 \mathrm{~Hz}, \mathrm{H}-1 \mathrm{~b}^{\mathrm{Cer}}\right), 3.49-3.43\left(\mathrm{~m}, 2 \mathrm{H}, \mathrm{H}-5^{G / c}, \mathrm{H}-6 \mathrm{~b}^{G l c}\right), 1.98-1.94\left(\mathrm{~m}, 2 \mathrm{H}, \mathrm{H}-6 \mathrm{a}^{\mathrm{Cer}}, \mathrm{H}-6 \mathrm{~b}^{\mathrm{Cer}}\right)$, $1.72\left(\mathrm{t}, 2 \mathrm{H}, \mathrm{NHCOCH}_{2}{ }^{\mathrm{Cer}}\right), 1.39-1.10\left(\mathrm{~m}, 70 \mathrm{H}, 26 \mathrm{CH}_{2}{ }^{\mathrm{Cer}}, 2^{\mathrm{t}} \mathrm{Bu}\right), 0.89-0.86\left(\mathrm{~m}, 6 \mathrm{H}, 2 \mathrm{Me}^{\mathrm{Cer}}\right), 0.82(\mathrm{~s}$, $\left.9 \mathrm{H}, \mathrm{OSi}\left({ }^{\mathrm{t} B u}\right) \mathrm{Me}_{2}\right),-0.02\left(\mathrm{~s}, 3 \mathrm{H}, \mathrm{OSi}\left({ }^{\mathrm{t}} \mathrm{Bu}\right) \mathrm{Me}_{2}\right),-0.03\left(\mathrm{~s}, 3 \mathrm{H}, \mathrm{OSi}\left({ }^{\mathrm{t} B u}\right) \mathrm{Me}_{2}\right) ;{ }^{13} \mathrm{CNMR}\left(125 \mathrm{MHz}, \mathrm{CDCl}_{3}\right)$ $\delta 172.7,165.4,165.2,159.1,158.8,157.0,156.4,136.9,130.3,130.2,129.6,129.6,129.2,129.1$, $127.7,126.9,125.4,125.3,125.0,113.7,113.4,100.8,82.8,74.5,74.4,74.3,73.1,71.1,69.0,66.9$, 55.2, 55.1, 50.5, 36.4, 35.1, 35.0, 32.3, 31.9, 31.1, 31.1, 29.7, 29.7, 29.7, 29.7, 29.6, 29.6, 29.5, 29.4, 29.3, 29.3, 29.0, 25.9, 25.6, 22.7, 18.0, 14.1, -3.8, -4.8; HRMS (ESI) $\mathrm{m} / \mathrm{z}$ : found [M+Na] ${ }^{+}$1424.9646, $\mathrm{C}_{86} \mathrm{H}_{135} \mathrm{NO}_{12} \mathrm{~S}$ calcd for $[\mathrm{M}+\mathrm{Na}]^{+} 1424.9646$. 


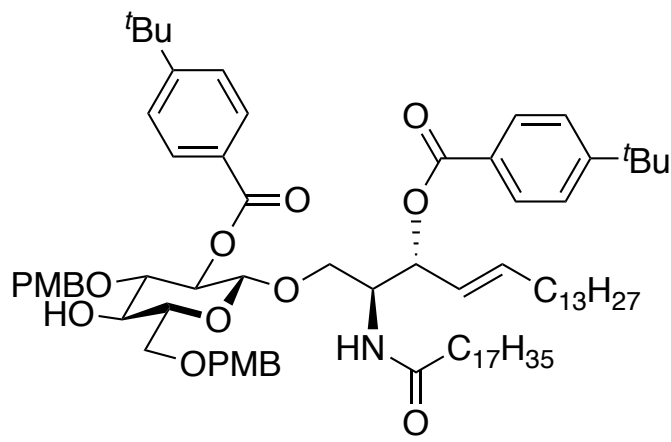

(2-O-p-tert-Butylbenzoyl-3,6-di-O-p-methoxybenzyl- $\beta$-D-glucopyranosyl)-(1 $\rightarrow 1)$ -

(2S,3R,4E)-3-O-p-tert-butylbenzoyl-2-octadecanamido-4-octadecene-1,3-diol (17b)

To a solution of compound 16b (432 mg, $0.308 \mathrm{mmol})$ in THF $(6.20 \mathrm{~mL})$ were added AcOH $(21.1 \mu \mathrm{L}$, $0.369 \mathrm{mmol})$ and $1 \mathrm{M} \mathrm{TBAF}$ in THF $(0.92 \mathrm{~mL}, 0.924 \mathrm{mmol})$. After stirring for $6 \mathrm{~h}$ at ambient temperature as the progress of the reaction was monitored by TLC $(n$-hexane/EtOAC $=2 / 1)$, the reaction mixture was extracted with EtOAc. The organic layer was washed with $\mathrm{H}_{2} \mathrm{O}$ and brine, dried over $\mathrm{Na}_{2} \mathrm{SO}_{4}$, and concentrated. The residue was purified by silica gel column chromatography ( $n$-hexane/EtOAc $=3 / 1$ $\rightarrow 1 / 1$ ) to give compound $17 \mathrm{~b}$ (369 $\mathrm{mg}, 93 \%$ ) as a white amorphous solid; $[\alpha]_{D}+9.8^{\circ}$ (c $1.0, \mathrm{CHCl}_{3}$ ); ${ }^{1} \mathrm{H} \mathrm{NMR}\left(500 \mathrm{MHz}, \mathrm{CDCl}_{3}\right) \delta 7.94-6.70\left(\mathrm{~m}, 16 \mathrm{H}, 4 \mathrm{Ar}\right.$ ), 5.81 (near quin, $1 \mathrm{H}, J_{5,6 \mathrm{a}}=J_{5,6 \mathrm{~b}}=6.5 \mathrm{~Hz}, J_{4,5}=$ $15.5 \mathrm{~Hz}, \mathrm{H}-5^{\mathrm{Cer}}$ ), 5.73 (d, $1 \mathrm{H}, J_{2, \mathrm{NH}}=9.5 \mathrm{~Hz}, \mathrm{NH}-2^{\mathrm{Cer}}$ ), 5.49 (t, $1 \mathrm{H}, J_{2,3}=J_{3,4}=7.3 \mathrm{~Hz}, \mathrm{H}-3^{\mathrm{Cer}}$ ), 5.42 (dd, 1 $\mathrm{H}, \mathrm{H}-4^{\mathrm{Cer}}$ ), 5.17 (dd, $1 \mathrm{H}, J_{1,2}=7.8 \mathrm{~Hz}, J_{2,3}=9.3 \mathrm{~Hz}, \mathrm{H}-2^{G / c}$ ), $4.67\left(\mathrm{~d}, 1 \mathrm{H}, J_{\text {gem }}=11.5 \mathrm{~Hz}, \mathrm{ArCH}_{2}\right.$ ), 4.60 (d, $\left.1 \mathrm{H}, \mathrm{ArCH}_{2}\right), 4.45\left(\mathrm{~d}, 1 \mathrm{H}, \mathrm{H}-1^{G / c}\right), 4.40-4.35\left(\mathrm{~m}, 2 \mathrm{H}, \mathrm{H}-2^{\mathrm{Cer}}, \mathrm{ArCH}_{2}\right), 4.33\left(\mathrm{~d}, 1 \mathrm{H}, J_{\text {gem }}=11.5 \mathrm{~Hz}, \mathrm{ArCH}_{2}\right)$, $4.06\left(\mathrm{dd}, 1 \mathrm{H}, \mathrm{J}_{1 \mathrm{a}, 2}=3.0 \mathrm{~Hz}, \mathrm{~J}_{\mathrm{gem}}=10.0 \mathrm{~Hz}, \mathrm{H}-1 \mathrm{a}^{\mathrm{Cer}}\right), 3.79(\mathrm{~s}, 3 \mathrm{H}, \mathrm{OMe}), 3.75-3.71\left(\mathrm{~m}, 4 \mathrm{H}, \mathrm{H}-4^{G l c}, \mathrm{OMe}\right)$, 3.65-3.61 (m, $\left.2 \mathrm{H}, \mathrm{H}-3^{G / c}, \mathrm{H}-6 \mathrm{a}^{G / c}\right), 3.60-3.54\left(\mathrm{~m}, 2 \mathrm{H}, \mathrm{H}-1 \mathrm{~b}^{\mathrm{Cer}}, \mathrm{H}-6 \mathrm{~b}^{G / c}\right), 3.46$ (near dt, $1 \mathrm{H}, J_{5,6 \mathrm{a}}=J_{5,6 \mathrm{~b}}$ $\left.=4.8 \mathrm{~Hz}, J_{4,5}=9.5 \mathrm{~Hz}, \mathrm{H}-5^{G / c}\right), 2.78\left(\mathrm{~d}, 1 \mathrm{H}, \mathrm{J}_{4, \mathrm{OH}}=2.0 \mathrm{~Hz}, \mathrm{OH}-4^{G / c}\right), 1.98-1.94\left(\mathrm{~m}, 2 \mathrm{H}, \mathrm{H}-6 \mathrm{a}^{\mathrm{Cer}}, \mathrm{H}-6 \mathrm{~b}^{\mathrm{Cer}}\right)$, 1.76-1.73 (m, $\left.2 \mathrm{H}, \mathrm{NHCOCH}_{2}{ }^{\mathrm{Cer}}\right), 1.39-1.09\left(\mathrm{~m}, 70 \mathrm{H}, 26 \mathrm{CH}_{2}{ }^{\mathrm{Cer}},{ }^{\mathrm{t}} \mathrm{Bu}\right), 0.89-0.86\left(\mathrm{~m}, 6 \mathrm{H}, 2 \mathrm{Me}^{\mathrm{Cer}}\right) ;{ }^{13} \mathrm{C}$ NMR $\left(125 \mathrm{MHz}, \mathrm{CDCl}_{3}\right) \delta$ 172.7, 165.4, 165.1, 159.3, 159.2, 157.1, 156.5, 137.1, 130.2, 129.7, 129.6, 129.6, 129.4, 127.7, 126.9, 125.5, 125.3, 125.0, 113.9, 113.8, 101.0, 81.4, 74.2, 74.1, 73.9, 73.6, 73.5, 72.5, 70.3, 67.2, 55.3, 55.2, 50.4, 36.5, 35.1, 35.1, 32.3, 31.9, 31.1, 31.1, 29.7, 29.7, 29.7, 29.6, 29.5, 29.5, 29.4, 29.3, 29.3, 29.0, 25.6, 22.7, 14.1; HRMS (ESI) $\mathrm{m} / \mathrm{z}$ : found $[\mathrm{M}+\mathrm{Na}]^{+} 1310.8784, \mathrm{C}_{80} \mathrm{H}_{121} \mathrm{NO}_{12}$ calcd for $[\mathrm{M}+\mathrm{Na}]^{+} 1310.8781$. 


\subsection{Gal donor}

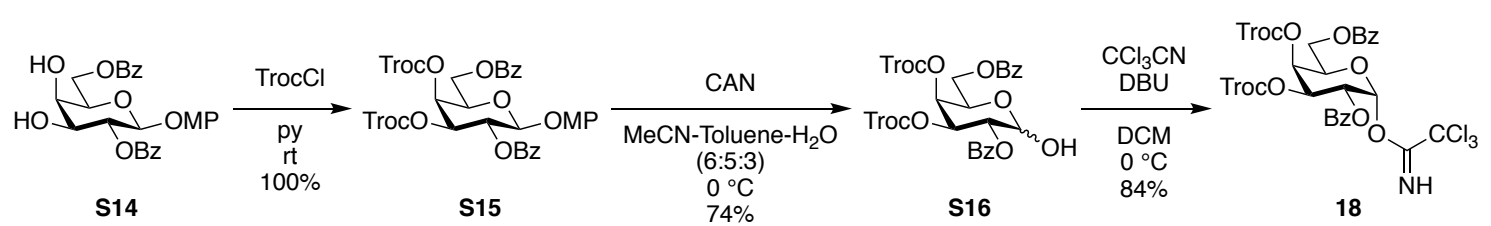

Supporting Scheme 9. Synthesis of Gal donor 18

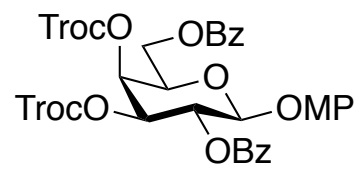

p-Methoxyphenyl 2,6-di-O-benzoyl-3,4-di-O-(2,2,2-trichloroethoxycarbonyl)- $\beta$-Dgalactopyranoside (S15)

To a solution of compound $\mathbf{S 1 4}{ }^{\mathrm{S} 06}(60.0 \mathrm{mg}, 0.121 \mathrm{mmol})$ in pyridine $(1.20 \mathrm{~mL})$ was added $2,2,2-$ trichloroethyl chloroformate $(50.1 \mu \mathrm{L}, 0.364 \mathrm{mmol})$ at $0{ }^{\circ} \mathrm{C}$. After stirring for $30 \mathrm{~min}$ at ambient temperature as the progress of the reaction was monitored by TLC $(n$-hexane/EtOAc $=2 / 1)$, the reaction was quenched with $\mathrm{MeOH}$. The reaction mixture was co-evaporated with toluene and the resulting residue was extracted with $\mathrm{CHCl}_{3}$. The organic layer was washed with $2 \mathrm{M} \mathrm{HCl}, \mathrm{H}_{2} \mathrm{O}$, satd. aq. $\mathrm{NaHCO}_{3}$ and brine, dried over $\mathrm{Na}_{2} \mathrm{SO}_{4}$, and concentrated. The residue was purified by silica gel column chromatography $(n$-hexane/EtOAc $=3 / 1)$ to give compound S15 $(102 \mathrm{mg}, 100 \%)$ as a white foam; $[\alpha]_{D}$ $+22.7^{\circ}$ (c 1.0, $\mathrm{CHCl}_{3}$ ); ${ }^{1} \mathrm{H}$ NMR (500 MHz, $\mathrm{CDCl}_{3}$ ) $\delta 8.04-6.67$ (m, $14 \mathrm{H}, 3 \mathrm{Ar}$ ), 5.87 (dd, $1 \mathrm{H}, \mathrm{J}_{1,2}=8.0$ $\mathrm{Hz}, J_{2,3}=10.1 \mathrm{~Hz}, \mathrm{H}-2$ ), 5.59 (d, $1 \mathrm{H}, J_{3,4}=3.4 \mathrm{~Hz}, \mathrm{H}-4$ ), 5.30 (dd, $1 \mathrm{H}, \mathrm{H}-3$ ), 5.11 (d, $1 \mathrm{H}, \mathrm{H}-1$ ), 4.86 (d, $\left.1 \mathrm{H}, J_{\text {gem }}=11.8 \mathrm{~Hz}, \mathrm{C}(\mathrm{O}) \mathrm{OCH}_{2} \mathrm{CCl}_{3}\right), 4.81\left(\mathrm{~d}, 1 \mathrm{H}, \mathrm{C}(\mathrm{O}) \mathrm{OCH}_{2} \mathrm{CCl}_{3}\right), 4.74\left(\mathrm{~d}, 1 \mathrm{H}, J_{\text {gem }}=11.8 \mathrm{~Hz}\right.$, $\left.\mathrm{C}(\mathrm{O}) \mathrm{OCH}_{2} \mathrm{CCl}_{3}\right), 4.68\left(\mathrm{dd}, 1 \mathrm{H}, J_{5,6 \mathrm{a}}=7.0 \mathrm{~Hz}, \mathrm{~J}_{\mathrm{gem}}=11.4 \mathrm{~Hz}, \mathrm{H}-6 \mathrm{a}\right), 4.63\left(\mathrm{~d}, 1 \mathrm{H}, \mathrm{C}(\mathrm{O}) \mathrm{OCH}_{2} \mathrm{CCl}_{3}\right), 4.52$ (dd, $1 \mathrm{H}, J_{5,6 b}=6.3 \mathrm{~Hz}, \mathrm{H}-6 \mathrm{~b}$ ), 4.27 (near t, $1 \mathrm{H}, \mathrm{H}-5$ ), 3.72 (s, $3 \mathrm{H}, \mathrm{OMe}$ ); ${ }^{13} \mathrm{C} \mathrm{NMR}\left(125 \mathrm{MHz}, \mathrm{CDCl}_{3}\right.$ ) $\delta$ 165.9, 164.8, 155.9, 154.1, 153.3, 150.9, 133.5, 129.9, 129.8, 129.2, 129.1, 128.6, 128.5, 119.1, 114.5, $101.2,94.2,93.8,77.2,77.1,75.5,71.9,70.7,69.2,61.5,55.6$; HRMS (ESI) $\mathrm{m} / \mathrm{z}$ : found $[\mathrm{M}+\mathrm{Na}]^{+}$ $864.9553, \mathrm{C}_{33} \mathrm{H}_{28} \mathrm{Cl}_{6} \mathrm{O}_{13}$ calcd for $[\mathrm{M}+\mathrm{Na}]^{+} 864.9553$.

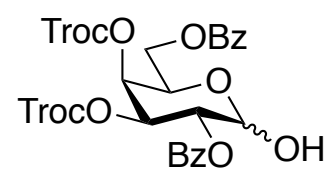

2,6-Di-O-benzoyl-3,4-di-O-(2,2,2-trichloroethoxycarbonyl)-D-galactopyranose (S16)

To a solution of compound $\mathbf{S 1 5}\left(91.8 \mathrm{mg}, 0.109 \mathrm{mmol}\right.$ ) in $\mathrm{MeCN} /$ toluene/ $\mathrm{H}_{2} \mathrm{O}=6 / 5 / 3(2.20 \mathrm{~mL}$ ) was 
added CAN (598 mg, $1.09 \mathrm{mmol}$ ). After vigorously stirring for $1 \mathrm{~h}$ at $0^{\circ} \mathrm{C}$ as the progress of the reaction was monitored by TLC ( $n$-hexane/EtOAc $=2 / 1)$, the reaction mixture was extracted with EtOAc. The organic layer was washed with $\mathrm{H}_{2} \mathrm{O}$, satd. aq. $\mathrm{NaHCO}_{3}$ and brine, dried over $\mathrm{Na}_{2} \mathrm{SO}_{4}$, and concentrated. The residue was purified by silica gel column chromatography ( $n$-hexane/EtOAc $=3 / 1$ ) and gel filteration ( $\mathrm{LH}-20$, Eluent: $\left.\mathrm{CHCl}_{3} / \mathrm{MeOH}=1 / 1\right)$ to give compound $\mathbf{S 1 6}(59.9 \mathrm{mg}, 74 \%, \alpha: \beta=1: 0.9)$ as a white foam; ${ }^{1} \mathrm{H} N M R\left(500 \mathrm{MHz}, \mathrm{CDCl}_{3}\right) \delta 8.04-7.42(\mathrm{~m}, 20 \mathrm{H}, 2 \mathrm{Ph} \alpha \beta), 5.80\left(\mathrm{t}, 1 \mathrm{H}, J_{1,2}=J_{1, \mathrm{OH}}=3.6 \mathrm{~Hz}\right.$, $\mathrm{H}-1 \alpha), 5.67-5.64(\mathrm{~m}, 2 \mathrm{H}, \mathrm{H}-3 \alpha, \mathrm{H}-4 \alpha), 5.57$ (near $d, 1 \mathrm{H}, \mathrm{H}-4 \beta), 5.48-5.44(\mathrm{~m}, 2 \mathrm{H}, \mathrm{H}-2 \alpha, \mathrm{H}-2 \beta), 5.32$ (dd, $1 \mathrm{H}, J_{2,3}=3.5 \mathrm{~Hz}, J_{3,4}=10.5 \mathrm{~Hz}, \mathrm{H}-3 \beta$ ), 4.90-4.76 (m, $7 \mathrm{H}, \mathrm{H}-1 \beta, 3 \mathrm{C}(\mathrm{O}) \mathrm{OCH}_{2} \mathrm{CCCl}_{3} \alpha \beta$ ), 4.72 (near t, $1 \mathrm{H}, \mathrm{H}-5 \alpha), 4.66\left(\mathrm{dd}, 1 \mathrm{H}, J_{5,6 \mathrm{a}}=6.5 \mathrm{~Hz}, J_{\mathrm{gem}}=11.0 \mathrm{~Hz}, \mathrm{H}-6 \mathrm{a} \beta\right), 4.66-4.61\left(\mathrm{~m}, 2 \mathrm{H}, \mathrm{C}(\mathrm{O}) \mathrm{CH}_{2} \mathrm{CCl}_{3} \alpha \beta\right)$, $4.59\left(\mathrm{dd}, 1 \mathrm{H}, J_{5,6 a}=6.3 \mathrm{~Hz}, J_{\mathrm{gem}}=11.3 \mathrm{~Hz}, \mathrm{H}-6 \mathrm{aa}\right), 4.46\left(\mathrm{dd}, 1 \mathrm{H}, J_{5,6 \mathrm{~b}}=7.3 \mathrm{~Hz}, \mathrm{H}-6 \mathrm{~b} \beta\right), 4.41(\mathrm{dd}, 1 \mathrm{H}$, $J_{5,6 b}=7.3 \mathrm{~Hz}, \mathrm{H}-6 \mathrm{~b} \alpha$ ), 4.22 (near t, $1 \mathrm{H}, \mathrm{H}-5 \beta$ ), 3.99 (d, $1 \mathrm{H}, J_{1, \mathrm{OH}}=9.5 \mathrm{~Hz}, \mathrm{OH}-1 \beta$ ), 3.08 (near d, $1 \mathrm{H}$, $\mathrm{OH}-1 \alpha$ ); HRMS (ESI) $m / z$ : found [M+Na] $]^{+} 758.9137, \mathrm{C}_{26} \mathrm{H}_{22} \mathrm{Cl}_{6} \mathrm{O}_{12}$ calcd for $[\mathrm{M}+\mathrm{Na}]^{+} 758.9135$.

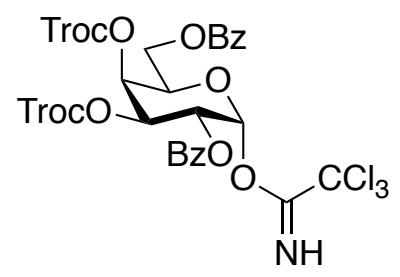

\section{2,6-Di-O-benzoyl-3,4-di-O-(2,2,2-trichloroethoxycarbonyl)- $\alpha$-D-galactopyranosyl trichloroacetimidate (18)}

To a solution of compound $\mathbf{S} 16(50.2 \mathrm{mg}, 67.9 \mu \mathrm{mol})$ in $\mathrm{CH}_{2} \mathrm{Cl}_{2}(1.40 \mathrm{~mL})$ were added $\mathrm{CCl}_{3} \mathrm{CN}(136 \mu \mathrm{L}$, $1.36 \mathrm{mmol})$ and $\mathrm{DBU}(2.0 \mu \mathrm{L}, 13.6 \mu \mathrm{mol})$. After stirring for $1 \mathrm{~h}$ at $0{ }^{\circ} \mathrm{C}$ as the progress of the reaction was monitored by TLC ( $n$-hexane/EtOAC $=2 / 1)$, the reaction mixture was evaporated. The residue was purified by silica gel column chromatography ( $n$-hexane/EtOAc $=5 / 1$ ) to give compound 18 (50.6 $\mathrm{mg}, 84 \%$ ) as a white foam; $[\alpha]_{\mathrm{D}}+63.7^{\circ}$ (c $\left.1.0, \mathrm{CHCl}_{3}\right) ;{ }^{1} \mathrm{H} \mathrm{NMR}\left(500 \mathrm{MHz}, \mathrm{CDCl}_{3}\right) \delta 8.62(\mathrm{~s}, 1 \mathrm{H}$, $\left.\mathrm{C}(=\mathrm{NH}) \mathrm{CCl}_{3}\right), 8.01-7.39(\mathrm{~m}, 10 \mathrm{H}, 2 \mathrm{Ph}), 6.85\left(\mathrm{~d}, 1 \mathrm{H}, \mathrm{J}_{1,2}=3.8 \mathrm{~Hz}, \mathrm{H}-1\right), 5.76-5.73(\mathrm{~m}, 2 \mathrm{H}, \mathrm{H}-2, \mathrm{H}-4)$, $5.67\left(\mathrm{dd}, 1 \mathrm{H}, J_{3,4}=3.3 \mathrm{~Hz}, J_{2,3}=10.8 \mathrm{~Hz}, \mathrm{H}-3\right), 4.85\left(\mathrm{~d}, 1 \mathrm{H}, J_{\mathrm{gem}}=12.0 \mathrm{~Hz}, \mathrm{C}(\mathrm{O}) \mathrm{OCH}_{2} \mathrm{CCl}_{3}\right), 4.81(\mathrm{~d}, 1$ $\left.\mathrm{H}, J_{\text {gem }}=12.0 \mathrm{~Hz}, \mathrm{C}(\mathrm{O}) \mathrm{OCH}_{2} \mathrm{CCl}_{3}\right), 4.78\left(\mathrm{~d}, 1 \mathrm{H}, \mathrm{C}(\mathrm{O}) \mathrm{OCH}_{2} \mathrm{CCl}_{3}\right), 4.70$ (near t, $\left.1 \mathrm{H}, \mathrm{H}-5\right), 4.67(\mathrm{~d}, 1 \mathrm{H}$, $\left.\mathrm{C}(\mathrm{O}) \mathrm{OCH}_{2} \mathrm{CCl}_{3}\right), 4.60\left(\mathrm{dd}, 1 \mathrm{H}, J_{5,6 \mathrm{a}}=6.5 \mathrm{~Hz}, J_{\mathrm{gem}}=11.3 \mathrm{~Hz}, \mathrm{H}-6 \mathrm{a}\right), 4.46\left(\mathrm{dd}, 1 \mathrm{H}, J_{5,6 \mathrm{~b}}=7.0 \mathrm{~Hz}, \mathrm{H}-6 \mathrm{~b}\right.$ ); ${ }^{13} \mathrm{C}$ NMR $\left(125 \mathrm{MHz}, \mathrm{CDCl}_{3}\right) \delta 165.8,165.2,160.3,154.1,153.2,133.7,133.5,129.9,129.8,129.2$, 128.6, 128.5, 128.5, 94.1, 93.8, 93.3, 90.6, 77.2, 77.1, 72.4, 72.3, 68.8, 67.5, 61.5; HRMS (ESI) $\mathrm{m} / \mathrm{z}$ : found $[\mathrm{M}+\mathrm{Na}]^{+} 901.8234, \mathrm{C}_{28} \mathrm{H}_{22} \mathrm{Cl}_{9} \mathrm{NO}_{12}$ calcd for $[\mathrm{M}+\mathrm{Na}]^{+} 901.8231$. 


\subsection{Construction of LacCer skeletons}

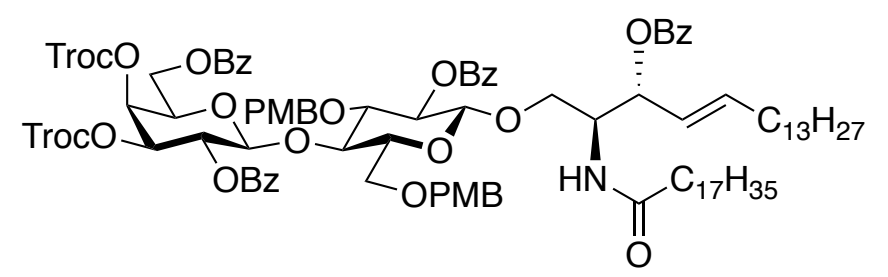

[2,6-Di-O-benzoyl-3,4-di-O-(2,2,2-trichloroethoxycarbonyl)- $\beta$-D-galactopyranosyl]$(1 \rightarrow 4)$-(2-O-benzoyl-3,6-di-O-p-methoxybenzyl- $\beta$-D-glucopyranosyl)-( $1 \rightarrow 1)-(2 S, 3 R, 4 E)$ 3-O-benzoyl-2-octadecanamido-4-octadecene-1,3-diol (19a)

To a solution of donor 18 (20.6 mg, $23.3 \mu \mathrm{mol})$ and acceptor $17 \mathrm{a}(27.4 \mathrm{mg}, 23.3 \mu \mathrm{mol})$ in $\mathrm{CH}_{2} \mathrm{Cl}_{2}(0.93$ $\mathrm{mL}$ ) was added $4 \AA$ Å molecular sieves (AW-300, $144 \mathrm{mg}$ ). After stirring for $1 \mathrm{~h}$ at ambient temperature, TMSOTf $(0.84 \mu \mathrm{L}, 4.66 \mu \mathrm{mol})$ was added to the reaction mixture at $-40^{\circ} \mathrm{C}$. After stirring for $16 \mathrm{~h}$ at $-40{ }^{\circ} \mathrm{C}$ as the progress of the reaction was monitored by TLC $(n$-hexane/EtOAC $=2 / 1)$, the reaction mixture was neutralized with satd. aq. $\mathrm{NaHCO}_{3}$ and filtered through a pad of Celite. The pad was washed with $\mathrm{CHCl}_{3}$. The filtrate was diluted with $\mathrm{CHCl}_{3}$ and washed with brine. The organic layer was dried over $\mathrm{Na}_{2} \mathrm{SO}_{4}$ and concentrated. The residue was purified by silica gel column chromatography $(n$-hexane/EtOAC $=4 / 1 \rightarrow 3 / 1 \rightarrow 1 / 1)$ to give compound $19 \mathrm{a}(12.1 \mathrm{mg}, 27 \%)$ as a colorless syrup; $[\alpha]_{D}$ $+1.1^{\circ}\left(\mathrm{c} 0.9, \mathrm{CHCl}_{3}\right) ;{ }^{1} \mathrm{H} \mathrm{NMR}\left(500 \mathrm{MHz}, \mathrm{CDCl}_{3}\right) \delta 8.00-6.65\left(\mathrm{~m}, 28 \mathrm{H}, 6 \mathrm{Ar}\right.$ ), 5.78 (near quin, $1 \mathrm{H}, J_{5,6 a}$ $\left.=J_{5,6 b}=6.8 \mathrm{~Hz}, J_{4,5}=15.3 \mathrm{~Hz}, \mathrm{H}-5^{c e r}\right), 5.67\left(\mathrm{~d}, 1 \mathrm{H}, J_{2, \mathrm{NH}}=9.0 \mathrm{~Hz}, \mathrm{NH}-2^{\mathrm{Cer}}\right.$ ), 5.59 (dd, $1 \mathrm{H}, J_{1,2}=8.0 \mathrm{~Hz}$, $\left.J_{2,3}=10.5 \mathrm{~Hz}, \mathrm{H}-2^{G a l}\right), 5.48\left(\mathrm{t}, 1 \mathrm{H}, J_{2,3}=J_{3,4}=7.3 \mathrm{~Hz}, \mathrm{H}-3^{\mathrm{Cer}}\right), 5.44-5.39\left(\mathrm{~m}, 2 \mathrm{H}, \mathrm{H}-4^{G a l}, \mathrm{H}-4^{\mathrm{Cer}}\right), 5.18$ (dd, $\left.1 \mathrm{H}, J_{1,2}=8.0 \mathrm{~Hz}, J_{2,3}=9.0 \mathrm{~Hz}, \mathrm{H}-2^{G / c}\right), 5.06\left(\mathrm{dd}, 1 \mathrm{H}, J_{3,4}=3.5 \mathrm{~Hz}, \mathrm{H}-3^{G a l}\right), 4.85-4.83\left(\mathrm{~m}, 2 \mathrm{H}, \mathrm{ArCH}_{2}\right.$, $\left.\mathrm{C}(\mathrm{O}) \mathrm{OCH}_{2} \mathrm{CCl}_{3}\right), 4.79\left(\mathrm{~d}, 1 \mathrm{H}, \mathrm{H}-1^{G a l}\right), 4.76\left(\mathrm{~d}, 1 \mathrm{H}, J_{\text {gem }}=11.5 \mathrm{~Hz}, \mathrm{C}(\mathrm{O}) \mathrm{OCH}_{2} \mathrm{CCl}_{3}\right), 4.75\left(\mathrm{~d}, 1 \mathrm{H}, \mathrm{J}_{\mathrm{gem}}=\right.$ $\left.11.5 \mathrm{~Hz}, \mathrm{C}(\mathrm{O}) \mathrm{OCH}_{2} \mathrm{CCl}_{3}\right), 4.61\left(\mathrm{~d}, 1 \mathrm{H}, \mathrm{J}_{\mathrm{gem}}=10.5 \mathrm{~Hz}, \mathrm{ArCH}_{2}\right), 4.56\left(\mathrm{~d}, 1 \mathrm{H}, \mathrm{C}(\mathrm{O}) \mathrm{OCH}_{2} \mathrm{CCl}_{3}\right), 4.55(\mathrm{~d}, 1 \mathrm{H}$, $\left.J_{\text {gem }}=12.0 \mathrm{~Hz}, \mathrm{ArCH}_{2}\right), 4.38-4.32\left(\mathrm{~m}, 3 \mathrm{H}, \mathrm{H}-1^{G l c}, \mathrm{H}-6 \mathrm{a}^{G a l}, \mathrm{H}-2^{\mathrm{Cer}}\right), 4.28\left(\mathrm{dd}, 1 \mathrm{H}, J_{5,6 \mathrm{~b}}=8.0 \mathrm{~Hz}, J_{\text {gem }}=\right.$ $\left.11.3 \mathrm{~Hz}, \mathrm{H}-6 \mathrm{~b}^{G a l}\right), 4.14-4.08\left(\mathrm{~m}, 2 \mathrm{H}, \mathrm{H}-4^{G l c}, \mathrm{ArCH}_{2}\right), 4.02$ (dd, $1 \mathrm{H}, J_{1 \mathrm{a}, 2}=3.0 \mathrm{~Hz}, J_{\text {gem }}=9.8 \mathrm{~Hz}, \mathrm{H}-1 \mathrm{a}^{\mathrm{Cer}}$ ), 3.82 (near t, $1 \mathrm{H}, \mathrm{H}-5^{\mathrm{Gal}}$ ), 3.77-3.73 (m, $4 \mathrm{H}, \mathrm{H}-3^{\mathrm{Gl}}$, OMe), 3.68 (s, $3 \mathrm{H}, \mathrm{OMe}$ ), 3.55 (dd, $1 \mathrm{H}, \mathrm{J}_{5,6 \mathrm{a}}=3.0$ $\left.\mathrm{Hz}, J_{\text {gem }}=11.0 \mathrm{~Hz}, \mathrm{H}-6 \mathrm{a}^{G / c}\right), 3.48\left(\mathrm{dd}, 1 \mathrm{H}, J_{1 \mathrm{~b}, 2}=4.0 \mathrm{~Hz}, \mathrm{H}-1 \mathrm{~b}^{\mathrm{Cer}}\right), 3.36\left(\mathrm{br} \mathrm{d}, 1 \mathrm{H}, \mathrm{H}-6 \mathrm{~b}^{G / c}\right), 3.23(\mathrm{br} \mathrm{dt}$, $\left.1 \mathrm{H}, J_{4,5}=9.3 \mathrm{~Hz}, \mathrm{H}-5^{G / c}\right), 1.98-1.93\left(\mathrm{~m}, 2 \mathrm{H}, \mathrm{H}-6 \mathrm{a}^{\mathrm{Cer}}, \mathrm{H}-6 \mathrm{~b}^{\mathrm{Cer}}\right), 1.75-1.71\left(\mathrm{~m}, 2 \mathrm{H}, \mathrm{NHCOCH}_{2}{ }^{\mathrm{Cer}}\right), 1.40-$ $1.07\left(\mathrm{~m}, 52 \mathrm{H}, 26 \mathrm{CH}_{2}{ }^{\mathrm{Cer}}\right), 0.89-0.86\left(\mathrm{~m}, 6 \mathrm{H}, 2 \mathrm{Me}^{\mathrm{Cer}}\right) ;{ }^{13} \mathrm{C} \mathrm{NMR}\left(125 \mathrm{MHz}, \mathrm{CDCl}_{3}\right) \delta 172.6,165.7,165.2$, 165.1, 164.5, 159.5, 159.0, 154.1, 153.2, 137.2, 133.6, 133.4, 133.3, 132.8, 130.4, 130.1, 129.8, 129.7, $129.7,129.6$, 129.6, 129.3, 128.9, 128.6, 128.5, 128.5, 128.3, 124.8, 113.9, 113.5, 101.2, 100.2, 94.2, $93.8,79.5,77.0,77.0,76.6,75.6,74.9,74.5,74.5,73.5,73.2,71.9,70.2,69.7,67.4,67.2,60.8,55.2$, 


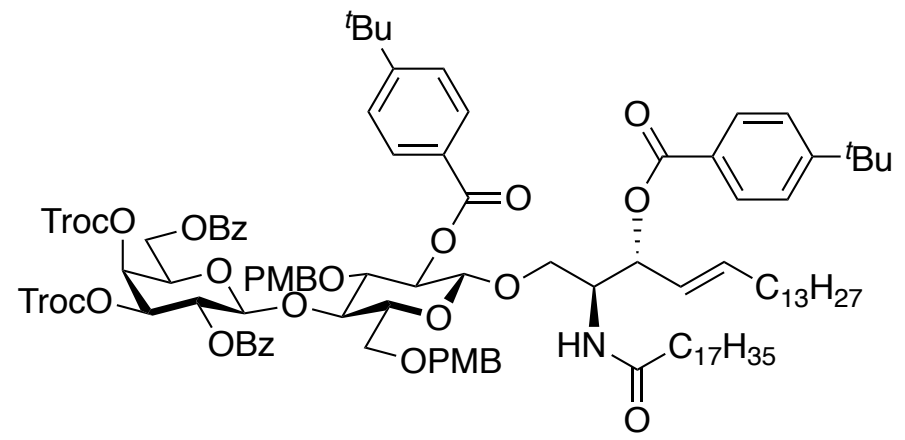

[2,6-Di-O-benzoyl-3,4-di-O-(2,2,2-trichloroethoxycarbonyl)- $\beta$-D-galactopyranosyl]$(1 \rightarrow 4)$-(2-O-p-tert-butylbenzoyl-3,6-di-O-p-methoxybenzyl- $\beta$-D-glucopyranosyl)$(1 \rightarrow 1)-(2 S, 3 R, 4 E)-3-0-p$-tert-butylbenzoyl-2-octadecanamido-4-octadecene-1,3-diol (19b)

To a solution of donor $18(20.6 \mathrm{mg}, 23.3 \mu \mathrm{mol})$ and acceptor $17 \mathrm{~b}(30.0 \mathrm{mg}, 23.3 \mu \mathrm{mol})$ in $\mathrm{CH}_{2} \mathrm{Cl}_{2}(0.93$ $\mathrm{mL}$ ) was added $4 \AA$ Å molecular sieves (AW-300, $152 \mathrm{mg}$ ). After stirring for $1 \mathrm{~h}$ at ambient temperature, TMSOTf $(0.84 \mu \mathrm{L}, 4.66 \mu \mathrm{mol})$ was added to the reaction mixture at $-40^{\circ} \mathrm{C}$. After stirring for $43 \mathrm{~h}$ at $-40{ }^{\circ} \mathrm{C}$ as the progress of the reaction was monitored by TLC ( $n$-hexane/EtOAC $\left.=5 / 2\right)$, the reaction mixture was neutralized with satd. aq. $\mathrm{NaHCO}_{3}$ and filtered through a pad of Celite. The pad was washed with $\mathrm{CHCl}_{3}$. The filtrate was diluted with $\mathrm{CHCl}_{3}$ and washed with brine. The organic layer was dried over $\mathrm{Na}_{2} \mathrm{SO}_{4}$ and concentrated. The residue was purified by silica gel column chromatography ( $n$-hexane/EtOAC $=3 / 1 \rightarrow 5 / 2 \rightarrow 1 / 1)$ to give compound $19 b(35.2 \mathrm{mg}, 75 \%)$ as a colorless syrup; $[\alpha]_{D}$ $+6.8^{\circ}$ (c 1.2, $\mathrm{CHCl}_{3}$ ); $\left.{ }^{1} \mathrm{H} \mathrm{NMR} \mathrm{(500} \mathrm{MHz}, \mathrm{CDCl}_{3}\right) \delta 8.00-6.67\left(\mathrm{~m}, 26 \mathrm{H}, 6 \mathrm{Ar}\right.$ ), 5.78 (near quin, $1 \mathrm{H}, \mathrm{J}_{5,6 \mathrm{a}}$ $=J_{5,6 b}=7.0 \mathrm{~Hz}, J_{4,5}=15.0 \mathrm{~Hz}, \mathrm{H}^{-} 5^{\mathrm{Cer}}$ ), $5.69\left(\mathrm{~d}, 1 \mathrm{H}, J_{2, \mathrm{NH}}=9.0 \mathrm{~Hz}, \mathrm{NH}-2^{\mathrm{Cer}}\right.$ ), $5.58\left(\mathrm{dd}, 1 \mathrm{H}, J_{1,2}=8.0 \mathrm{~Hz}\right.$, $\left.J_{2,3}=10.5 \mathrm{~Hz}, \mathrm{H}-2^{\text {Gal }}\right), 5.47\left(\mathrm{t}, 1 \mathrm{H}, J_{2,3}=J_{3,4}=7.3 \mathrm{~Hz}, \mathrm{H}-3^{\text {Cer }}\right), 5.43-5.39\left(\mathrm{~m}, 2 \mathrm{H}, \mathrm{H}-4^{\text {Gal }}, \mathrm{H}-4^{\text {Cer }}\right), 5.18$ (dd, $\left.1 \mathrm{H}, J_{1,2}=8.0 \mathrm{~Hz}, J_{2,3}=9.0 \mathrm{~Hz}, \mathrm{H}-2^{G / c}\right), 5.05\left(\mathrm{dd}, 1 \mathrm{H}, J_{3,4}=3.5 \mathrm{~Hz}, \mathrm{H}-3^{G a l}\right), 4.84\left(\mathrm{~d}, 1 \mathrm{H}, J_{\text {gem }}=12.0\right.$ $\left.\mathrm{Hz}, \mathrm{C}(\mathrm{O}) \mathrm{OCH}_{2} \mathrm{CCl}_{3}\right), 4.83\left(\mathrm{~d}, 1 \mathrm{H}, \mathrm{J}_{\text {gem }}=11.0 \mathrm{~Hz}, \mathrm{ArCH}_{2}\right), 4.80\left(\mathrm{~d}, 1 \mathrm{H}, \mathrm{H}-1^{\mathrm{Gal}}\right), 4.76\left(\mathrm{~d}, 1 \mathrm{H}, \mathrm{C}(\mathrm{O}) \mathrm{OCH}_{2} \mathrm{CCl}_{3}\right)$, $4.74\left(\mathrm{~d}, 1 \mathrm{H}, J_{\mathrm{gem}}=12.0 \mathrm{~Hz}, \mathrm{C}(\mathrm{O}) \mathrm{OCH}_{2} \mathrm{CCl}_{3}\right), 4.62\left(\mathrm{~d}, 1 \mathrm{H}, \mathrm{ArCH}_{2}\right), 4.56\left(\mathrm{~d}, 1 \mathrm{H}, \mathrm{C}(\mathrm{O}) \mathrm{OCH}_{2} \mathrm{CCl}_{3}\right), 4.54$ (d, $\left.1 \mathrm{H}, J_{\text {gem }}=11.3 \mathrm{~Hz}, \mathrm{ArCH}_{2}\right), 4.38-4.34\left(\mathrm{~m}, 2 \mathrm{H}, \mathrm{H}-1^{\mathrm{Glc}}, \mathrm{H}-2^{\mathrm{Cer}}\right), 4.31\left(\mathrm{dd}, 1 \mathrm{H}, J_{5,6 \mathrm{a}}=6.3 \mathrm{~Hz}, J_{\text {gem }}=11.3\right.$ $\mathrm{Hz}, \mathrm{H}-6 \mathrm{a}^{G a l}$ ), 4.27 (dd, $\left.1 \mathrm{H}, J_{5,6 b}=7.8 \mathrm{~Hz}, \mathrm{H}-6 \mathrm{~b}^{G a l}\right), 4.11\left(\mathrm{t}, 1 \mathrm{H}, J_{3,4}=J_{4,5}=9.0 \mathrm{~Hz}, \mathrm{H}-4^{G / c}\right), 4.09(\mathrm{~d}, 1 \mathrm{H}$, $\operatorname{ArCH}_{2}$ ), 4.01 (dd, $1 \mathrm{H}, J_{1 a, 2}=3.0 \mathrm{~Hz}, J_{\text {gem }}=9.9 \mathrm{~Hz}, \mathrm{H}-1 \mathrm{a}^{\mathrm{Cer}}$ ), 3.80 (near t, $1 \mathrm{H}, \mathrm{H}-5^{\mathrm{Gal}}$ ), 3.77-3.73 (m, 4 $\left.\mathrm{H}, \mathrm{H}-3^{G / c}, \mathrm{OMe}\right), 3.69$ (s, $3 \mathrm{H}, \mathrm{OMe}$ ), $3.55\left(\mathrm{dd}, 1 \mathrm{H}, J_{5,6 a}=3.0 \mathrm{~Hz}, J_{\mathrm{gem}}=11.0 \mathrm{~Hz}, \mathrm{H}-6 \mathrm{a}^{G / c}\right.$ ), $3.47(\mathrm{dd}, 1 \mathrm{H}$, $\left.J_{1 b, 2}=3.8 \mathrm{~Hz}, \mathrm{H}-1 b^{C e r}\right), 3.36\left(\mathrm{br} \mathrm{d}, 1 \mathrm{H}, \mathrm{H}-6 \mathrm{~b}^{G / c}\right), 3.23\left(\mathrm{br} \mathrm{dt}, 1 \mathrm{H}, \mathrm{H}-5^{6 / c}\right), 1.98-1.93\left(\mathrm{~m}, 2 \mathrm{H}, \mathrm{H}-6 \mathrm{a}^{\mathrm{Cer}}, \mathrm{H}-\right.$ 
$\left.6 b^{\mathrm{Cer}}\right), 1.79-1.69\left(\mathrm{~m}, 2 \mathrm{H}, \mathrm{NHCOCH}_{2}{ }^{\mathrm{Cer}}\right), 1.46-1.09\left(\mathrm{~m}, 70 \mathrm{H}, 26 \mathrm{CH}_{2}{ }^{\mathrm{Cer}}, 2{ }^{\mathrm{t}} \mathrm{Bu}\right), 0.89-0.86(\mathrm{~m}, 6 \mathrm{H}, 2$ $\left.\mathrm{Me}^{\mathrm{Cer}}\right) ;{ }^{13} \mathrm{C}$ NMR $\left(125 \mathrm{MHz}, \mathrm{CDCl}_{3}\right) \delta 172.7,165.7,165.3,165.1,164.5,159.5,159.0,157.0,156.4$, 154.1, 153.2, 137.0, 133.6, 133.4, 130.4, 130.2, 129.8, 129.6, 129.6, 129.5, 129.3, 129.0, 128.6, 128.5, $127.6,126.9,125.5,125.3,124.9,113.9,113.5,101.2,100.2$, 94.2, 93.8, 79.6, 77.0, 77.0, 76.6, 75.6, $74.9,74.5,74.2,73.4,73.2,71.9,70.2,69.7,67.4,67.3,60.8,55.2,55.2,50.4,36.5,35.1,35.0,32.3$, 31.9, 31.1, 31.1, 29.7, 29.7, 29.7, 29.6, 29.5, 29.5, 29.4, 29.3, 29.3, 29.0, 25.6, 22.7, 14.1; HRMS (ESI) $\mathrm{m} / \mathrm{z}$ : found $[\mathrm{M}+\mathrm{Na}]^{+} 2028.7914, \mathrm{C}_{106} \mathrm{H}_{141} \mathrm{Cl}_{6} \mathrm{NO}_{23}$ calcd for $[\mathrm{M}+\mathrm{Na}]^{+} 2028.7918$. 
4. ${ }^{1} \mathrm{H}$ and ${ }^{13} \mathrm{C}$ NMR spectral data for new compounds

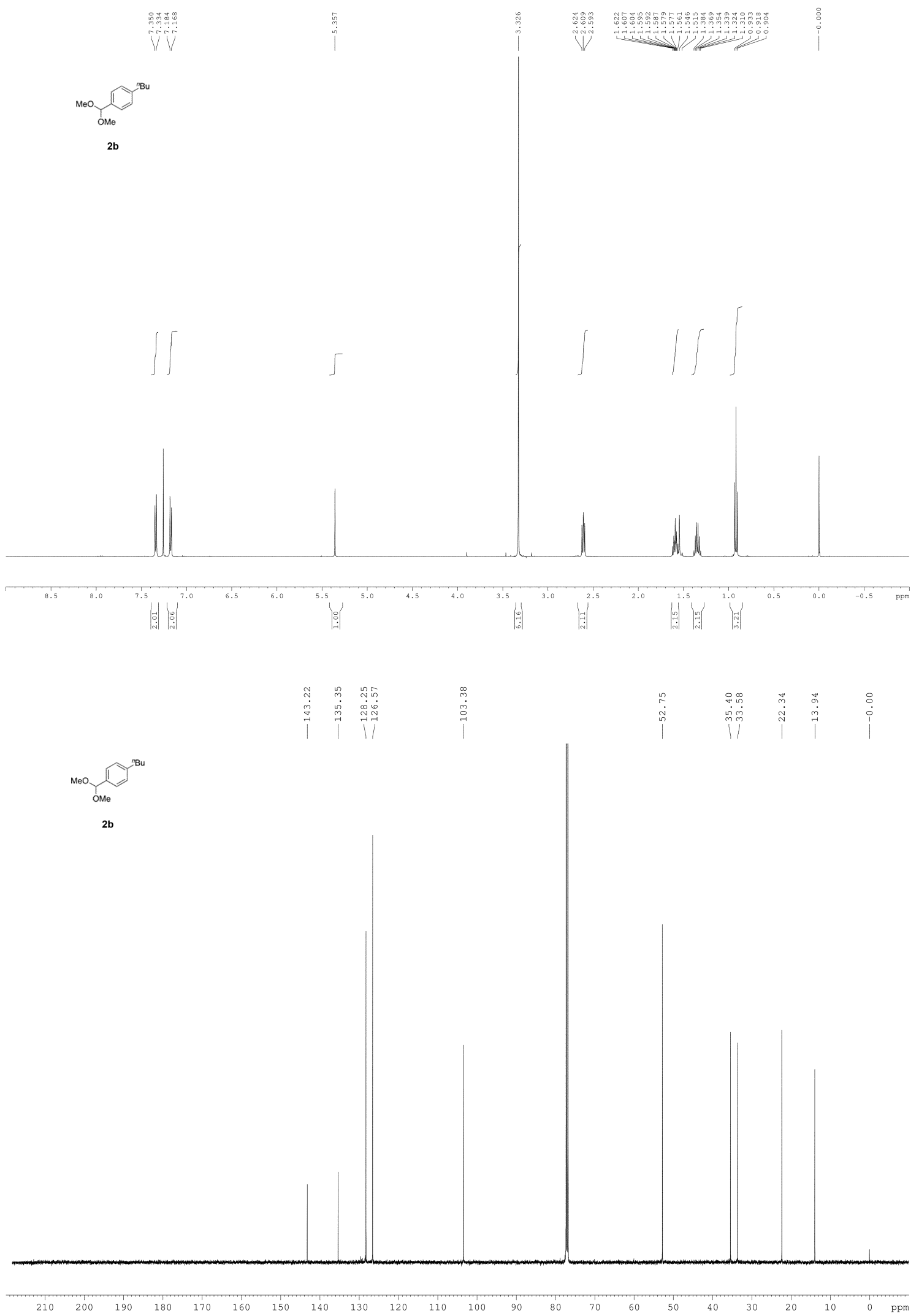




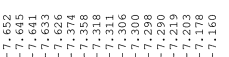

inivinitivin

${ }^{n \mathrm{Bu}}$

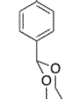

Troco $\underbrace{\text { Oon }}_{\text {NHTroc }}$

$4 \mathrm{~b}$
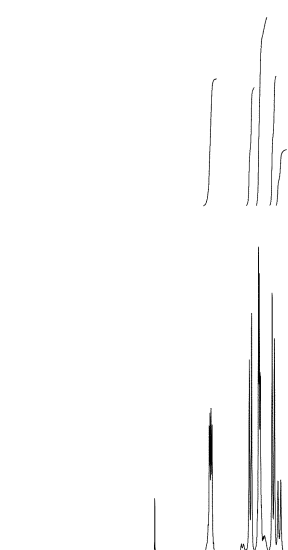

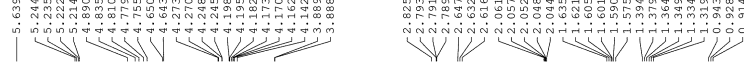

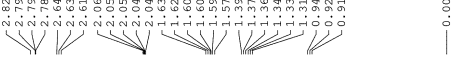

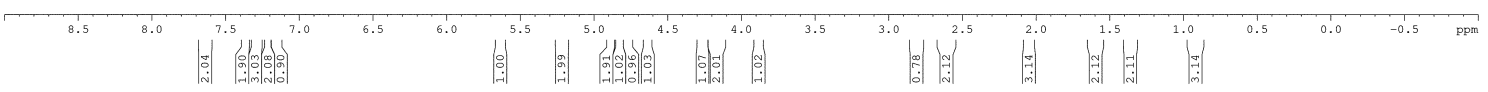

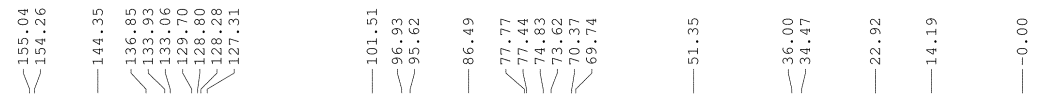

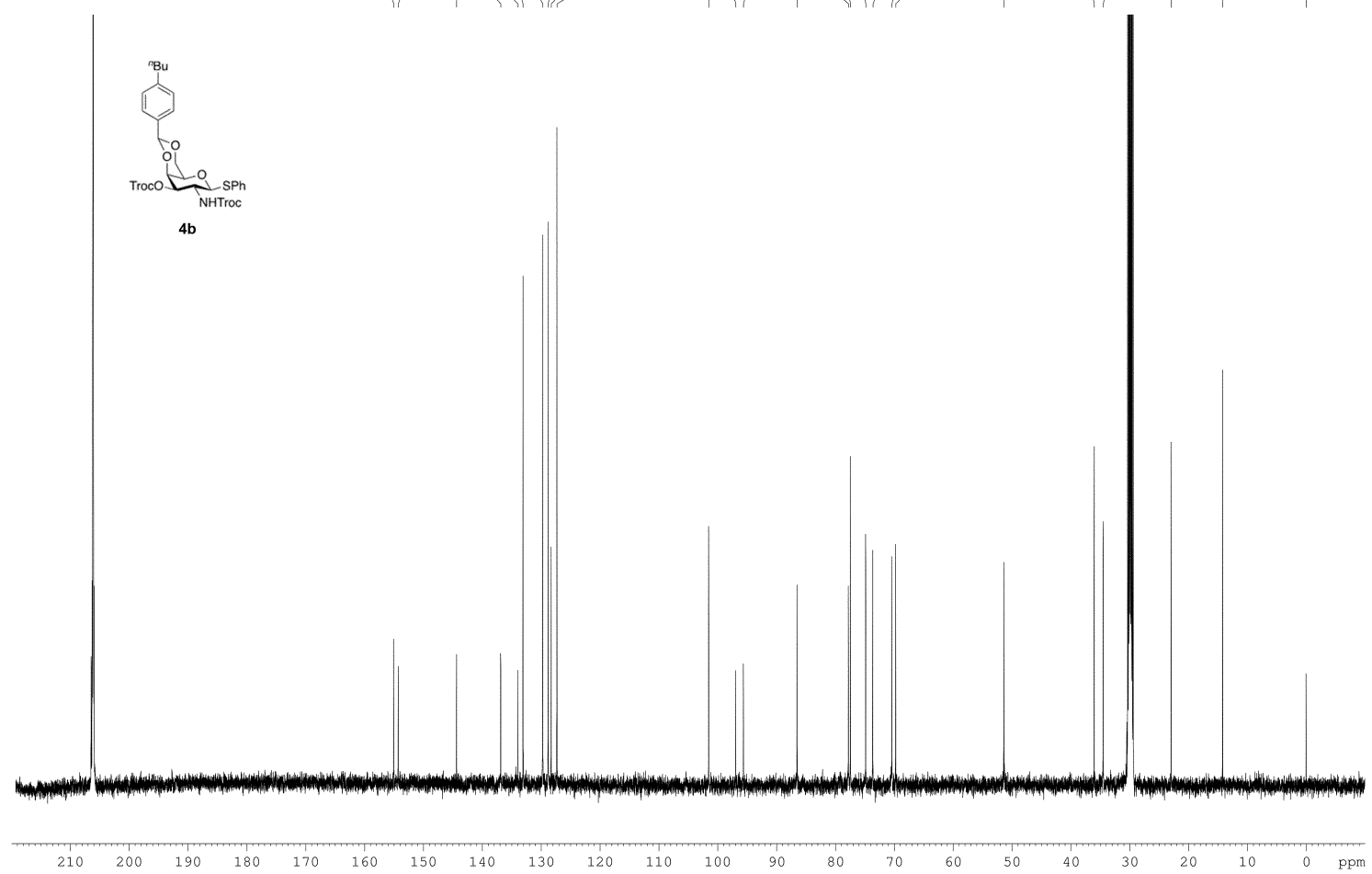



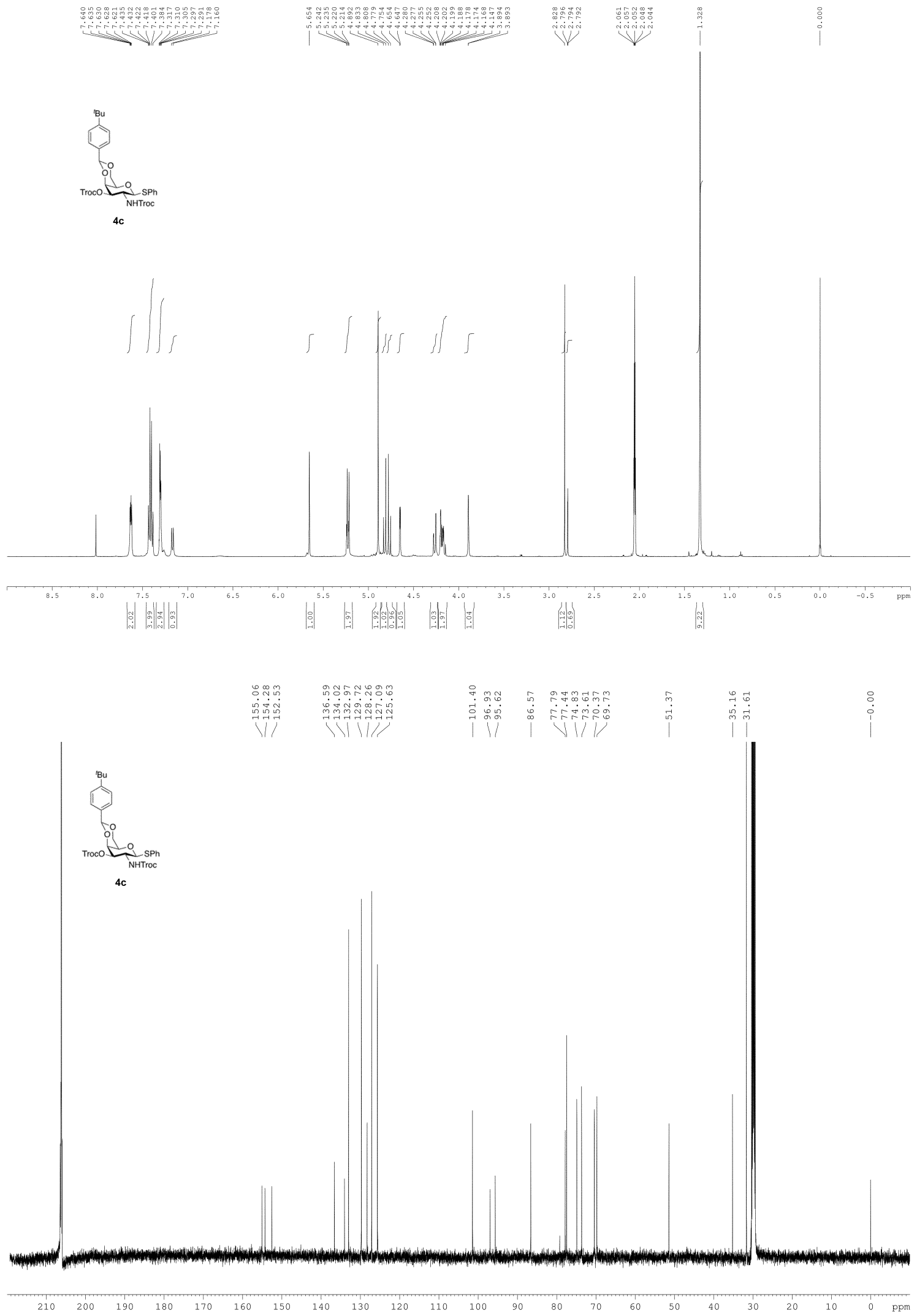


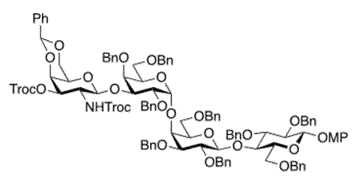

$6 a$

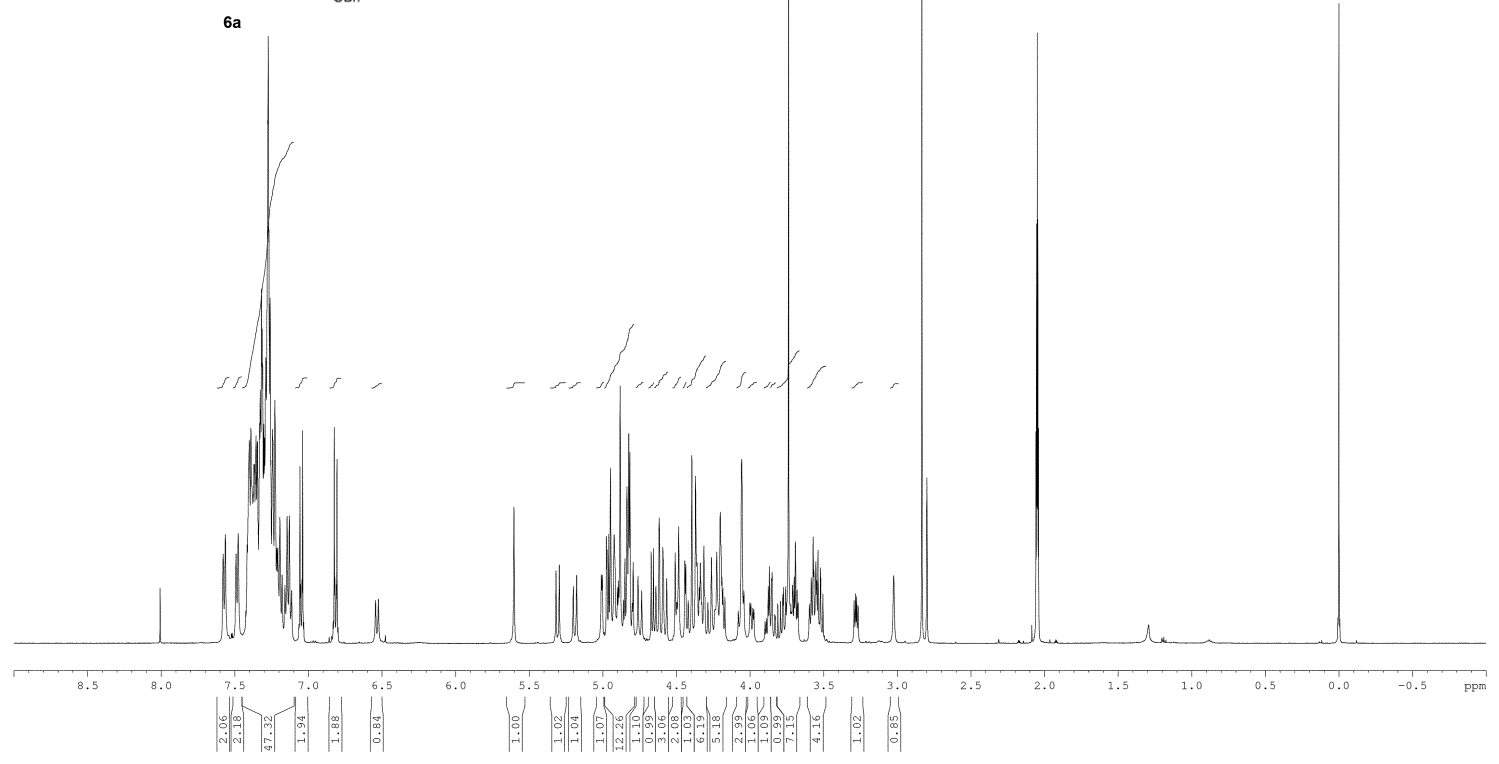

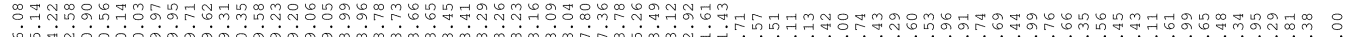

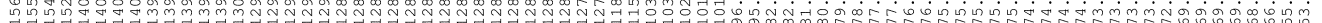

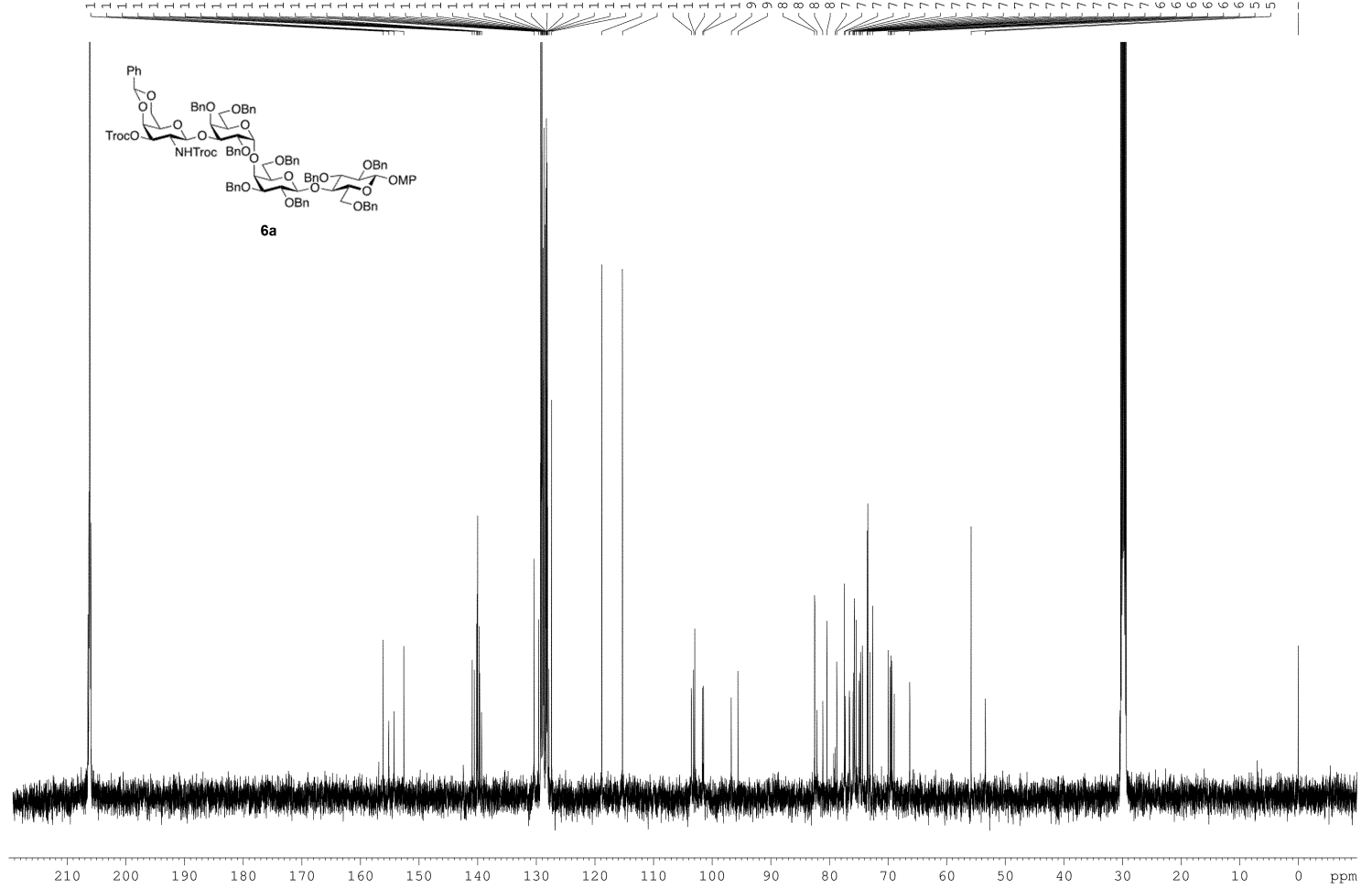




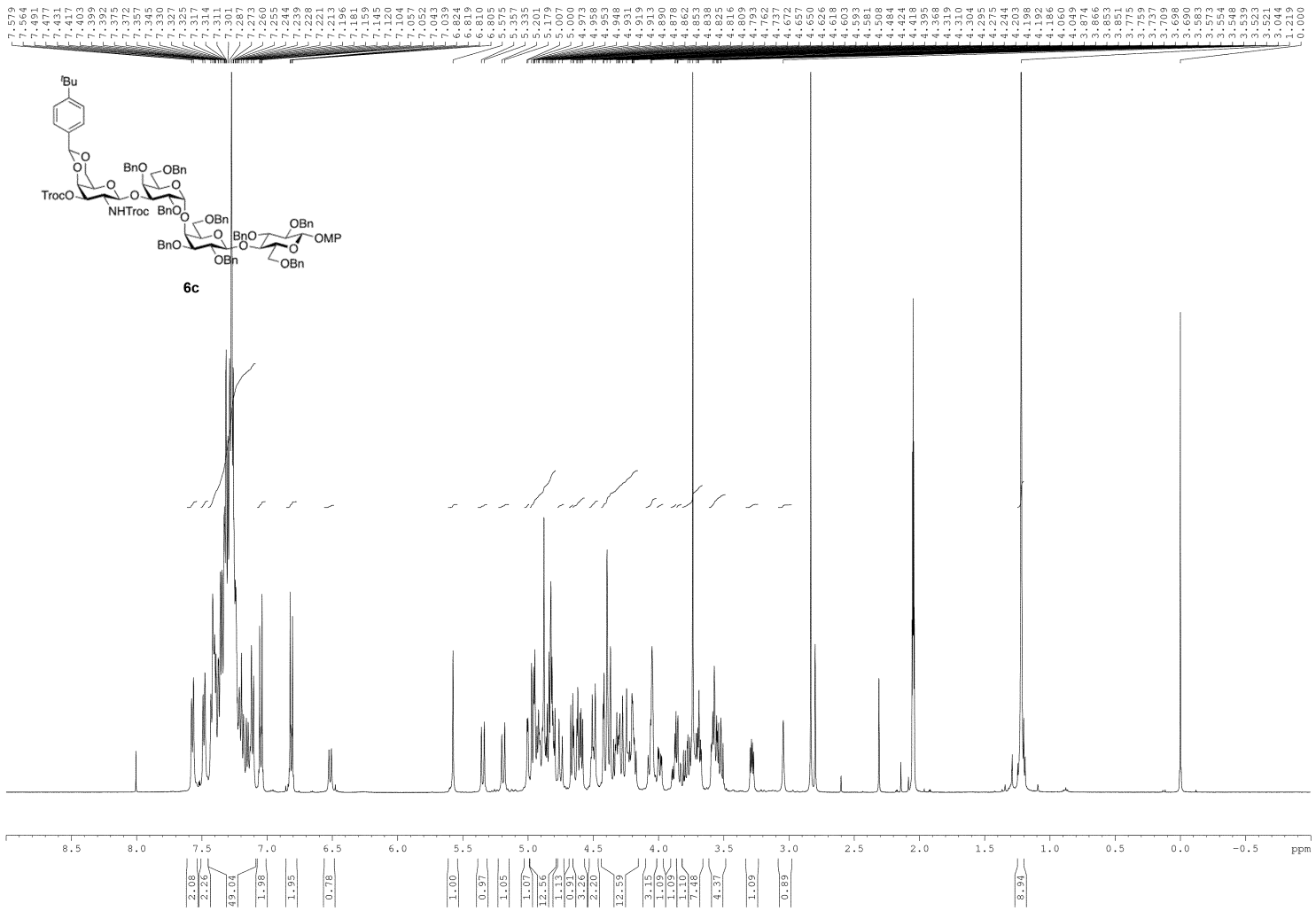

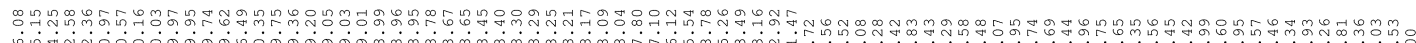

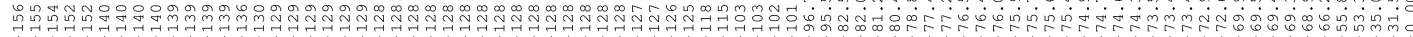

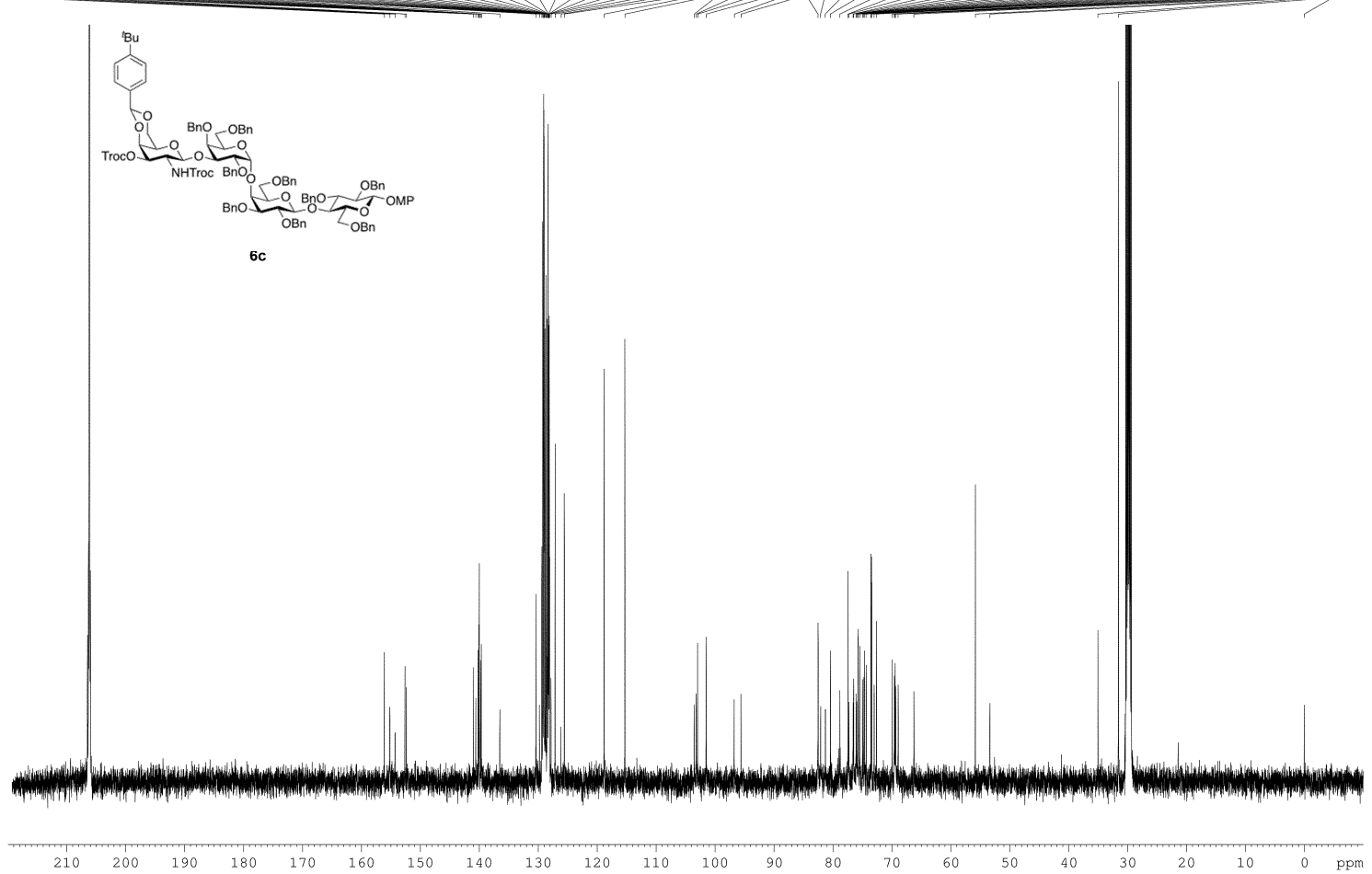




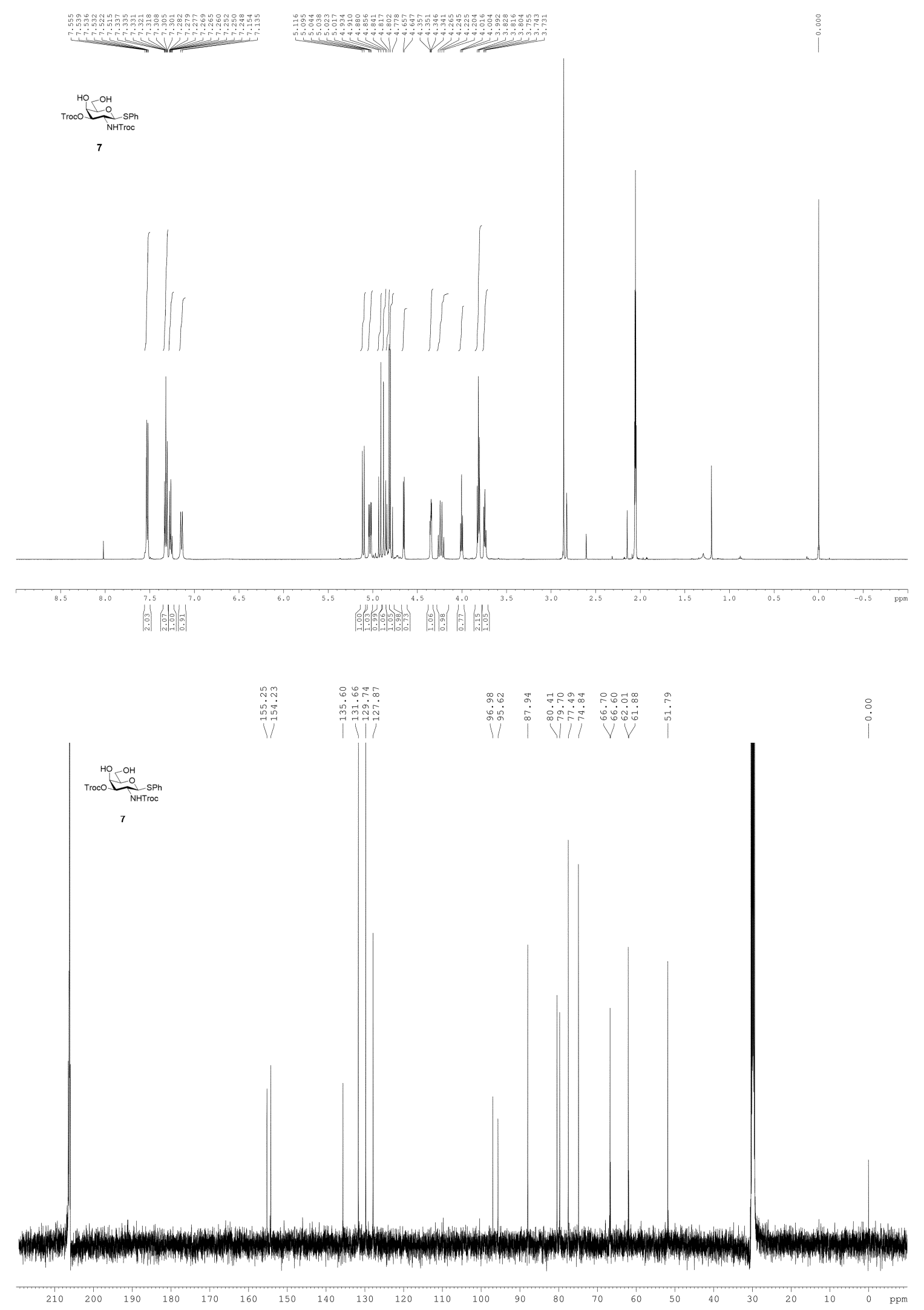



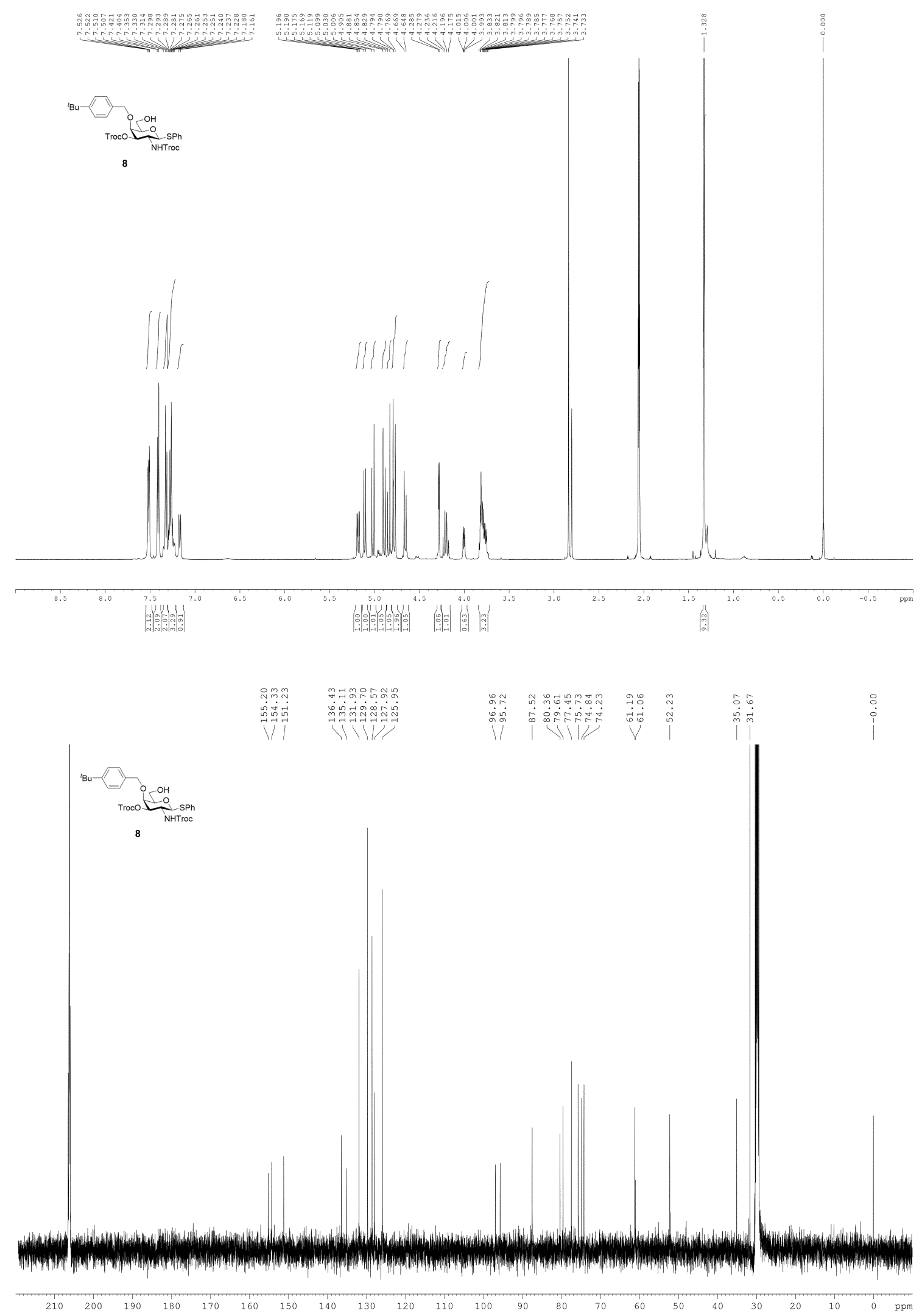


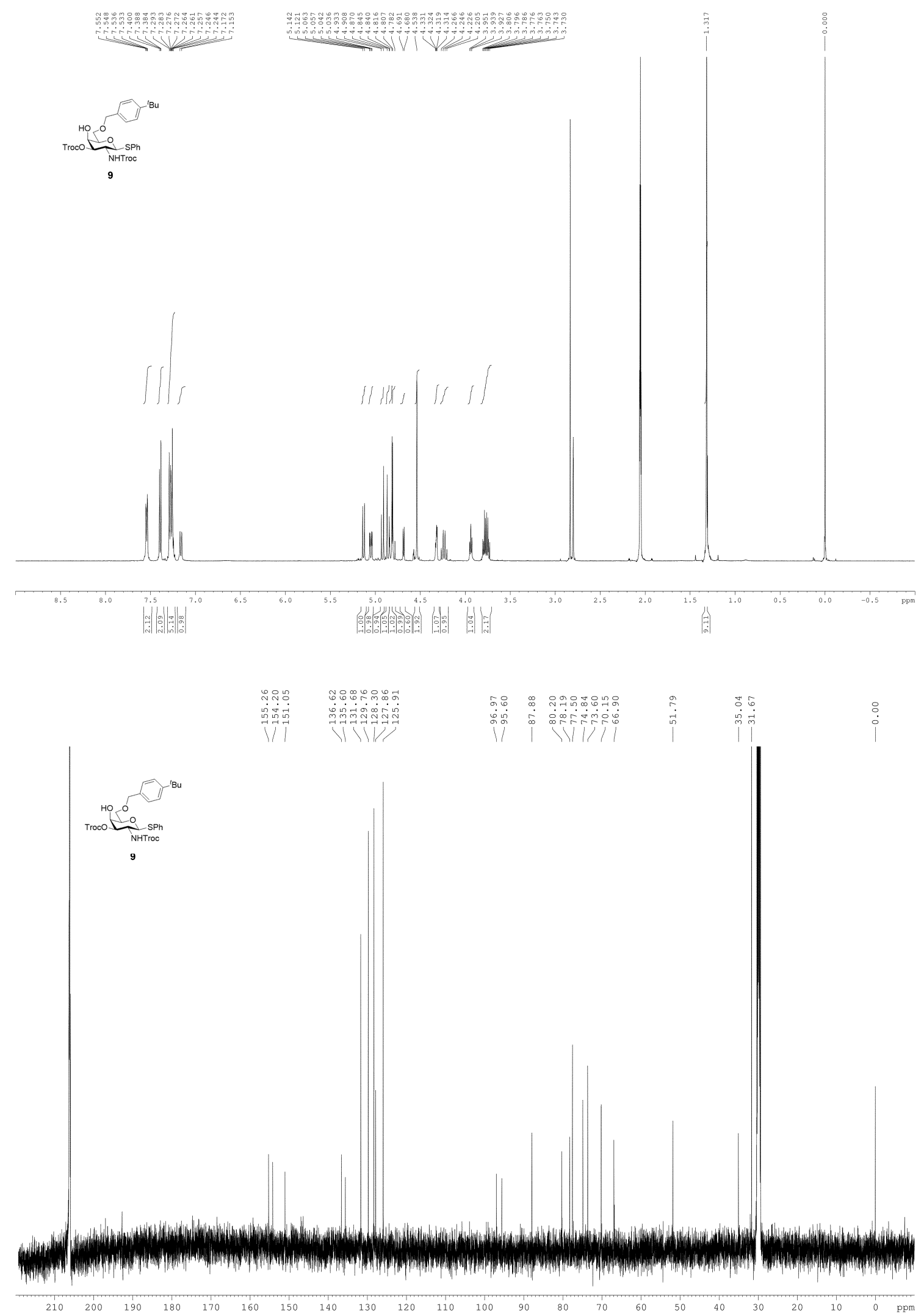



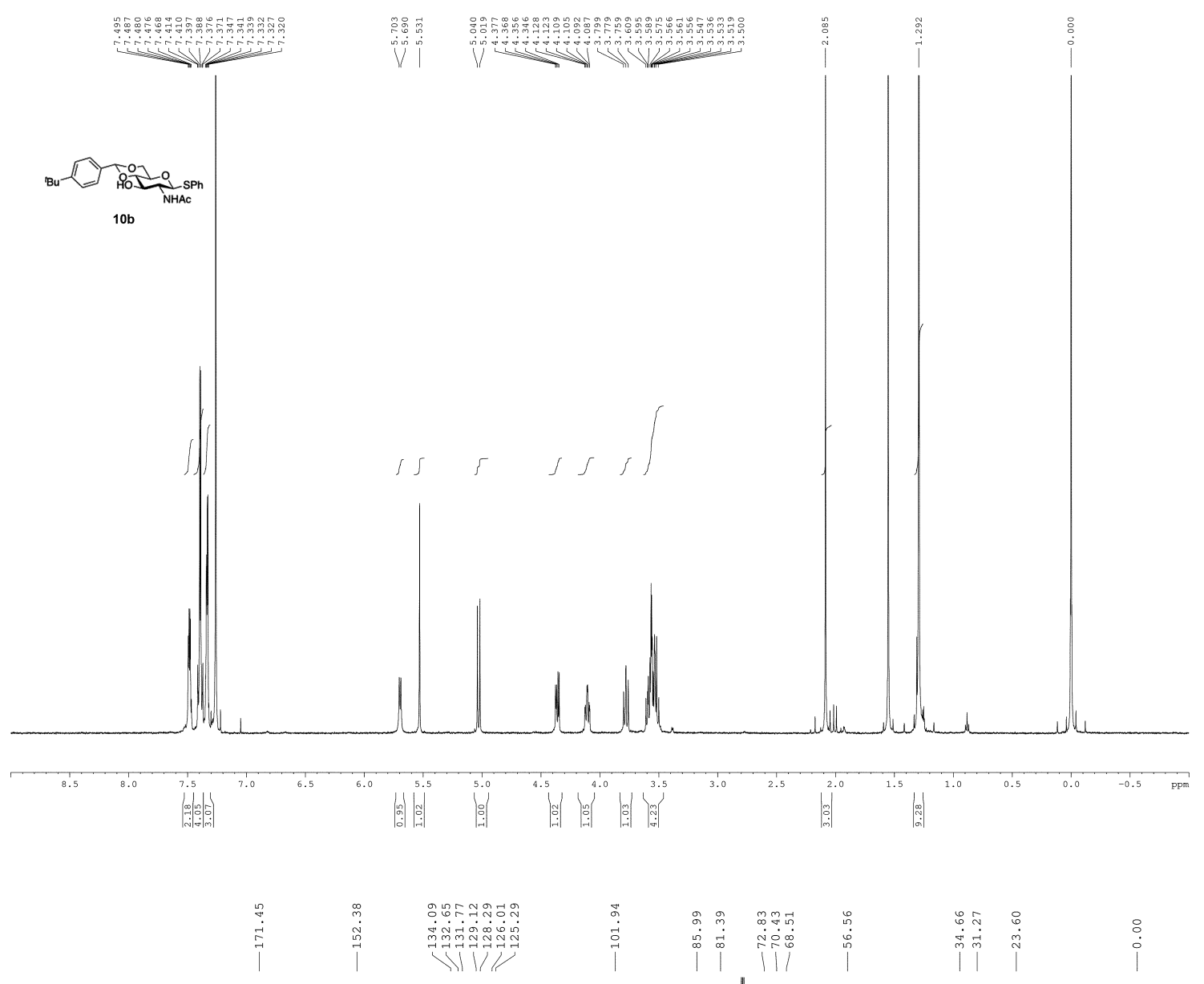

Bu 1 ro

$10 \mathrm{~b}$

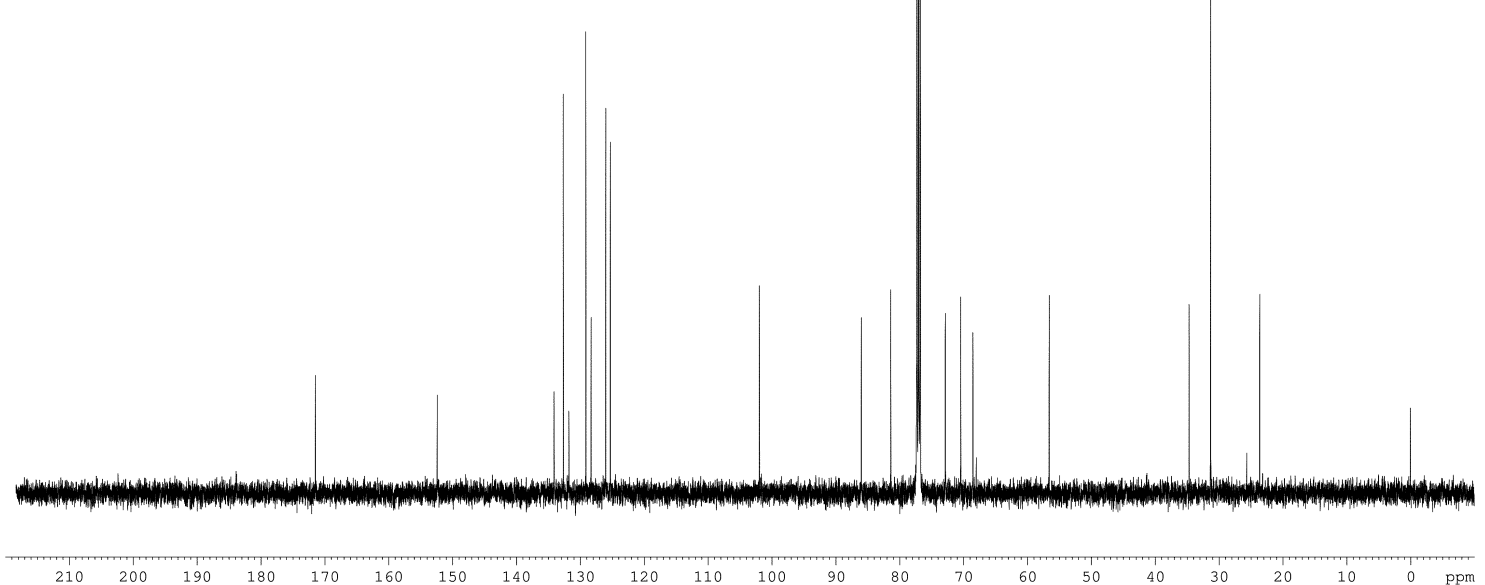




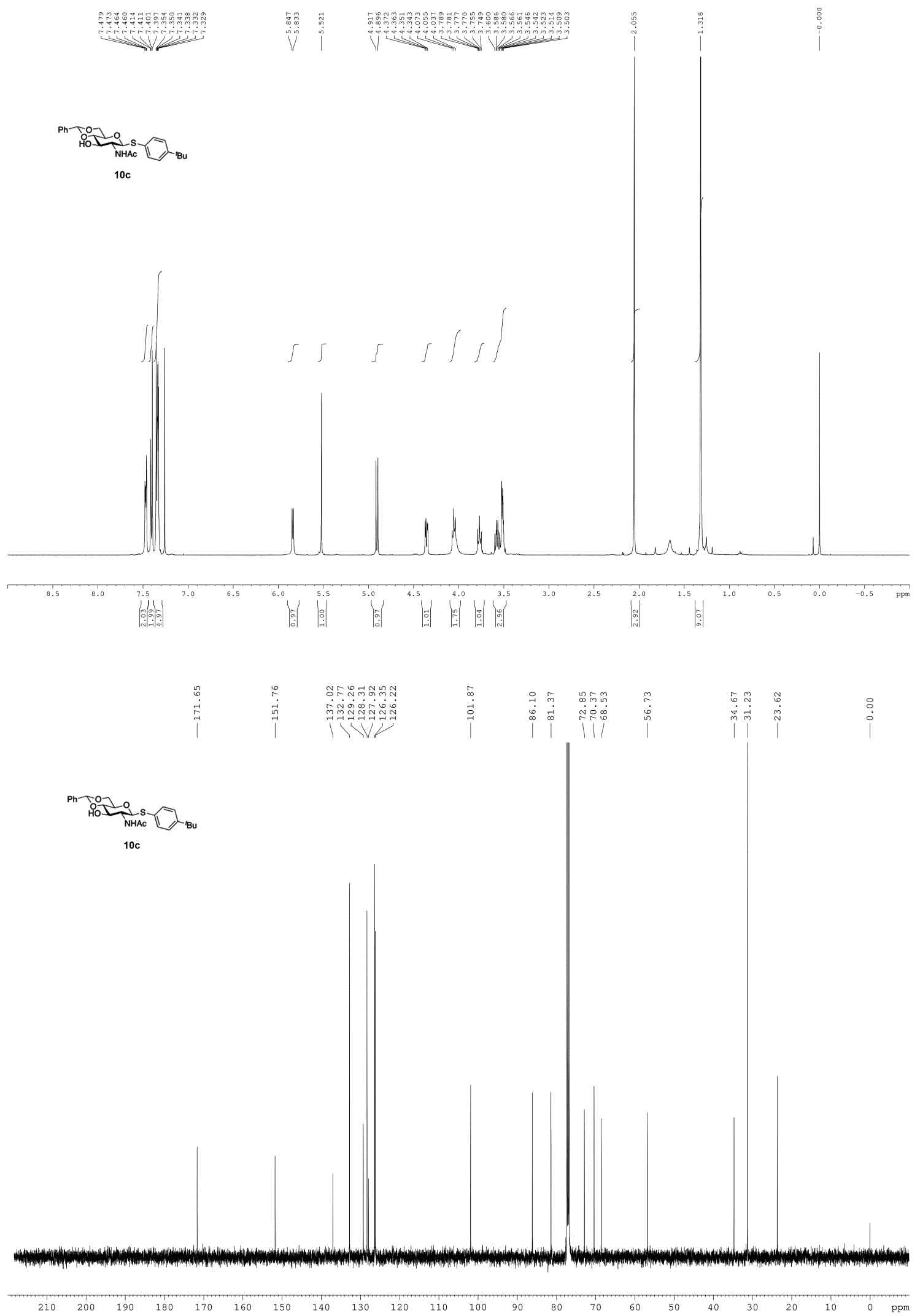




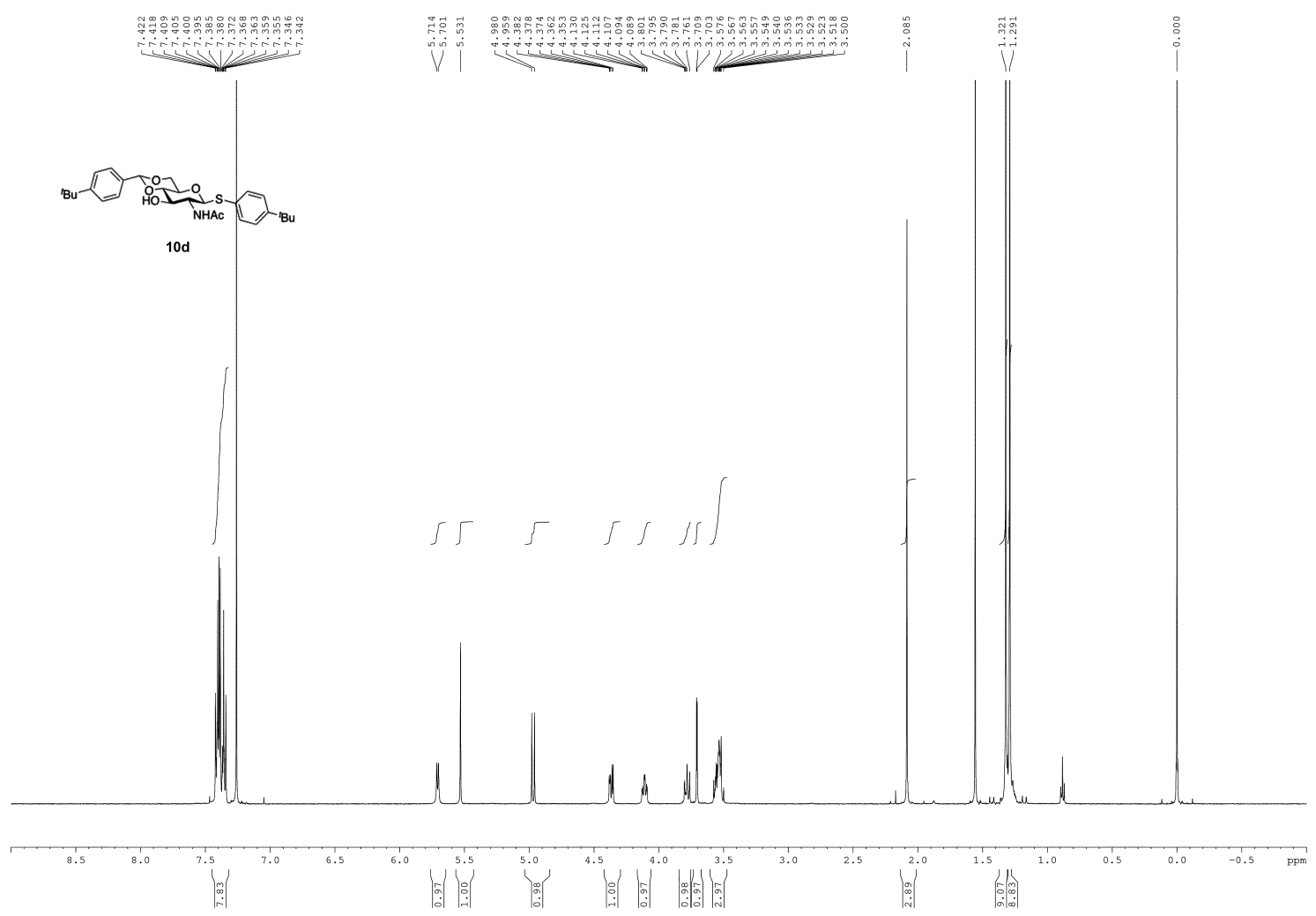

|

100

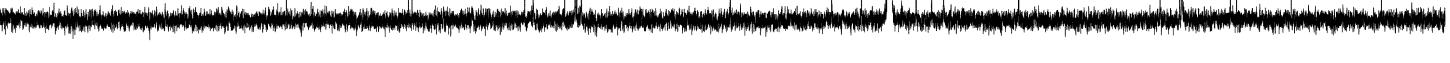

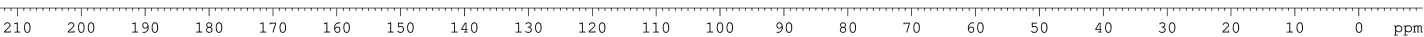




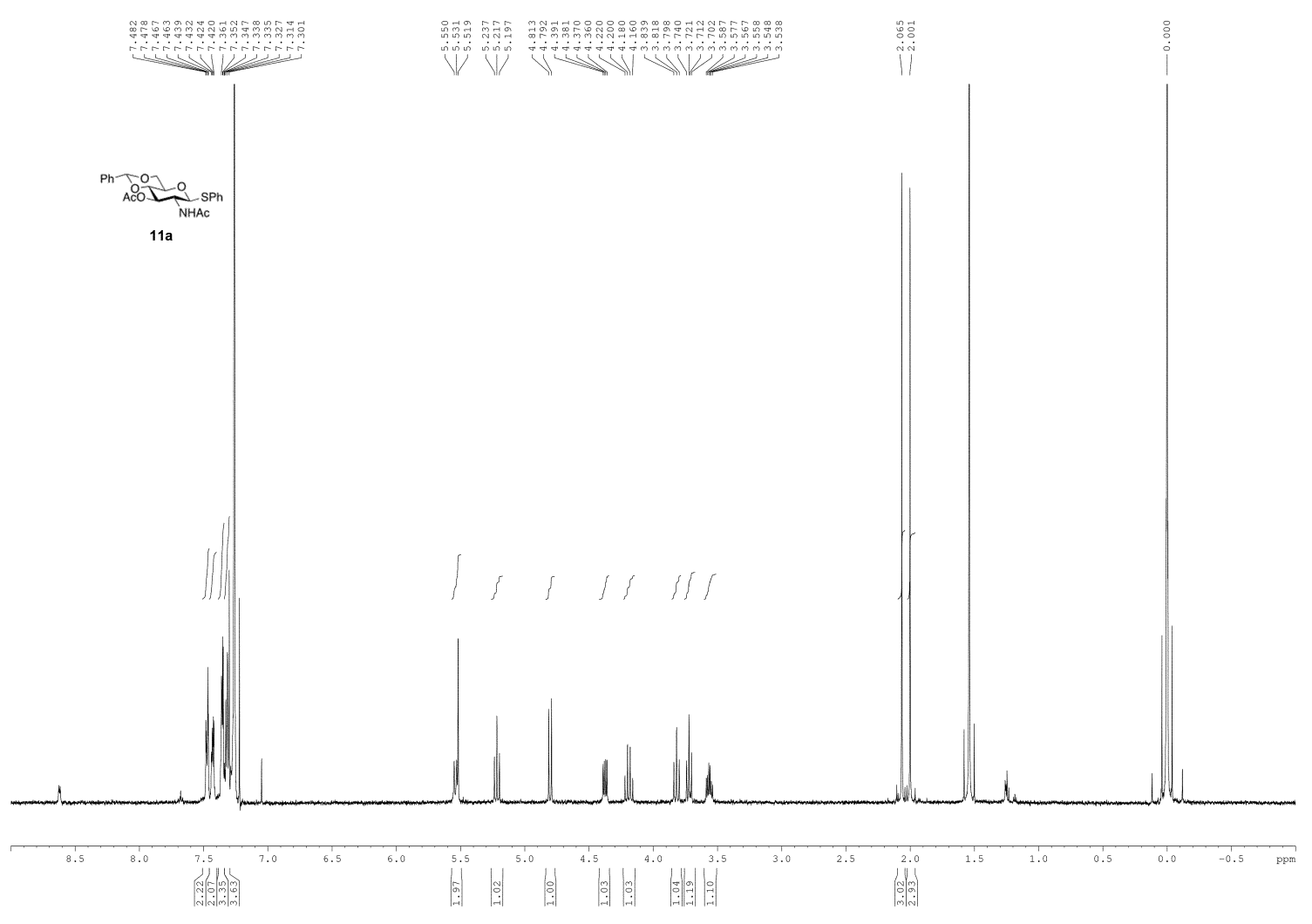




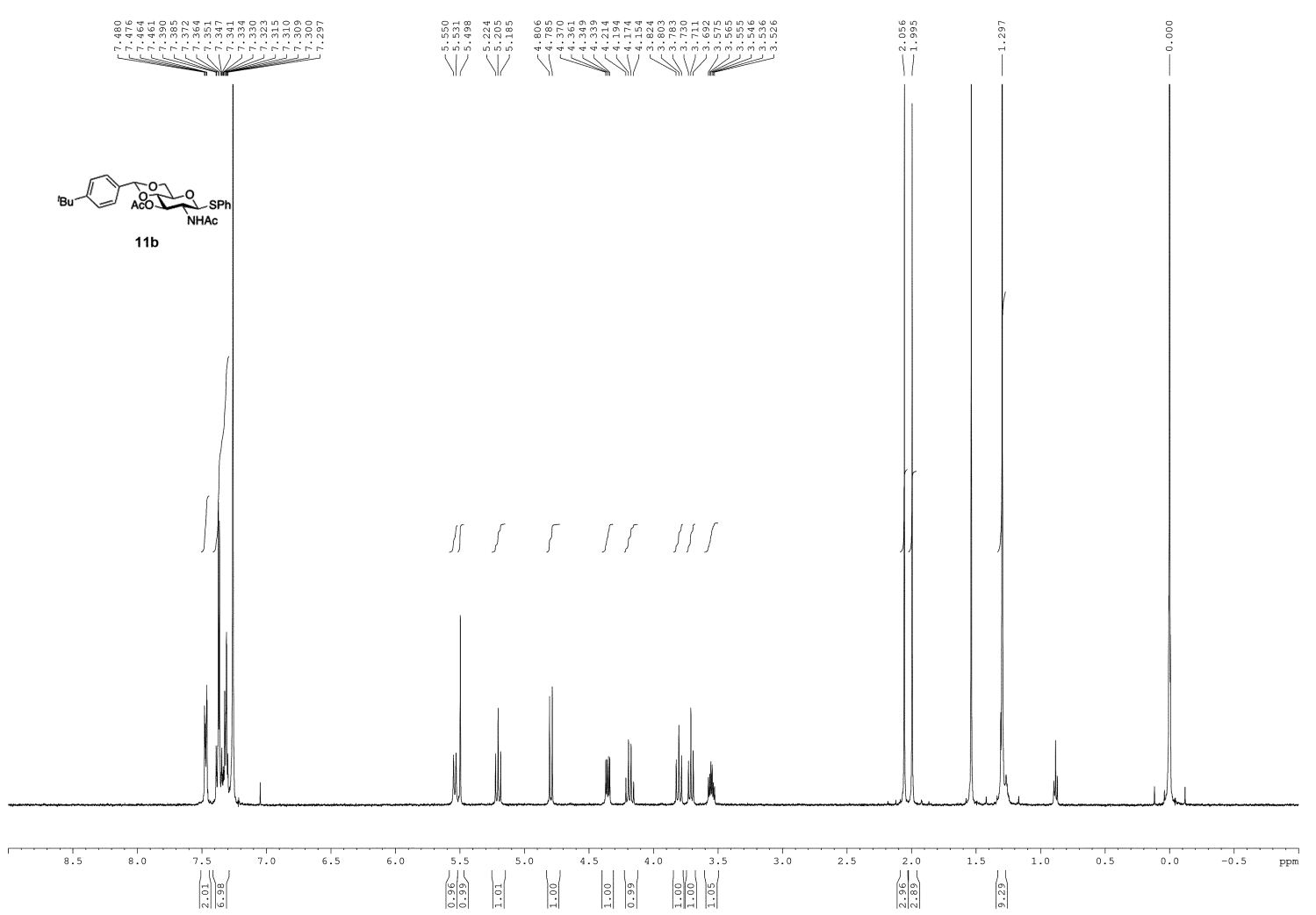

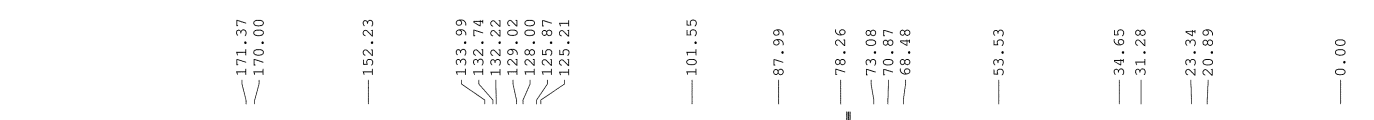

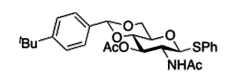

11b

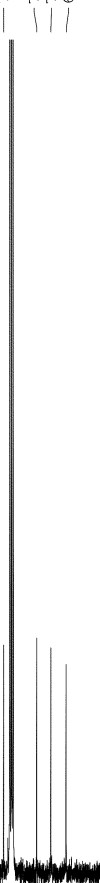

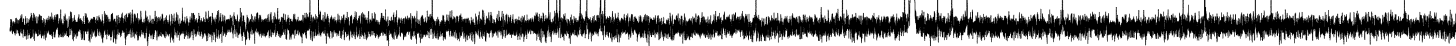



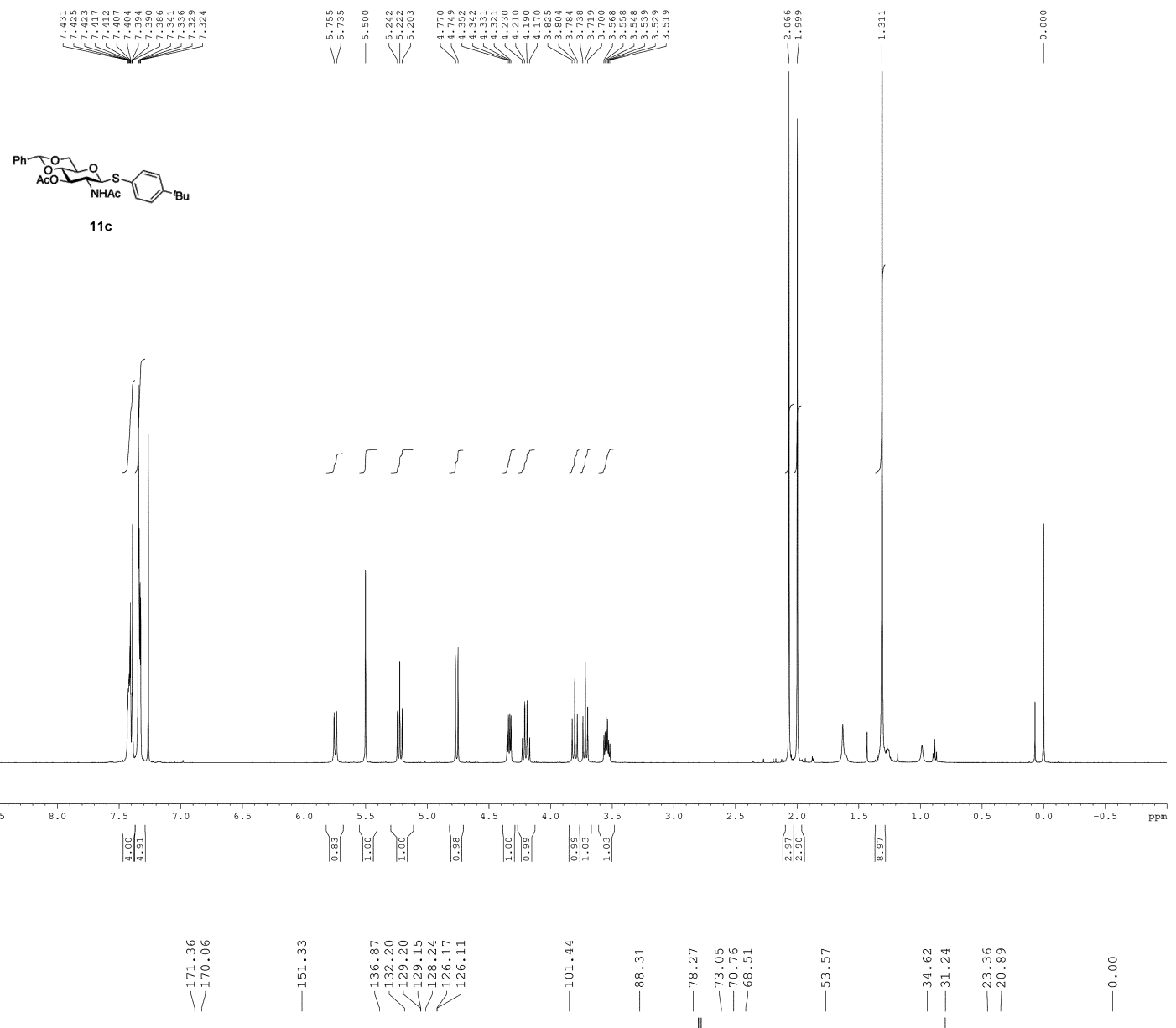

ACO

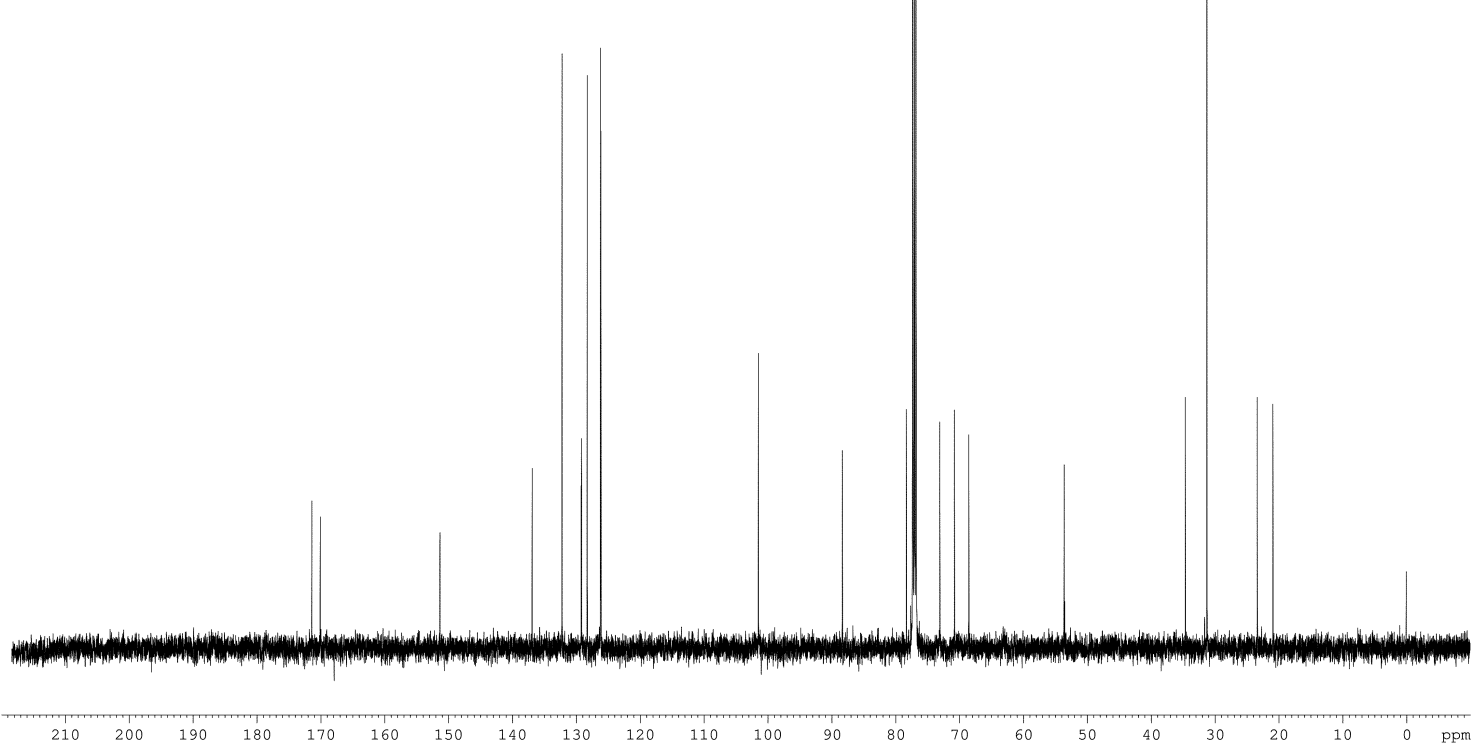




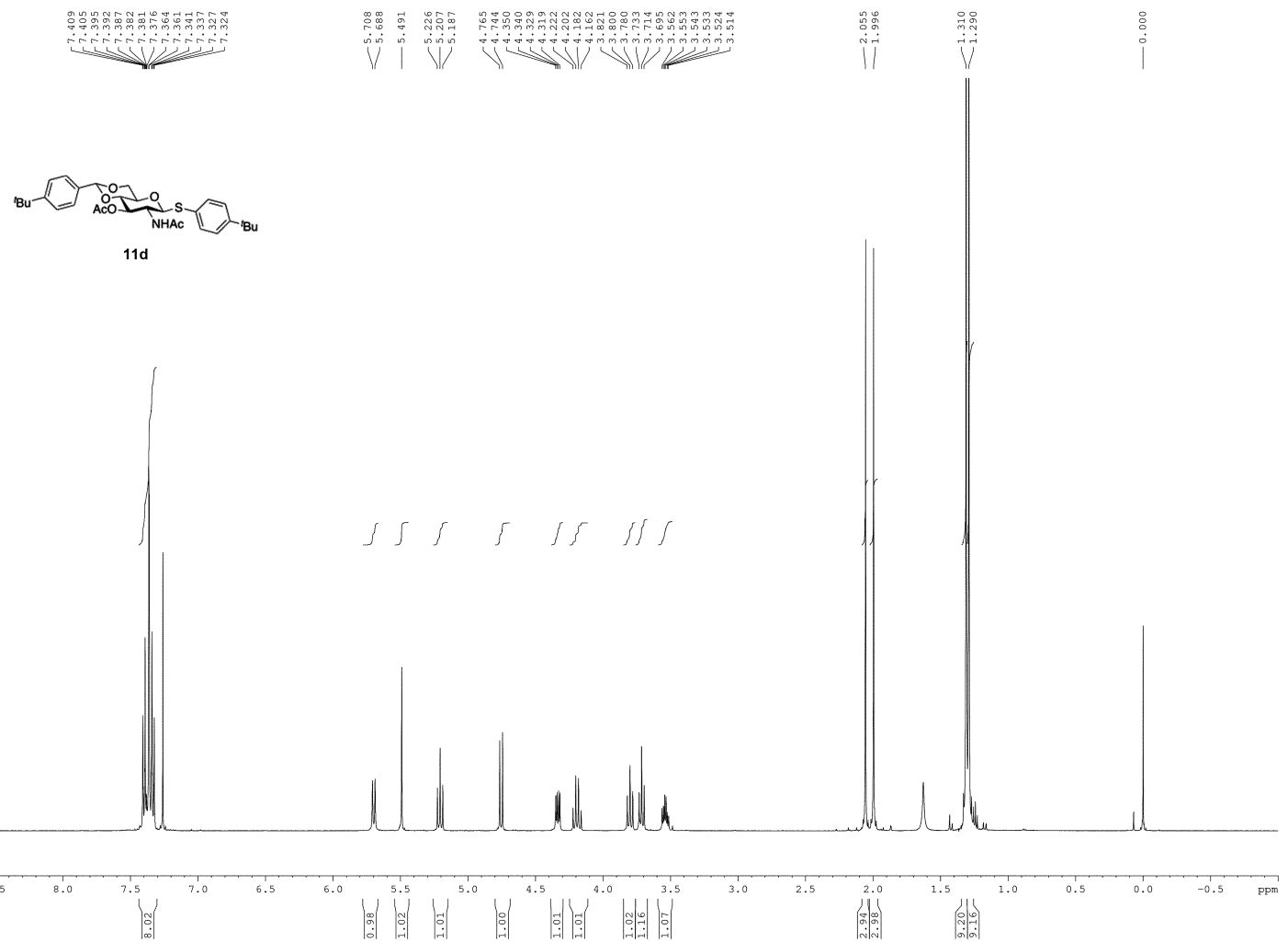

Vic

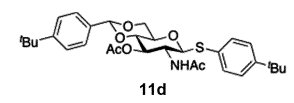

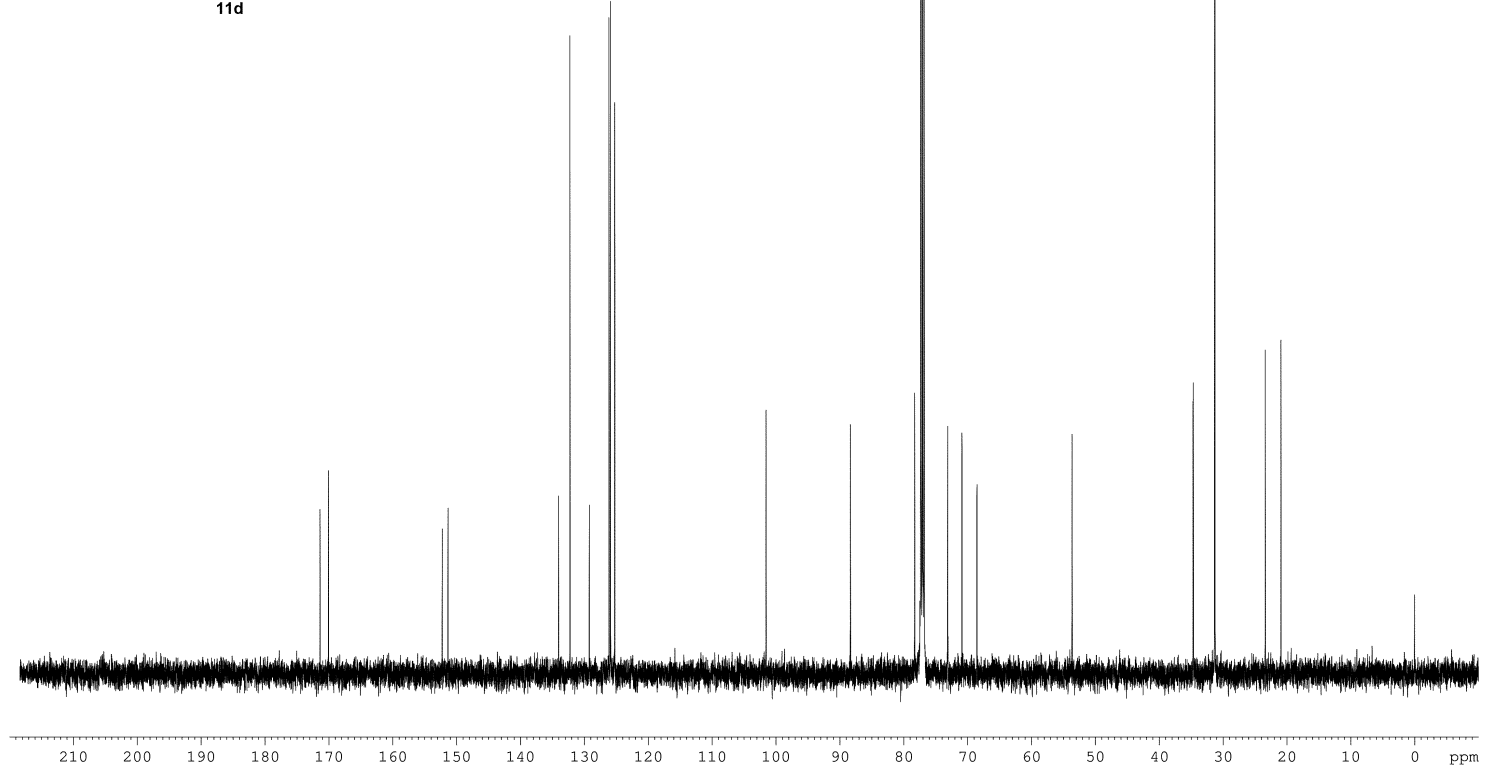



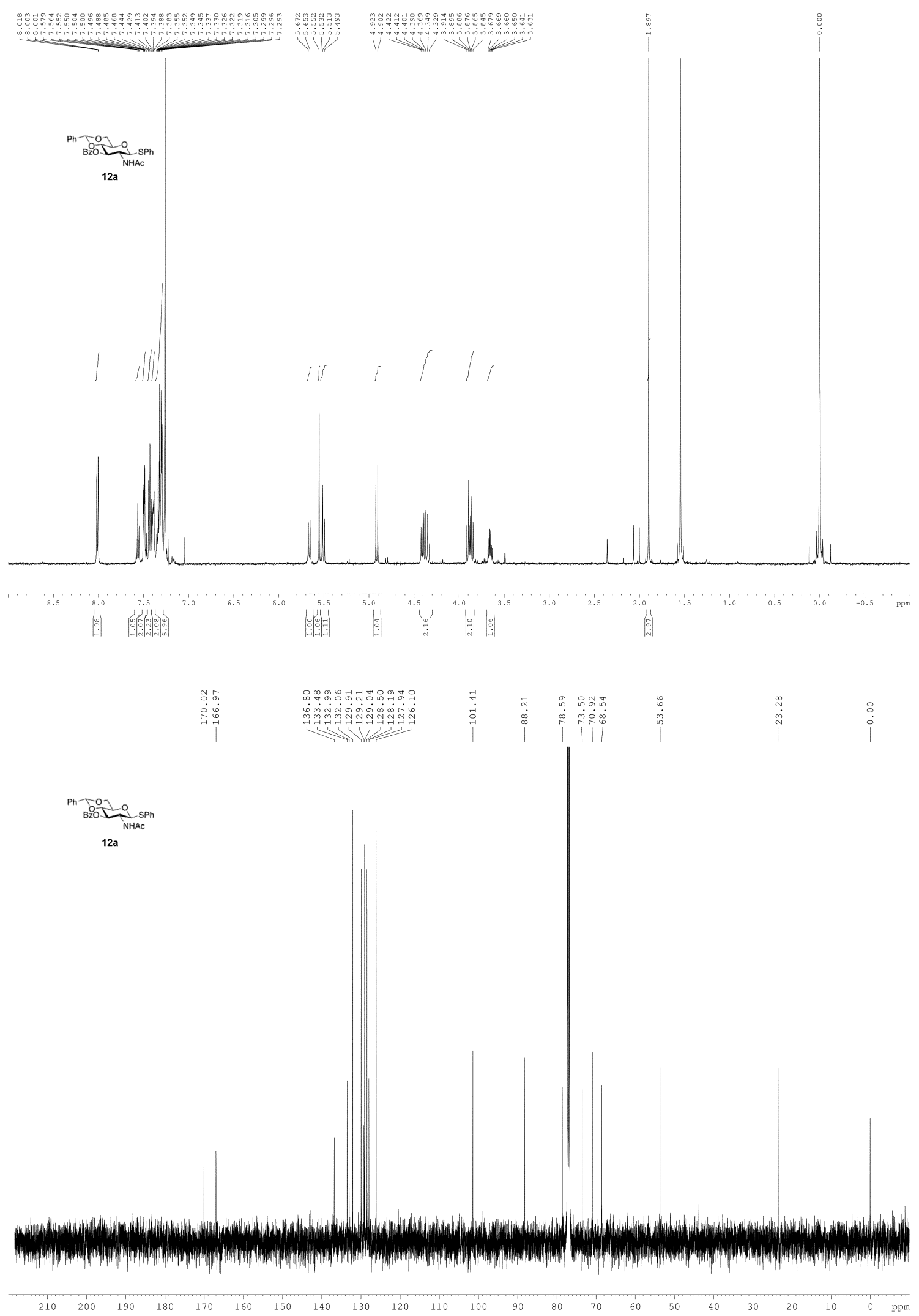

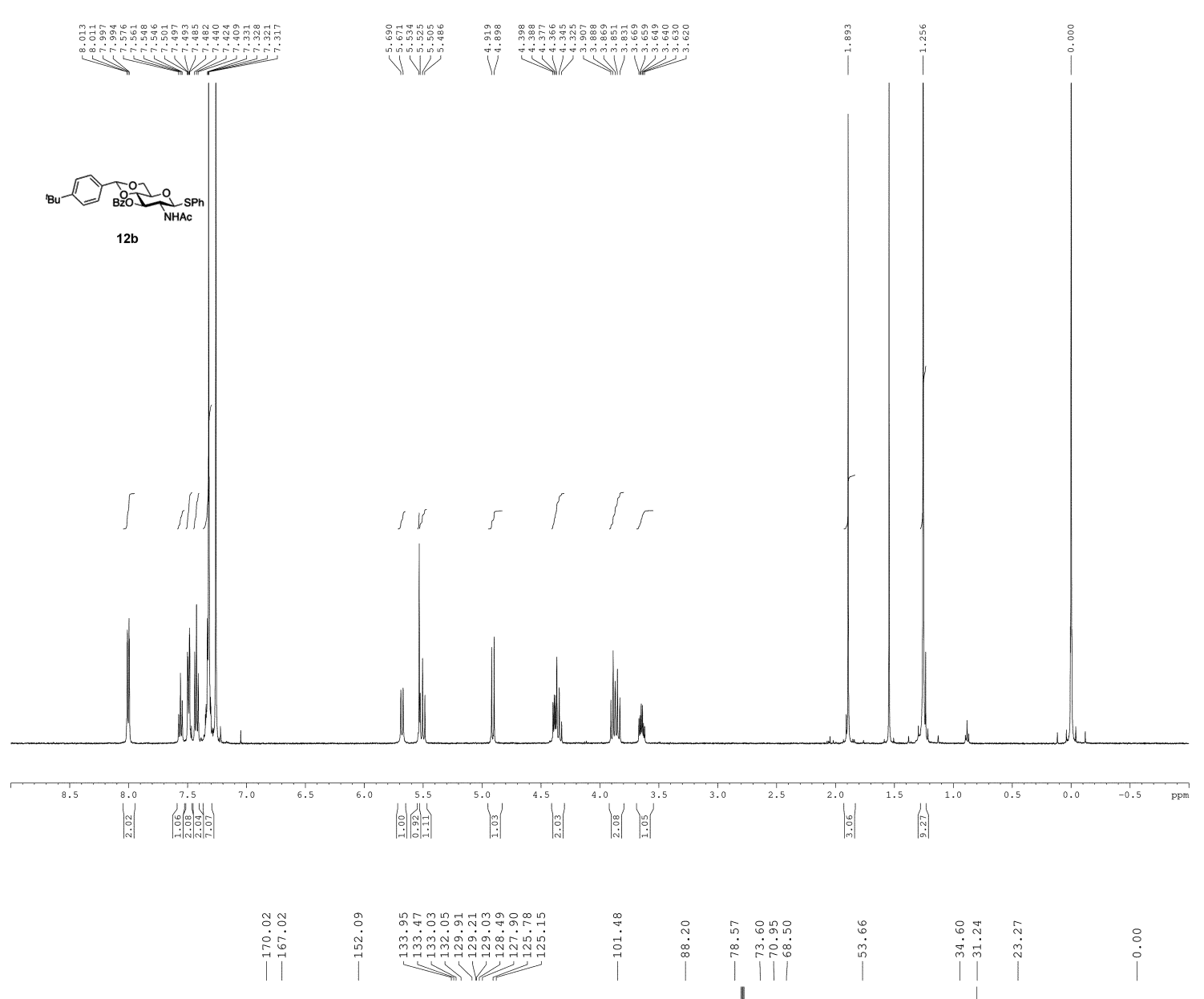

Bu- 1.

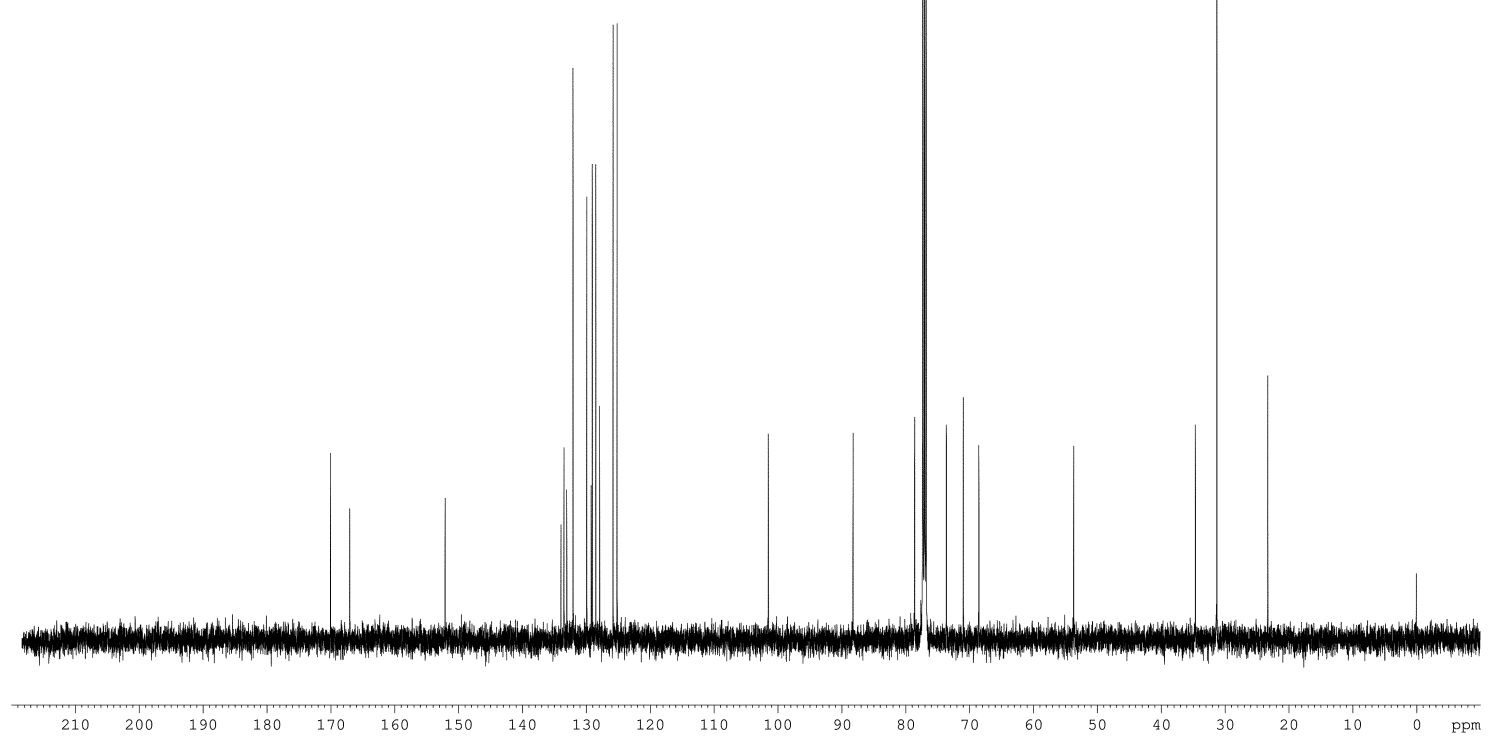



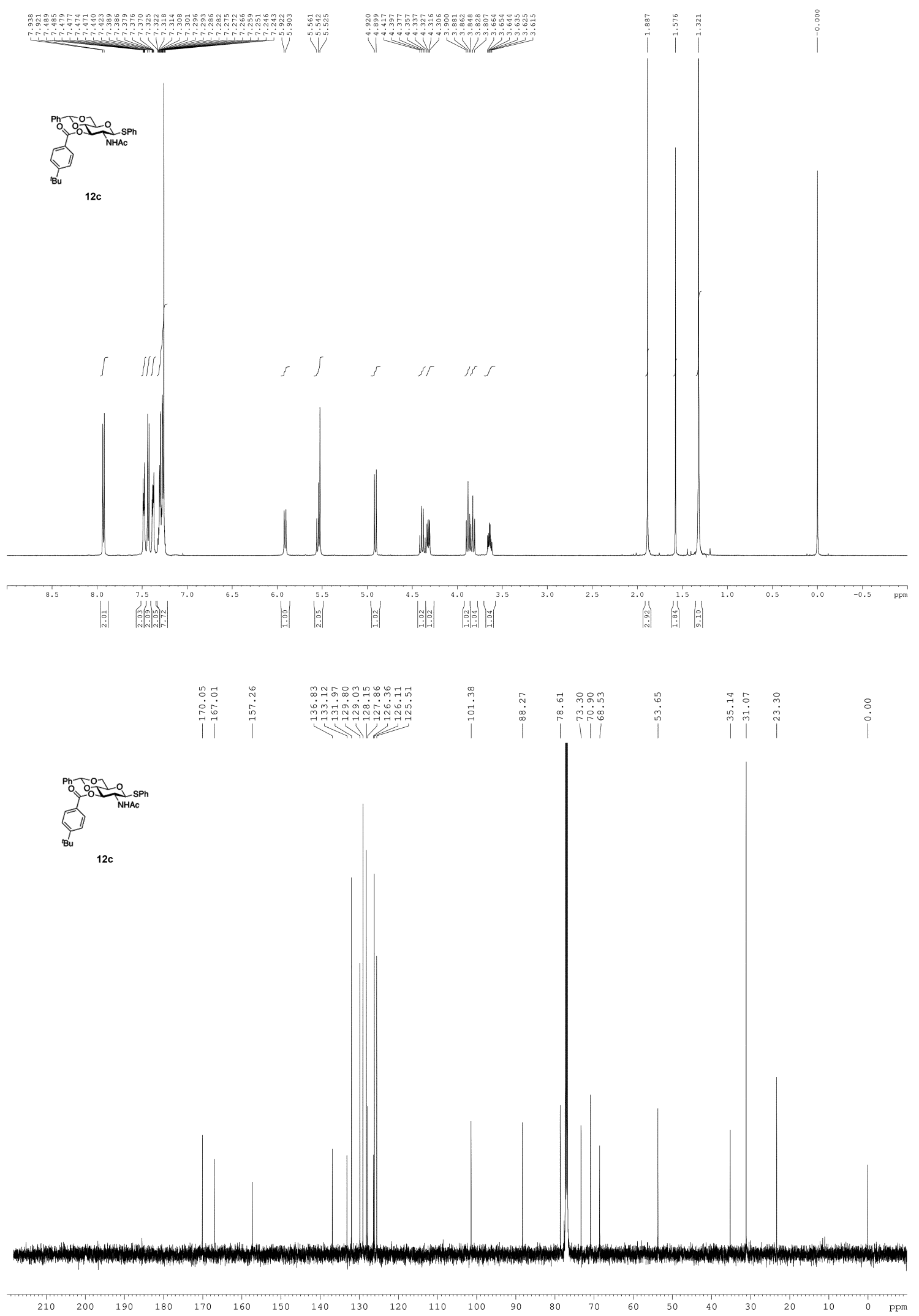

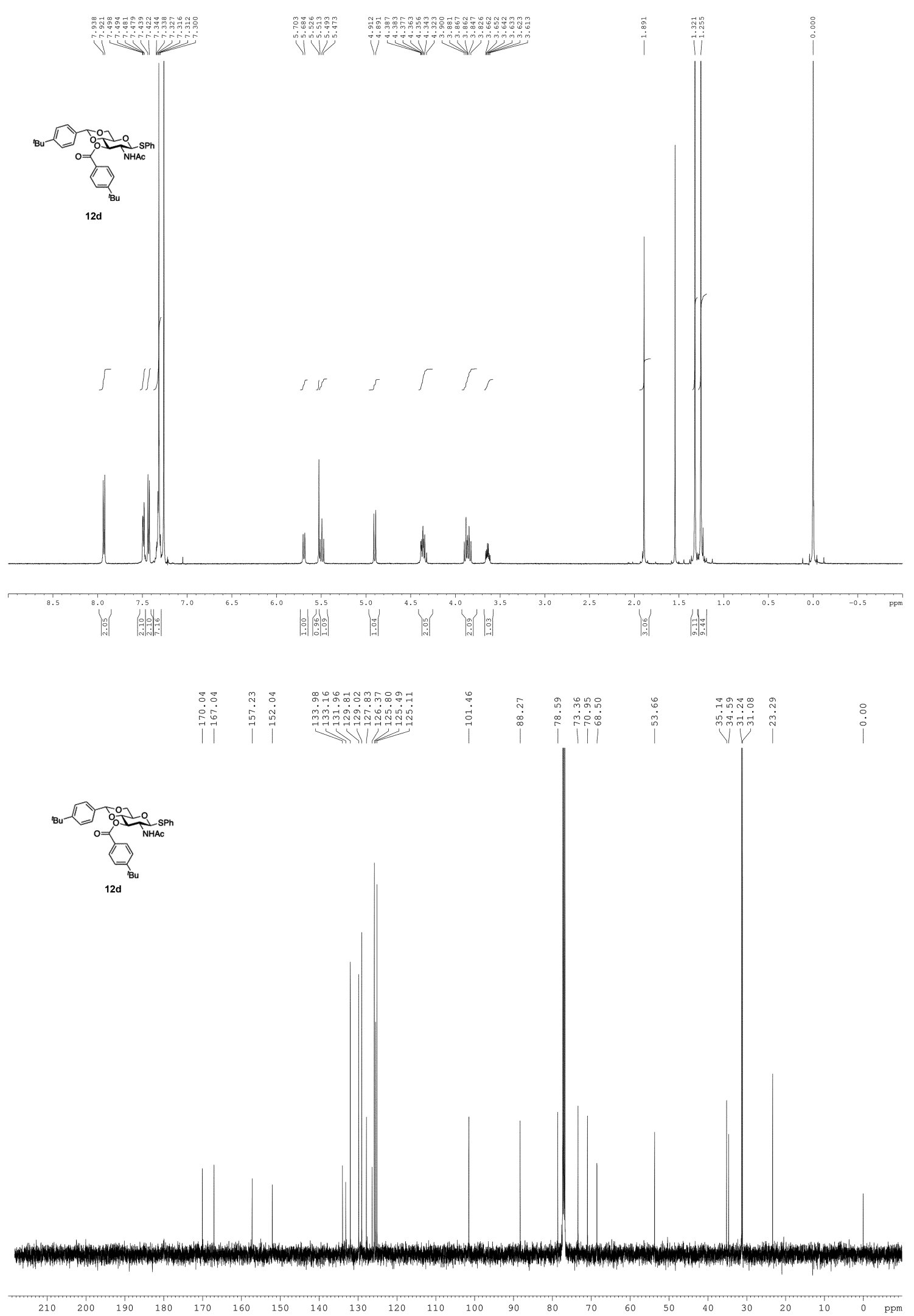


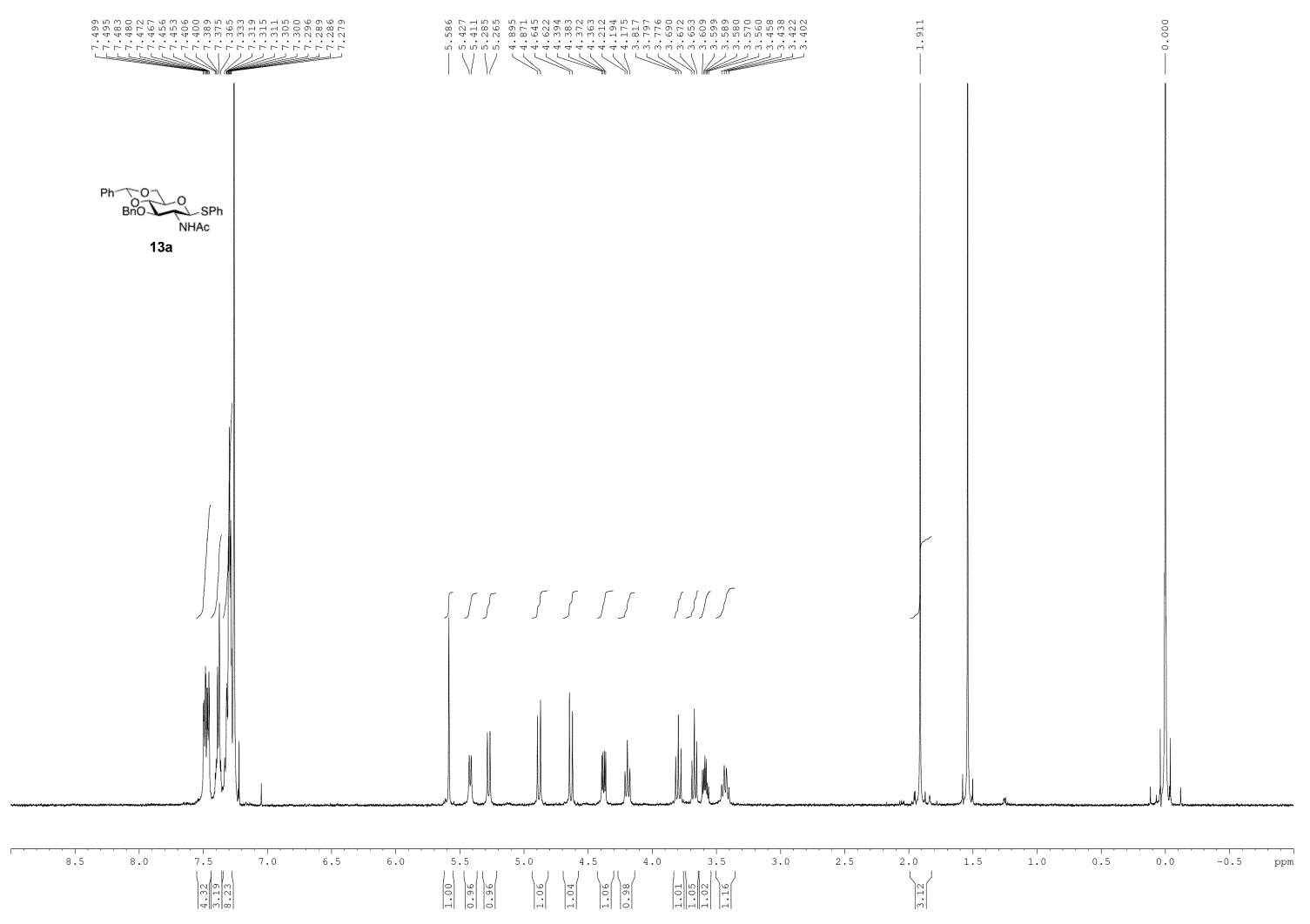



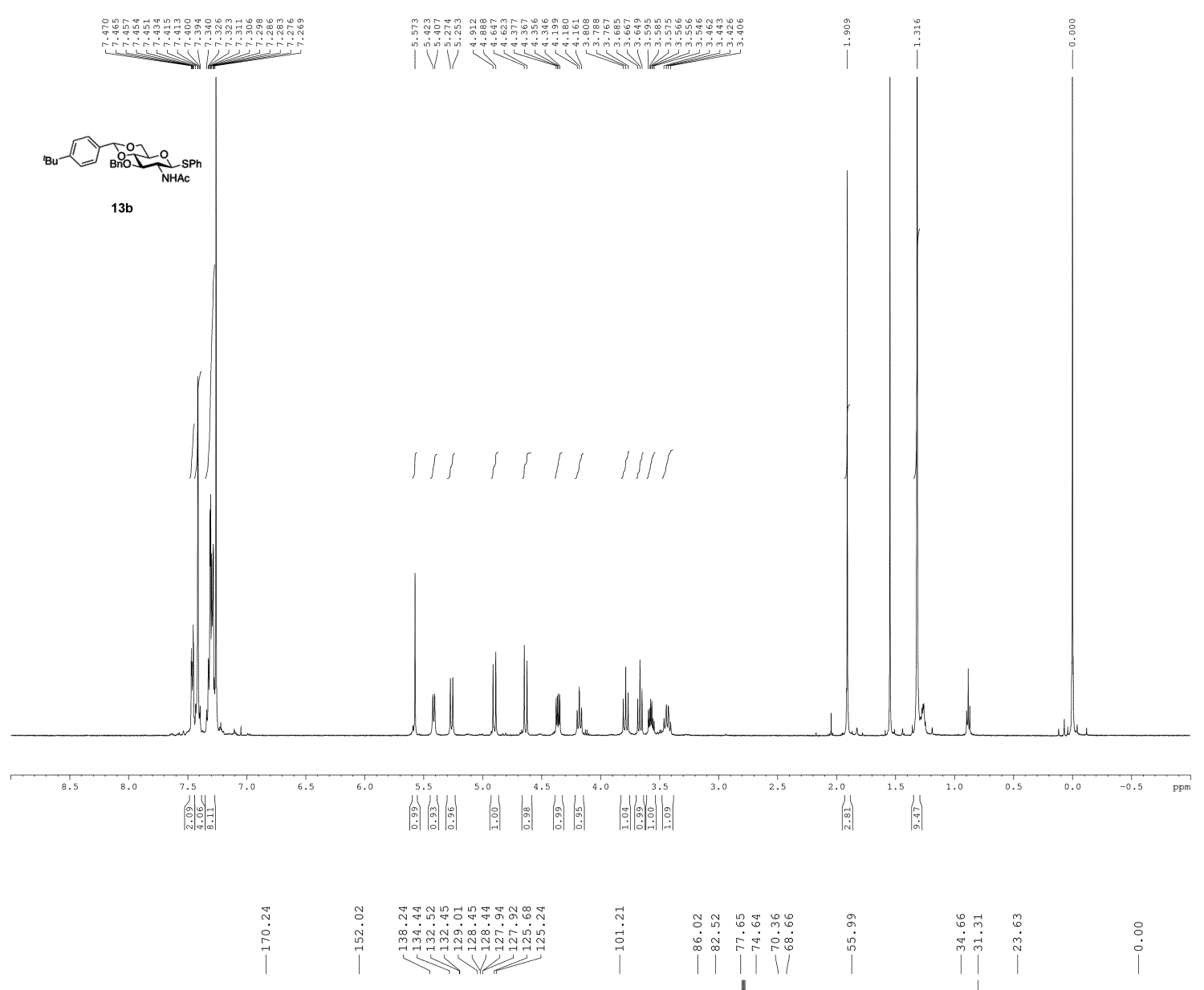

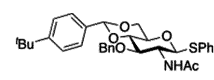

13b

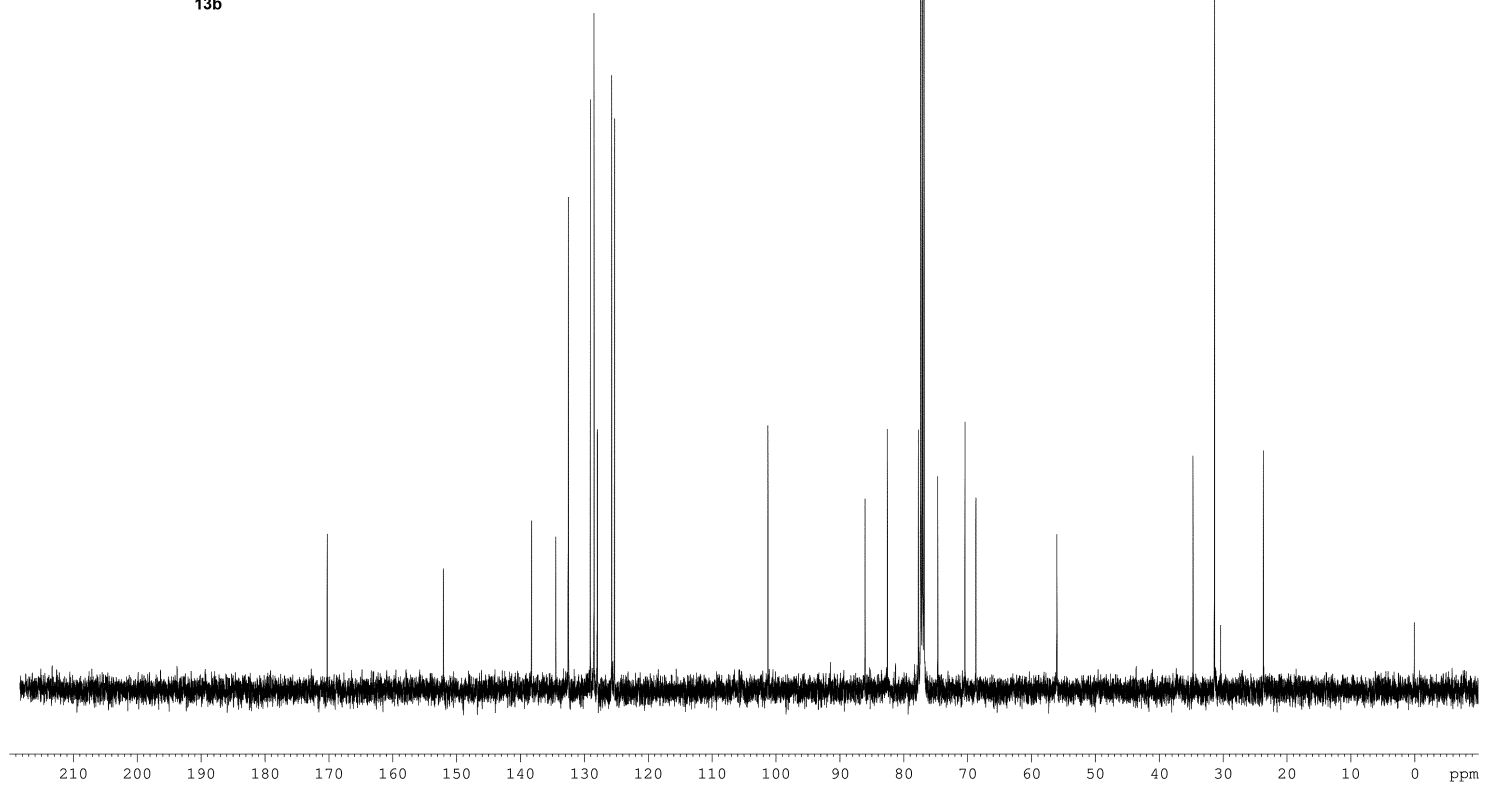



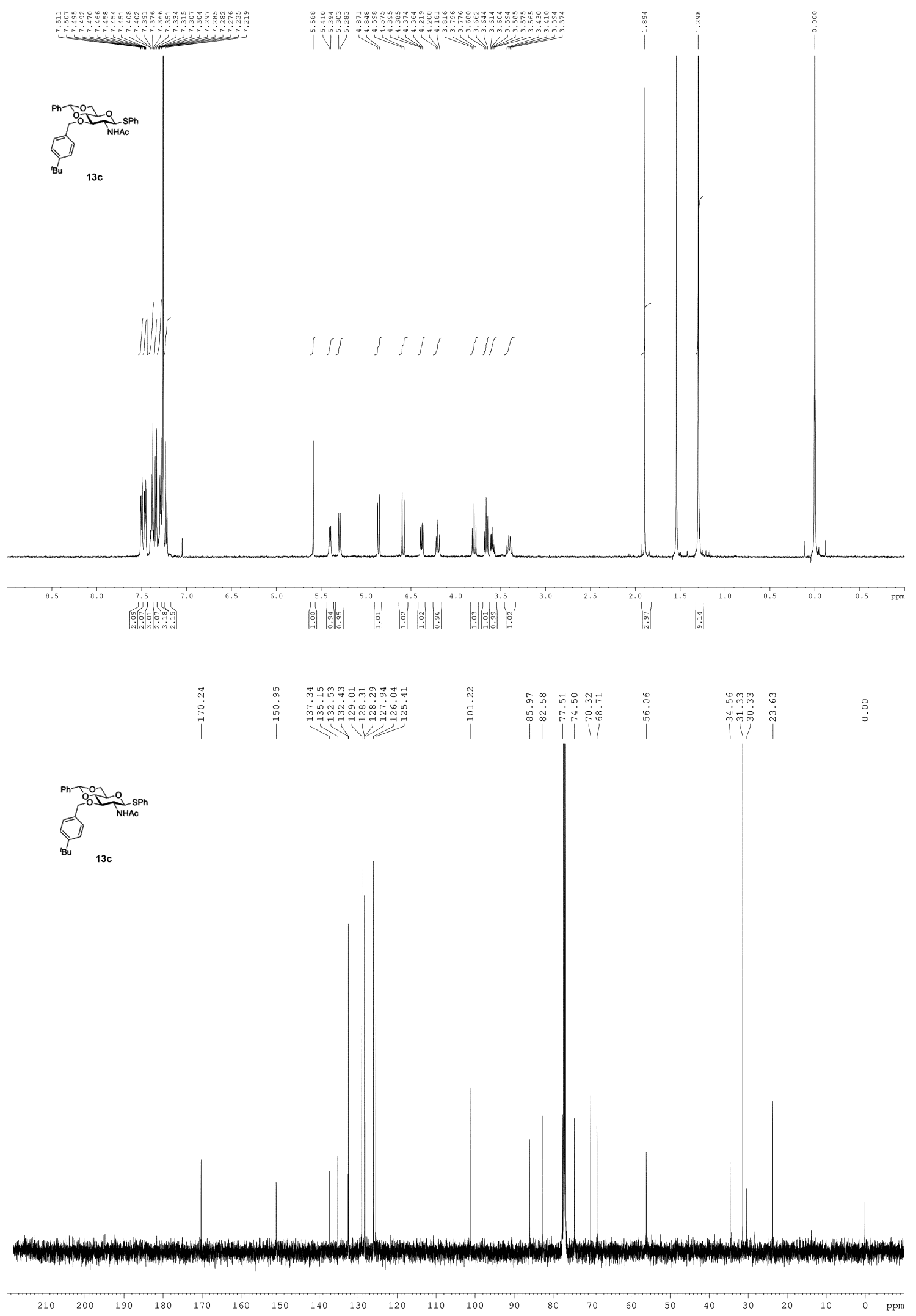

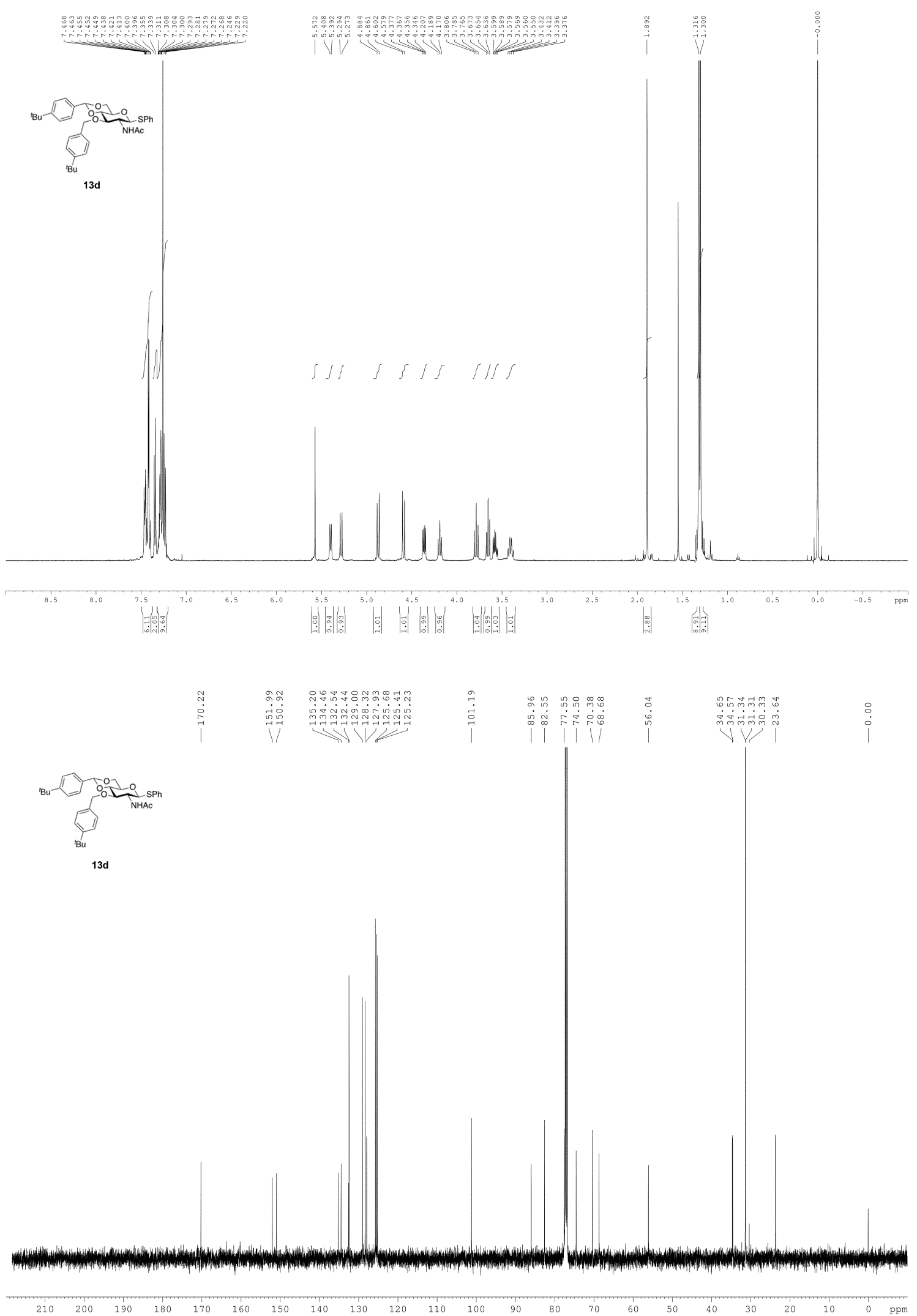


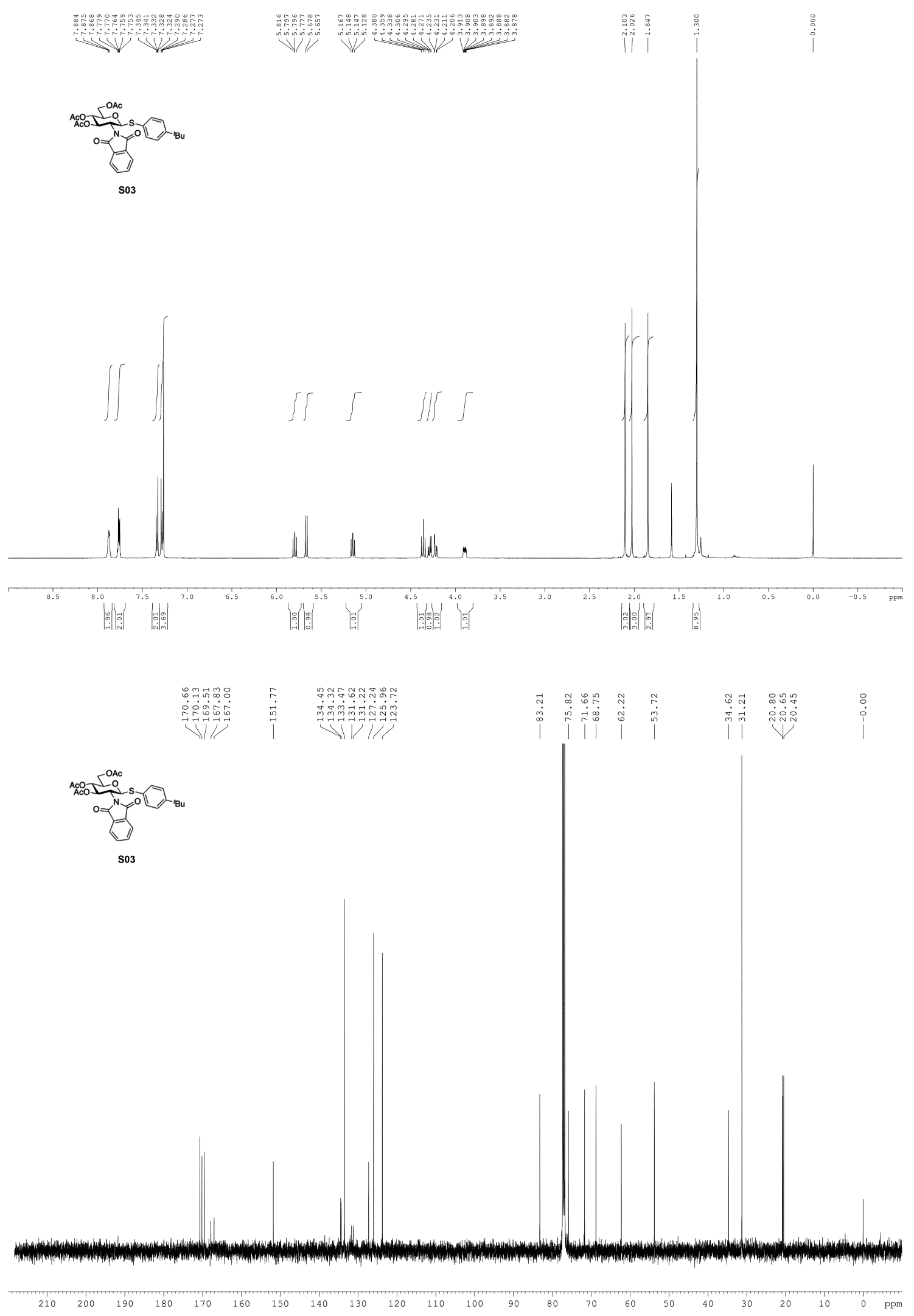




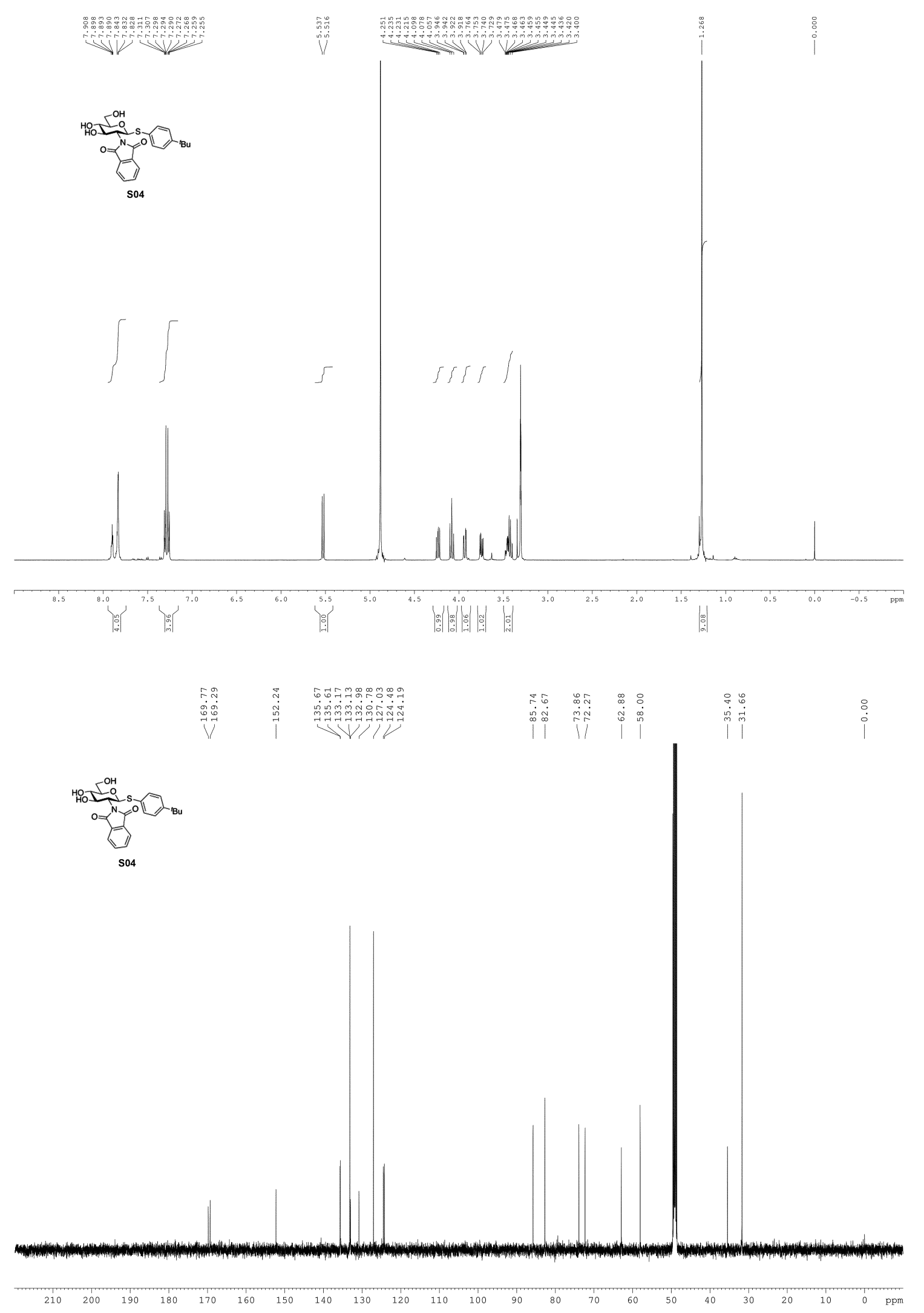



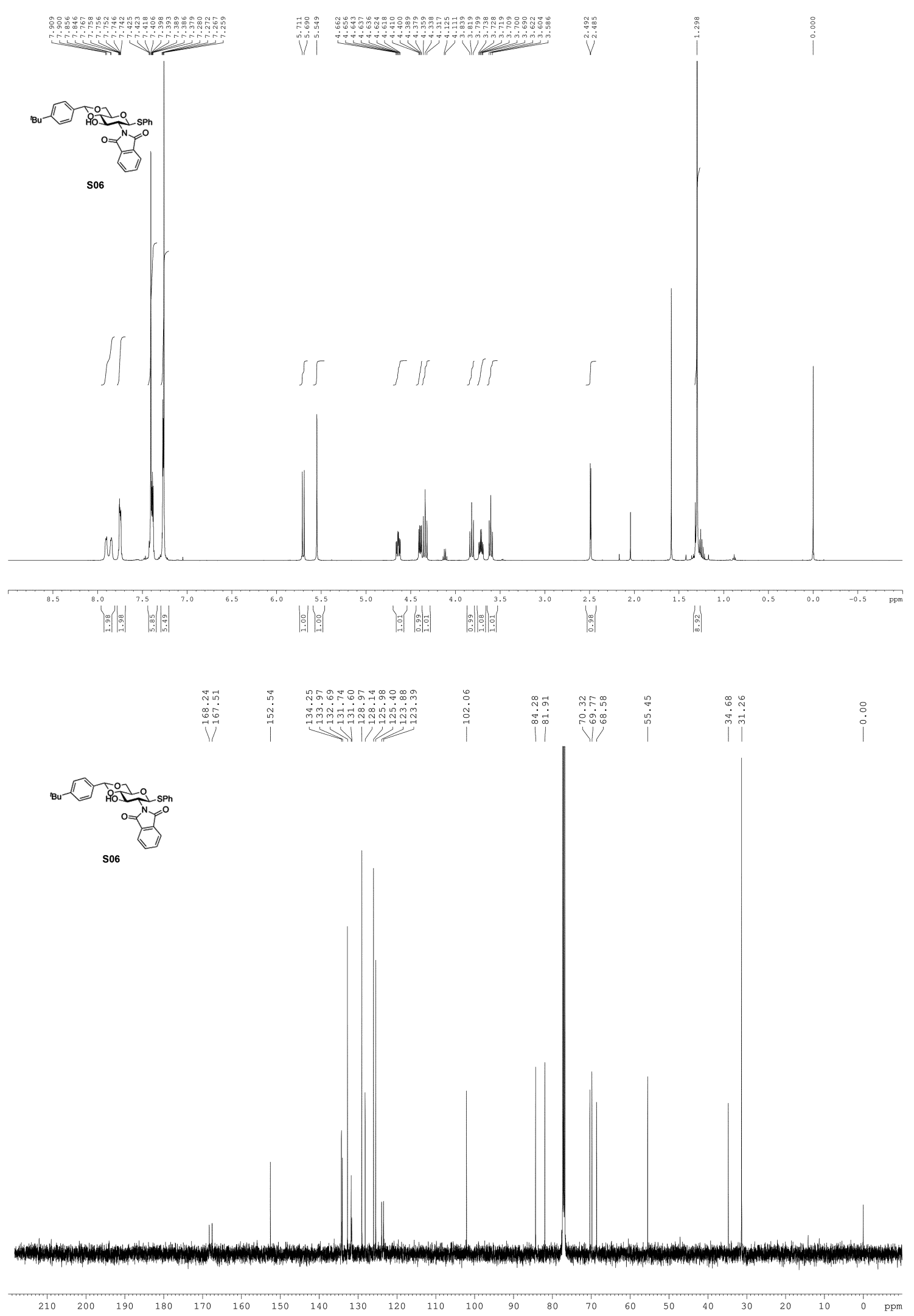


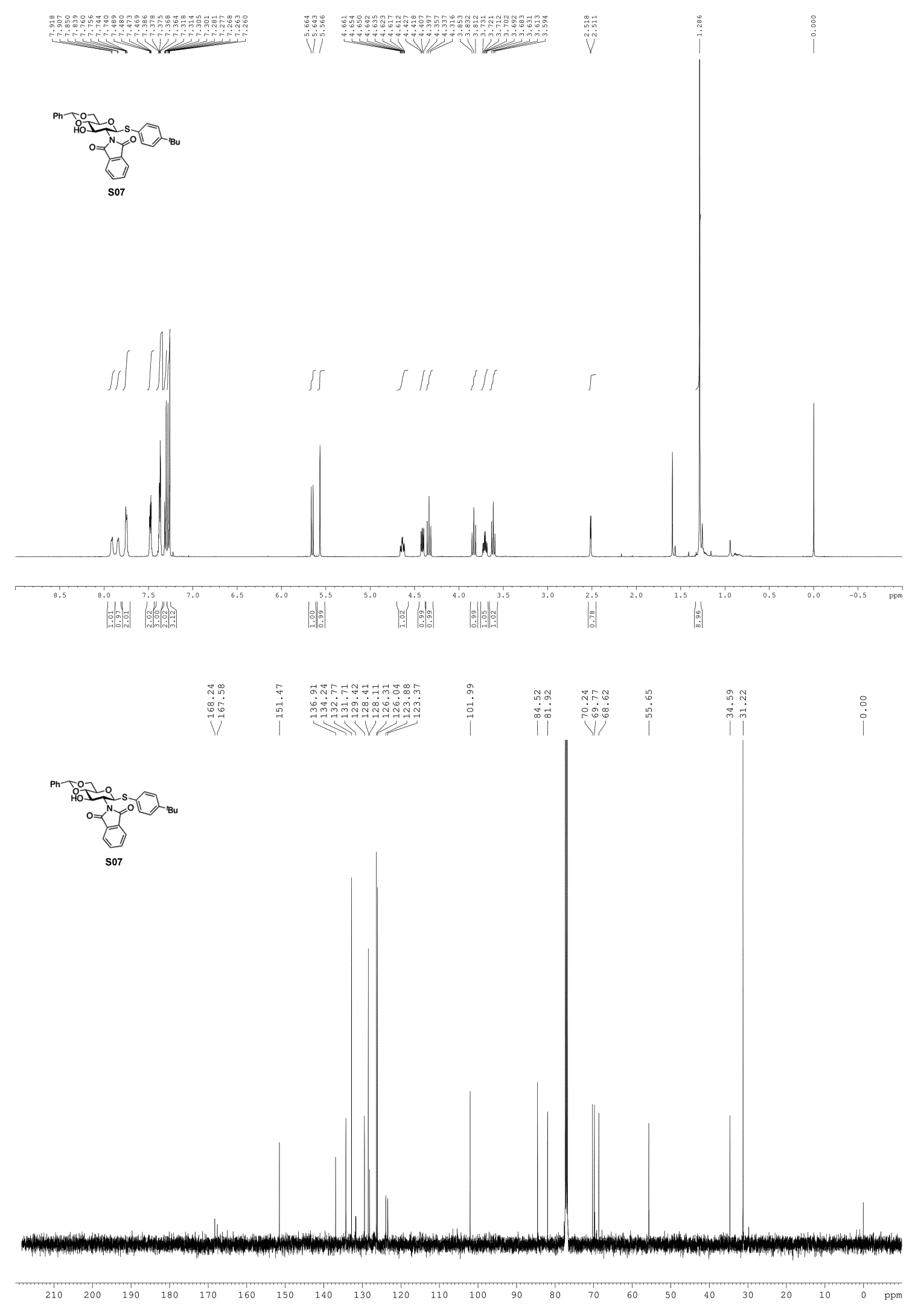




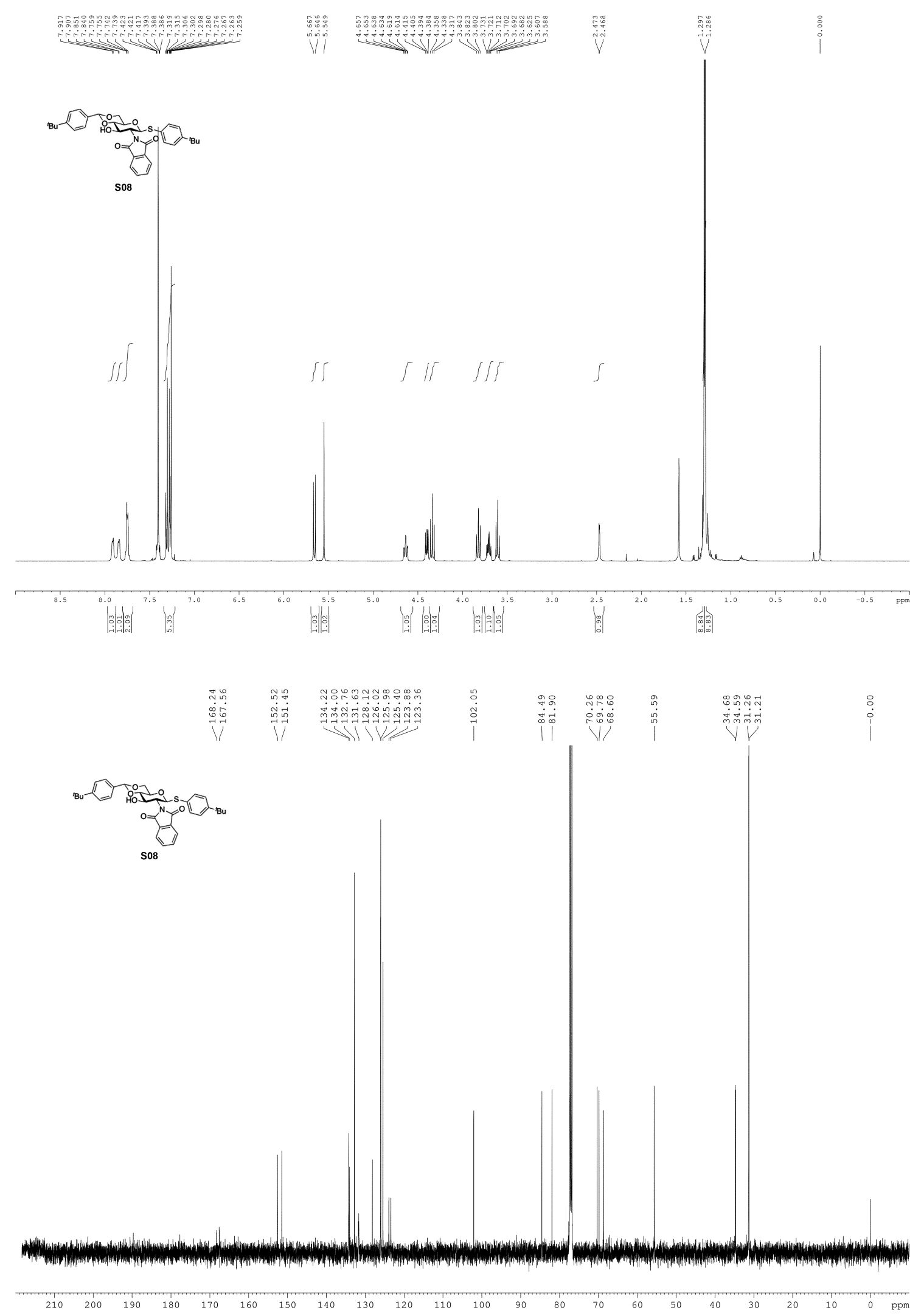




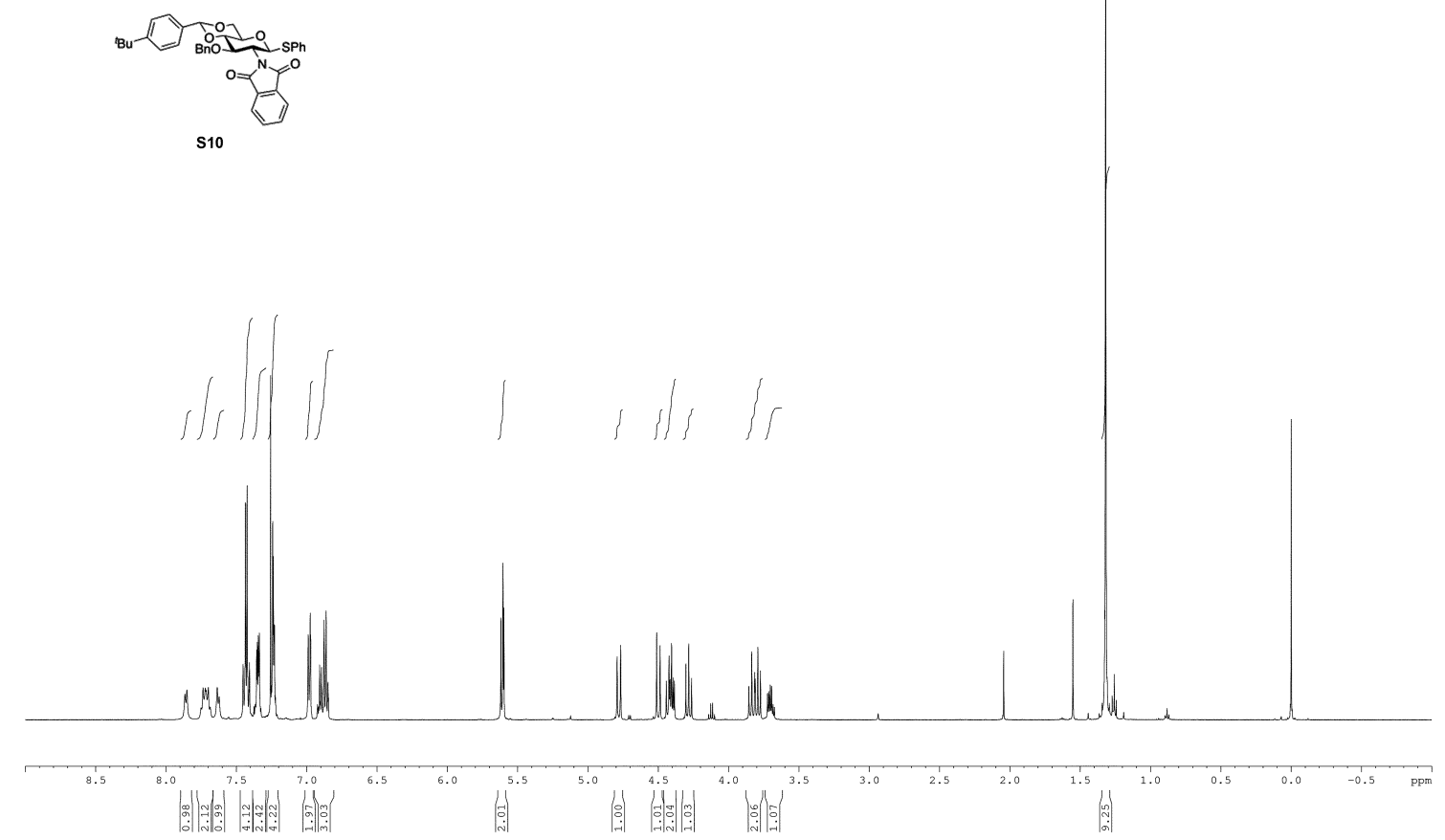

S10
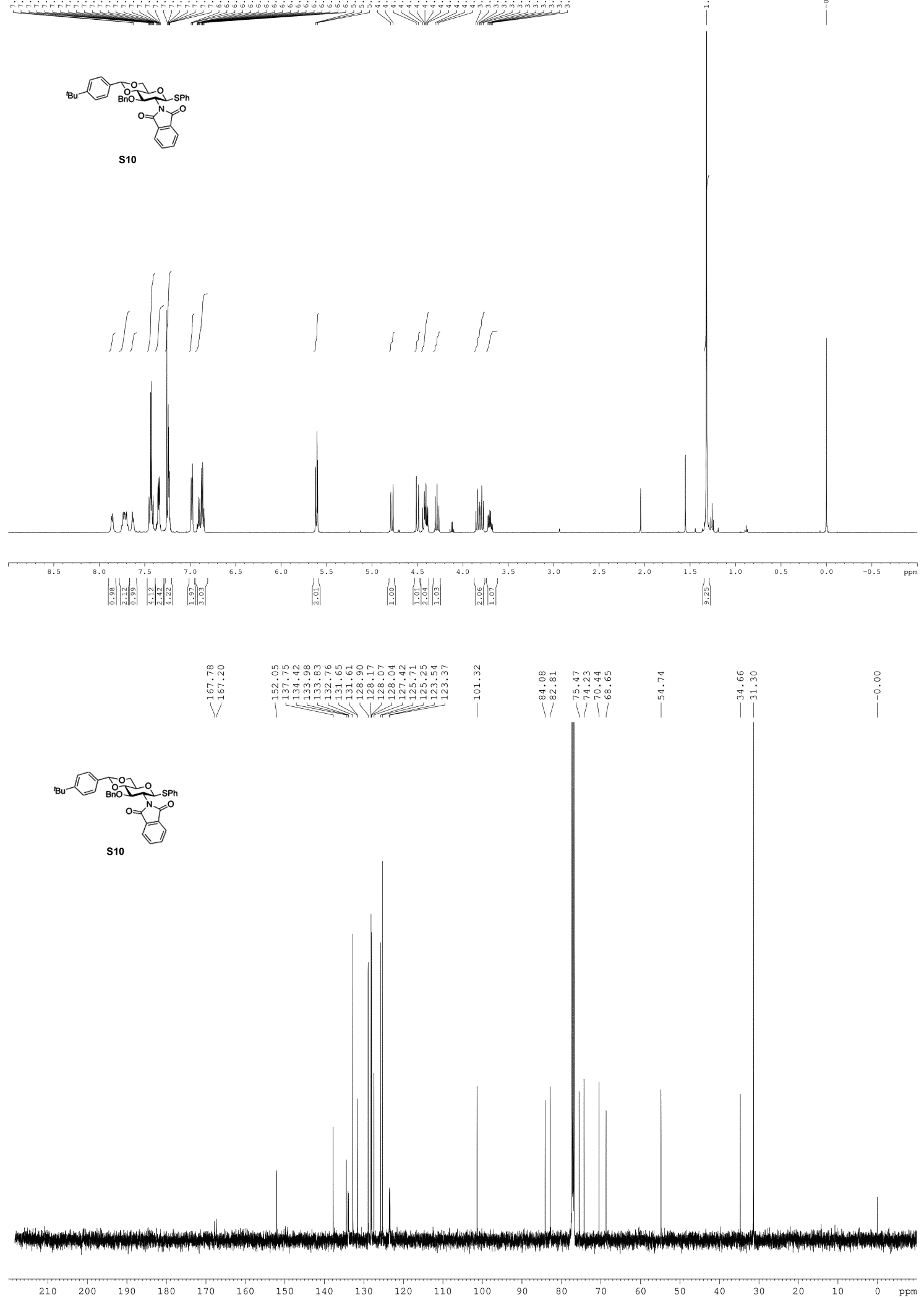

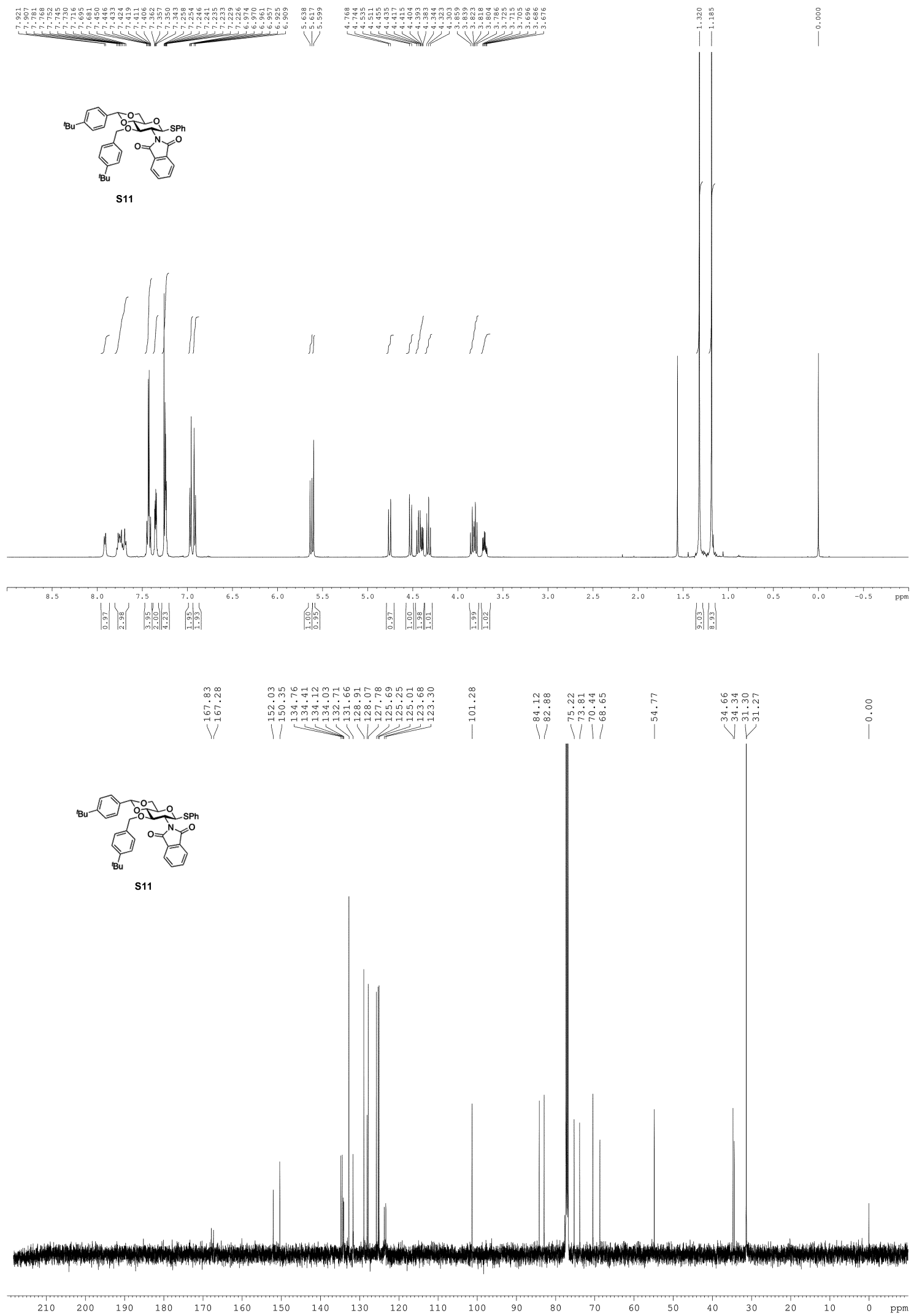


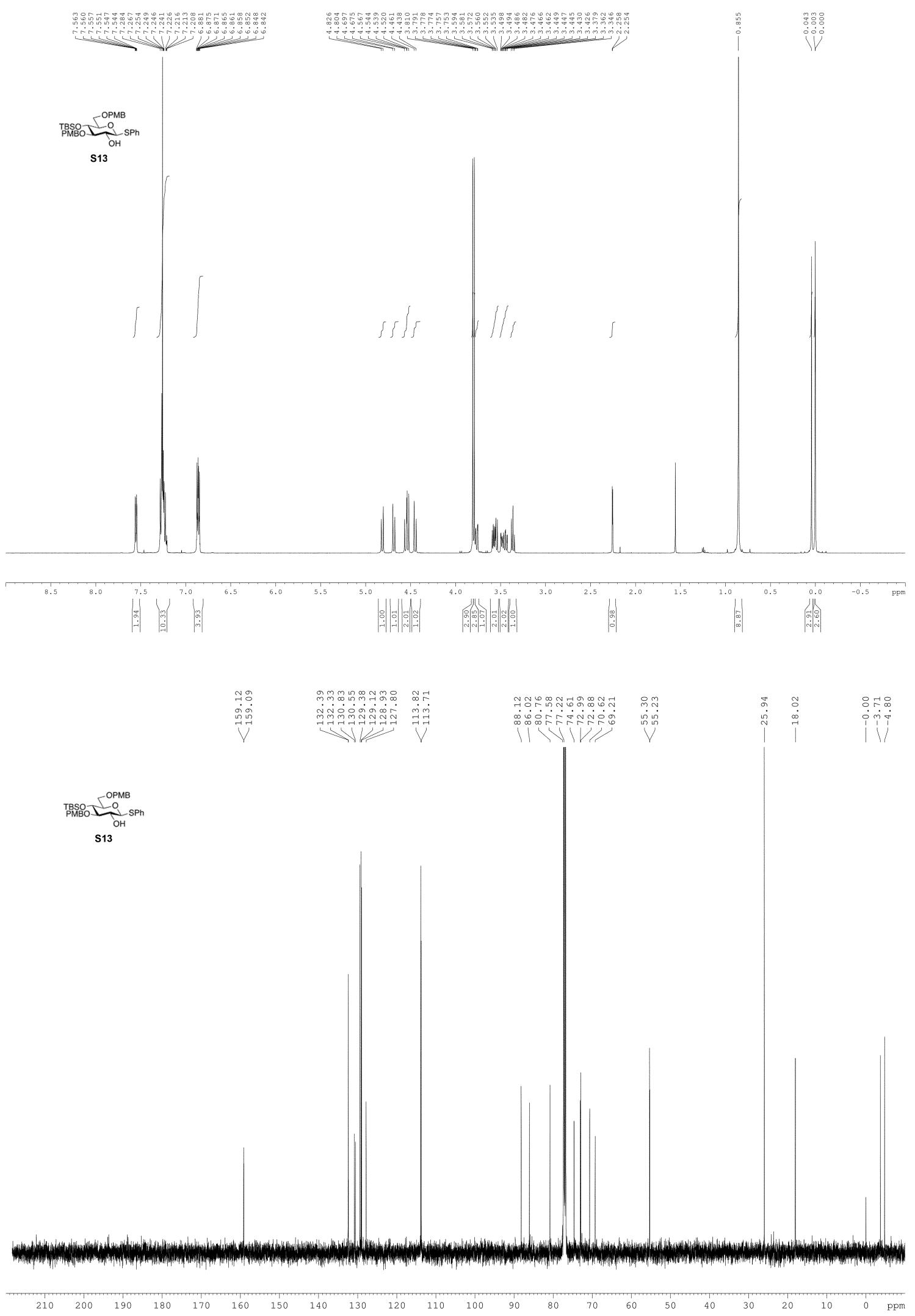



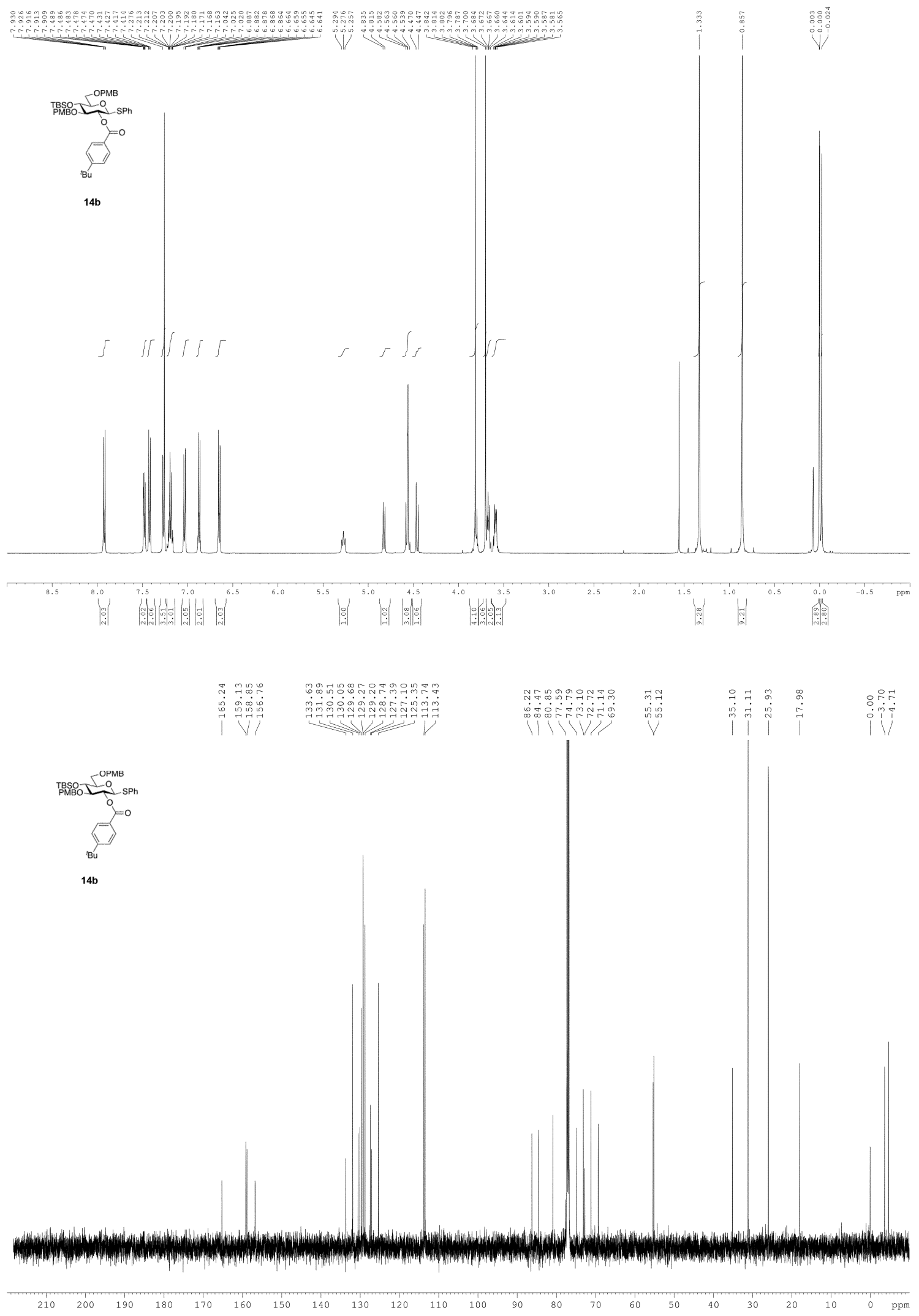


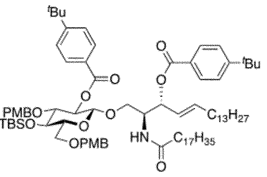

$16 \mathrm{~b}$

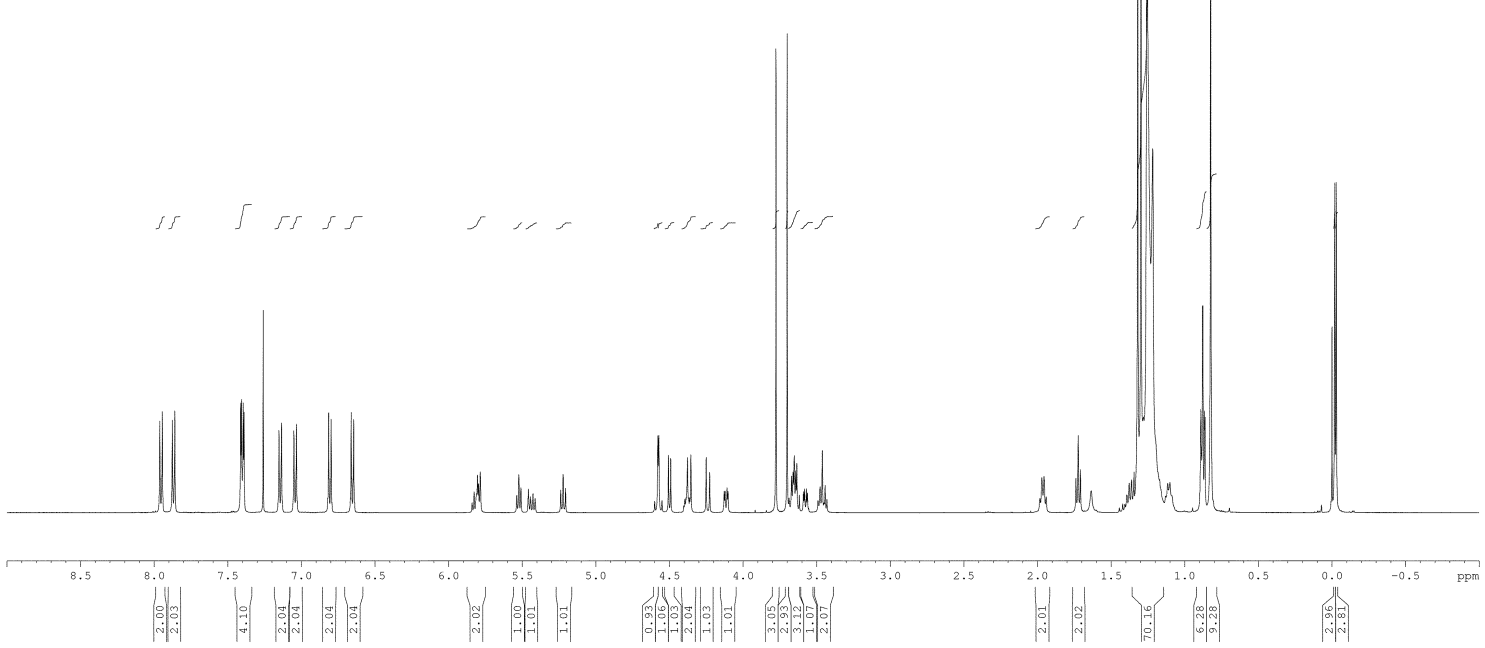

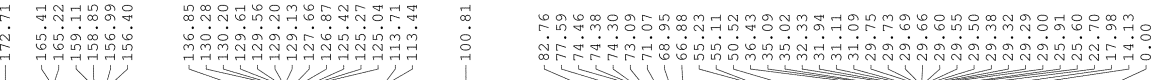

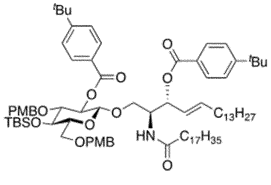

$16 \mathrm{~b}$

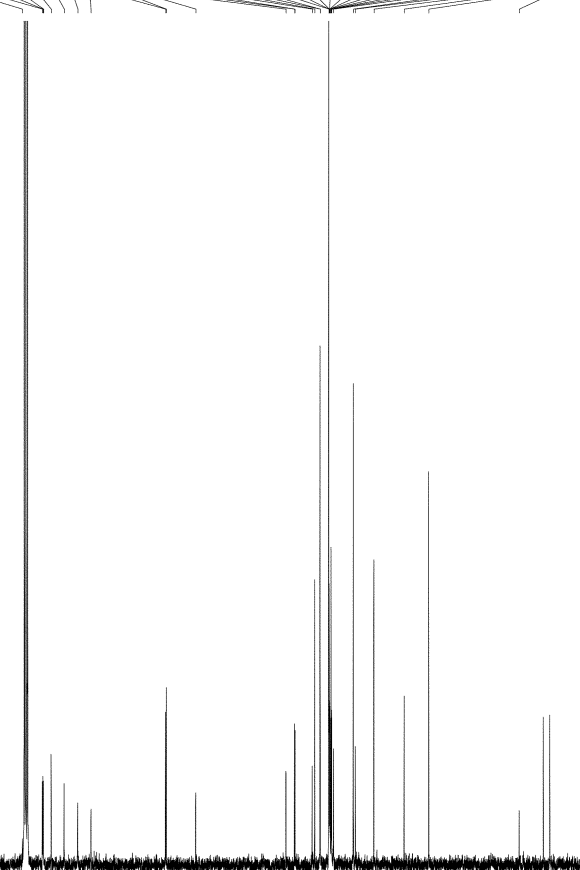

$\begin{array}{lllllllllllllllllllllll}210 & 200 & 190 & 180 & 170 & 160 & 150 & 140 & 130 & 120 & 110 & 100 & 90 & 80 & 70 & 60 & 50 & 40 & 30 & 20 & 10 & 0\end{array}$ 


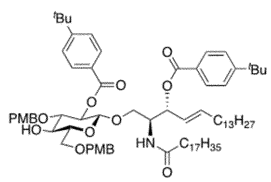

17b

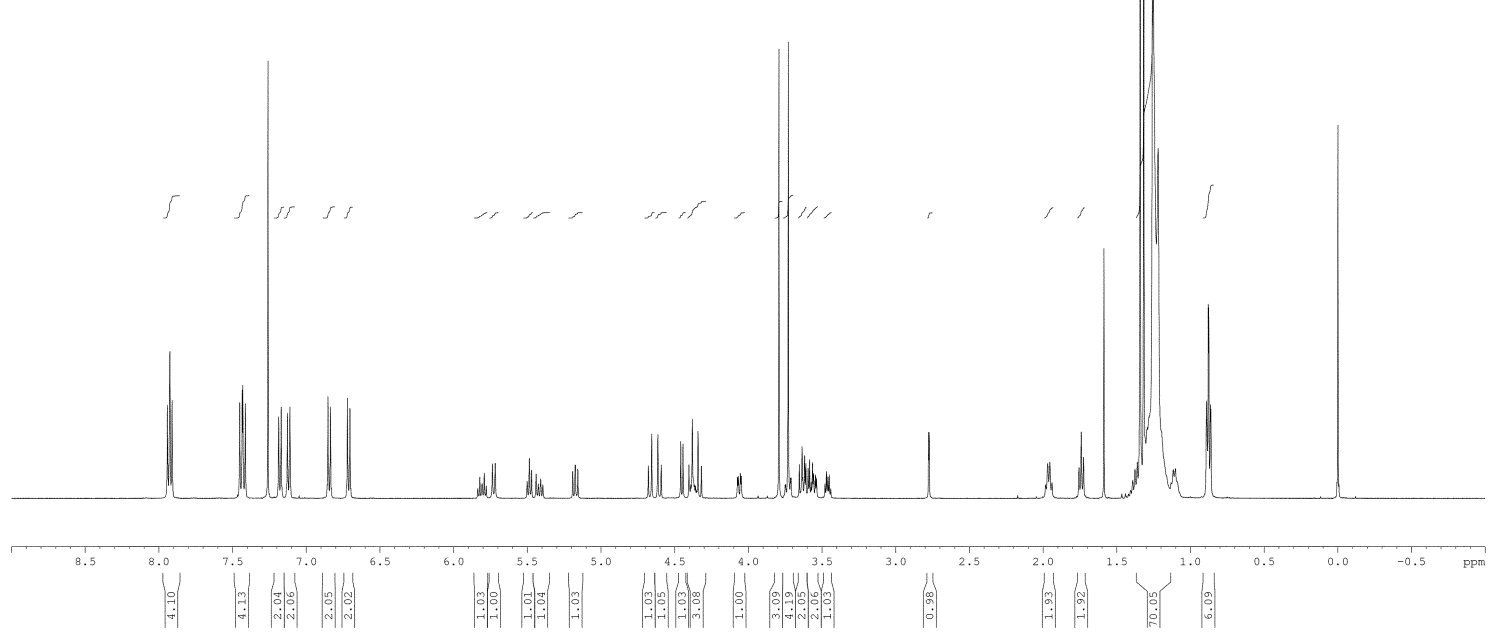

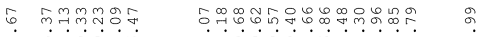

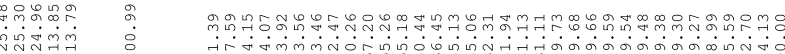

कि

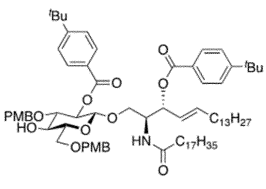

17b

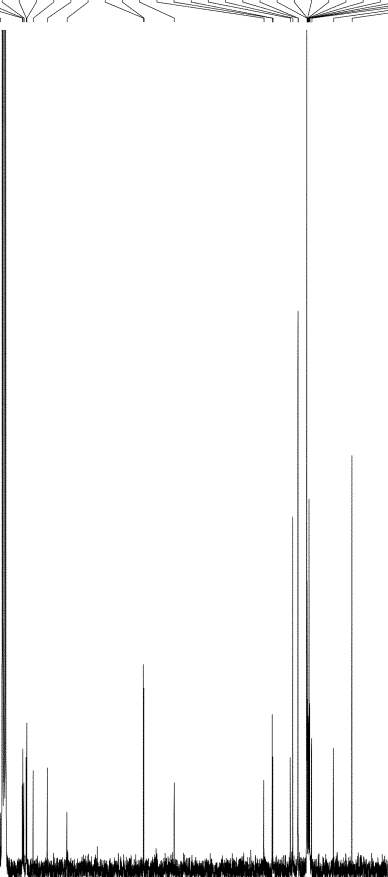




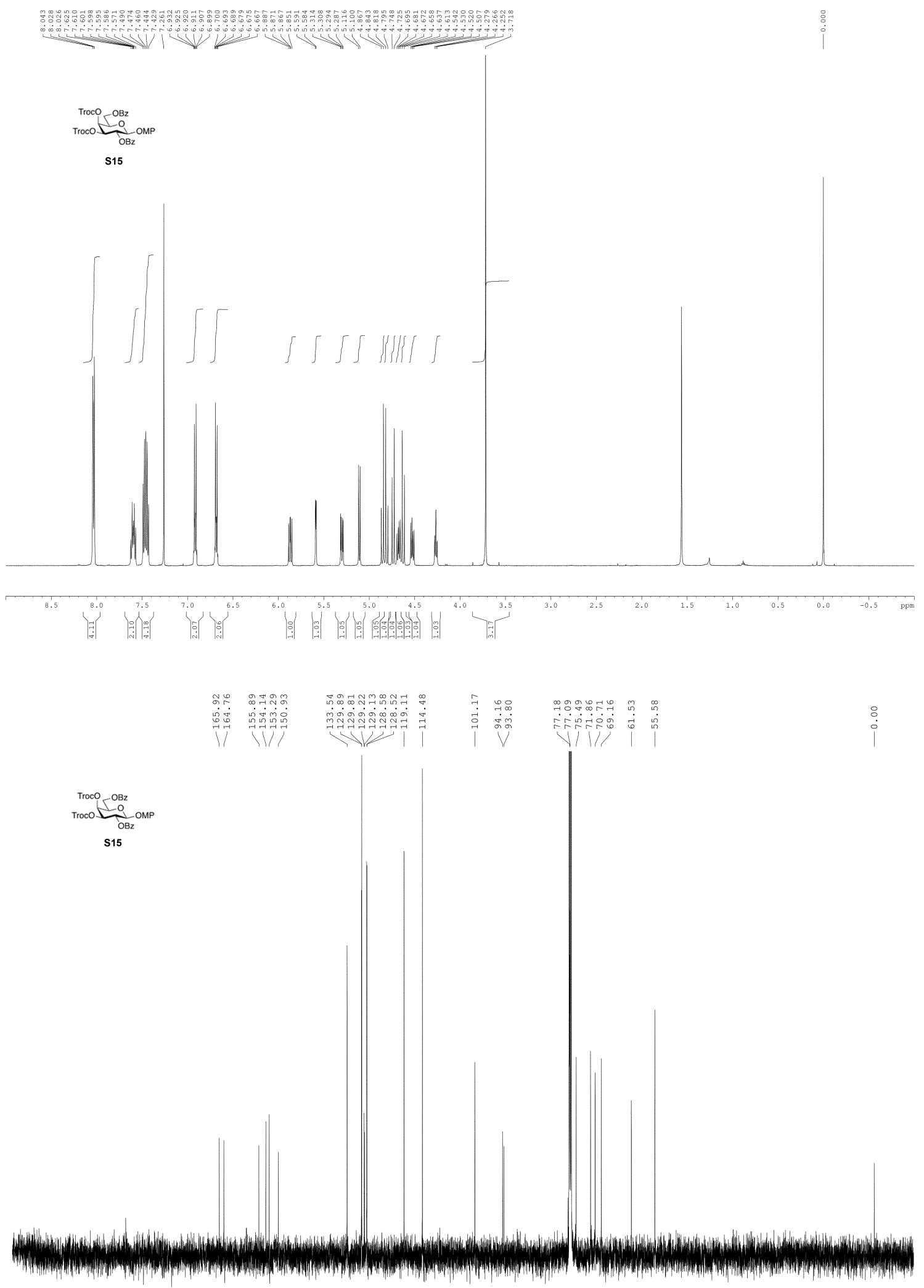

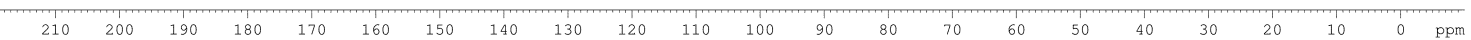



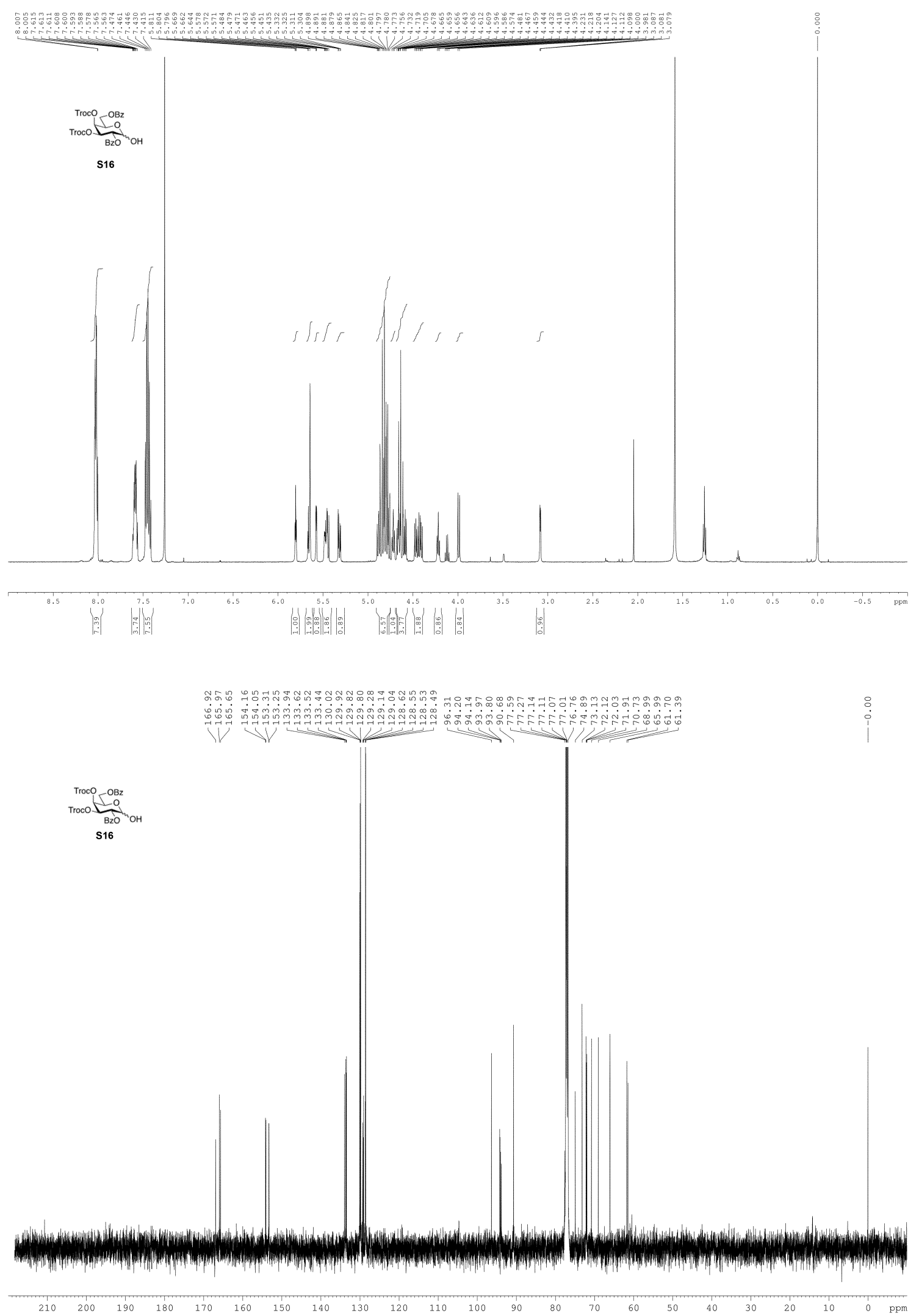

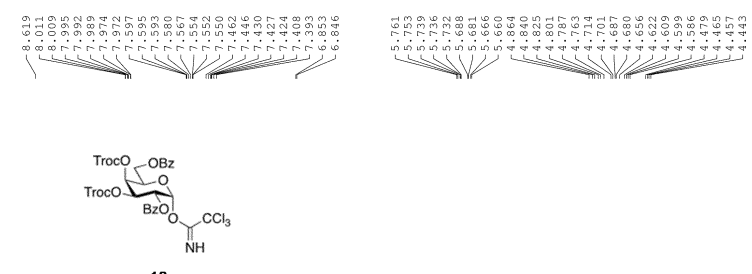

18

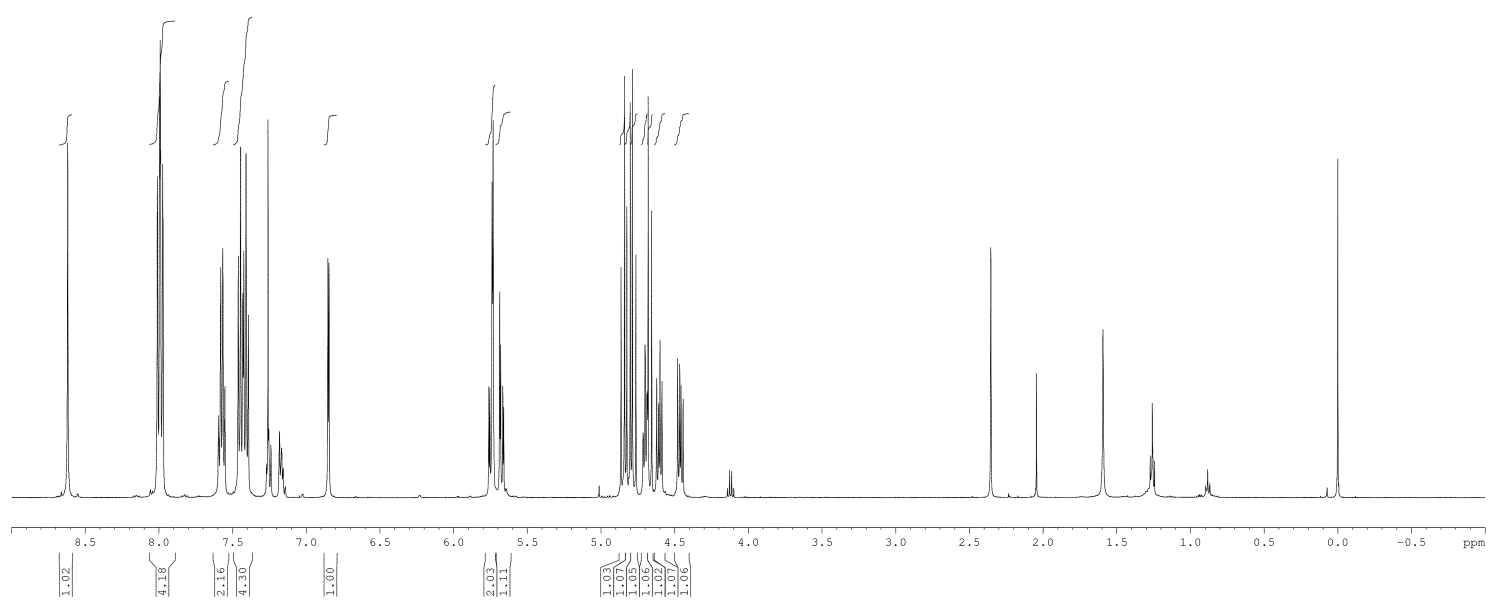

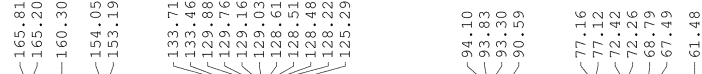

Troco $\underbrace{\mathrm{O} O \mathrm{O}_{\mathrm{NH}}^{\mathrm{O} z}}_{\mathrm{BzO}}$

18
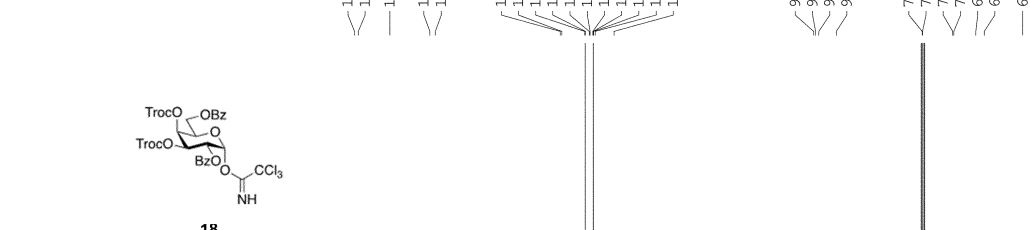

I I P

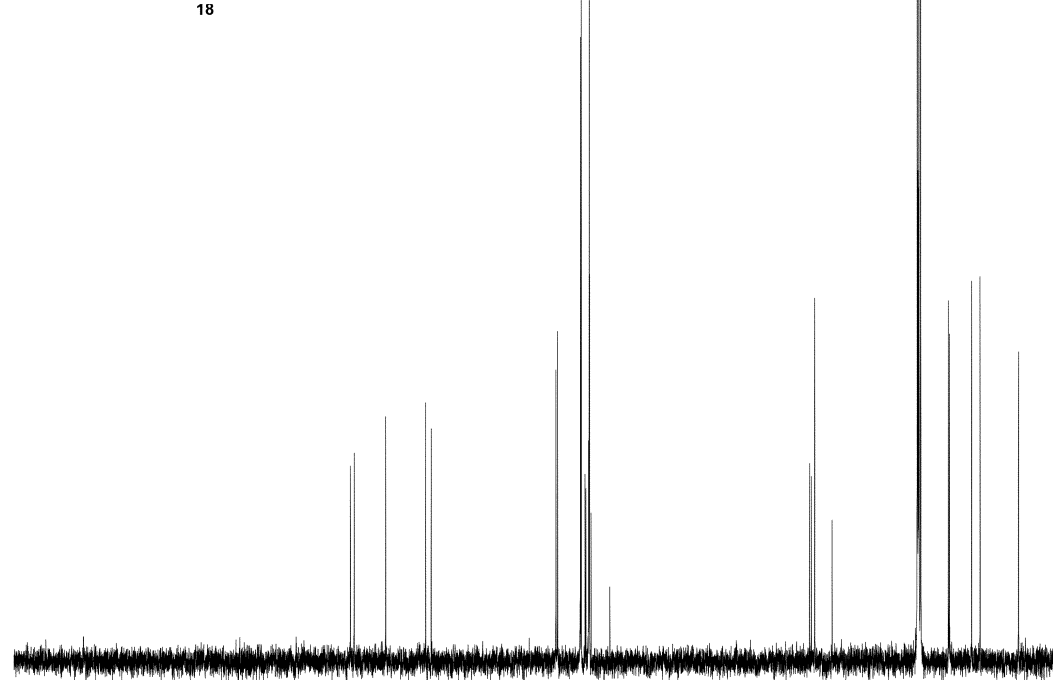

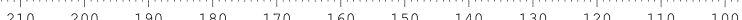

80

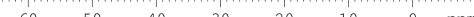




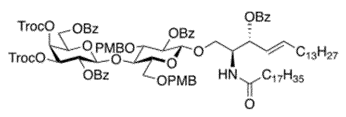

$19 a$

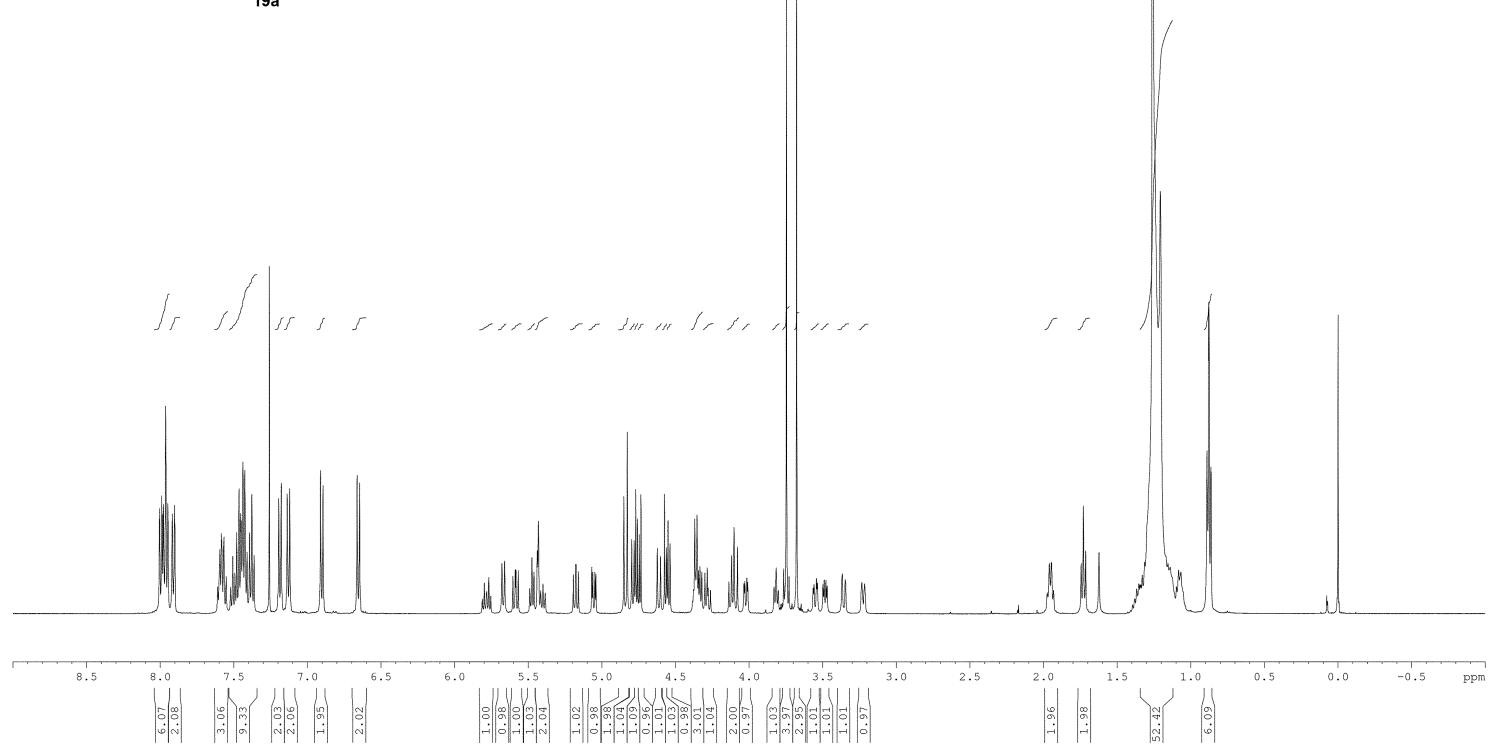

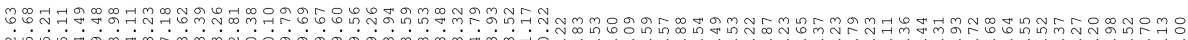

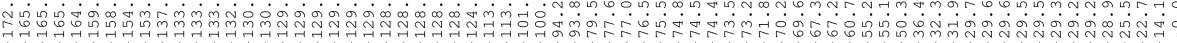

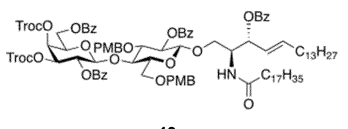

$19 a$

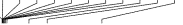

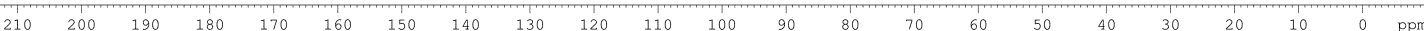




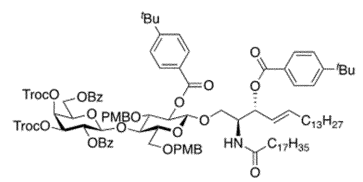

19b

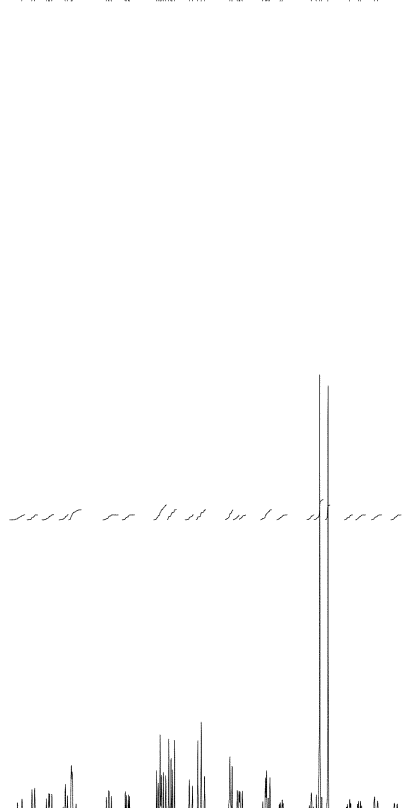

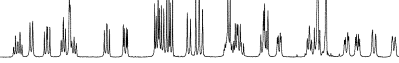
A

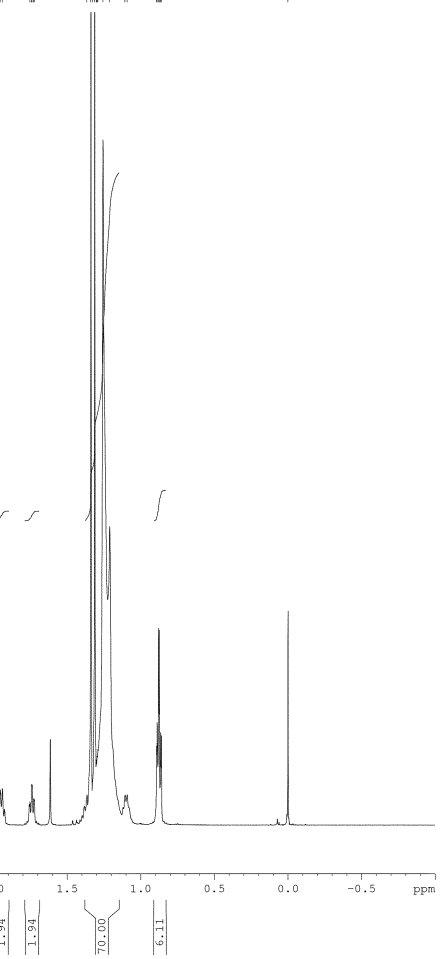

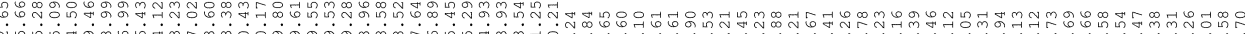

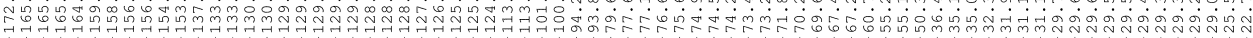

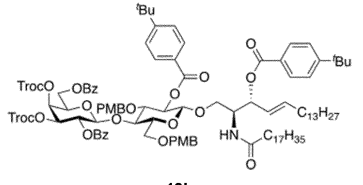

19b

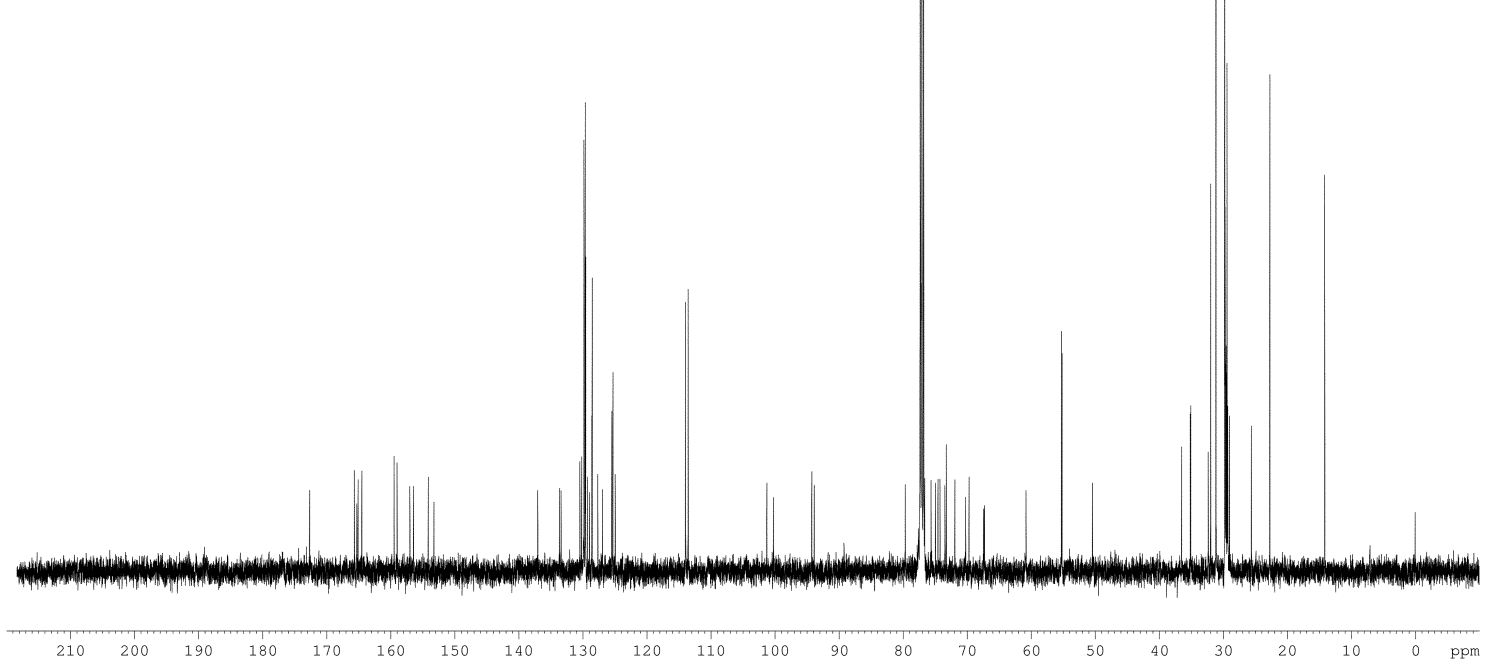




\section{References}

(S01) Imamura, A.; Ando, H.; Ishida, H.; Kiso, M. Org. Lett. 2005, 7, 4415-4418.

(S02) Chiara, J. L.; García, Á.; Cristóbal-Lumbroso, G. J. Org. Chem. 2005, 70, 4142-4151.

(S03) DeMeester, K. E.; Liang, H.; Jensen, M. R.; Jones, Z. S.; D’Ambrosio, E. A.; Scinto, S. L.; Zhou, J.; Grimes, C. L. J. Am. Chem. Soc. 2018, 140, 9458-9465.

(S04) Liu, R.; Wei, A. J. Carbohydr. Chem. 2012, 31, 384-419.

(S05) Nakashima, S.; Ando, H.; Saito, R.; Tamai, H.; Ishida, H.; Kiso, M. Chem. Asian J. 2012, 7, 10411051.

(S06) Gu, G.; Du, Y.; Pan, J. Carbohydr. Res. 2002, 337, 1313-1317. 